\title{
From Radical-Enhanced to Pure Thermal ALD of Aluminium and Gallium Nitrides
}




\section{From radical-enhanced to pure thermal ALD of gallium and aluminium nitrides}

Sourish Banerjee 
This dissertation has been approved by:

Supervisor:

prof. dr. D. J. Gravesteijn

Co-supervisor:

dr. A. Y. Kovalgin

This work is part of the project "Towards polycrystalline GaN/AlGaN devices in silicon technology" (no. 13145) funded by the Applied and Engineering Science (TTW) domain of the Netherlands Organization for Scientific Research (NWO). This work has been carried out at the

$\widehat{N W O} \mid \begin{aligned} & \text { Applied and } \\ & \text { Engineering Sciences }\end{aligned}$ MESA+ Institute for Nanotechnology, University of Twente.

Cover design and lay-out:

Back cover image:

Printed by:

ISBN:

DOI:
$\mathrm{PhD}$ candidate

The atypical dependence of the growth per cycle of thermal ALD

GaN layers on a precursor's partial pressure.

Gildeprint (Enschede, The Netherlands)

978-90-365-4825-0

$10.3990 / 1.9789036548250$

(C) 2018 Enschede, The Netherlands. All rights reserved. No parts of this thesis may be reproduced, stored in a retrieval system or transmitted in any form or by any means without permission of the author.

Alle rechten voorbehouden. Niets uit deze uitgave mag worden vermenigvuldigd, in enige vorm of op enige wijze, zonder voorafgaande schriftelijke toestemming van de auteur. 


\title{
FROM RADICAL-ENHANCED TO PURE THERMAL ALD OF GALLIUM AND ALUMINIUM NITRIDES
}

\section{DISSERTATION}

\author{
to obtain \\ the degree of doctor at the University of Twente, \\ on the authority of the rector magnificus, \\ prof. dr. T.T.M. Palstra, \\ on account of the decision of the Doctorate Board, \\ to be publicly defended \\ on Wednesday the $11^{\text {th }}$ of September 2019 at 16:45 hours \\ by

\section{Sourish Banerjee}

born on the $24^{\text {th }}$ of June 1988

in Kolkata, India 


\section{GRADUATION COMMITTEE:}

Chairman and Secretary: prof. dr. J. N. Kok (University of Twente)

Supervisor:

prof. dr. D. J. Gravesteijn (University of Twente)

Co-supervisor:

dr. A. Y. Kovalgin (University of Twente)

Special Expert:

dr. ir. J. W. Maes (ASM International N.V.)

Members:

prof. dr. ir. J. R. van Ommen (TU Delft)

prof. dr. ir. W. M. M. Kessels (TU Eindhoven)

prof. dr. C. Detavernier (University of Ghent)

dr. ir. R. J. E. Hueting (University of Twente)

prof. dr. ing. A. J. H. M. Rijnders (University of Twente) 
To my friends and colleagues at Twente 



\section{Contents}

1 Introduction............................................................ 1

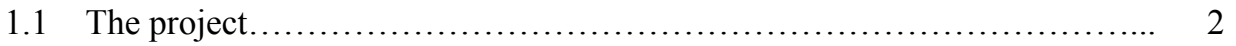

1.2 Group III-Nitride semiconductors: $\mathrm{GaN}$ and AlN....................... 2

1.2.1 From monocrystalline to polycrystalline........................... 5

1.3 Basic concepts of ALD ............................................... 7

1.3.1 Precursor choice for (Al)GaN ALD ............................ 11

1.3.2 ALD hardware ................................................. 12

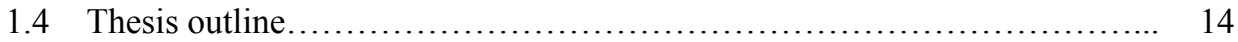

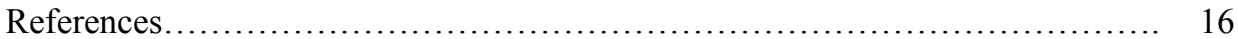

Appendix 1: Monitoring the wafer temperature with in-situ SE.................. 24

2 Plasma-enhanced ALD (PEALD) of polycrystalline AIN and GaN .... 25

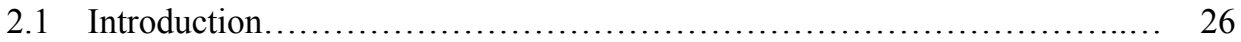

2.1.1 PEALD: A brief overview........................................ 27

2.2 PEALD of polycrystalline AlN layers................................. 28

2.2.1 Deposition conditions......................................... 28

2.2.2 ALD window determination................................... 28

2.2.3 In-situ and ex-situ characterization............................. 30

2.2.3.1 Spectroscopic ellipsometry and optical modelling........... 30

2.2.3.2 X-ray photoelectron spectroscopy ......................... 31

2.2.3.3 Rutherford backscattering spectroscopy................... 32

2.2.3.4 X-ray diffraction by $\theta-2 \theta$, Grazing incidence and $\omega$-scans... 33

2.2.4 Crystallinity optimization of PEALD AIN layers................... 34

2.2.4.1 Effect of plasma power................................ 34

2.2.4.2 Effect of in-situ substrate pre-treatment................... 35

2.2.4.3 Effect of rapid thermal annealing of in-situ deposited AlN seed layer............................................. 36

2.3 PEALD of polycrystalline GaN layers................................ 37

2.3.1 Ex-situ characterization........................................ 37 
2.3.1.1 X-ray photoelectron spectroscopy.................... 37

2.3.1.2 Fourier transform infrared spectroscopy.................. 39

2.3.1.3 Scanning and Transmission electron microscopy........... 39

2.3.1.4 Grazing incidence X-ray diffraction.................... 41

2.3.2 Effect of plasma composition on the polycrystallinity of GaN....... 41

2.3.3 Comparing the effect of plasma composition on the polycrystallinity of AlN

2.4 Conclusions......................................................... 45

References........................................................ 46

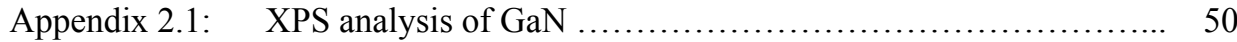

Appendix 2.2: $\quad$ Low energy ion scattering (LEIS)....................... 51

Appendix 2.3: Spectroscopic ellipsometry of PEALD GaN............... 52

Appendix 2.4: Optical emission spectroscopy (OES)..................... 53

\section{Towards ALD of AIN and GaN using radicals generated by a}

hot-wire ........................................................... 59

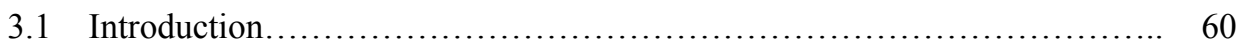

3.1.1 The hot-wire hardware .................................. 60

3.2 Tests for radical generation....................................... 61

3.2.1 Catalytic dissociation of $\mathrm{H}_{2}$ : Generation of at-H radicals............. 61

3.2.1.1 In-situ monitoring Te etching by at-H radicals............. 62

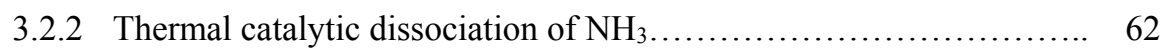

3.2.2.1 Generation of at-H radicals.......................... 62

3.2.2.2 Generation of $\mathrm{NH}_{\mathrm{x}}(x=0-2)$ radicals................. 63

3.3 HWALD of AlN and oxygen contamination.......................... 65

3.3.1 Deposition outside the L-O-S position........................... 65

3.3.2 Deposition in the L-O-S position ............................. 66

3.3.2.1 Effect of filament temperature...................... 67

3.3.2.2 Change in crystallinity with position on the wafer.......... 68

3.3.2.3 Effect of reactor pressure.......................... 69

3.3.2.4 Effect of removing sources of oxidants.................. 70

3.3.2.5 Analysis of the chemical phases of the ring............... 71 
3.4 Towards HWALD of GaN: Formation of Ga droplets................. 72

3.4.1 Deposition outside the L-O-S position........................ 72

3.4.2 Deposition in the L-O-S position ............................... 73

3.5 The action of at-H on chemisorbed TMA and TMG.................... 74

3.6 Conclusions...................................................... 76

References............................................................ 77

Appendix 3.1: Dependence of the refractive index on oxynitride composition.. 79

Appendix 3.2: $\quad$ Additional characterization of the reactor by at-H............. 80

4 Thermal ALD of composite $\mathbf{G a N}-\mathrm{C}-\mathrm{Ga}$ ('GaCN') layers............. 83

4.1 Introduction...................................................... 84

$4.2 \mathrm{GaCN}$ deposition and characterization.............................. 85

4.2.1 Layer cross section and morphology.......................... 85

4.2.2 Chemical bonding and phase-segregation analyses................ 86

4.2.2.1 FTIR analysis..................................... 86

4.2.2.2 XPS analysis..................................... 87

4.2.2.3 XRD analysis..................................... 90

4.2.2.4 HR-TEM and EF-TEM analyses....................... 91

4.2.3 GaCN optical constants determined by SE...................... 94

4.2.3.1 Origin of the optical constants' variation.................. 96

$4.3 \mathrm{GaCN}$ growth kinetics.......................................... 98

4.3.1 Formation of $\mathrm{GaN}$ clusters..................................... 98

4.3.2 Formation of Ga- and C-containing inclusions................... 99

4.4 Conclusions.................................................. 102

References......................................................... 103

Appendix 4: Exploiting the high refractive index of $\mathrm{GaCN}$ in Distributed

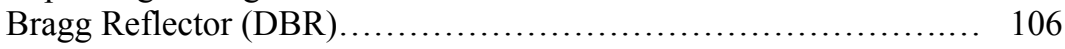

5 Thermal ALD of polycrystalline GaN ............................ 111

$5.1 \quad$ Introduction....................................................... 112

5.2 Review of previous GaN ALD reports............................ 112

5.2.1 Novelty of the proposed GaN ALD process..................... 113 
5.3 Surface reactions leading to GaN ALD.............................. 114

5.3.1 Reported mechanism of thermal AIN ALD ...................... 114

5.3.2 Proposed mechanism of thermal GaN ALD...................... 114

5.4 GaN MOCVD reactions from $\mathrm{TMG}$ and $\mathrm{NH}_{3}$ precursors................ 115

5.4.1 The adduct pathway in more detail.............................. 117

5.5 Modelling the GaN ALD kinetics.................................... 118

5.5.1 TMG: $\mathrm{NH}_{3}$ surface adduct formation........................... 118

5.5.2 $\mathrm{Ga}-\mathrm{NH}_{2}-\mathrm{Ga}$ linkage formation................................................ 119

5.6 The GaN ALD window......................................... 120

5.6.1 Variation of GPC with $t_{\mathrm{NH} 3}, P_{\mathrm{NH} 3}$ and T........................ 120

5.6.2 Anomalous variation of GPC with post- $\mathrm{NH}_{3}$ purge time............ 121

5.7 Growth analysis by in-situ SE.................................... 122

5.7.1 Effect of $t_{\mathrm{NH} 3}$ on step height............................... 123

5.7.2 Effect of $P_{\mathrm{NH} 3}$ on step height............................... 123

5.7.3 Effect of post- $\mathrm{NH}_{3}$ purge duration on step height................. 125

5.8 Layer characterization.............................................. 125

5.8.1 HR-SEM analysis............................................ 125

5.8.2 GIXRD and HR-TEM analyses............................ 125

5.8 .3 FTIR analysis.............................................. 127

5.8 .4 XPS analysis................................................ 127

5.8.4.1 Stoichiometry control of the layers..................... 129

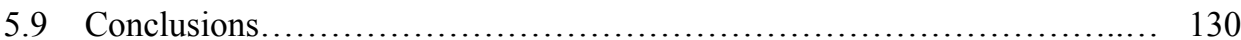

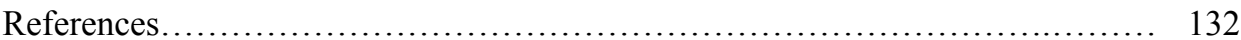

Appendix 5: Mathcad script for simulating the $\mathrm{TMG}: \mathrm{NH}_{3}$ surface adduct and GaN formation............................................... 136

6 Inherent substrate-selective thermal ALD of GaN .................. 145

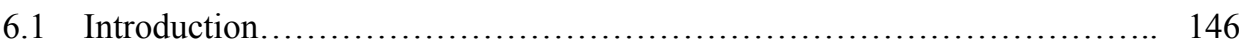

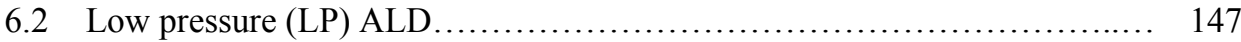

6.2.1 GaN growth enhancement by ALD AlN buffer layer............... 147

6.2.2 GaN growth enhancement by AlN monolayers deposited within super-cycles... 


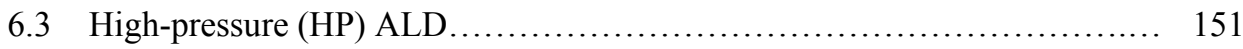

6.3.1 Dependence of incubation time on surface termination............... 151

6.3.2 Control experiments showing the role of $-\mathrm{NH}_{2}$ terminations and the effect of plasma................................................... 152

6.4 Investigation into the growth on Si and AIN substrates by in-situ SE......... 153

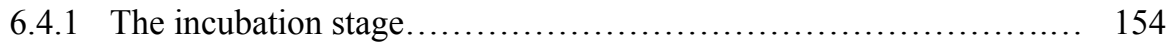

6.4.2 The stage of increasing step height................................ 154

6.4.3 The stage of stable step height................................... 154

6.5 Towards thermal ASALD of GaN ...................................... 155

6.5.1 ALD on patterned $\mathrm{AlN} / \mathrm{SiO}_{2}$ substrate ......................... 156

6.5.2 ALD on patterned $\mathrm{Si}_{3} \mathrm{~N}_{4} / \mathrm{SiO}_{2} / \mathrm{Si}$ substrate ...................... 158

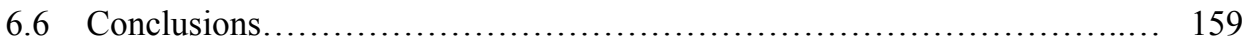

References........................................................... 160

Appendix 6.1: $\quad$ Wet etching of ALD (Al)GaN layers........................... 161

Appendix 6.2: $\quad$ Scanning electron microscope images of GaN ALD performed on the ex-situ patterned $\mathrm{AlN} / \mathrm{SiO}_{2}$ substrate................... 162

7 Thermal ALD of AIN and estimation of layer coalescence.............. 163

7.1 Introduction.......................................................... 164

7.2 Why ALD does not necessarily imply a coalesced layer.................... 164

7.2.1 Types of initial growth in ALD processes.......................... 164

7.2.2 Substrate-inhibited ALD.......................................... 166

7.2.3 Tests for the substrate-inhibited ALD of AlN on PtSi: A brief

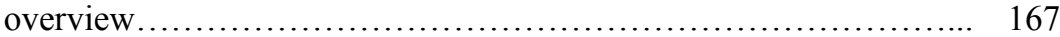

7.2.3.1 With M-I-M structures................................... 167

7.2.3.2 With in-situ SE........................................ 167

7.2.3.3 With electron microscopy .............................. 167

7.3 Thermal ALD of AlN and material properties............................. 168

7.4 Test for AlN coalescence (with M-I-M structures) ........................... 169

7.4.1 M-I-M fabrication.............................................. 169

7.4.2 M-I-M without AlN layer....................................... 171

7.4.3 M-I-M with AlN layers of various thicknesses.................... 171

7.4.3.1 AlN in the pre-coalesced state.......................... 171 
7.4.3.2 AlN in the coalesced state............................ 172

7.4.3.3 AlN in the transition state.............................. 173

7.5 Test for AlN coalescence (with in-situ SE) .......................... 174

7.6 Pre-coalesced AIN clusters observed with electron microscopy.............. 175

7.7 Conclusions..................................................... 178

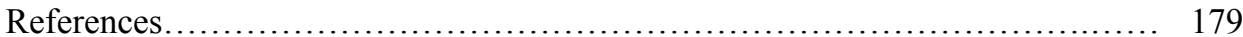

Appendix 7: $\quad$ Schematics and equivalent electrical circuits of the M-I-M test structure before and after coalescence..................... 181

8 Conclusions and future recommendations......................... 183

8.1 Conclusions......................................................... 184

8.2 Future recommendations........................................... 186

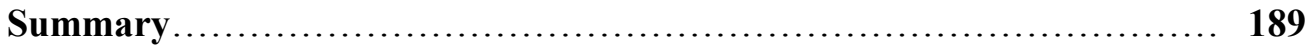

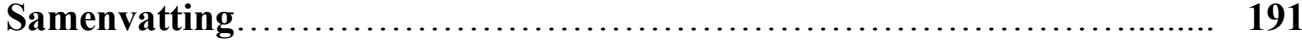

Scientific contributions........................................... 195

Acknowledgements............................................ 199 




\section{Introduction}




\subsection{The project}

The project 'Towards polycrystalline GaN/AIGaN devices in silicon technology' is funded by the Nederlandse Organisatie voor Wetenschappelijk Onderzoek (NWO), number 13145. The project aimed at studying the deposition and the potential applications of thin films (sub-100 nm) of polycrystalline Group III-Nitride semiconductors (specifically, gallium nitride $(\mathrm{GaN})$ and aluminium nitride $(\mathrm{AlN})$ ), by a process compatible with siliconbased integrated circuits (IC) technology. Keeping in mind the potential application of the results for the current and future micro- and opto-electronics industries, Atomic Layer Deposition (ALD) technique was used chosen to prepare these films, using standard industrial precursors.

Specific research questions were targeted in the project. Some of them include: can ALD be a viable technique for preparing GaN and AlN thin films from the chosen set of precursors? What kind of activation techniques are necessary? Can the novel, in-housedeveloped hot-wire-assisted ALD technique be applicable for these films? Can GaN thin films be prepared using (hitherto-unknown approach of) purely thermal activation? How do the film properties vary with the deposition parameters? Can the insights obtained from investigating the growth mechanism of these layers be used for (the industrially-relevant topic of) area-selective ALD? Et cetera.

To address such questions, the thesis broadly comprised:

i. Development of ALD recipes for polycrystalline GaN and AlN thin films.

ii. Exploring plasma-enhanced, hot-wire-assisted, and thermal ALD techniques for the above.

iii. Analysing the thin film properties by in-situ and ex-situ techniques.

Whereas this thesis focusses on the deposition and material characterization of such films, a related work (under the same project) has been undertaken to explore their electronic properties. The results obtained from the project are expected to advance the state-of-the-art of polycrystalline III-Nitride ALD technology. Sections 1.2 and $\mathbf{1 . 3}$ will gradually motivate the relevance of the project.

\subsection{Group III-Nitride semiconductors: GaN and AIN}

GaN and AlN belong to the group III-Nitride semiconductors that are extensively used for specific electronic and opto-electronic applications, and also in a number of other 
applications ranging from microelectromechanical systems (MEMS) to medical devices ${ }^{1-4 *}$. These applications rely on their excellent material properties in the monocrystalline form, such as direct and wide bandgap (6.2 eV for AlN, $3.4 \mathrm{eV}$ for $\mathrm{GaN})$, high breakdown field (5 and 3.6 MV cm $\mathrm{MV}^{-1}$, respectively), and high carrier mobility $\left(1500 \mathrm{~cm}^{2} \mathrm{~V}^{-1} \mathrm{~s}^{-1}\right.$ for electrons in $\mathrm{GaN})^{5}$. In addition, GaN, $\mathrm{AlN}$ and their alloy $\mathrm{Al}_{\mathrm{x}} \mathrm{Ga}_{1-\mathrm{x}} \mathrm{N}(x=0-1)$ exhibit polarity and piezoelectric properties ${ }^{6}$. Utilizing these properties, stacking $\mathrm{Al}_{\mathrm{x}} \mathrm{Ga}_{1-\mathrm{x}} \mathrm{N}$ on top of $\mathrm{GaN}$ or AlN enables a high-mobility 2-dimensional electron gas (2DEG) channel at the AlGaN/GaN interface due to band-offsets and polarization effects at the interface ${ }^{6}$. This is utilized in highspeed transistors, referred to as high electron mobility transistors or HEMTs ${ }^{7}$. Besides, they are intensively utilized in light emitting diodes (LEDs) and high frequency (RF) transistors ${ }^{8-}$ 9 .

Whereas GaN, AlN and $\mathrm{Al}_{\mathrm{x}} \mathrm{Ga}_{1-\mathrm{x}} \mathrm{N}$ (further collectively referred to as (Al)GaN)) dominate the field of opto-electronics, silicon ( $\mathrm{Si}$ ) remains the de facto semiconductor for a big share of the consumer-grade microelectronics market, such as in microprocessors and memories. This is because of the relatively low price and high integration level of $\mathrm{Si}$, as a consequence of the high degree of development of the Si technology, which is not the case yet for (Al)GaN technology. Some of the above-mentioned material properties of (Al)GaN (such as the breakdown field) are in fact superior to silicon. Therefore, the question arises: why has $\mathrm{Si}$ managed to achieve an exceptional level of industrial acceptance for microelectronics, whereas $(\mathrm{Al}) \mathrm{GaN}$ has not?

The developments in consumer electronics has been propelled by semiconductors such as germanium $(\mathrm{Ge})$ and $\mathrm{Si}^{10}$. Looking back at history, following the invention of Gebased transistors, it was soon realized that Si-based transistors performed better (due to, for example, lower reverse currents ${ }^{11}$ ). The superior insulating character of silicon dioxide $\left(\mathrm{SiO}_{2}\right)$ also became known ${ }^{10}$. Exploiting the passivation effects of $\mathrm{SiO}_{2}$, the first planar transistor was proposed in $1960^{10}$. The planar fabrication concept eventually led to integrated circuits (IC), ushering in the so-called information age. In $\mathrm{ICs}, \mathrm{SiO}_{2}$ served as the passivation layer, allowing metals like aluminium (Al) to be directly deposited on it, serving as the interconnect ${ }^{12}$. Thereafter, the dielectric properties of $\mathrm{SiO}_{2}$ (and aided by the achievement of an extremely high quality $\mathrm{Si} / \mathrm{SiO}_{2}$ interface) was utilized to realize the metal oxide field effect transistor (MOSFET) and subsequently, the complementary MOSFET (CMOS) circuits $^{13-15}$. In MOSFETs, Si (in the polycrystalline form) was preferred over metals, as the gate-material, as it could be used to make self-aligned devices ${ }^{16}$. Due to the easy availability of $\mathrm{Si}$, all these developments significantly advanced the Si technology, therefore aiding in the rapid miniaturization of transistors and increasing the number of transistors in ICs, in

\footnotetext{
* Due to the scope of this thesis, the subsequent discussion is based solely on the microelectronic and opto-electronic applications of these materials.
} 
accordance with the so-called Moore's law ${ }^{17}$. In comparison, even though the semiconducting and opto-electronic properties of the group III-V materials (e.g., GaP, GaAs) were discovered already in the $1950 \mathrm{~s}^{18}\left((\mathrm{Al}) \mathrm{GaN}\right.$ came much later $\left.{ }^{19}\right)$, their applications remained majorly confined in the opto-electronic domain.

A second reason behind the limitation of the commercialization of $(\mathrm{Al}) \mathrm{GaN}$ lies in the cost. The attractive material properties of $(\mathrm{Al}) \mathrm{GaN}$ are valid only when they are monocrystalline. Whereas the mass-production of monocrystalline Si has been achieved using the Czochralski method ${ }^{20}$, that of $(\mathrm{Al}) \mathrm{GaN}$ has not. Their monocrystalline preparation, e.g., by the Czochralski approach, is frequently encountered by phase segregation into the respective elements ${ }^{21}$. Moreover, the melting temperatures of (Al)GaN are between $2200-$ $2500{ }^{\circ} \mathrm{C}$, which makes it extremely difficult to manufacture native (Al) GaN substrates, unlike that of $\mathrm{Si}$. Consequently, high quality (Al)GaN substrates are extremely expensive. This has impeded the commercialization of the (Al)GaN technology and hindered the massproduction.

A solution to the above mentioned substrate-problem is to grow ( $\mathrm{Al}) \mathrm{GaN}$ heteroepitaxially on substrates with a reasonably-acceptable lattice match. Such substrates include sapphire (with mismatch of $16 \%$ with GaN and $12 \%$ with AlN), silicon carbide (3.1 and 1 $\%$, respectively) and $\mathrm{Si}(111)$ (17 and $22 \%$, respectively $)^{21-24}$. During the (Al)GaN epitaxy, the differences in the coefficients of thermal expansion (TCE) between ( $\mathrm{Al}) \mathrm{GaN}$ and the substrate also become important, as the growth occurs at high temperatures exceeding 1000 ${ }^{\circ} \mathrm{C}^{25}$. Whereas the lattice-mismatch leads to faults and defects in the as-grown (Al)GaN epitaxial layer ${ }^{\dagger}$, the TCE-mismatch causes film-cracking, wafer-bowing and breakage issues $^{26}$.

Therefore, although the above-mentioned substrates perform reasonably well in the epitaxial growth of $(\mathrm{Al}) \mathrm{GaN}$ layers, they do require pre-deposition of multiple buffer-layers to reduce the lattice- and TCE mismatch. The buffer-layer consists of single, or alternating stacks of $(\mathrm{Al}) \mathrm{GaN}$ layers, which can be up to several microns thick ${ }^{27-29}$. The use of the buffer-layers however poses restriction to the substrate-size due to the bowing and breakage issues $^{30}$. On the contrary, for the mass-production of III-Nitride semiconductor devices, large-sized substrates are essential to reduce the cost. Besides, just like the native (Al)GaN substrates, $\mathrm{SiC}$ and sapphire are also expensive in large sizes. Nowadays, (Al) GaN based devices find use in specific consumer and military-grade applications, such as in LEDs and HEMTs $^{30-31}$ typically fabricated on 2-inch substrates.

Combining the advanced Si-based process technology with the excellent material properties of $(\mathrm{Al}) \mathrm{GaN}$ electronics on one platform (for example, using $\mathrm{Si}(111)$ wafers as a

\footnotetext{
$\dagger$ These can be detrimental to the device performance, for instance, the internal quantum efficiency of
} LEDs. 
cost-effective substrate for the epitaxy of $(\mathrm{Al}) \mathrm{GaN}$ layers as well as exploiting the mature $\mathrm{Si}$ based technology) can, to a great extent, increase the functionality of integrated circuits and contribute to the More-than-Moore roadmap, enabling the combined (Al) $\mathrm{GaN}-\mathrm{Si}$ semiconductor devices to have a potential market share ${ }^{32}$ (see for example, references ${ }^{33-35}$ ). $\mathrm{Si}$ also provides excellent thermal and electrical conductivity, which allows to fabricate devices directly on $\mathrm{it}^{31}{ }^{36}$, therefore allowing to expand the scope of device design. Besides, the Si-based state-of-the-art fabrication facilities use large (up to 12 inch) substrates, which (Al)GaN technology can make use of. However, without the pre-deposition of buffer-layers, the devices could face serious performance-issues from the lattice-mismatch. In addition, the problem of the so-called 'meltback etch' (i.e., the formation of an amorphous interfacial layer between the $(\mathrm{Al}) \mathrm{GaN}$ layer and the $\mathrm{Si}$ substrate) is often encountered, if deposited without buffer layers, due to the high temperatures of epitaxy. These would subsequently degrade the crystalline (Al)GaN growth ${ }^{37}$. Some of these issues keep the (Al)GaN-on-Si technology still in the developing phase $\mathrm{s}^{35,38}$.

\subsubsection{From monocrystalline to polycrystalline}

The polycrystalline versions of the III-Nitrides have been largely ignored, because several material properties degrade from monocrystalline to polycrystalline films. However, research interest lies in determining which properties degrade and to what extent, to utilize such films for potential semiconductor devices. The deposition of polycrystalline films is much easier compared to the epitaxial counterparts. A growing number of device concepts and applications report thin sub-micron poly-(Al)GaN films in sensors ${ }^{39-40}$ LEDs $^{41-42}$, thin film transistors (TFT) ${ }^{43-46}$ and MEMS ${ }^{47-49}$. The motivation for using the poly-(Al)GaN semiconductors instead of their monocrystalline counterparts can be best compared to using poly- (and even amorphous) $\mathrm{Si}$ as lower-efficient, but cost-effective alternatives to monocrystalline Si in large-area displays, solar cells, etc. ${ }^{50}$. The relative lack of research on poly-( $\mathrm{Al}) \mathrm{GaN}$ is an opportunity to initiate further exploration, and this work is a step towards that.

The several advantages of the poly-III-Nitrides as prospective materials for electron devices originate from their relaxed deposition conditions. These comprise:

i. Choice of substrate: Due to the less-stringent demands on crystallinity, a large range of (including amorphous) substrates is possible. For example, growth of poly-GaN on $\mathrm{Si}(111)^{51}$, silica $^{52}$, sapphire ${ }^{53}$, glass ${ }^{54}$ and flexible polymer ${ }^{43}$ substrates have been reported. Besides, the buffer-layers are hardly required, allowing the poly-nitrides to be directly deposited on substrates, increasing (as mentioned before) their application range. For instance, poly-GaN on polymers have been used for flexible/wearable electronics ${ }^{44,55}$. 
ii. Deposition temperature: Poly-( $\mathrm{Al}) \mathrm{GaN}$ growth occurs at lower temperatures than that of epitaxy. In fact, poly-GaN films have even been prepared at room temperature ${ }^{54}, 56$. Low deposition temperatures expand the range of substrates and processes.

iii. Option of introducing foreign elements: Like all polycrystalline materials, the polynitrides allow for the addition of foreign elements (in quantities beyond doping levels) to realize composite materials. Such elements can be, for instance, located at the grain boundaries between the poly-crystals, opening up a phase-diagram between the poly-nitride and the introduced element. This enables further exploration of the composite, by changing the relative amounts of the elements. In this project, carbon (C) and gallium $(\mathrm{Ga})$ were introduced to poly-GaN, to realize a novel, composite ' $\mathrm{GaCN}$ ' layer with interesting optical properties $^{57}$.

iv. Deposition technique: Polycrystalline growth can be achieved with a variety of physicaland chemical vapour deposition techniques ${ }^{40,52,54}$, that are available at most fabrication facilities. These techniques can also be easily integrated in the process flow. The need for specialized epitaxy equipment (e.g., metalorganic chemical vapor deposition (MOCVD) and molecular beam epitaxy (MBE)) is eliminated.

Atomic layer deposition (ALD) is a chemical vapour deposition technique, that is becoming promising for obtaining poly-(Al)GaN. The aggressive miniaturization of microelectronic devices, reaching dimensions of a few nanometers, and the use of large substrates, demand that a modern thin-film deposition tool is able to offer (i) nanometer-level thickness control, (ii) excellent spatial uniformity, and (iii) excellent conformality on 3dimensional high-aspect-ratio structures, among others. For instance, some applications demanding such include: multi-gate field effect transistors (FETs) (e.g., FinFET and gate-allaround-FET), various volatile and non-volatile memories (e.g., dynamic random access memory or DRAM and flash memories), metal insulator metal (MIM) capacitors, etc.. Further, in advanced front-end and back-end CMOS processes, deposition of high- $k$ gatedielectric stacks (e.g., $\mathrm{Al}_{2} \mathrm{O}_{3}, \mathrm{HfO}_{2}$ and their alloys), metal gates (e.g., TiN, TaN) and metallization barrier- and nucleation-layers (e.g., TiN, W) are to be mentioned ${ }^{144-145}$. Besides, various ALD thin films are being used in MEMS applications, which enable the More-than-Moore roadmap. Not only the semiconductor industry, but several others such as photovoltaics, display, chemical, pharmaceutical, etc. employ thin film materials. ALD does an excellent job in satisfying the ( $\mathrm{i}-$ iii) stringent requirements. The excellent reviews by several authors testify the versatility of ALD in the miscellaneous applications ${ }^{58-73}$.

Among poly-III-Nitrides, thin ALD AlN films are used as passivation layers (in HEMTs, FETs, Schottky diodes and solid-state displays), high- $k$ gate-dielectrics, nucleation layers, memristors, coating of nanoparticles, and in various MEMS applications ${ }^{74-88}$. In comparison, the applications of ALD GaN films are less reported, and it is therefore believed 
that the technology is in a nascent phase. As a matter of fact, the number of ALD GaN reports, focussed mostly on the material properties, is growing in the recent times ${ }^{53,89-96}$. Some of the reported (and perceived) applications include TFTs $\mathrm{s}^{43-44}$, sensors, and photodetectors $^{97-99}$.

As a conclusion to this section, we re-iterate that the aim of this project is $\underline{\text { not }}$ to compete with crystalline (Al)GaN semiconductors in terms of their material properties, but to explore their polycrystalline counterparts as potential candidates for specific electronic applications using ALD - an appropriate modern deposition technique.

\subsection{Basic concepts of ALD}

ALD is a gas-to-solid chemical deposition technique which relies on film growth through self-limiting surface reactions, following the sequential (i.e., pulsed) introduction of precursors. It is essential that the precursors (i) chemisorb to the substrate, and (ii) react only on the substrate and not in the gas-phase. The latter leads to the loss of self-limiting reactions and may result in chemical vapor deposition (CVD) instead. To prevent the onset of CVD in an ALD process, pulses of an inert gas (e.g., $\mathrm{N}_{2}$, Ar) are introduced between the precursor pulses. The inert gas purges the unreacted precursors and the volatile reaction products, and ensures that the growth-reactions occur on the substrate only.

The film grows through a series of surface-reactions at every ALD cycle, in a selflimiting manner. This means that, the film-formation stops when all surface reactive sites have been occupied by the precursor molecules, which ideally ensures an atomic-level growth-control. Typically, each ALD cycle comprises of a pulse of reactant A, an inert gas purge, pulse of reactant $\mathrm{B}$, and a second inert gas purge (variation to this scheme have also been reported, such as, using three precursors instead of two, and using sub-cycles within a cycle $\left.{ }^{100-101}\right)$. The growth per ALD cycle is abbreviated as GPC. After every cycle, a monolayer film formation is ideally expected. ALD, by virtue of its self-limiting surfaceonly reactions, not only ensures an atomic-level growth, but also, offers an unprecedented level of spatial uniformity and film conformality ${ }^{102-104}$. Besides, the cycle-by-cycle growth nature facilitates easy alloying capability, which is useful for super-lattices ${ }^{105-106}$. The schematic of an ideal ALD process is shown in Fig. 1.1. 


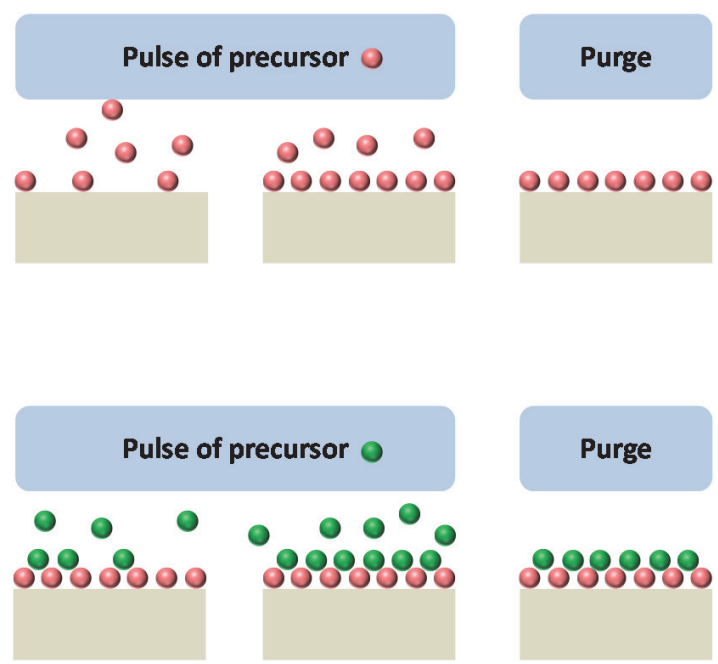

Fig. 1.1 Schematic of an ideal ALD cycle from precursor 1 (pink ball) and precursor 2 (green ball), showing film growth through saturated self-limiting surface reactions. To note: to maintain simplicity, chemical bonds are not shown.

In order to replicate Fig. 1.1, it is important that the precursors dose the substrate to saturation, i.e., chemisorb to all reactive surface sites. This results in the maximum GPC. The attainment of such regime is indicated by monitoring the GPC-change with the precursor pulse duration. Before attaining this regime, the film grows via under-saturated surface reactions and the GPC is sub-optimal, whereas beyond this regime, the GPC is independent of the pulse duration (Fig. 1.2 a). An efficient way to monitor these changes is by using an in-situ technique, such as spectroscopic ellipsometry $(\mathrm{SE})^{107}$.

Since ALD is a chemical process, temperature plays a key role in initiating surface reactions. At low temperatures, the GPC drops from its optimal value. At high temperatures, the GPC increases due to the premature precursor decomposition in the gas-phase (i.e., before reaching the substrate) or during the chemisorption. Both can lead to a loss of the selflimiting behaviour. Contradictory trends in GPC also exist ${ }^{102}$. For instance, low temperatures may condense the precursor on the substrate, inhibiting their chemisorption and leading to a loss of self-limiting reactions ${ }^{102}$. Likewise, high temperatures may cause precursor desorption after chemisorption. These trends are represented in Fig. $1.2 \mathbf{b}^{102}$. As shown, a temperature window for self-limiting surface reactions exist in most ALD processes, where the GPC is rather insensitive to temperature changes. Contrary to temperature, pressure does not play a key role in typical ALD processes, as long as the substrate is dosed optimally. 

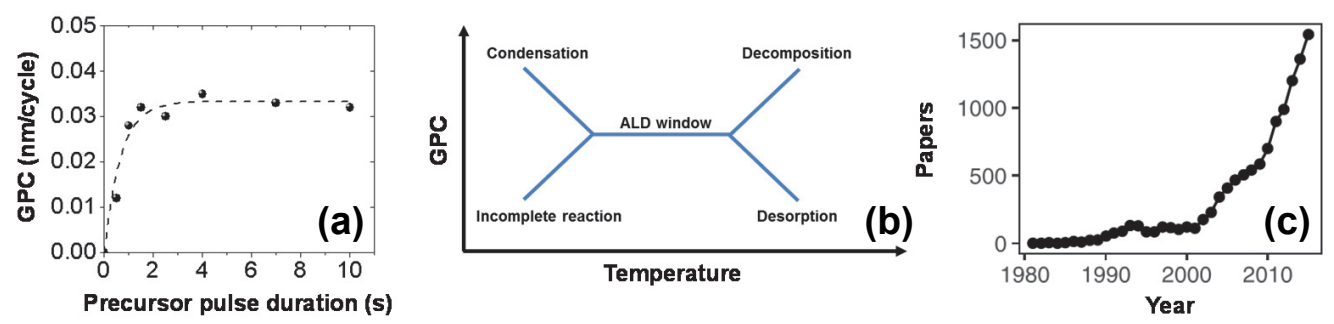

Fig. 1.2 Typical variation of GPC with (a) the precursor pulse duration, (b) temperature, showing the different regimes (original source: reference ${ }^{102}$ ). (c) The exponential growth of ALD reports over the last four decades (original source: reference ${ }^{108}$ ).

The initiation of the surface-reactions may occur purely by thermal activation (this is called 'thermal ALD') or by the assistance of radicals (and/or ions) produced by the dissociation of the precursors (this is called 'radical-enhanced ALD'). Radical-enhanced ALD is resorted to, when the surface reactions are thermodynamically unfavourable, in the temperature range where both the precursors can chemisorb in a self-limiting manner. Radicals unlock new chemical pathways at reduced temperatures. A plasma, generated using (one of) the precursors, is an effective source of radicals. Plasma-enhanced ALD (PEALD) has been successfully used to deposit a variety of elemental and compound thin films (see for example, a review by Knoops et al. and the references therein ${ }^{109}$ ).

For specific applications, the extraneous components associated with plasma such as ions, electrons and high-energy neutrals, may be undesired for the material properties. For such processes, an alternative, 'clean' manner of radical production is therefore required. One way to achieve it is by using a resistively-heated metallic filament as the radical source. It is typically heated between $1000-2000{ }^{\circ} \mathrm{C}$ and the precursor introduced along the filament. The molecules impinge on the heated filament surface and catalytically dissociate into radicals ${ }^{110}$, which then take part in the film-forming reactions. Such an ALD process is termed 'hot-wire assisted ALD' (HWALD) ${ }^{111}$. In this thesis, all three activation techniques (namely, thermal, plasma and hot-wire) have been explored for the ALD of (Al)GaN films.

To conclude the discussion on ALD, the remarkable role of this deposition technique not only in the semiconductor industry but in several others, is confirmed by the exponential rise of ALD publications over the past few decades (Fig. 1.2 c) ${ }^{108}$. The same holds for the successful realization of ALD recipes to enable various elemental and compound films, as represented by the so-called 'ALD periodic table' (Fig. 1.3) ${ }^{112}$. 


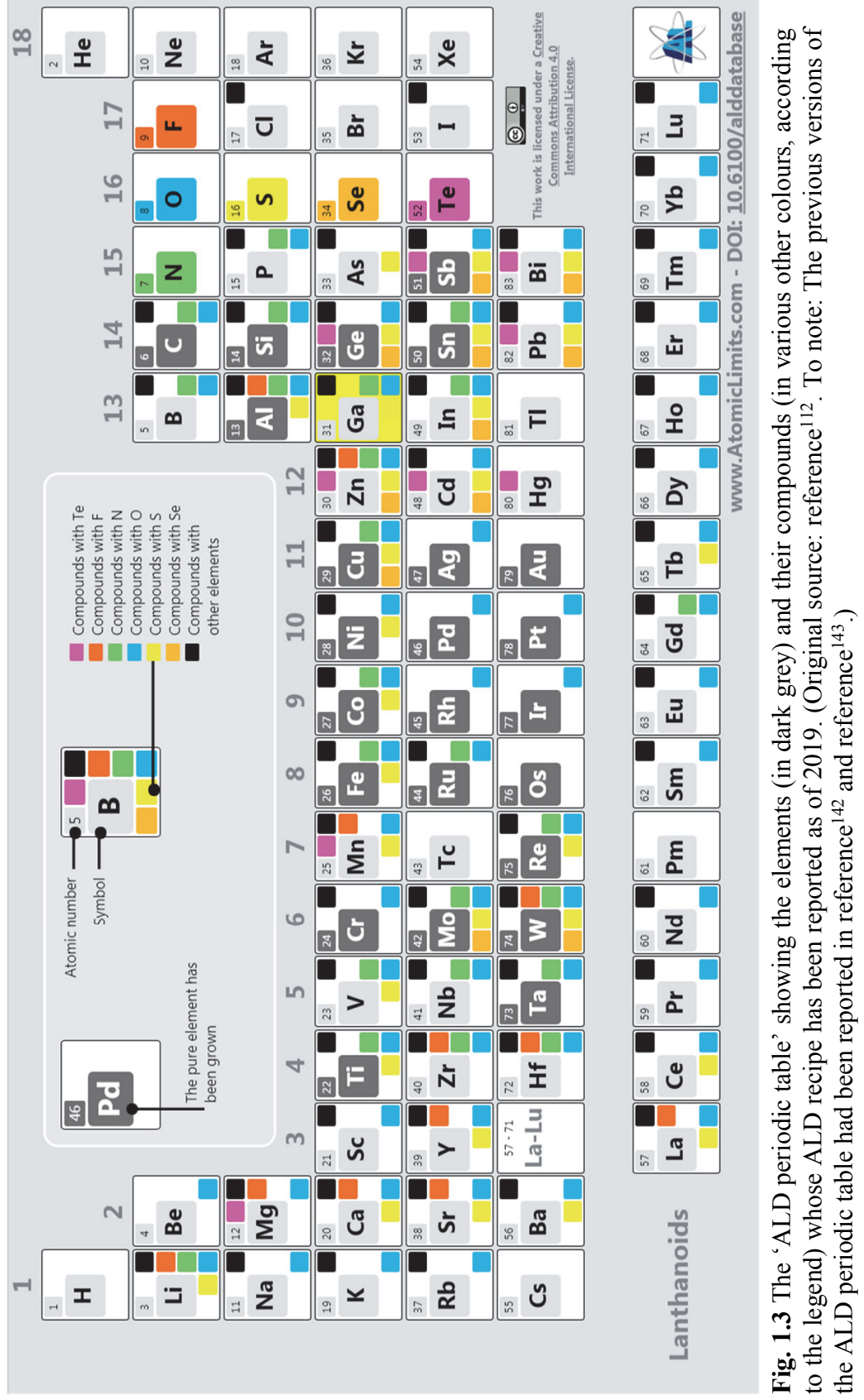




\subsubsection{Precursor choice for (AI)GaN ALD}

Prior to the commencement of the project, a literature study was done to identify the precursors for the ALD of GaN and AlN, as listed in Table 1.1. The metal-containing precursor is classified into metalorganic and inorganic. Chloride precursors are typically inorganic. The nitrogen-containing ( $\mathrm{N}$-containing) precursors include $\mathrm{NH}_{3}, \mathrm{~N}_{2}$ and $\mathrm{N}_{2}-\mathrm{H}_{2}$ mixture.

Whereas, with chlorides and $\mathrm{NH}_{3}$ (or $\mathrm{N}_{2}-\mathrm{H}_{2}$ mixture), it is possible to deposit (Al)GaN thermally, the process occurs at high temperatures, exceeding $500{ }^{\circ} \mathrm{C}^{113-114}$. This is due to the chemical stability of the N-containing precursor (to note: reports on (Al)GaN ALD using more-reactive precursors, e.g., hydrazine, are only recently appearing $\left.{ }^{115}\right)$. The chemical stability also limits the use of metalorganic precursors in purely thermal mode, since they dissociate at the high temperatures required to dissociate the $\mathrm{N}$-containing precursor (for example, whereas $\mathrm{NH}_{3}$ starts to dissociate on the surface beyond $600{ }^{\circ} \mathrm{C}^{116}$, TMG already dissociates at $\sim 150{ }^{\circ} \mathrm{C}^{117}$ ). Therefore, a plasma or hot-wire is employed, which facilitates the ALD at temperatures lower than the dissociation-threshold of the metalorganics.

Table 1.1 Summary of $\mathrm{Al}(\mathrm{Ga})$ - and N-containing precursors in (Al)GaN ALD literature.

\begin{tabular}{|c|c|c|c|}
\hline \multicolumn{2}{|c|}{ GaN } & \multicolumn{2}{|c|}{ AIN } \\
\hline Ga-precursor & N-precursor & Al-precursor & N-precursor \\
\hline $\begin{array}{l}\text { Metalorganic } \\
\text { TMG }^{51,56-57,92-93,96,118-121} \text {, } \\
\text { TEG }^{92,122-125}\end{array}$ & $\begin{array}{l}\mathrm{NH}_{3}{ }^{57,113-114,119-121,126} \\
\mathrm{NH}_{3} \text {-plasma } \\
\mathrm{N}_{2}-\mathrm{H}_{2} \text {-plasma } 92-93, \\
122-123\end{array}$ & $\begin{array}{l}\text { Metalorganic } \\
\text { TMA }^{111,127-132} \text {, } \\
\text { TEA }^{133}, \\
\text { DMAA }^{134}, \text { TMAA }^{135}\end{array}$ & $\begin{array}{l}\mathrm{NH}_{3}{ }^{127-129,}, 131,133-135 \\
\mathrm{NH}_{3} \text {-plasma } \\
136\end{array}$ \\
\hline $\begin{array}{l}\text { Inorganic (chloride) } \\
\mathrm{GaCl}^{126} \\
\mathrm{GaCl}_{3}{ }^{113-114}\end{array}$ & $\begin{array}{l}\mathrm{N}_{2} \text {-plasma } \\
\mathrm{NH}_{3} \text {-hot wire } \\
\mathrm{NH}_{3}+\text { atomic- }^{118}+e^{56}\end{array}$ & $\begin{array}{l}\text { Inorganic } \\
\text { (chloride) } \\
\mathrm{AlCl}_{3}{ }^{128,136}\end{array}$ & $\begin{array}{l}\mathrm{N}_{2}-\mathrm{H}_{2} \text {-plasma } \\
\mathrm{NH}_{3} \text {-hot wire }\end{array}$ \\
\hline
\end{tabular}

(TMG - Trimethylgallium; TEG - Triethylgallium; TMA - Trimethylaluminum;

TEA - Triethylaluminum; DMAA - Dimethylamine alane; TMAA - Trimethylamine alane)

For this project, the precursors chosen for GaN ALD were TMG $(99.9999 \%$ electronic grade) and $\mathrm{NH}_{3}(99.999 \%)$; likewise, for AlN the precursors were TMA ( $99.9999 \%$ electronic grade) and $\mathrm{NH}_{3}$. The choices were motivated by:

i. The easy-availability of these precursors due to their industrial usage, e.g., in MOCVD.

ii. The ease of delivery of metalorganic precursors in general, compared to the chloride counterparts. This is due to the formers' significantly higher vapor pressure, which 
eliminates the need for supply- and gas-line heating in the reactor. Besides, the chlorides also tend to etch the reactor hardware (from the corrosive $\mathrm{HCl}$ by-product) and cause incorporation of chlorine in the growing film ${ }^{93}$.

iii. The prospect of achieving an industrially-acceptable low-temperature ALD process of (Al) GaN using metalorganic precursors, with or without radical-assistance. Such a process was indeed realized through a hitherto-unreported chemical route, and serves as an important finding of this thesis.

\subsubsection{ALD hardware}

An in-house designed and constructed ALD-CVD cluster system (Fig. 1.4 a)

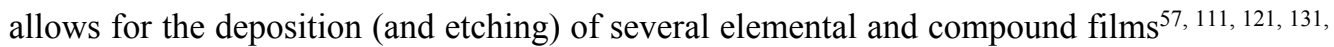
${ }^{137-141}$. The system consists of four reactors sharing a common loadlock, the latter evacuated by a turbo-molecular pump (Pfeiffer Vaccuum), and reaching base pressure of $10^{-7}$ mbar. The loadlock facilitates the transfer of wafer between the reactors without vacuum break. (Al) $\mathrm{GaN}$ is deposited in the right-most reactor, as indicated.

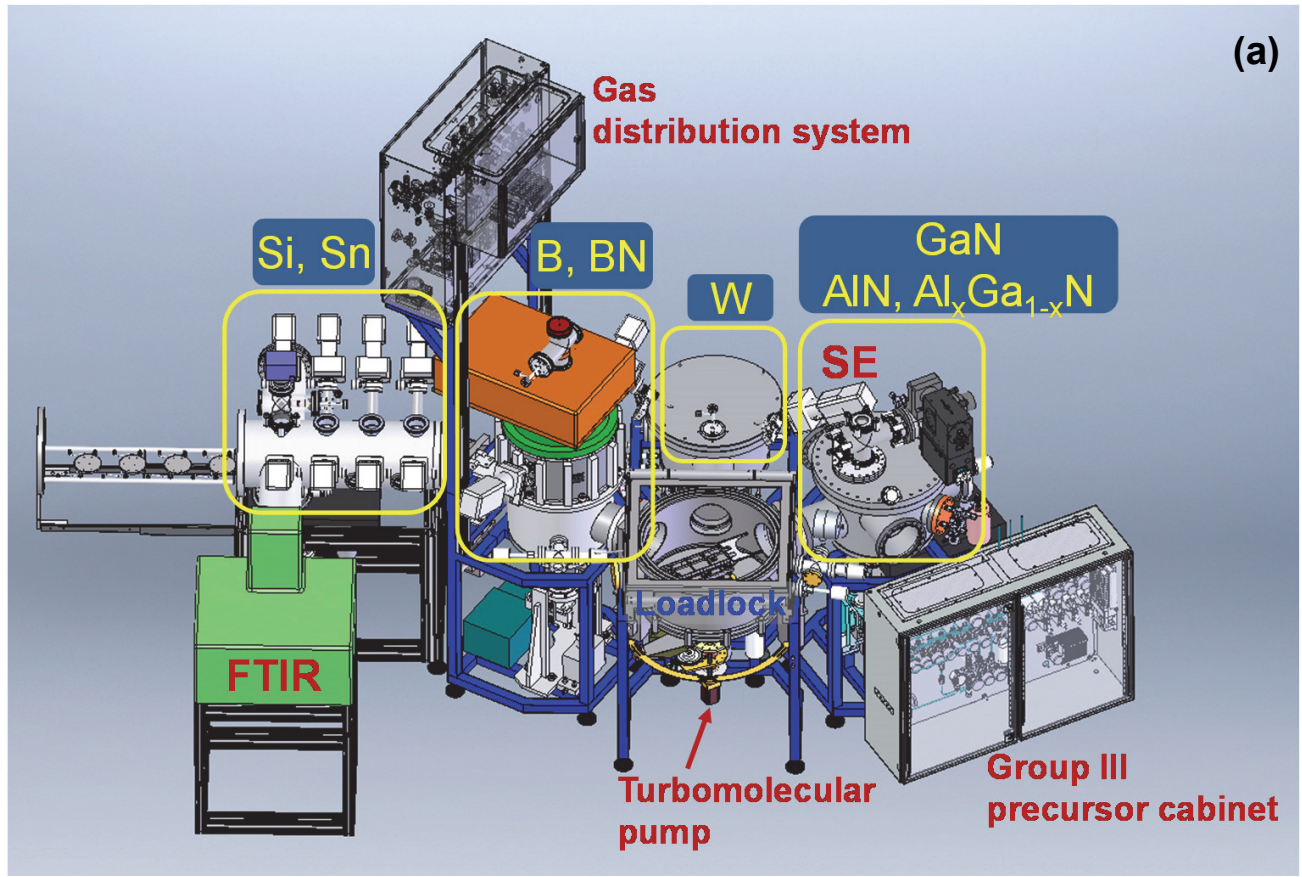

Fig. 1.4 (a) Schematic of the home-built ALD-CVD cluster system, facilitating the deposition of multiple films. The materials associated with each reactor, and the in-situ monitoring systems (e.g., spectroscopic ellipsometer (SE) and Fourier transform infrared spectrometer (FTIR)) are indicated. 


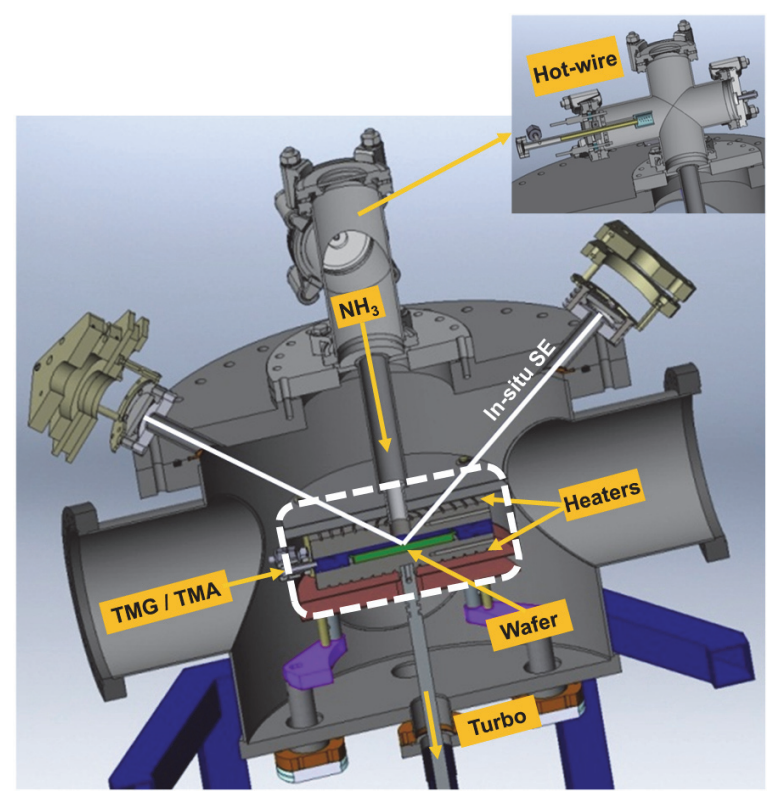

(b)

Fig. 1.4 (b) Cross-section schematic of the (Al)GaN ALD reactor. The dotted white box shows the inner reactor. Inset: the vertical flange is zoomed in to show the location of the hot-wire.

Fig. 1.4 b shows the cross-section schematic of the (Al) GaN reactor. It consists of an outer cold-wall reactor, which surrounds a small-volume $\left(32 \mathrm{~cm}^{3}\right)$ hot-wall inner reactor, as indicated by the dotted white box. The hot-wall solution reduces the residence time of the gas molecules adsorbed to the reactor walls, and the small volume aids in efficient purging. Both factors are conducive to ALD. The precursors are introduced into the inner reactor as shown in Fig. 1.4 b. The reactor is connected to a turbo-molecular pump, which ensures a base pressure of $10^{-7}$ mbar. A computer-controlled throttle valve establishes the desired pressure during ALD, ranging from $10^{-3}$ to $10^{1} \mathrm{mbar}$. The inner reactor is heated by resistive heating elements located at the roof and the base. A thermocouple monitors the chuck temperature.

From the precursor flow directions indicated in Fig. 1.4 b (TMG and TMA are introduced laterally, and $\mathrm{NH}_{3}$ is introduced vertically, unless otherwise mentioned), it becomes apparent that the reactor is a hybrid of cross-flow and shower-head designs. The precursors are delivered using an inert carrier gas (Argon, 99.999\%), using high-speed ALD valves (Swagelok) of $0.1 \mathrm{~s}$ time resolution. The vertical flange, where $\mathrm{NH}_{3}$ is introduced (see inset), has space for installing the hot-wire or a plasma-generator. 
The reactor is equipped with a Woollam M-2000 spectroscopic ellipsometer (SE), operating in the wavelength range $245-1688 \mathrm{~nm}$, and acquiring data every $2.5 \mathrm{~s}$ (unless stated otherwise). The ellipsometric analysis is performed with the CompleteEASE software from J.A.Woollam. The in-situ SE enables monitoring the film growth and the optical constant changes in real time, during the ALD. The wafer temperature can be in-situ monitored as well (see Appendix 1).

\subsection{Thesis outline}

Chapter 2 addresses the PEALD of (Al)GaN films, and reports the material properties as characterized with in-situ and ex-situ techniques. The ALD window is determined for AlN in terms of the precursor duration and the process temperature. For both AlN and GaN, the aim is to increase the preferential formation of the wurtzitic (002) crystal planes. To achieve this, the recipe and substrate conditions are optimized in terms of (i) plasma power, (ii) substrate pre-treatment, (iii) growth on seed layers, and (iv) plasma composition. To understand the role of plasma composition, optical emission spectroscopy (OES) of the $\mathrm{NH}_{3}-$ Ar plasma is performed.

Chapter 3 explores the novel HWALD technique for depositing (Al)GaN films. The radical generation by the HW and their delivery to the substrate are investigated, by positioning the HW both in and outside the line-of-sight (L-O-S) to the substrate. During the HWALD of AIN, significant oxygen contamination occurs, resulting in an oxynitride $\left(\mathrm{AlO}_{\mathrm{x}} \mathrm{N}_{\mathrm{y}}\right)$ ring at the center of the wafer and $\mathrm{AlN}$ formation outside the ring. During the targeted HWALD of GaN, Ga droplets are formed. Probable causes behind these observations are proposed.

Chapter 4 explores the novel composite $\mathrm{GaCN}$ thin film, that consists of nanoinclusions of polycrystalline $\mathrm{GaN}$, with $\mathrm{C}$ and $\mathrm{Ga}$ clusters located between the poly-crystals. The composite is prepared by thermal ALD and is viewed as the Ga-analogue to other carbonitrides such as $\mathrm{BCN}$ and SiCN. The phase-segregated nature of the GaCN composite is concluded after a detailed material characterization. Varying the temperature and the $\mathrm{NH}_{3}$ partial pressure, composites with a wide range of $\mathrm{Ga}, \mathrm{C}$ and $\mathrm{N}$ are prepared, and their optical properties are measured. An explanation behind the impact of carbon on the refractive indices is presented.

Chapter 5 introduces thermal ALD of polycrystalline GaN films from TMG and $\mathrm{NH}_{3}$ precursors, at a modest temperature of $400{ }^{\circ} \mathrm{C}$. The ALD is facilitated by (i) the (speculated) existence of a novel chemical complex - the TMG: $\mathrm{NH}_{3}$ surface adduct, and (ii) conversion of the adduct into $\mathrm{Ga}-\mathrm{NH}_{2}-\mathrm{Ga}$ linkages, signifying the formation of a $\mathrm{GaN}$ unit. A GPC of $0.1 \mathrm{~nm} /$ cycle is achieved, and it is revealed to be a strong function of the $\mathrm{NH}_{3}$ 
pulse duration and the partial pressure. Such pressure dependence is rather atypical in ALD, and is the key to growing GaN layers thermally.

Chapter 6 explores the inherent substrate-selective growth nature of thermal ALD $\mathrm{GaN}$. The presence of $-\mathrm{NH}_{2}$ terminations on a substrate causes an instant growth (i.e., without an incubation period). On the contrary, the presence of other terminations (such as $-\mathrm{H}$ and $-\mathrm{OH}$ ) induces a long incubation period. The role of the $-\mathrm{NH}_{2}$ terminations is explored in low-pressure and high-pressure GaN ALD. The first results towards areaselective ALD of GaN are presented.

Chapter 7 addresses the important aspect of ALD - the coalescence (i.e., closure) of thin films. This may not be guaranteed from the early stages of deposition, although ALD apparently implies self-limiting surface reactions. Thermal ALD of AIN layers is selected as a case-study. Tests for coalescence are performed (i) electrically, using Metal-InsulatorMetal (M-I-M) test structures and (ii) optically, with in-situ SE monitoring. The existence of pre-coalesced, sub-5 nm AlN clusters is also confirmed by transmission electron microscopy (TEM) imaging.

Chapter 8, the concluding chapter, provides a summary of all the individual chapters and gives recommendations for future work in the direction of ALD of III-Nitride materials. 


\section{References}

1. O. Ambacher, Journal of Physics D: Applied Physics, 1998, 31, 2653.

2. P. Kung and M. Razeghi, Opto-electronics Review, 2000, 8, 201-239.

3. I. M. Watson, Coordination Chemistry Reviews, 2013, 257, 2120-2141.

4. C. Abels, V. Mastronardi, F. Guido, T. Dattoma, A. Qualtieri, W. Megill, M. De Vittorio and F. Rizzi, Sensors, 2017, 17, 1080.

5. S. Strite and H. Morkoç, Journal of Vacuum Science \& Technology B: Microelectronics and Nanometer Structures Processing, Measurement, and Phenomena, 1992, 10, 1237-1266.

6. O. Ambacher, B. Foutz, J. Smart, J. Shealy, N. Weimann, K. Chu, M. Murphy, A. Sierakowski, W. Schaff and L. Eastman, Journal of Applied Physics, 2000, 87, 334-344.

7. U. K. Mishra, P. Parikh and Y.-F. Wu, Proceedings of the IEEE, 2002, 90, 1022-1031.

8. S. Nakamura and M. R. Krames, Proceedings of the IEEE, 2013, 101, 2211-2220.

9. D. W. Runton, B. Trabert, J. B. Shealy and R. Vetury, IEEE Microwave Magazine, 2013, 14, 82-93.

10. I. M. Ross, Proceedings of the IEEE, 1998, 86, 7-28.

11. L. Łukasiak and A. Jakubowski, Journal of Telecommunications and Information technology, 2010, 3-9.

12. J. S. Kilby, IEEE Transactions on Electron Devices, 1976, 23, 648-654.

13. D. Kahng, Solid State Device Research Conf., Pittsburgh, PA. June 1960.

14. S. Hofstein and F. Heiman, Proceedings of the IEEE, 1963, 51, 1190-1202.

15. F. M. Wanlass and C. T. Sah, in Semiconductor Devices: Pioneering Papers, World Scientific, 1991, pp. 637-638.

16. R. E. Kerwin, D. L. Klein and J. C. Sarace, U.S. Patent No. 3,475,234, 1969.

17. G. E. Moore, Electron Devices Meeting, 1975, 21, 11-13.

18. E. E. Loebner, IEEE Transactions on Electron Devices, 1976, 23, 675-699.

19. J. Pankove, E. Miller and J. Berkeyheiser, in Luminescence of Crystals, Molecules, and Solutions, Springer, 1973, pp. 426-430.

20. W. Zulehner, Journal of Crystal Growth, 1983, 65, 189-213.

21. K. Gurnett and T. Adams, III-Vs Review, 2006, 19, 39-41. 
22. R. Vispute, J. Narayan, H. Wu and K. Jagannadham, Journal of Applied Physics, 1995, 77, 4724-4728.

23. A. Dadgar, M. Poschenrieder, J. Bläsing, O. Contreras, F. Bertram, T. Riemann, A. Reiher, M. Kunze, I. Daumiller and A. Krtschil, Journal of Crystal Growth, 2003, 248, 556-562.

24. S. Kukushkin, A. Osipov, V. Bessolov, B. Medvedev, V. Nevolin and K. Tcarik, Reviews on Advanced Materials Science, 2008, 17, 1-32.

25. L. Liu and J. H. Edgar, Materials Science and Engineering: R: Reports, 2002, 37, 61-127.

26. D. Zhu, D. Wallis and C. Humphreys, Reports on Progress in Physics, 2013, 76, 106501.

27. H. Amano, N. Sawaki, I. Akasaki and Y. Toyoda, Applied Physics Letters, 1986, 48, 353355.

28. A. Able, W. Wegscheider, K. Engl and J. Zweck, Journal of Crystal Growth, 2005, 276, 415418.

29. K. Dovidenko, S. Oktyabrsky, J. Narayan and M. Razeghi, Journal of Applied Physics, 1996, 79, 2439-2445.

30. H. Amano, Y. Baines, E. Beam, M. Borga, T. Bouchet, P. R. Chalker, M. Charles, K. J. Chen, N. Chowdhury and R. Chu, Journal of Physics D: Applied Physics, 2018, 51, 163001.

31. G. Li, W. Wang, W. Yang, Y. Lin, H. Wang, Z. Lin and S. Zhou, Reports on Progress in Physics, 2016, 79, 056501.

32. N. Bardsley, S. Bland, D. Chwastyk, C. Monasterio, L. Pattison, M. Pattison, F. Welsh and M. Yamada, US Department of Energy, Washington, DC, 2013.

33. M. Van Hove, S. Boulay, S. R. Bahl, S. Stoffels, X. Kang, D. Wellekens, K. Geens, A. Delabie and S. Decoutere, IEEE Electron Device Letters, 2012, 33, 667-669.

34. H.-S. Lee, D. S. Lee and T. Palacios, IEEE Electron Device Letters, 2011, 32, 623-625.

35. T. Li, M. Mastro and A. Dadgar, III-V Compound Semiconductors: Integration with SiliconBased Microelectronics, CRC press, 2010.

36. Y. Zhang, D. Piedra, M. Sun, J. Hennig, A. Dadgar, L. Yu and T. Palacios, IEEE Electron Device Letters, 2017, 38, 248-251.

37. H. Ishikawa, K. Yamamoto, T. Egawa, T. Soga, T. Jimbo and M. Umeno, Journal of Crystal Growth, 1998, 189, 178-182.

38. A. Dadgar, Physica Status Solidi (B), 2015, 252, 1063-1068.

39. O. Siegmund, J. Vallerga, J. McPhate, J. Malloy, A. Tremsin, A. Martin, M. Ulmer and B. Wessels, Nuclear Instruments and Methods in Physics Research Section A: Accelerators, Spectrometers, Detectors and Associated Equipment, 2006, 567, 89-92. 
40. S. Yagi, Applied Physics Letters, 2000, 76, 345-347.

41. D. Bour, N. Nickel, C. Van de Walle, M. Kneissl, B. Krusor, P. Mei and N. Johnson, Applied Physics Letters, 2000, 76, 2182-2184.

42. J. H. Choi, H. Y. Ahn, Y. S. Lee, K. Park, T.-H. Kim, K. S. Cho, C. W. Baik, S. I. Kim, H. Yoo and E. H. Lee, Journal of Materials Chemistry, 2012, 22, 22942-22948.

43. S. Bolat, Z. Sisman and A. Okyay, Applied Physics Letters, 2016, 109, 233504.

44. S. Bolat, C. Ozgit-Akgun, B. Tekcan, N. Biyikli and A. Okyay, Applied Physics Letters, 2014, 104, 243505.

45. R. Chen, W. Zhou, M. Zhang and H. S. Kwok, IEEE Electron Device Letters, 2012, 33, 1282-1284.

46. C. Liu, S. Liu, S. Huang and K. J. Chen, IEEE Electron Device Letters, 2013, 34, 1106-1108.

47. S. Trolier-McKinstry and P. Muralt, Journal of Electroceramics, 2004, 12, 7-17.

48. V. M. Mastronardi, L. Ceseracciu, F. Guido, F. Rizzi, A. Athanassiou, M. De Vittorio and S. Petroni, Applied Physics Letters, 2015, 106, 162901.

49. K. Tonisch, V. Cimalla, C. Foerster, H. Romanus, O. Ambacher and D. Dontsov, Sensors and Actuators A: Physical, 2006, 132, 658-663.

50. R. A. Street, Technology and Applications of Amorphous Silicon, Springer Science \& Business Media, 2013.

51. G. Gupta, S. Banerjee, S. Dutta, A. A. I. Aarnink, J. Schmitz, A. Y. Kovalgin and R. J. E. Hueting, Journal of Applied Physics, 2018, 124, 084503.

52. R. Chen, W. Zhou and H. Sing Kwok, Applied Physics Letters, 2012, 100, 02211.

53. S. Kizir, A. Haider and N. Biyikli, Journal of Vacuum Science \& Technology A: Vacuum, Surfaces, and Films, 2016, 34, 041511.

54. K. P. Biju, A. Subrahmanyam and M. K. Jain, Journal of Crystal Growth, 2009, 311, 22752280.

55. S. Y. Lee, K.-I. Park, C. Huh, M. Koo, H. G. Yoo, S. Kim, C. S. Ah, G. Y. Sung and K. J. Lee, Nano Energy, 2012, 1, 145-151.

56. J. K. Sprenger, A. S. Cavanagh, H. Sun, K. J. Wahl, A. Roshko and S. M. George, Chemistry of Materials, 2016, 28, 5282-5294.

57. S. Banerjee, A. J. Onnink, S. Dutta, A. A. I. Aarnink, D. J. Gravesteijn and A. Y. Kovalgin, The Journal of Physical Chemistry C, 2018, 122, 29567-29576.

58. C. Detavernier, J. Dendooven, S. P. Sree, K. F. Ludwig and J. A. Martens, Chemical Society Reviews, 2011, 40, 5242-5253. 
59. Q. Xie, S. Deng, M. Schaekers, D. Lin, M. Caymax, A. Delabie, X.-P. Qu, Y.-L. Jiang, D. Deduytsche and C. Detavernier, Semiconductor Science and Technology, 2012, 27, 074012.

60. H. Van Bui, F. Grillo and J. Van Ommen, Chemical Communications, 2017, 53, 45-71.

61. J. Van Delft, D. Garcia-Alonso and W. Kessels, Semiconductor Science and Technology, 2012, 27, 074002.

62. A. Mackus, A. Bol and W. Kessels, Nanoscale, 2014, 6, 10941-10960.

63. H. Kim and W.-J. Maeng, Thin Solid Films, 2009, 517, 2563-2580.

64. O. Graniel, M. Weber, S. Balme, P. Miele and M. Bechelany, Biosensors and Bioelectronics, 2018.

65. H. Kim, Journal of Vacuum Science \& Technology B: Microelectronics and Nanometer Structures Processing, Measurement, and Phenomena, 2003, 21, 2231-2261.

66. M. Knez, K. Nielsch and L. Niinistö, Advanced Materials, 2007, 19, 3425-3438.

67. S. Seo, S. Jeong, H. Park, H. Shin and N.-G. Park, Chemical Communications, 2019, 55, 2403-2416.

68. F. Zaera, Chemical Society Reviews, 2013, 42, 2746-2762.

69. B. Yan, X. Li, Z. Bai, X. Song, D. Xiong, M. Zhao, D. Li and S. Lu, Journal of Power Sources, 2017, 338, 34-48.

70. J. A. Singh, N. Yang and S. F. Bent, Annual Review of Chemical and Biomolecular Engineering, 2017, 8, 41-62.

71. C. Marichy, M. Bechelany and N. Pinna, Advanced Materials, 2012, 24, 1017-1032.

72. W. Hao, C. Marichy and C. Journet, 2D Materials, 2018, 6, 012001.

73. N. Biyikli and A. Haider, Semiconductor Science and Technology, 2017, 32, 093002.

74. H. Kim, N. Do Kim, S. C. An and B. J. Choi, Journal of Materials Science: Materials in Electronics, 2018, 29, 17508-17516.

75. J.-J. Zhu, X.-H. Ma, Y. Xie, B. Hou, W.-W. Chen, J.-C. Zhang and Y. Hao, IEEE Transactions on Electron Devices, 2015, 62, 512-518.

76. S. Huang, Q. Jiang, S. Yang, C. Zhou and K. J. Chen, IEEE Electron Device Letters, 2012, 33, 516-518.

77. T.-E. Hsieh, E. Y. Chang, Y.-Z. Song, Y.-C. Lin, H.-C. Wang, S.-C. Liu, S. Salahuddin and C. C. Hu, IEEE Electron Device Letters, 2014, 35, 732-734. 
78. D. Kueck, P. Leber, A. Schmidt, G. Speranza and E. Kohn, Diamond and Related Materials, 2010, 19, 932-935.

79. E. Schilirò, F. Giannazzo, C. Bongiorno, S. Di Franco, G. Greco, F. Roccaforte, P. Prystawko, P. Kruszewski, M. Leszczyński and M. Krysko, Materials Science in Semiconductor Processing, 2019, 97, 35-39.

80. C.-I. Wang, T.-J. Chang, C.-Y. Wang, Y.-T. Yin, J.-J. Shyue, H.-C. Lin and M.-J. Chen, RSC Advances, 2019, 9, 592-598.

81. H. Kim, Y. Kwon and B. J. Choi, Thin Solid Films, 2019, 670, 41-45.

82. M. Peng, X. Zheng, S. Liu, H. Wei, Y. He, M. Li, Y. An, Y. Song and P. Qiu, Nanoscale, $2019,11,3710-3717$.

83. H. Seppänen, I. Kim, J. Etula, E. Ubyivovk, A. Bouravleuv and H. Lipsanen, Materials, 2019, 12, 406.

84. B. J. Choi, J. J. Yang, M.-X. Zhang, K. J. Norris, D. A. Ohlberg, N. P. Kobayashi, G. Medeiros-Ribeiro and R. S. Williams, Applied Physics A, 2012, 109, 1-4.

85. Y. Zhou, D. M. King, J. Li, K. S. Barrett, R. B. Goldfarb and A. W. Weimer, Industrial \& Engineering Chemistry Research, 2010, 49, 6964-6971.

86. L.-Y. Zhu, J.-G. Yang, K. Yuan, H.-Y. Chen, T. Wang, H.-P. Ma, W. Huang, H.-L. Lu and D. W. Zhang, APL Materials, 2018, 6, 121109.

87. P. Sippola, A. Pyymaki Perros, O. M. Ylivaara, H. Ronkainen, J. Julin, X. Liu, T. Sajavaara, J. Etula, H. Lipsanen and R. L. Puurunen, Journal of Vacuum Science \& Technology A: Vacuum, Surfaces, and Films, 2018, 36, 051508.

88. N. Nepal, R. Goswami, S. Qadri, N. Mahadik, F. Kub and C. Eddy Jr, Scripta Materialia, 2014, 93, 44-47.

89. N. Gungor and M. Alevli, Journal of Vacuum Science \& Technology A: Vacuum, Surfaces, and Films, 2018, 36, 021514.

90. S.-J. Liu, Y.-F. He, H.-Y. Wei, P. Qiu, Y.-M. Song, Y.-L. An, A. Rehman, M.-Z. Peng and X.-H. Zheng, Chinese Physics B, 2019, 28, 026801.

91. J. Gong, S. Liu, Y. He, X. Feng, X. Xia, Z. Quan and L. Wang, Applied Physics Letters, 2017, 111, 122103.

92. M. Alevli, A. Haider, S. Kizir, S. A. Leghari and N. Biyikli, Journal of Vacuum Science \& Technology A: Vacuum, Surfaces, and Films, 2016, 34, $01 \mathrm{~A} 137$.

93. C. Ozgit-Akgun, E. Goldenberg, A. K. Okyay and N. Biyikli, Journal of Materials Chemistry C, 2014, 2, 2123-2136.

94. M. Alevli, N. Gungor, A. Haider, S. Kizir, S. A. Leghari and N. Biyikli, Journal of Vacuum Science \& Technology A: Vacuum, Surfaces, and Films, 2016, 34, 01A125. 
95. P. Motamedi and K. Cadien, RSC Advances, 2015, 5, 57865-57874.

96. P. Pansila, K. Kanomata, M. Miura, B. Ahmmad, S. Kubota and F. Hirose, Applied Surface Science, 2015, 357, 1920-1927.

97. B. Tekcan, C. Ozgit-Akgun, S. Bolat, N. Biyikli and A. K. Okyay, Optical Engineering, 2014, 53, 107106.

98. C. Ozgit-Akgun, F. Kayaci, S. Vempati, A. Haider, A. Celebioglu, E. Goldenberg, S. Kizir, T. Uyar and N. Biyikli, Journal of Materials Chemistry C, 2015, 3, 5199-5206.

99. S. Bolat, B. Tekcan, C. Ozgit-Akgun, N. Biyikli and A. K. Okyay, Journal of Vacuum Science \& Technology A: Vacuum, Surfaces, and Films, 2015, 33, $01 \mathrm{~A} 143$.

100. T. Cheon, S.-H. Choi, S.-H. Kim and D.-H. Kang, Electrochemical and Solid-State Letters, 2011, 14, D57-D61.

101. M. Donders, W. Arnoldbik, H. Knoops, W. Kessels and P. Notten, Journal of The Electrochemical Society, 2013, 160, A3066-A3071.

102. S. M. George, Chemical Reviews, 2009, 110, 111-131.

103. R. W. Johnson, A. Hultqvist and S. F. Bent, Materials Today, 2014, 17, 236-246.

104. R. L. Puurunen, Journal of Applied Physics, 2005, 97, 9.

105. C. R. Eddy Jr, N. Nepal, J. K. Hite and M. A. Mastro, Journal of Vacuum Science \& Technology A: Vacuum, Surfaces, and Films, 2013, 31, 058501.

106. N. Nepal, C. R. Eddy Jr, N. A. Mahadik, S. B. Qadri and M. J. Mehl, U.S. Patent No. 9,773,666, 2017.

107. E. Langereis, S. Heil, H. Knoops, W. Keuning, M. Van de Sanden and W. Kessels, Journal of Physics D: Applied Physics, 2009, 42, 073001.

108. E. Alvaro and A. Yanguas-Gil, PloS one, 2018, 13, e0189137.

109. H. C. Knoops, T. Faraz, K. Arts and W. M. M. Kessels, Journal of Vacuum Science \& Technology A: Vacuum, Surfaces, and Films, 2019, 37, 030902.

110. J. N. Smith Jr and W. L. Fite, The Journal of Chemical Physics, 1962, 37, 898-904.

111. A. Y. Kovalgin, M. Yang, S. Banerjee, R. O. Apaydin, A. A. I. Aarnink, S. Kinge and R. A. M. Wolters, Advanced Materials Interfaces, 2017, 4, 1700058.

112. W. Kessels, https://www.atomiclimits.com/alddatabase, 2019.

113. O. H. Kim, D. Kim and T. Anderson, Journal of Vacuum Science \& Technology A: Vacuum, Surfaces, and Films, 2009, 27, 923-928. 
114. H. Tsuchiya, M. Akamatsu, M. Ishida and F. Hasegawa, Japanese Journal of Applied Physics, 1996, 35, L748.

115. A. Abdulagatov, S. M. Ramazanov, R. Dallaev, E. Murliev, D. Palchaev, M. K. Rabadanov and I. Abdulagatov, Russian Microelectronics, 2018, 47, 118-130.

116. M. Mesrine, N. Grandjean and J. Massies, Applied Physics Letters, 1998, 72, 350-352.

117. M. J. Bronikowski and R. J. Hamers, Surface Science, 1996, 348, 311-324.

118. M. Alevli and N. Gungor, Journal of Vacuum Science \& Technology A: Vacuum, Surfaces, and Films, 2018, 36, $01 \mathrm{~A} 110$.

119. H. Wang, S. Huang, T. Yan, J. Gong, T. Lin and Y. Chen, Materials Science and Engineering: B, 1999, 57, 218-223.

120. N. Karam, T. Parodos, P. Colter, D. McNulty, W. Rowland, J. Schetzina, N. El-Masry and S. M. Bedair, Applied Physics Letters, 1995, 67, 94-96.

121. S. Banerjee, and A. Y. Kovalgin, ECS Transactions, 2018, 86, 21-29.

122. A. Haider, P. Deminskyi, M. Yilmaz, K. Elmabruk, I. Yilmaz and N. Biyikli, Journal of Materials Chemistry C, 2018.

123. P. Motamedi, N. Dalili and K. Cadien, Journal of Materials Chemistry C, 2015, 3, 74287436.

124. H.-Y. Shih, M.-C. Lin, L.-Y. Chen and M.-J. Chen, Nanotechnology, 2014, 26, 014002.

125. J. Sumakeris, Z. Sitar, K. Ailey-Trent, K. More and R. Davis, Thin Solid Films, 1993, 225, 244-249.

126. Y. Kumagai, M. Mayumi, A. Koukitu and H. Seki, Applied Surface Science, 2000, 159, 427431.

127. M. Bartram, T. Michalske, J. Rogers Jr and R. Paine, Chemistry of Materials, 1993, 5, 14241430.

128. J. Jokinen, P. Haussalo, J. Keinonen, M. Ritala, D. Riihelä and M. Leskelä, Thin Solid Films, 1996, 289, 159-165.

129. R. L. Puurunen, A. Root, P. Sarv, M. M. Viitanen, H. H. Brongersma, M. Lindblad and A. O. I. Krause, Chemistry of Materials, 2002, 14, 720-729.

130. S. Banerjee, A. A. I. Aarnink, R. van de Kruijs, A. Y. Kovalgin and J. Schmitz, Physica Status Solidi (C), 2015, 12, 1036-1042.

131. H. Van Bui, F. B. Wiggers, A. Gupta, M. D. Nguyen, A. A. I. Aarnink, M. P. de Jong and A. Y. Kovalgin, Journal of Vacuum Science \& Technology A: Vacuum, Surfaces, and Films, 2015, 33, 01A111. 
132. K.-H. Kim, N.-W. Kwak and S. H. Lee, Electronic Materials Letters, 2009, 5, 83-86.

133. M. Asif Khan, J. Kuznia, R. Skogman, D. Olson, M. Mac Millan and W. Choyke, Applied Physics Letters, 1992, 61, 2539-2541.

134. J. Kidder Jr, J. Kuo, A. Ludviksson, T. Pearsall, J. Rogers Jr, J. M. Grant, L. R. Allen and S. T. Hsu, Journal of Vacuum Science \& Technology A: Vacuum, Surfaces, and Films, 1995, 13, 711-715.

135. H. Liu and J. Rogers Jr, Journal of Vacuum Science \& Technology A: Vacuum, Surfaces, and Films, 1999, 17, 325-331.

136. Y. J. Lee and S.-W. Kang, Thin Solid Films, 2004, 446, 227-231.

137. A. Y. Kovalgin, M. Yang, A. A. I. Aarnink and R. A. M. Wolters, U.S. Patent No. 15/615,489, 2018.

138. M. Yang, A. A. I. Aarnink, J. Schmitz and A. Y. Kovalgin, Thin Solid Films, 2018, 649, 1723.

139. A. J. Onnink, R. O. Apaydin, J. Schmitz and A. Y. Kovalgin, EuroCVD 22-Baltic ALD 16 Conf., Luxembourg, June 2019.

140. A. J. Onnink, J. Schmitz and A. Y. Kovalgin, Thin Solid Films, 2019, 674, 22-32.

141. S. Banerjee, R. van der Velde, M. Yang, J. Schmitz and A. Y. Kovalgin, International Conference of Microelectronic Test Structures (ICMTS), Grenoble, March 2017.

142. R. L. Puurunen, Journal of Applied Physics, 2005, 97, 121301.

143. V. Miikkulainen, M. Leskelä, M. Ritala and R. L. Puurunen, Journal of Applied Physics, 2013, 113, 021301.

144. S. Sioncke, L. Nyns, Ts.Ivanov, D. Lin, J. Franco, A. Vais, M. Ameen, A. Delabie, Q. Xie, J.W. Maes, F. Tang, M.Givens, S. Van Elshocht, F.Holsteyns, K.Barla, N. Collaert, A. Thean, S. De Gendt and M. Heyns, ECS Transactions, 2014, 64 (9), 133-144.

145. G. D. Wilk, M. Verghese, P. J. Chen and J. W. Maes, ECS Transactions, 2012, 50 (4), 207-210. 


\section{Appendix 1: Monitoring the wafer temperature with in-situ SE}

Using the temperature-dependence of optical constants (i.e., refractive index $(n)$ and optical constants $(k)$ ) of a silicon wafer, it is possible to monitor the wafer temperature by insitu $\mathrm{SE}^{\ddagger}$. In ALD processes, in-situ estimation of the wafer temperature is important if the temperature sensor is not located at the site of the process (due to a risk of its contamination by the reactive gases) but at a distance from the chuck. Especially in some large-volume reactors, the temperature monitored by the sensor (thermocouple) can deviate as much as 100 ${ }^{\circ} \mathrm{C}$ from the chuck temperature, because of heat losses through convective flows.

Since ALD is a surface-governed chemical process, when the temperature window for self-limiting reactions is narrow, identifying the correct chuck temperature becomes a priority. Fig. A 1 a shows the $n$ and $k$ of $\mathrm{Si}$ at room temperature and at $400{ }^{\circ} \mathrm{C}$ (as reported in J. A. Woollam CompleteEASE software database). Fig. A 1 b shows the as-obtained difference between the SE reading (i.e., wafer temperature) and the thermocouple (TC) reading for the Cluster reactor. The close match between the two readings is attributed to the proximity of the TC to the chuck and to the small volume of the reactor which minimizes heat losses.
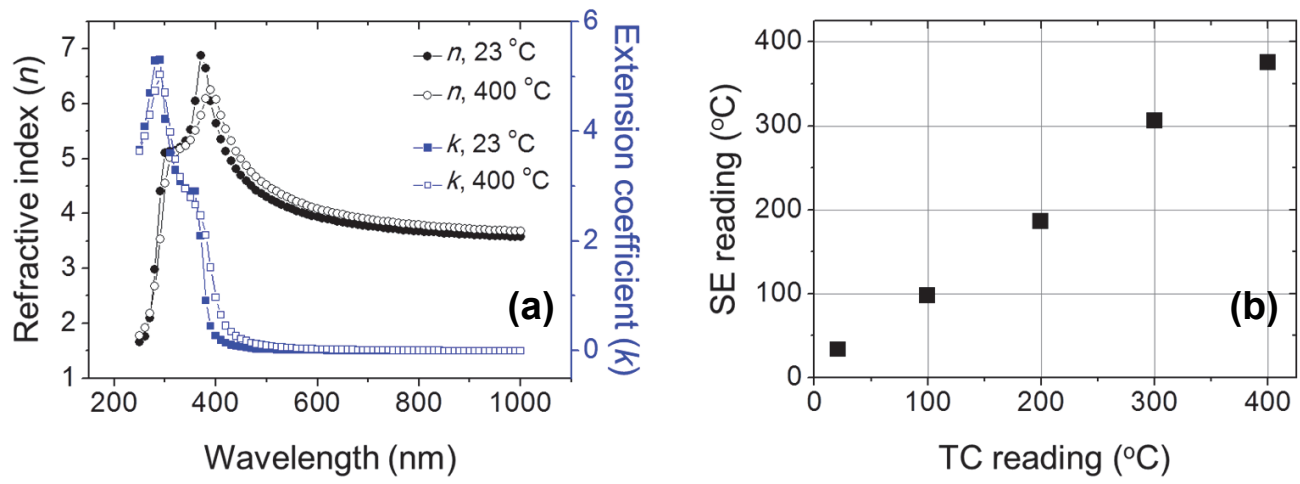

Fig. A 1 (a) Optical constants of $\mathrm{Si}$ at room temperature and at $400{ }^{\circ} \mathrm{C}$. (b) Variation of SE and TC readings for the Cluster ALD reactor.

* In place of $\mathrm{Si}$, a thermally-grown $\mathrm{SiO}_{2}$ wafer can also be used. In that case, it is necessary to pre-determine the oxide thickness. 


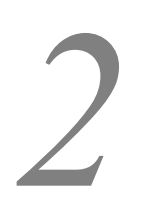

\section{Plasma-enhanced ALD}

\section{(PEALD) of polycrystalline AlN and $G a N^{*}$}

* This chapter comprises two sections: PEALD of AlN and PEALD of GaN.

The former section is based on:

S. Banerjee, A. A. I. Aarnink, R. vd Kruijs, A. Y. Kovalgin and J. Schmitz, physica status solidi (c), $2015,12(7), 1036-1042$.

The latter section is partly published in:

G. Gupta, S. Banerjee, S. Dutta, A. A. I. Aarnink, J. Schmitz, A. Y. Kovalgin and R. J. E. Hueting, Journal of Applied Physics, 2018, 124(8), 084503. 


\subsection{Introduction}

In this chapter, we explore plasma enhanced atomic layer deposition (PEALD) of polycrystalline $(\mathrm{Al}) \mathrm{GaN}$ layers, in a remote-plasma commercial Picosun R-200 reactor. The precursors for AlN are trimethylaluminium (TMA) and ammonia $\left(\mathrm{NH}_{3}\right)$, and for $\mathrm{GaN}$ are trimethylgallium (TMG) and $\mathrm{NH}_{3}$. The motivation of using plasma is to enhance the reactivity of the $\mathrm{NH}_{3}$ precursor, as well as tune the material properties of (Al) GaN layers by varying the plasma parameters. Furthermore, whereas both thermal and PEALD of AlN using these precursors has been reported ${ }^{1-3}$, thermal ALD of GaN using the same precursors has been hardly realized prior to this project; only PEALD has been reported for $\mathrm{GaN}^{4-5}$. Therefore, considering the possible impracticality of GaN ALD by a purely thermal process, the PEALD route is followed. This chapter presents the growth and detailed material characterization of $(\mathrm{Al}) \mathrm{GaN}$ layers. Improving the crystallinity of the layers is a major focus, as this can facilitate specific device applications, such as thin film transistors (TFT) and light emitting diodes (LED) ${ }^{6}$. PEALD films have been reported to have a higher crystallinity than the thermally-deposited counterparts ${ }^{7-8}$.

Section 2.1.1 presents a brief overview of PEALD. Section 2.2 addresses the PEALD of AlN, and Section 2.3 describes the PEALD of GaN. Specifically, Sections 2.2.1 - 2.2.2 describe the deposition conditions and the so-called 'ALD window' determination of AlN. Section 2.2.3 presents the characterization of AlN by spectroscopic ellipsometry (SE), X-ray photoelectron spectroscopy (XPS), Rutherford backscattering spectroscopy (RBS) and $\mathrm{X}$-ray diffraction (XRD), to reveal the optical properties, composition and crystallinity of AlN. Section 2.2.4 describes the optimization of the crystallinity of AlN films, using plasma power, plasma pre-treatment and pre-deposition of a seed layer. Section 2.3.1 presents the characterization of GaN layers by XPS and Fourier transform infrared spectroscopy (FTIR) for compositional and bonding environment analyses, XRD for crystallinity, and scanning \& transmission electron microscopy (SEM, TEM) for the layer morphology. Section 2.3.2 shows how changing the plasma composition - i.e., increasing the partial pressure of ammonia in the plasma - affects the polycrystallinity and optical properties of the GaN layers. Section 2.3.3 verifies the same effect for AlN layers. Section 2.4 concludes the chapter.

This chapter contains four appendices. Appendix 2.1 and 2.2 describe the X-ray photoelectron spectroscopy (XPS) and low energy ion scattering (LEIS) characterization of $\mathrm{GaN}$, Appendix 2.3 discusses the SE-based optical modelling procedure for $\mathrm{GaN}$, and Appendix 2.4 presents the study of plasma by optical emission spectroscopy (OES), to explain the crystallinity changes to the (Al)GaN layers with varying plasma composition. 


\subsubsection{PEALD: A brief overview}

In thin film microelectronic processes, a low deposition temperature is preferred in many applications. For example, in devices employing compound semiconductors (GaN, $\mathrm{GaAs}, \mathrm{CdTe}$, etc.), there is an upper-limit to the deposition temperature in order to prevent the preferential evaporation of the more-volatile element ${ }^{9}$. In other materials like amorphous hydrogenated-Si, high temperatures may lead to the loss of the bonded hydrogen, which degrades the semiconducting properties ${ }^{9}$. Further, for devices fabricated on doped Si substrates, high temperatures enhance the dopant diffusion and risk the mechanical stability, if the substrate-size is large ${ }^{9}$. The use of low-melting-point substrates such as polymers also dictate a temperature upper-limit ${ }^{10}$.

Additional agents of excitation in the deposition, such as the assistance of radicals and/or ions, reduce the temperature by activating one or more chemical pathways that are otherwise restricted thermodynamically. A plasma, generated from one or more precursors is a common tool for producing radicals. Owing to the significant knowledge of plasma-based processing in traditional deposition methods (e.g., sputtering, CVD), PEALD has remarkably progressed $^{11}$, enabling the deposition of a wide array of materials since its first report ${ }^{12}$. In PEALD, the plasma may be either continuously ignited, or only during a specific precursor pulse at every ALD cycle.

The plasma consists of several species such as radicals, charged particles (ions, electrons), photons and undissociated molecules, all or some of which contributing to the film formation. During delivery of the species to the substrate, they may get depleted by recombination and adsorption. Making use of this, two principle reactor designs exist: directplasma and remote-plasma reactors ${ }^{13}$. In direct-plasma reactors, the substrate is immersed in the plasma; the chuck acts as one of the plates of the capacitor forming the plasma. Therefore, the film receives a heavy flux of the plasma species. On the contrary, in remoteplasma reactors, the plasma is formed at a distance (e.g., a meter) from the substrate. The delivery of the species occurs mainly by diffusion, and the flux of charged particles can be optionally controlled by applying a substrate-bias ${ }^{14}$. Remote-plasma is useful when the film is prone to damage by the species flux. Moreover, it provides freedom to change the substrate condition (e.g., temperature) with minimal changes to the plasma chemistry. Similarly, changes to the plasma composition (e.g., variation of gas flows) can be performed without their direct (thermal) effect on the film.

The plasma composition has a strong influence on the film stoichiometry and properties, and can be analysed with optical emission spectroscopy (OES). In this technique, the fluorescence of the excited plasma species, as they return to lower energy levels, is measured $^{13}$. Based on the OES, the plasma composition can be optimized. A PEALD reactor, which is equipped with in-situ OES, can provide valuable information about the growth 
mechanism and thus the film properties ${ }^{15}$. The advantage of using plasma in ALD can be best described by Profijt et al., "plasmas can deliver a high, diverse but selective reactivity to a surface without heat, and can therefore access a parameter space in materials processing, which is not easily accessible with strictly chemical methods"13.

\subsection{PEALD of polycrystalline AIN layers}

\subsubsection{Deposition conditions}

The PEALD of AlN was performed in Picosun R-200 remote-plasma commercial ALD reactor from TMA and $\mathrm{NH}_{3}$ precursors. The precursors were delivered using carrier gases: $\mathrm{N}_{2}$ for TMA and Ar for $\mathrm{NH}_{3}$. The plasma consisted of a mixture of $\mathrm{NH}_{3}$ and Ar. A plasma power of $2 \mathrm{~kW}$, process temperature of $350{ }^{\circ} \mathrm{C}$, and reactor pressure of 1 mbar were maintained during the experiments, unless otherwise mentioned.

4-inch $\mathrm{Si}(111)$ wafers were used as substrates. Prior to ALD, they were cleaned from organic and metallic contaminants using $99 \%$ - and $69 \%$-by-vol. $\mathrm{HNO}_{3}$, respectively. The native oxide on Si was removed by dipping in HF (1 \%). In-situ SE (Woollam M-2000; spectral range $245-1800 \mathrm{~nm}$ ) was used to monitor the wafer temperature during heating up, prior to ALD, and during ALD, to monitor the AlN thickness and the growth per cycle (GPC) in real-time. With an optimized-for-ALD recipe (Section 2.2.2), the GPC was $0.09 \mathrm{~nm} /$ cycle.

\subsubsection{ALD window determination}

The ALD window for PEALD of AIN films was identified in terms of the precursor pulse durations and temperature, monitoring the variation of GPC with changes induced in these parameters (Fig. 2.1 a-c).

Fig. 2.1 a shows the change of GPC with the $\mathrm{NH}_{3}$ pulse duration, the latter varied between 1 and $30 \mathrm{~s}$. A TMA pulse of $0.1 \mathrm{~s}$ was used for these experiments. The GPC saturates between 4 and $8 \mathrm{~s}$ of the $\mathrm{NH}_{3}$ pulse. Below $4 \mathrm{~s}$, it drops sharply, indicating possibly-incomplete surface reactions. Beyond $8 \mathrm{~s}$, the GPC increases steadily. The reason can be the onset of gas-phase reactions (i.e., CVD mode) and subsequent deposition due to incomplete $\mathrm{NH}_{3}$ removal.

Fig. 2.1 b shows the variation of GPC with the TMA pulse duration, between 0.1 and 2 s. The $\mathrm{NH}_{3}$ pulse duration was $8 \mathrm{~s}$ for all experiments. The GPC saturates approximately beyond $0.3 \mathrm{~s}$, indicating self-limiting surface reactions. 
Fig. 2.1 c shows the variation of the GPC with temperature, the latter varied between 175 and $450{ }^{\circ} \mathrm{C}$. The TMA and $\mathrm{NH}_{3}$ pulse durations were $0.1 \mathrm{~s}$ and $8 \mathrm{~s}$, respectively. The GPC saturated in a narrow temperature range between 300 and $350{ }^{\circ} \mathrm{C}$, whereas a steady decrease was observed below $300^{\circ} \mathrm{C}$. In a separate experiment, the value of the GPC without using plasma was tested; there, it was negligible at $300{ }^{\circ} \mathrm{C}$. Therefore, the still-high GPC values at or below $300{ }^{\circ} \mathrm{C}$ in PEALD is due to the effect of the plasma-generated species. On the contrary, beyond $350{ }^{\circ} \mathrm{C}$ it shows a steady increase. This possibly occurs from the dissociation of TMA, leading to a loss of self-limiting reactions ${ }^{16}$.
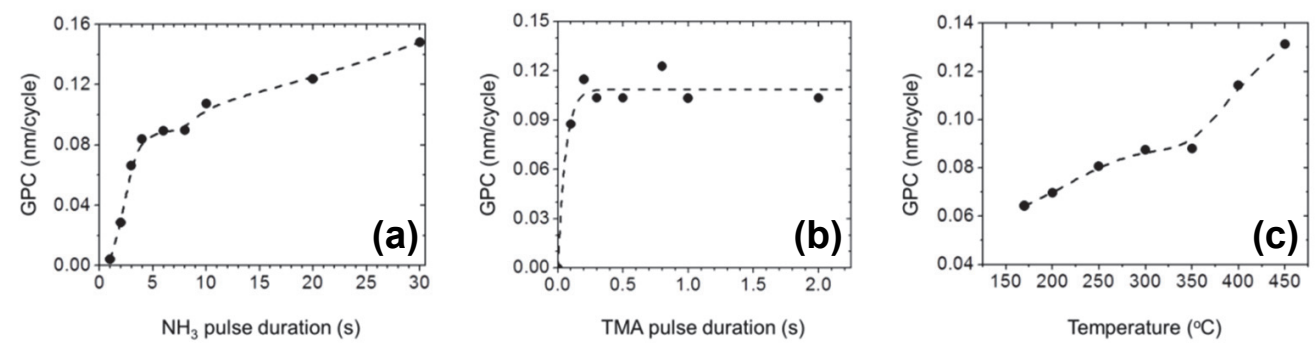

Fig. 2.1 Determination of the ALD window for PEALD AIN films by monitoring the variation of GPC with (a) $\mathrm{NH}_{3}$ pulse duration, (b) TMG pulse duration, and (c) Temperature.

True to ALD, in all above experiments, the GPC was less sensitive to the variations in the process parameters, signifying an ALD window where closest-to-self-limiting AlN growth presumably occurred. However, in each experiment, the values of the other parameters were maintained constant. Therefore, to test the validity of the so-obtained ALD window, the precursor durations were adjusted simultaneously and the resulting GPC was recorded (Table 2.1). The GPC varied only slightly $(0.10 \pm 0.02 \mathrm{~nm} /$ cycle $)$ for all combinations of the precursor durations. The increase from $0.07 \mathrm{~nm} /$ cycle for $0.1 \mathrm{~s} \mathrm{TMA}$ and $4 \mathrm{~s} \mathrm{NH}_{3}$ pulse (expt. 1) to $0.12 \mathrm{~nm} /$ cycle for $1 \mathrm{~s}$ TMA and $8 \mathrm{~s} \mathrm{NH}_{3}$ pulses (expt. 5) reflects under-saturated doses in the first case, since saturation was observed from $0.3 \mathrm{~s}$ TMA pulse. On the contrary, when long precursor pulses are applied (expt. 5), the GPC tends to be high. Expt. 3 is in the middle of the ALD window and consequently yields a median GPC value.

Table 2.1: Variation of GPC for different precursor durations in the ALD window.

\begin{tabular}{|c|c|c|c|}
\hline Experiment\# & TMA pulse (s) & $\mathbf{N H}_{3}$ pulse (s) & GPC (nm/cycle) \\
\hline 1 & 0.1 & 4 & 0.07 \\
\hline 2 & 0.1 & 8 & 0.09 \\
\hline 3 & 0.5 & 6 & 0.09 \\
\hline 4 & 1 & 4 & 0.08 \\
\hline 5 & 1 & 8 & 0.12 \\
\hline
\end{tabular}




\subsubsection{In-situ and ex-situ characterization}

2.2.3.1 Spectroscopic ellipsometry and optical modelling: In order to use SE for monitoring the AlN growth, a suitable optical model was constructed. Due to its wide bandgap ( $6.2 \mathrm{eV}$, corresponding to $200 \mathrm{~nm}$ ), the material should ideally be transparent over the entire spectral range of the SE. However, a sharp transition from transparent to absorbing wavelengths is hardly expected due to the presence of defects, impurities, grain boundaries etc., and the absorption decreases gradually towards the higher wavelengths, below the optical bandgap.

For AlN, the Cauchy optical model (Eqn. 2.1) was used to determine its thickness and refractive index ${ }^{17}$. This model is only functional at the wavelengths where the material is transparent. However, fitting the thickness and the three Cauchy parameters (Eqn. 2.1) all at the same time tends to decrease the fit accuracy. In a dispersion-free wavelength region, $B$ and $C$ are zero. This region was identified for AlN to be $>750 \mathrm{~nm}$, and thereafter, the thickness and parameter $A$ were fitted. A low mean squared error (MSE) between the acquired and fitted optical data, and a low correlation between the two fit parameters implied the accuracy of the model.

$$
\begin{array}{lll}
\text { Eqn. 2.1 } & n(\lambda)=A+\frac{B}{\lambda^{2}}+\frac{C}{\lambda^{4}}+\ldots & \begin{array}{l}
A, B \text { and } C \text { are Cauchy parameters } \\
\lambda \text { is the optical wavelength } \\
E \text { is the optical energy }
\end{array} \\
\text { Eqn. 2.2 } & k(\lambda)=K \frac{\lambda}{4 \pi} e^{E / E_{U}} & \begin{array}{l}
E_{U} \text { is the Urbach energy } \\
K \text { is the proportionality constant }
\end{array}
\end{array}
$$

The thickness evolution of AIN during ALD is shown in Fig. 2.2 a. The step-wise growth reflects the pulsed nature of the deposition (although it is not possible to identify the exact instants of the precursors in this experiment). The value of the dispersion-free refractive index $(A)$ was evaluated as 1.94 , comparable to literature-reported values for polycrystalline AlN films: $1.97^{18}, 1.90^{19}, 1.88^{2}$.

For determining the AlN optical constants (i.e., refractive index $(n)$ and extinction coefficient $(k)$ ) over the entire wavelength range, an Urbach tail relation ${ }^{20}$ (Eqn. 2.2) was introduced to the Cauchy optical model to describe the sub-bandgap absorptions. The absorptions manifest as the extinction coefficient. The optical constants' dependency on the wavelength is shown in Fig. 2.2 b, c. 

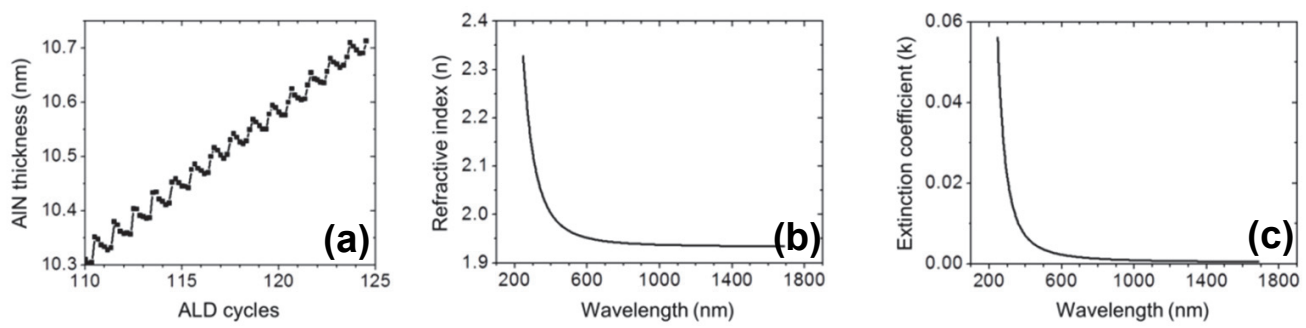

Fig. 2.2 (a) Real-time growth of PEALD AIN film, monitored by in-situ SE. (b) Dependency of refractive index $(n)$ and (c) extinction coefficient $(k)$ on wavelength.

After deposition, the thickness uniformity was mapped by ex-situ SE (Fig. 2.3 a). The scan shows low $(<3 \%)$ non-uniformity, i.e., as expected for ALD. The SE-obtained thicknesses were verified with high resolution scanning electron microscope (HR-SEM) images (an example is shown in Fig. 2.3 b). From atomic force microscopy (AFM) measurements (not shown), the film had a low surface roughness (2.4 nm RMS).
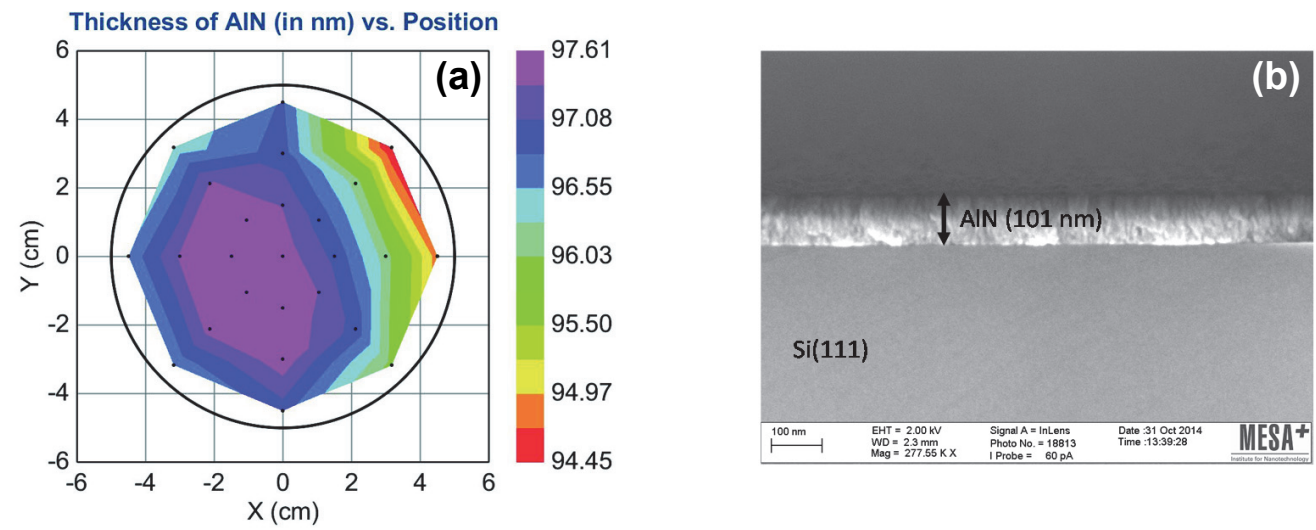

Fig. 2.3 (a) Thickness variation of PEALD AlN on a 4-inch Si substrate, mapped by ex-situ SE. (b) HR-SEM image of the layer.

2.2.3.2 X-ray photoelectron spectroscopy: To obtain the layer composition through its thickness, sputter-depth-profiled XPS was performed. Fig. 2.4 reveals a near-stoichiometric AlN layer, with average Al- and N-contents of 46 and 53 at. \%, respectively. These values remain almost constant throughout the film thickness. Low impurity levels in the form of oxygen and carbon were recorded, both $<2$ at. \%. The extremely low carbon reflects the efficient removal of the $-\mathrm{CH}_{3}$ groups of TMA by the plasma-generated radicals of $\mathrm{NH}_{3}$. $\mathrm{The}$ 
use of high-purity gases and purifiers enable the preparation of AlN films with negligible oxygen content. The increase in oxygen towards the surface is attributed to post-oxidation of the layer ${ }^{21}$ during its transfer to the XPS setup.

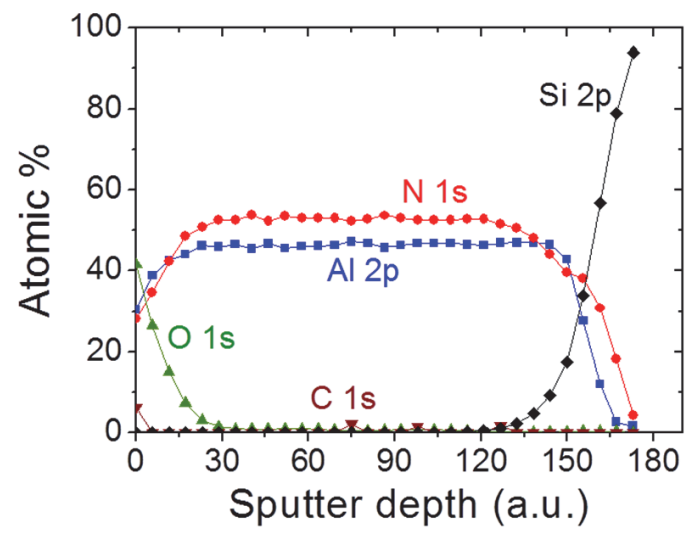

Fig. 2.4 Sputter depth profiled XPS composition of PEALD AlN film.

2.2.3.3 Rutherford backscattering spectroscopy: The layer was additionally analysed by RBS in the channelling configuration using a $2 \mathrm{MeV} \mathrm{He}^{+}$beam. Fig. 2.5 shows the depth profiled composition of the layer. A slightly higher N-content $(\sim 51$ at. \%) than Al-content $(\sim 43$ at. $\%)$ is observed again.

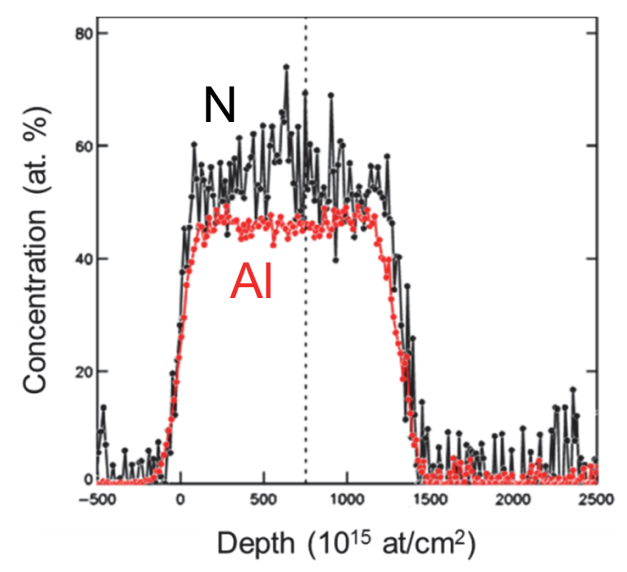

Fig. 2.5 Sputter depth profiled RBS composition of the film. 
2.2.3.4 X-ray diffraction by $\theta$-20, Grazing incidence and $\omega$-scans: AlN is reported to exist in hexagonal (wurtzitic) or cubic (zinc-blende) crystalline forms ${ }^{22}$. To comprehensively study the crystallinity and its possible orientations in the presumably polycrystalline layer, XRD analyses in $\theta$-2 $\theta$, grazing-incidence, and $\omega$-scan geometries were performed, using a Malvern Panalytical X'Pert powder diffractometer. The $\theta-2 \theta$ scan (Fig. 2.6 a) shows a small, broad peak centred at $35.8^{\circ}$. This corresponds to the formation of a small amount of vertically-oriented (002) planes of wurtzitic AlN (COD No. 9008860). However, only the planes parallel to the substrate are detected in the $\theta-2 \theta$ configuration. Tilted planes, that may be abundant in a polycrystalline sample, can be observed from grazing incidence X-ray diffraction (GIXRD) ${ }^{23}$.
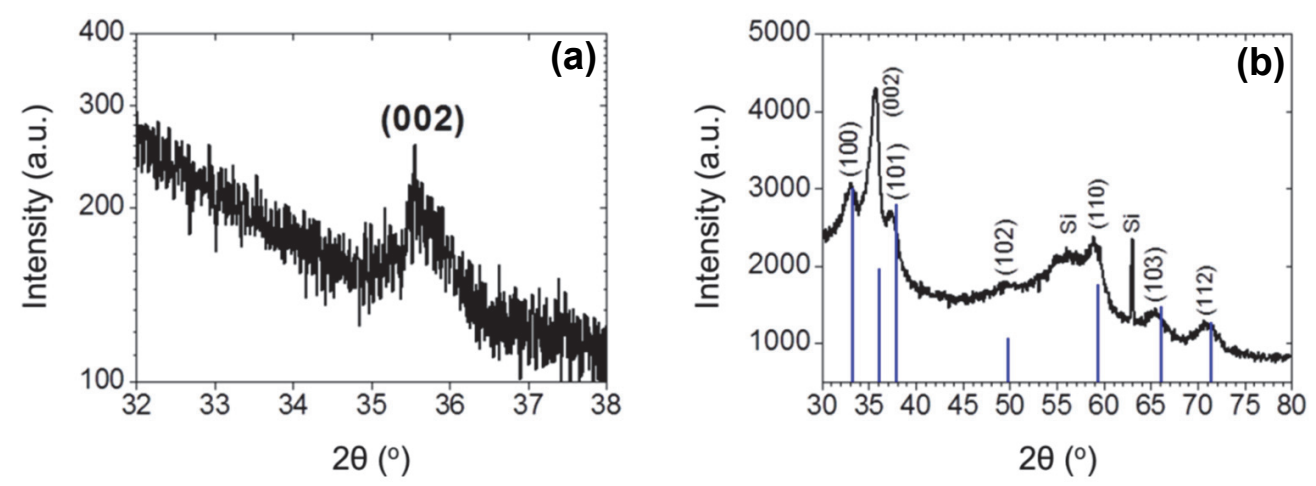

Fig. 2.6 (a) $\theta-2 \theta$ and (b) GIXRD scan of PEALD AIN. The bars in (b) represent the reported peak positions of wurtzitic AlN, and their Miller indices are shown.

The GIXRD scan (Fig. 2.6 b) was obtained with an angle of incidence $(\omega)$ of $1^{\mathrm{o}}$; the latter was low enough to illuminate the sample surface adequately, but was higher than the critical angle of the film, determined to be $0.2^{\circ}$ from X-ray reflectivity measurements. In Fig. 2.6 b, the appearance of several peaks, and their positions matching with that reported for wurtzitic AlN (COD No. 9008860) (as shown by the bars), confirm the polycrystalline wurtzitic nature of our PEALD AlN sample. Further, as in the $\theta-2 \theta$ scan, the (002) diffraction peak is similarly dominant in GIXRD. With the $\omega$ of $1^{\circ}$, these planes are tilted at $16.9^{\circ}$ to the vertical axis. The average grain size of the (002) planes was estimated to be $11 \mathrm{~nm}$. This was done using the Malvern Panalytical HighScore Plus software, after assuming a pseudo-Voigt peak profile and an intrinsic peak-broadening with $2 \theta$, described by the Caglioti relation ${ }^{24}$.

To partially answer whether similar (002)-plane dominance was present at various other tilts, $\omega$-scans were performed, comparing the intensity of the (002) peak with that of the (100) and (101) peaks (Fig. 2.7). In these measurements, the angle between the source 
and the detector (i.e., $2 \theta$ ) was fixed at $33.1^{\circ}, 35.8^{\circ}$ and $37.8^{\circ}$, respectively, corresponding to those obtained from GIXRD. The $\omega$-scan from an off-peak-position $\left(2 \theta\right.$ of $\left.53^{\circ}\right)$ was also recorded as reference. The gradual decay of the signals with an increasing $\omega$ is due to the decreasing volume of the film under illumination. Up to an $\omega$ of $\sim 10^{\circ}$, a clear dominance of the (002) plane, under various tilts, is observed. Due to the rapidly-attenuating intensity at higher $\omega$ values, and also limited by the diffractometer geometry, the (002) dominance could not be verified for other tilts.

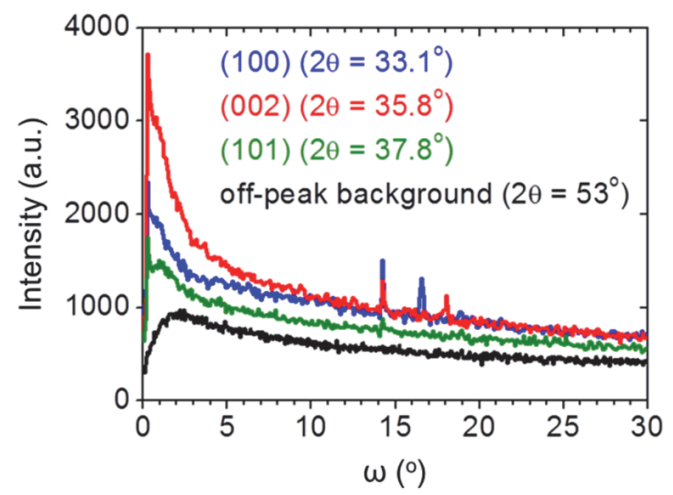

Fig. $2.7 \omega$-scans of the (100), (002) and (101) wurtzitic planes of AlN, measured at $2 \theta$ of $33.1^{\circ}, 35.8^{\circ}$ and $37.8^{\circ}$, respectively.

\subsubsection{Crystallinity optimization of PEALD AIN layers}

ALD is hardly the preferred technique for monocrystalline films, especially beyond a certain thickness when poly-crystals begin to form. This is because, ALD is a surfacegoverned chemical process, and several factors may be present that intervene the epitaxial lateral growth, resulting in poly-crystals. Some of the factors are (i) presence of impurity molecules such as oxidants, (ii) steric hindrance from the chemisorbed precursor blocking chemisorption to its neighbouring sites, (iii) incomplete (or absence of) reactions due to orientation, etc ${ }^{25}$. When an additional activation agent (e.g., plasma) is used, the energetic species can improve the film crystallinity to some extent, by for example enhancing surface migration and/or removal of by-products. Therefore, the effect of plasma on the polycrystallinity of AlN was tested, as will be discussed next.

2.2.4.1 Effect of plasma power: A higher plasma power provides a greater energy to the substrate in the form of a higher species flux, thereby enhancing the species' surface migration mobility, and presumably altering / enhancing the surface reactions. An enhanced surface mobility facilitates the formation of a layer with better crystallinity ${ }^{18}$. Besides, plasma power effects the concentration of the different species. In this light, depositions 
were performed under 1.5 and $2.5 \mathrm{~kW}$ plasma, and the AlN polycrystallinity was observed by GIXRD (Fig. 2.8). Raising the power slightly enhances the formation of the (002) over the (100) plane, whereas that of the (101) plane remains unchanged. Perhaps due to the remote-plasma setup, these changes are rather modest.

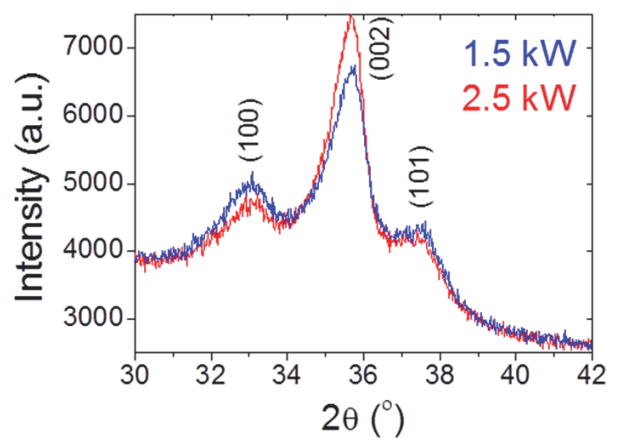

Fig. 2.8 GIXRD of $\sim 100 \mathrm{~nm}$ AlN films deposited under 1.5 and $2.5 \mathrm{~kW}$ plasma power.
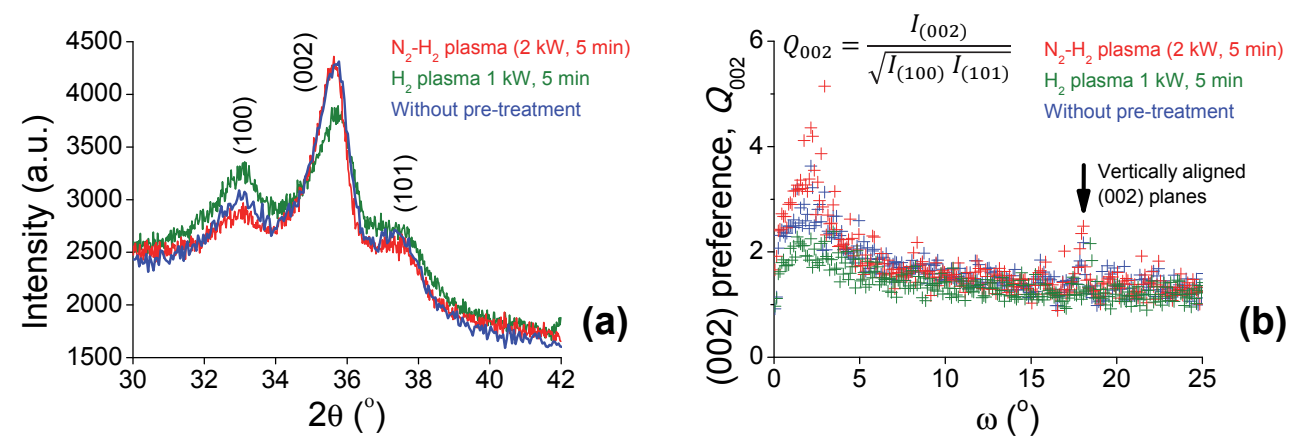

Fig. 2.9 (a) GIXRD of AIN deposited with and without plasma pre-treatment. Two plasma compositions were used, as indicated. The films were $\sim 100 \mathrm{~nm}$ thick. (b) Variation of $Q_{002}$ with $\omega$, representing the preferred formation of (002) planes at various tilts.

2.2.4.2 Effect of in-situ substrate pre-treatment: The crystallinity of the substrate may influence the film crystallinity in ALD, as in other deposition techniques. Moreover, furnishing the substrate with specific terminations that participate in the film-forming reactions, may enable smooth transition from the substrate to the film, thereby influencing the film crystallinity. In-situ plasma-based pre-treatment of the substrate can be a means to achieve this. 
Three plasma compositions were used for surface pre-treatment: $\mathrm{NH}_{3}-, \mathrm{N}_{2}-\mathrm{H}_{2-}$, and $\mathrm{H}_{2}$-plasmas. $\mathrm{Si}(111)$ substrates were used in all cases. The composition, the pre-treatment duration, and the plasma power had varying effects on the AlN polycrystallinity (Fig. 2.9 a). Pre-treatment with $\mathrm{H}_{2}$-plasma $(1 \mathrm{~kW}, 5 \mathrm{~min}$ ) caused slightly higher growth of (100) over (002) planes (in green), whereas $\mathrm{N}_{2}-\mathrm{H}_{2}$ plasma pre-treatment ( $2 \mathrm{~kW}, 5 \mathrm{~min}$ ) caused the opposite effect (in red). Pre-treatment with $\mathrm{NH}_{3}$ plasma $(2 \mathrm{~kW}, 5 \mathrm{~min})$ also favoured the formation of the (100) plane (not shown). All these changes were quite minor.

For the three samples discussed above, $\omega$-scans of the (100), (002) and (101) planes were performed up to an $\omega$ of $25^{\circ}$. To show the crystallinity changes quantitatively, a metric $\left(Q_{002}\right)$ was used, formulated as $Q_{002}=I_{002} / \sqrt{I_{100} I_{101}}$; where $I$ is the intensity of the corresponding (i.e., (002), etc.) planes, obtained from the $\omega$-scans. The variation of $Q_{002}$ with $\omega$ is shown in Fig. 2.9 b. The $\mathrm{N}_{2}-\mathrm{H}_{2}$ plasma pre-treatment resulted in the highest (002) formation at an $\omega$ of $2.95^{\circ}$, which corresponds to $14.9^{\circ}$ to the vertical axis. On the contrary, $\mathrm{H}_{2}$-plasma pre-treatment favoured the formation of the (100) over the (002) plane, at all $\omega$ values.

2.2.4.3 Effect of rapid thermal annealing of in-situ deposited AIN seed layer: Whereas plasma pre-treatment of the $\operatorname{Si}(111)$ substrate yielded only minor changes to the polycrystallinity of the AIN layers, the pre-deposition of a seed AIN layer of $7 \mathrm{~nm}$, with the same ALD recipe, caused bigger change. The seed layer was subjected to rapid thermal annealing in $\mathrm{H}_{2}$ ambient. Fig. $\mathbf{2 . 1 0}$ compares the polycrystallinity of AIN films deposited with (in blue) and without (in red) the seed-layer. Clearly, the annealed seed-layer favours the formation of the (100) over (002) planes. We speculate that annealing caused the seed-layer to (re-)crystallize in the (100) orientation, providing a template for the latter film growth.

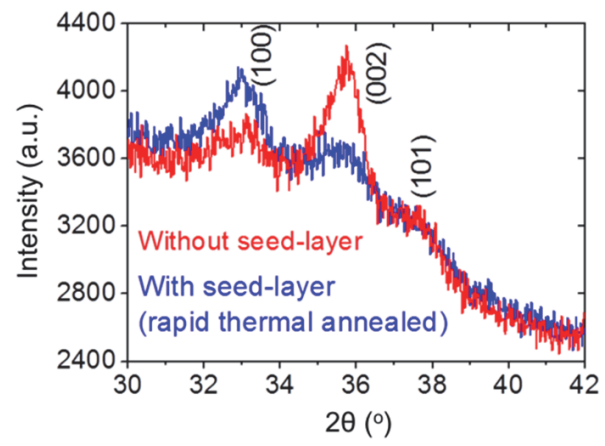

Fig. 2.10 GIXRD of AlN layers prepared with and without a rapid-thermal-annealed seed layer. 


\subsection{PEALD of polycrystalline GaN layers}

Polycrystalline GaN layers were deposited in the Picosun reactor by PEALD, using TMG and $\mathrm{NH}_{3}$ precursors. The depositions were performed at $400{ }^{\circ} \mathrm{C}$, at a pressure of 1 mbar. The ALD recipe was $0.1 \mathrm{~s}$ TMG / $6 \mathrm{~s} \mathrm{~N}_{2}$ purge / $11 \mathrm{~s} \mathrm{NH}_{3} / 10 \mathrm{~s}$ Ar purge. The plasma power was $2 \mathrm{~kW}$.

\subsubsection{Ex-situ characterization}

2.3.1.1 X-ray photoelectron spectroscopy: The chemical bonding and composition of the layers were analysed by sputter depth profiled XPS. Fig. 2.11 a and b show the photoelectron $\mathrm{Ga}_{2} \mathrm{p}_{3 / 2}$ and $\mathrm{N}$ 1s peaks, respectively, acquired from the bulk of the layer. The former can be well-fitted with one Gaussian-Lorentzian band. This suggests (tentatively) a single bonding environment of $\mathrm{Ga}$. The binding energy (BE) at $1117.6 \mathrm{eV}$ is in excellent agreement with that of $\mathrm{N}-\mathrm{Ga}$ bonds, reported at $1117.8 \mathrm{eV}^{26}$ or $1117.9 \mathrm{eV}^{27}$. No indication of elemental $\mathrm{Ga}$, i.e., a band with $\mathrm{Ga}-\mathrm{Ga}$ bonds at $1116.5 \mathrm{eV}^{28}$, was observed.
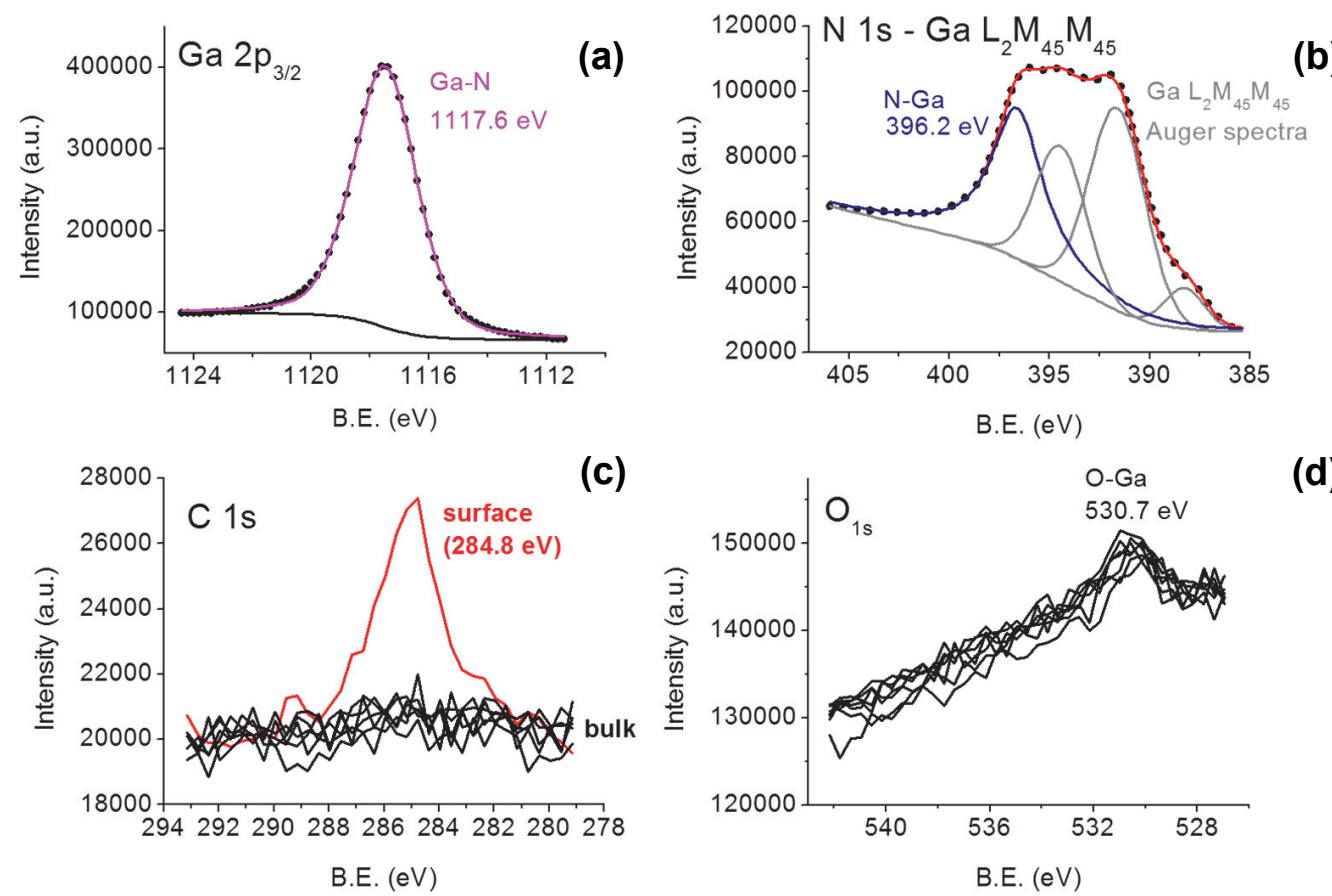

(d)

Fig. 2.11 XPS spectra of PEALD GaN films, showing (a) Ga 2p $p_{3 / 2}$ photoelectron spectrum, (b) $\mathrm{N} 1 \mathrm{~s}$ photoelectron - Ga $\mathrm{L}_{2} \mathrm{M}_{45} \mathrm{M}_{45}$ Auger triplet, (c) $\mathrm{C} 1 \mathrm{~s}$ spectra on the surface (red) and inside the film (black), and (d) $\mathrm{O} 1 \mathrm{~s}$ spectra inside the film. 
Fig. 2.11 b reveals the deconvolution of the $\mathrm{N}$ 1s photoelectron spectrum (in blue) from the $\mathrm{Ga} \mathrm{L}_{2} \mathrm{M}_{45} \mathrm{M}_{45}$ Auger triplet spectra (in grey) ${ }^{29}$ (see Appendix 2.1). The BE of the $\mathrm{N}$ 1s spectrum $(396.2 \mathrm{eV})$ corresponds to the literature-reported $\mathrm{N}-\mathrm{Ga} \mathrm{BE}$ of $396.4 \mathrm{eV}^{29}$ or $396.2 \mathrm{eV}^{30}$. The Auger spectra overlaps with this BE range because the XPS is done with an $\mathrm{Al} \mathrm{k} \alpha \mathrm{X}$-ray source of $1486.6 \mathrm{eV}$ energy ${ }^{31}$.

Low impurity levels (i.e., $\mathrm{C}$ and $\mathrm{O}$ ) were detected in the layer (Fig. 2.11 c, d). Fig. 2.11 c shows the $C 1$ s photoelectron spectra at the surface (in red) and at different thicknesses (in black). The surface spectrum arises from adventitious carbon contamination, with a $\mathrm{BE}$ of $284.8 \mathrm{eV}^{32}$. Hardly any carbon is detected inside the GaN layer.

Fig. 2.11 d shows the $\mathrm{O} 1 \mathrm{~s}$ spectra at different thicknesses in the layer. It is approximately constant in magnitude and is larger than the C-content. The peak at $530.7 \mathrm{eV}$ is lower than $\mathrm{O}-\mathrm{Ga} \mathrm{BE}$ in stoichiometric $\mathrm{Ga}_{2} \mathrm{O}_{3}$ layers reported at $531.8 \mathrm{eV}^{33}$ and $531.9 \mathrm{eV}^{34}$. Because of the lower electronegativity of $\mathrm{N}$ (3.04) compared to $\mathrm{O}(3.44)^{35}$, a down-shift of the $\mathrm{O}-\mathrm{Ga} \mathrm{BE}$ is expected when $\mathrm{O}$ is bonded to the $\mathrm{GaN}$ network in Ga-oxynitride, instead of forming segregated clusters of $\mathrm{Ga}_{2} \mathrm{O}_{3}$. A similar down-shift of the $\mathrm{O} 1 \mathrm{~s} \mathrm{BE}$ from $531.9 \mathrm{eV}$ to $530.7 \mathrm{eV}$ is reported from an oxidized GaN surface to the bulk of the film; the bulk containing lesser amounts of oxygen ${ }^{26}$. In other examples, a range of $\mathrm{O}-\mathrm{Ga} \mathrm{BE}$ values reported in GaN films depends on their O-content: $531.7 \mathrm{eV}^{36}$ or $530.4 \mathrm{eV}^{29}$. The overall oxygen content in our PEALD GaN film is rather low, approximately 7 at. \% as estimated from the depth-profiled composition (Fig. 2.12). This explains fitting of the $\mathrm{Ga} 2 \mathrm{p}_{3 / 2}$ spectrum by a single band (Fig. 2.11 a). The strong Ga-N band submerged the weak Ga-O band.

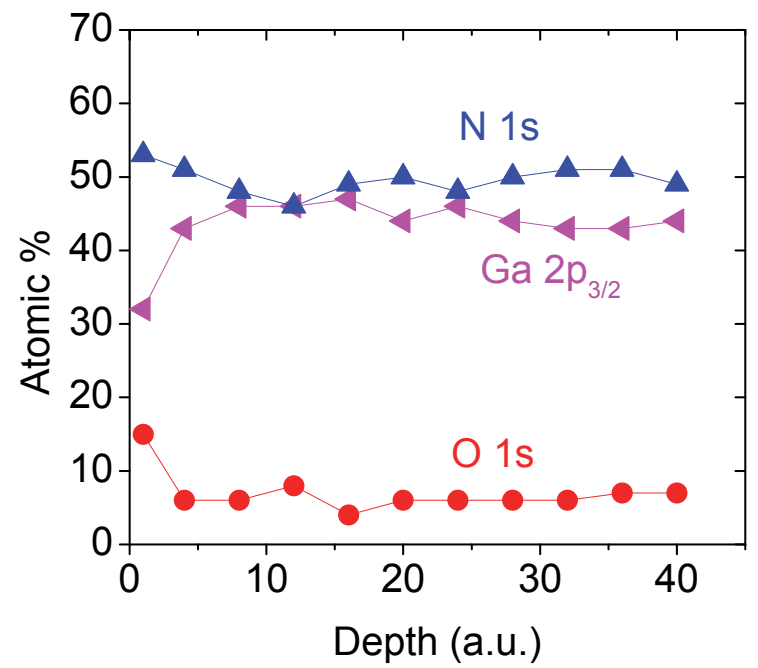

Fig. 2.12 Sputter depth profiled XPS composition of PEALD GaN film. 
The depth-profiled composition (Fig. 2.12) reveals that the layer is N-rich, just as the AlN was found to be (recall Fig. 2.4). This could possibly occur due to the the high flux of N-containing species from the plasma taking part in the surface reactions; however, detailed investigation was not done. The N-rich composition of GaN was also qualitatively observed from low energy ion scattering (LEIS) measurements (Appendix 2.2).

2.3.1.2 Fourier transform infrared spectroscopy: Fig. 2.13 shows the FTIR transmission spectrum of the PEALD GaN layer. The major absorption at $551 \mathrm{~cm}^{-1}$ is located in the vicinity of the Ga-N stretching vibration of wurtzitic $\mathrm{GaN}$, reported at $545 \mathrm{~cm}^{-1(37)}$. The overshoot between 2000 and $2500 \mathrm{~cm}^{-1}$ arises from differences in $\mathrm{CO}_{2}$ levels (inside the spectrometer) between measuring the background and the actual sample. No other significant absorption is observed.

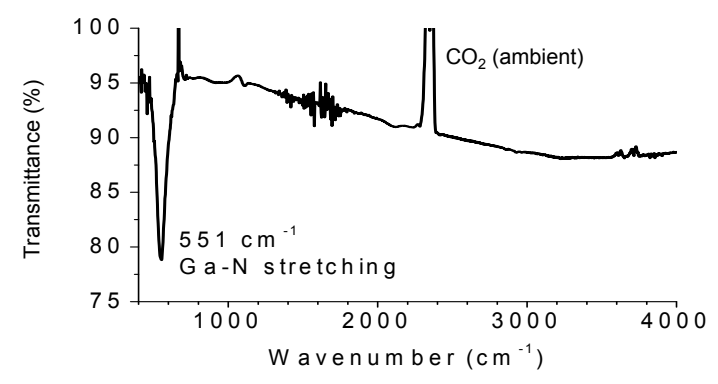

Fig. 2.13 FTIR transmission spectrum of PEALD GaN.

2.3.1.3 Scanning and Transmission electron microscopy: HR-SEM InLens and energy selected backscatter (ESB) images of the layer deposited on Si are shown in Fig. 2.14 a and b, respectively.
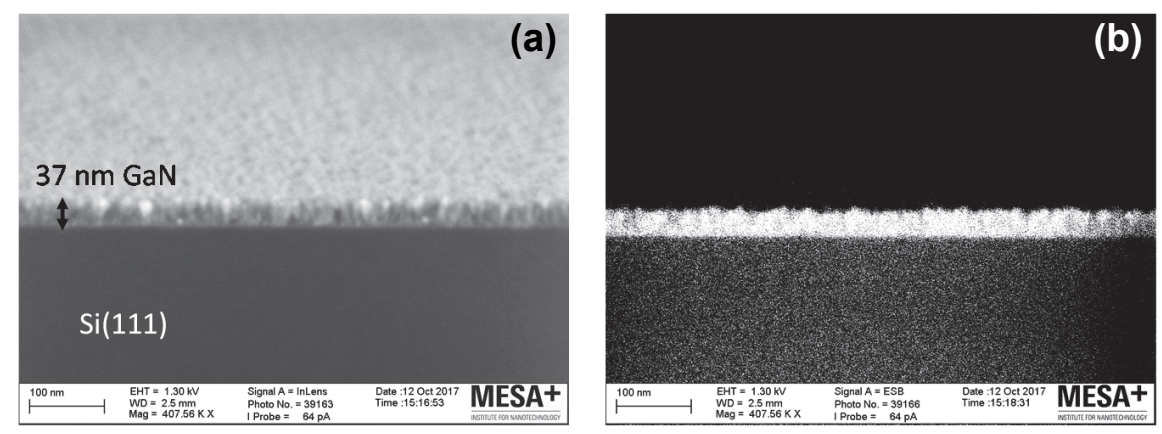

Fig. 2.14 (a) HR-SEM (b) HR-ESB images of PEALD GaN on Si(111) substrate. 
TEM analysis (Fig. 2.15 a, b and d) revealed the columnar growth at several regions inside a polycrystalline matrix, with the column-width increasing from the substrate towards the surface in a few cases. The grain sizes varied between 3 and $9 \mathrm{~nm}$. Fast Fourier transform (FFT) at several regions in the polycrystalline layer revealed $d$-spacing values $(2.6 \pm 0.04 \AA)$ predominantly associated with the (002) planes of wurtzitic GaN (COD No. 9011658), as well as presence of other planes such as (100) and (101) in smaller amounts. Fig. 2.15 c shows a polycrystalline region, which is the magnified view of the boxed region in b. Its FFT image, with diffused diffraction pattern due to the polycrystallinity, is shown in the inset.
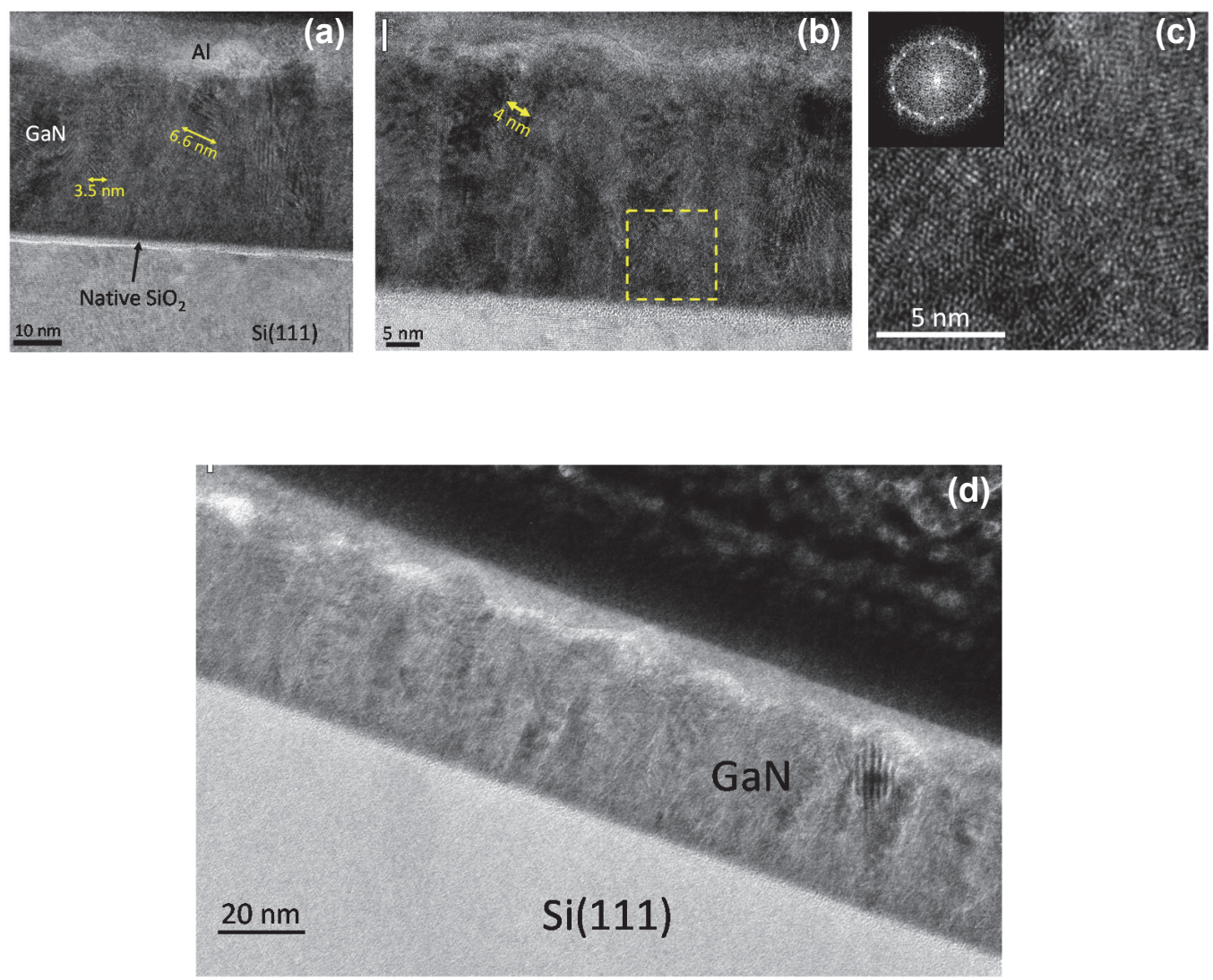

Fig. 2.15 (a, b) TEM images of the PEALD GaN layer showing crystalline columns at certain locations, with their sizes indicated. (c) Magnified image of the boxed region of $\mathbf{b}$, revealing a polycrystalline region; the inset shows the FFT image of this region. (d) Zoomed-out view of the GaN layer showing the columnar growth. 
2.3.1.4 Grazing incidence X-ray diffraction: The polycrystallinity was further revealed from GIXRD (Fig. 2.16). The wurtzitic structure was verified from the similar $2 \theta$ values of the reported peak positions (COD No. 9011658), as shown by the blue bars. The strong (002) peak re-confirms the abundance of this plane in the layer. The average grain size of (002) planes was estimated to be $\sim 8 \mathrm{~nm}$ from GIXRD.

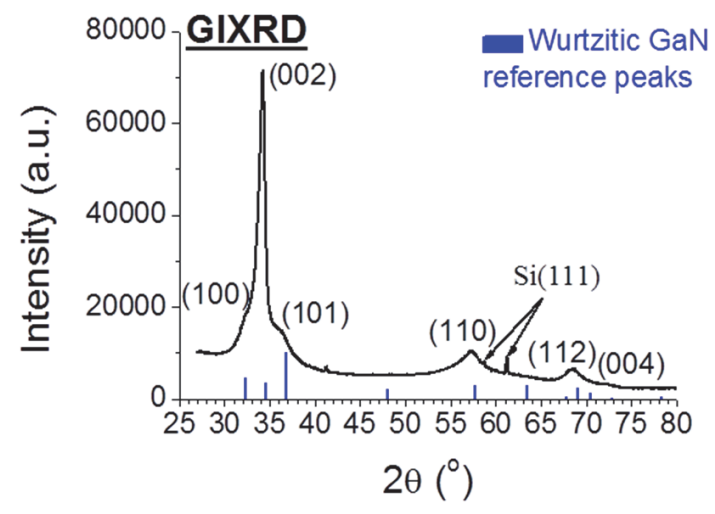

Fig. 2.16 GIXRD scan of PEALD GaN deposited on $\mathrm{Si}(111)$ substrate, revealing a wurtzitic structure. The literature-reported peak positions of wurtztic $\mathrm{GaN}$ are shown by blue bars.

\subsubsection{Effect of plasma composition on the polycrystallinity of GaN}

The polycrystallinity of PEALD GaN films was optimized by varying the partial pressure of $\mathrm{NH}_{3}\left(P_{\mathrm{NH} 3}\right)$ (equivalently, the mole fraction $\left(x_{\mathrm{NH} 3}\right)$ ) in the $\mathrm{NH}_{3}-\mathrm{Ar}$ plasma* ${ }^{*}$ This was achieved by adjusting the $\mathrm{NH}_{3}$ flow rate $\left(Q_{\mathrm{NH} 3}\right)$, while maintaining a constant flow rate of Ar. Three GaN samples of similar thicknesses $(35-38 \mathrm{~nm})$ were deposited. Table 2.2 lists the deposition parameters and the material properties of these samples. Samples 2 and 3 have almost identical material properties; which is as expected since they were deposited under similar $P_{\mathrm{NH} 3}$. On the contrary, sample 1, deposited under high $P_{\mathrm{NH} 3}$, shows major differences in the refractive index, optical bandgap, and the grain size of the (002) crystallites (to note: all the characterization results of Section 2.3.1 were from this sample).

\footnotetext{
* To note: The $P_{\mathrm{NH} 3}$ and $x_{\mathrm{NH} 3}$ reported are measured in the gas line, before entering the reactor. These values are therefore indicative and do not correspond to the true partial pressure (composition) of the plasma region.
} 
Table 2.2: Process parameters and material properties of PEALD GaN layers deposited under different $\mathrm{NH}_{3}$ partial pressures. The Ar flow rate was maintained at $250 \mathrm{sccm}$ and the $\mathrm{NH}_{3}$ flow rate was varied between 2 to $112 \mathrm{sccm}$, which resulted in an increase in the $\mathrm{NH}_{3}$ mole fraction from 0.05 to 0.60 . $(\mu$ and $\sigma$ represent the mean and standard deviation of thickness values obtained from wafer-mapping. The low $\sigma$ reflects a layer with high thickness uniformity.)

\begin{tabular}{|c|c|c|c|c|c|c|c|c|c|c|}
\hline \multicolumn{4}{|c|}{ Process parameters } & \multicolumn{7}{|c|}{ Material properties } \\
\hline \multirow[t]{2}{*}{ Sample } & \multirow{2}{*}{$\begin{array}{c}Q_{\mathrm{NH} 3} \\
(\mathrm{sccm})\end{array}$} & \multirow{2}{*}{$\begin{array}{c}P_{\mathrm{NH} 3} \\
\text { (mbar) }\end{array}$} & \multirow[t]{2}{*}{$x_{\mathrm{NH} 3}$} & \multicolumn{2}{|c|}{$\begin{array}{c}\text { Thickness } \\
\text { (from SE) } \\
(\mathrm{nm})\end{array}$} & \multirow{2}{*}{$\begin{array}{c}n \\
\text { (at } 1000 \\
n m)\end{array}$} & \multirow{2}{*}{$\begin{array}{c}E_{\mathrm{g}} \\
(\mathrm{eV})\end{array}$} & \multicolumn{3}{|c|}{$\begin{array}{c}\text { Average grain size } \\
(\mathbf{n m})\end{array}$} \\
\hline & & & & $\boldsymbol{\mu}$ & $\sigma$ & & & $(100)$ & $(002)$ & (101) \\
\hline 1 & 112 & 13 & 0.60 & 37 & 1.0 & 2.25 & 3.42 & 2 & 8 & 2 \\
\hline 2 & 6 & 2 & 0.10 & 37 & 0.4 & 2.20 & 3.91 & 3 & 2 & 4 \\
\hline 3 & 2 & 1 & 0.05 & 35 & 0.4 & 2.20 & 3.91 & 2 & 2 & 2 \\
\hline
\end{tabular}
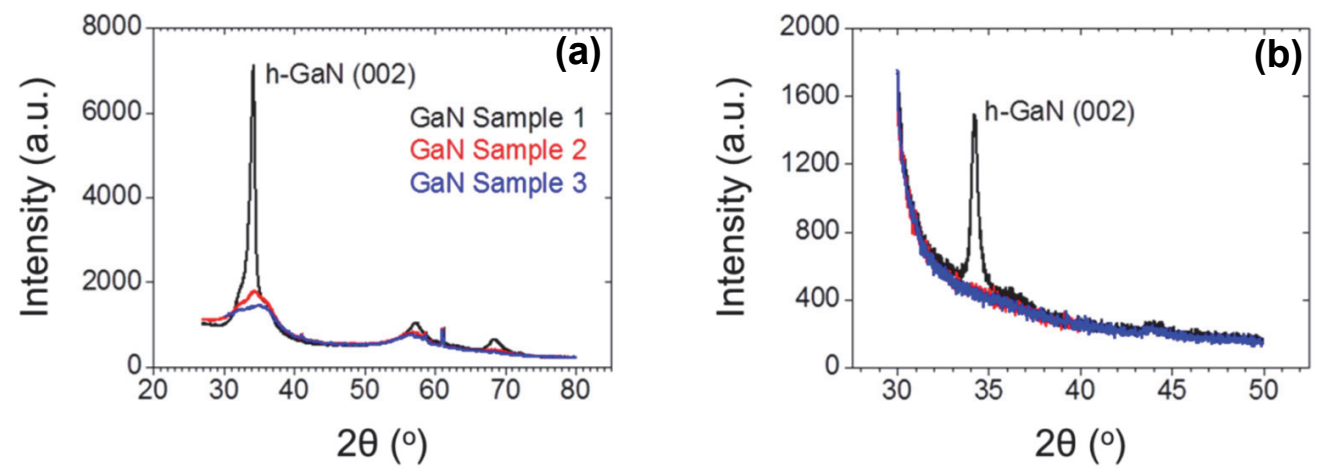

Fig. 2.17 (a) GIXRD, (b) $\theta-2 \theta$ XRD of PEALD GaN samples deposited under various $P_{\mathrm{NH} 3}$ values (Table 2.2).

The major change in the polycrystallinity of sample 1 is also revealed from GIXRD and $\theta-2 \theta$ scans (Fig. 2.17 a, b). The sample shows the highest crystalline order, compared to the other two. It is polycrystalline, wurtzitic, and shows strong diffraction peak of the (002) planes in both XRD scans. This reveals the abundance of (002) planes oriented in the tilted directions and parallel to the substrate. On contrary, samples 2 and 3 show an almost amorphous nature in both scans. 
Whereas the average grain sizes of the (100) and (101) planes in all three samples are equivalent (Table 2.2), that of the (002) plane increases with $P_{\mathrm{NH} 3}$, and consequently sample 1 has the largest average grain-size of $\sim 8 \mathrm{~nm}$.

The variation of polycrystallinity with $P_{\mathrm{NH} 3}$ is accompanied with consistent changes in the optical constants (Fig. 2.18 a, b) (Appendix 2.3 addresses the optical modelling procedure). Samples 2 and 3 have almost identical $n$ and $k$ at all wavelengths, whereas the $n$ of sample 1 is consistently higher, and its $k$ slightly lower than that of the others. The $n$ of all three samples however lie in the range of literature-reported values of PEALD GaN films: $2.15^{4}, 2.25^{38}$ and $2.24^{39}$ at $1000 \mathrm{~nm}$ wavelength.
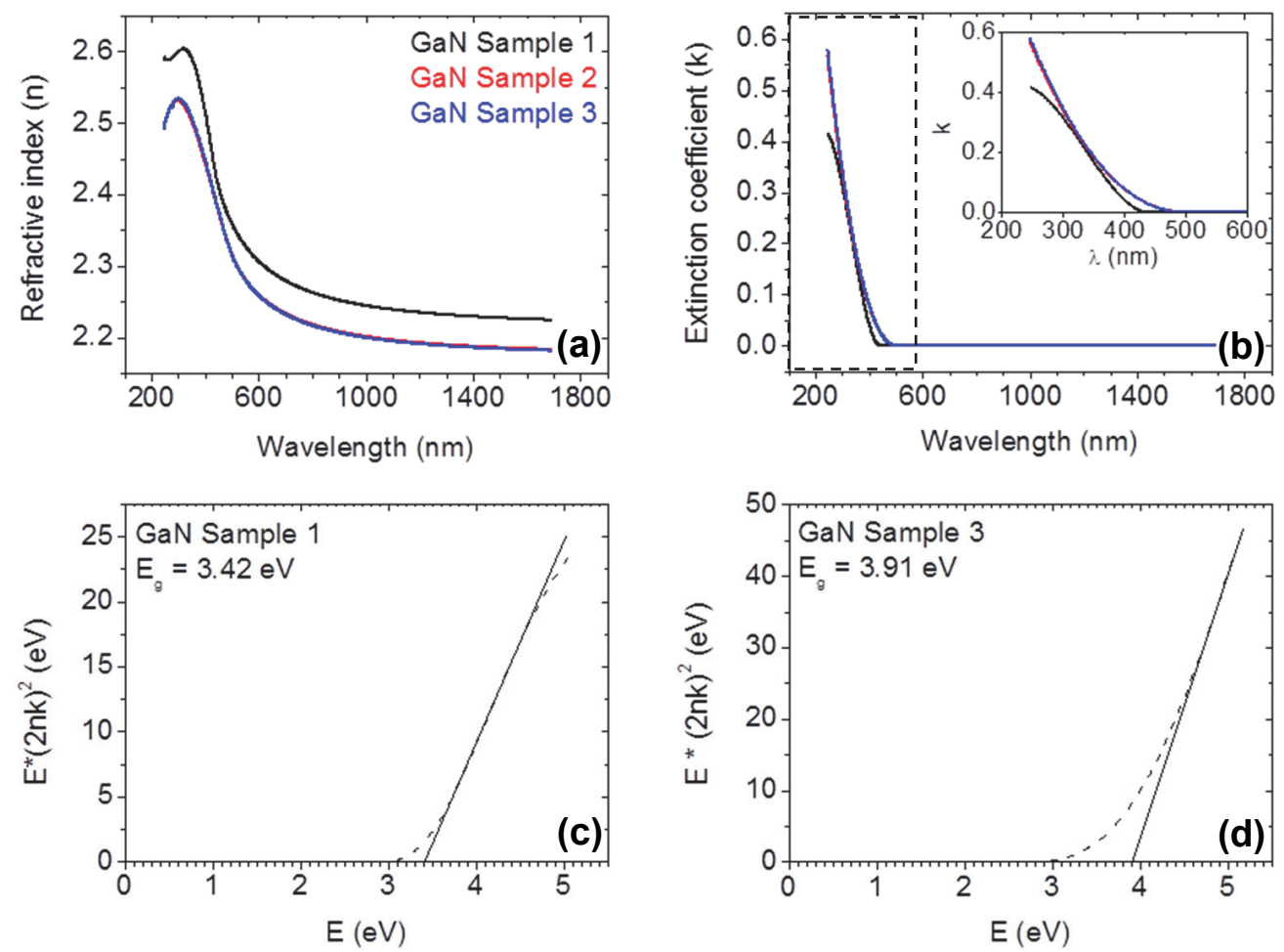

Fig. 2.18 Variation of (a) $n$ and (b) $k$ of PEALD GaN samples with wavelength. The inset zooms in the onset of the absorption. (c, d) Tauc plots and the estimated $E_{\mathrm{g}}$ of GaN samples 1 and 3, respectively. 
From $n$ and $k$, the so-called Tauc plots were constructed and the optical bandgaps $\left(E_{\mathrm{g}}\right)$ were determined ${ }^{\dagger}$. The Tauc plots of samples 1 and 3 are presented in Fig. $2.18 \mathbf{c}$ and d, respectively, and reveal $E_{\mathrm{g}}$ of 3.42 for sample 1 and $3.91 \mathrm{eV}$ for sample 3 . The $E_{\mathrm{g}}$ of sample 1 is in the range of that reported for poly-GaN films elsewhere ${ }^{38}$. Furthermore, the Tauc plots of samples 2 and 3 were almost identical due to their similar $n$ and $k$, and the $E_{\mathrm{g}}$ of both samples were $3.91 \mathrm{eV}$.

\subsubsection{Comparing the effect of plasma composition on the polycrystallinity of AIN}

The effect of the plasma composition on the polycrystallinity and optical properties was also investigated in PEALD AlN films. The $\mathrm{NH}_{3}-\mathrm{Ar}$ plasma was observed to change colour from violet to orange on decreasing $P_{\mathrm{NH} 3}$. Accordingly, three AlN samples were deposited: sample 1 using a strong violet-coloured plasma (corresponding to $P_{\mathrm{NH} 3}$ of 15 mbar or $x_{\mathrm{NH} 3}$ of 0.70$)$, sample 2 when the colour changed from violet to orange $\left(P_{\mathrm{NH} 3}=7\right.$ mbar; $\left.x_{\mathrm{NH} 3}=0.32\right)$, and sample 3 using a strong orange plasma $\left(P_{\mathrm{NH} 3}=2\right.$ mbar; $x_{\mathrm{NH} 3}=0.08$ ). For AlN, similar-to-GaN trends in material properties versus $P_{\mathrm{NH} 3}$ were obtained (Fig. 2.19 a and b). These observations verify that having a high $P_{\mathrm{NH} 3}$ in the $\mathrm{NH}_{3}-\mathrm{Ar}$ plasma is essential for obtaining polycrystalline (Al)GaN layers with higher $n$ and lower $k$ values. Appendix 2.4 addresses OES studies of the $\mathrm{NH}_{3}-\mathrm{Ar}$ plasmas used, and provides an explanation behind these changes.
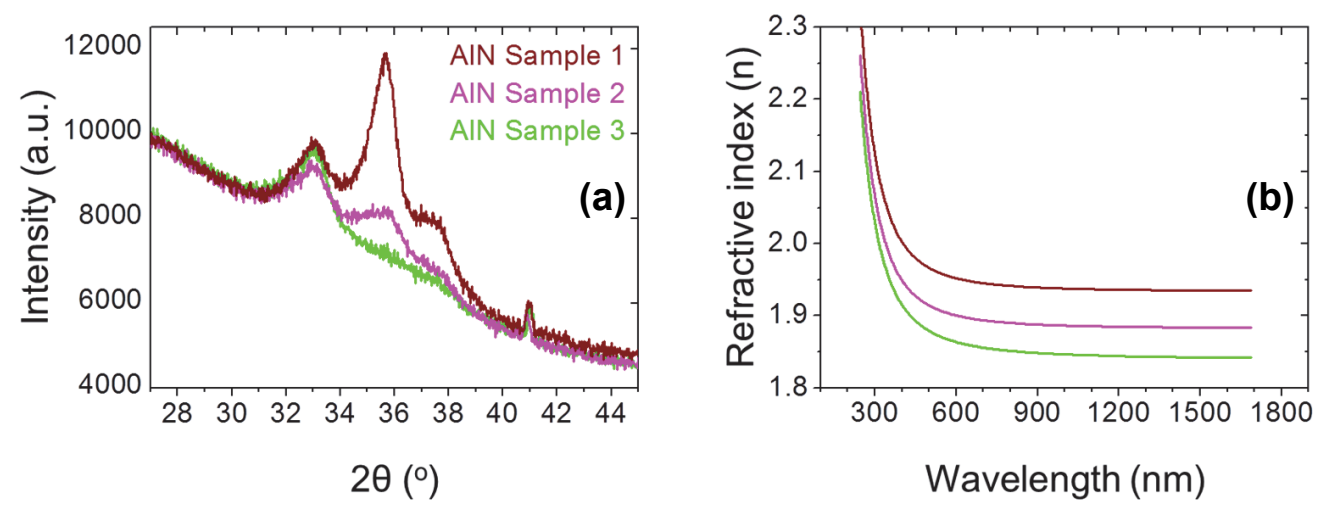

Fig. 2.19 Variation of (a) Crystallinity and (b) Refractive index variation of PEALD AlN layers with $P_{\mathrm{NH} 3}$.

\footnotetext{
${ }^{\dagger}$ A Tauc plot $^{54}$ is the plot of $E .(2 n k)^{1 / m}$ vs. $E$, where $E$ is the energy of the SE beam (in eV), and $m$ is a constant depending on the nature of the bandgap: 2.0 for direct and 0.5 for indirect semiconductors ${ }^{55}$. Extrapolating the linear region to the abscissa yields an estimate of the optical bandgap.
} 


\subsection{Conclusions}

This chapter introduced polycrystalline $(\mathrm{Al}) \mathrm{GaN}$ layers prepared by PEALD. The ALD window of AlN was identified by in-situ monitoring the influence of the precursor durations and temperature on GPC. Both AlN and GaN layers were characterized by a variety of techniques such as SE, XRD, FTIR, XPS, RBS, SEM and TEM. This revealed the optical properties, polycrystallinity, composition, bonding environment, and morphology of the layers.

Several plasma parameters were varied to influence the polycrystallinity of AlN layers. A higher plasma power favoured the formation of the wurtzitic (002) planes over the (100) and (101) planes. Similar effect was observed on treating the substrate $(\operatorname{Si}(111))$ with $\mathrm{N}_{2}-\mathrm{H}_{2}$ plasma prior to ALD. On the contrary, the deposition of an AlN seed layer, and its subsequent rapid thermal annealing before ALD, favoured the growth of (100) planes.

For GaN, the plasma composition had a strong effect on the polycrystallinity and optical constants. A high partial pressure (equivalently, the mole fraction) of $\mathrm{NH}_{3}$ in the $\mathrm{NH}_{3}-\mathrm{Ar}$ plasma resulted in strong preferential formation of the (002) planes, and consequently an increase of the grain size. This was accompanied by a higher refractive index and lower (i.e., close to stoichiometric GaN) optical bandgap. On the contrary, when deposited with a plasma of low $\mathrm{NH}_{3}$ partial pressure, the layers were almost amorphous and exhibited lower $n$, larger $E_{\mathrm{g}}$ and $k$. These effects were similarly observed for AlN, deposited under high and low $\mathrm{NH}_{3}$ partial pressures. Appendix 2.4 provides an explanation behind these observations in terms of OES measurements of such plasmas. 


\section{References}

1. H. Van Bui, M. Nguyen, F. B. Wiggers, A. A. I. Aarnink, M. P. de Jong and A. Y. Kovalgin, ECS Journal of Solid State Science and Technology, 2014, 3, P101-P106.

2. C. Ozgit, I. Donmez, M. Alevli and N. Biyikli, Thin Solid Films, 2012, 520, 2750-2755.

3. X. Liu, S. Ramanathan, E. Lee and T. E. Seidel, MRS Online Proceedings Library Archive, 2004, 811.

4. C. Ozgit-Akgun, E. Goldenberg, A. K. Okyay and N. Biyikli, Journal of Materials Chemistry C, 2014, 2, 2123-2136.

5. P. Pansila, K. Kanomata, M. Miura, B. Ahmmad, S. Kubota and F. Hirose, Applied Surface Science, 2015, 357, 1920-1927.

6. C. Liu, S. Liu, S. Huang and K. J. Chen, IEEE Electron Device Letters, 2013, 34, 1106-1108.

7. Y. Lee, S. Kim, J. Koo, I. Kim, J. Choi, H. Jeon and Y. Won, Journal of The Electrochemical Society, 2006, 153, G353-G357.

8. J. Musschoot, D. Deduytsche, H. Poelman, J. Haemers, R. Van Meirhaeghe, S. Van den Berghe and C. Detavernier, Journal of The Electrochemical Society, 2009, 156, P122-P126.

9. G. Lucovsky and D. Tsu, Journal of Vacuum Science \& Technology A: Vacuum, Surfaces, and Films, 1987, 5, 2231-2238.

10. J. Schmitz, Surface and Coatings Technology, 2018, 343, 83-88.

11. H. C. Knoops, T. Faraz, K. Arts and W. M. M. Kessels, Journal of Vacuum Science \& Technology A: Vacuum, Surfaces, and Films, 2019, 37, 030902.

12. M. De Keijser and C. Van Opdorp, Applied Physics Letters, 1991, 58, 1187-1189.

13. H. Profijt, S. Potts, M. Van de Sanden and W. Kessels, Journal of Vacuum Science \& Technology A: Vacuum, Surfaces, and Films, 2011, 29, 050801.

14. H. Profijt, M. Van de Sanden and W. Kessels, Journal of Vacuum Science \& Technology A: Vacuum, Surfaces, and Films, 2013, 31, 01A106.

15. D. Longrie, K. Devloo-Casier, D. Deduytsche, S. Van den Berghe, K. Driesen and C. Detavernier, ECS Journal of Solid State Science and Technology, 2012, 1, Q123-Q129.

16. T. M. Mayer, J. Rogers Jr and T. Michalske, Chemistry of Materials, 1991, 3, 641-646.

17. H. Van Bui, A. Aarnink, M. de Jong and A. Kovalgin, NEVAC blad, 2013, 51, 24-31.

18. H. Van Bui, F. B. Wiggers, A. Gupta, M. D. Nguyen, A. A. I. Aarnink, M. P. de Jong and A. Y. Kovalgin, Journal of Vacuum Science \& Technology A: Vacuum, Surfaces, and Films, 2015, 33, 01A111. 
19. P. Motamedi and K. Cadien, Journal of Crystal Growth, 2015, 421, 45-52.

20. O. Stenzel, The Physics of Thin Film Optical Spectra, Springer, 2005.

21. M. Leskelä and M. Ritala, Le Journal de Physique IV, 1995, 5, C5-937-C935-951.

22. S. Strite and H. Morkoç, Journal of Vacuum Science \& Technology B: Microelectronics and Nanometer Structures Processing, Measurement, and Phenomena, 1992, 10, 1237-1266.

23. M. Yasaka, The Rigaku Journal, 2010, 26, 2.

24. G. Caglioti, A. t. Paoletti and F. Ricci, Nuclear Instruments, 1958, 3, 223-228.

25. M. Bartram, T. Michalske, J. Rogers Jr and R. Paine, Chemistry of Materials, 1993, 5, 14241430 .

26. R. Carin, J. Deville and J. Werckmann, Surface and Interface Analysis, 1990, 16, 65-69.

27. Y. Jia, J. S. Wallace, E. Echeverria, J. A. Gardella Jr and U. Singisetti, Physica Status Solidi (B), 2017, 254, 1600681 .

28. J. L. Bourque, M. C. Biesinger and K. M. Baines, Dalton Transactions, 2016, 45, 7678-7696.

29. M. Alevli, A. Haider, S. Kizir, S. A. Leghari and N. Biyikli, Journal of Vacuum Science \& Technology A: Vacuum, Surfaces, and Films, 2016, 34, 01A137.

30. G. Moldovan, M. Roe, I. Harrison, M. Kappers, C. J. Humphreys and P. Brown, Philosophical Magazine, 2006, 86, 2315-2327.

31. B. Zhou, X. Li, T. Tansley, K. Butcher and M. Phillips, Journal of Crystal Growth, 1995, 151, 249-253.

32. T. L. Barr and S. Seal, Journal of Vacuum Science \& Technology A: Vacuum, Surfaces, and Films, 1995, 13, 1239-1246.

33. Y. Mizokawa, H. Iwasaki, R. Nishitani and S. Nakamura, Journal of Electron Spectroscopy and Related Phenomena, 1978, 14, 129-141.

34. I. Lopez, A. D. Utrilla, E. Nogales, B. Mendez, J. Piqueras, A. Peche, J. Ramírez-Castellanos and J. M. González-Calbet, The Journal of Physical Chemistry C, 2012, 116, 3935-3943.

35. A. Allred, Journal of Inorganic and Nuclear Chemistry, 1961, 17, 215-221.

36. S. Pal, R. Mahapatra, S. Ray, B. Chakraborty, S. Shivaprasad, S. Lahiri and D. Bose, Thin Solid Films, 2003, 425, 20-23.

37. B. Deb, A. Ganguly, S. Chaudhuri, B. Chakraborti and A. Pal, Materials Chemistry and Physics, 2002, 74, 282-288.

38. E. Goldenberg, C. Ozgit-Akgun, N. Biyikli and A. Kemal Okyay, Journal of Vacuum Science \& Technology A: Vacuum, Surfaces, and Films, 2014, 32, 031508. 
39. N. Gungor and M. Alevli, Journal of Vacuum Science \& Technology A: Vacuum, Surfaces, and Films, 2018, 36, 021514.

40. G. Jellison Jr and F. Modine, Applied Physics Letters, 1996, 69, 371-373.

41. G. Jellison Jr and F. Modine, Applied Physics Letters, 1996, 69, 2137-2137.

42. M. Alevli, N. Gungor, A. Haider, S. Kizir, S. A. Leghari and N. Biyikli, Journal of Vacuum Science \& Technology A: Vacuum, Surfaces, and Films, 2016, 34, $01 \mathrm{~A} 125$.

43. Software manual, J. A. Woollam Co., CompleteEASE, Version 5.19, 1999-2016.

44. D.-M. Rosu, E. Ortel, V.-D. Hodoroaba, R. Kraehnert and A. Hertwig, Applied Surface Science, 2017, 421, 487-493.

45. M. Hannemann, S. Hamann, I. Burlacov, K. Börner, H.-J. Spies and J. Röpcke, Surface and Coatings Technology, 2013, 235, 561-569.

46. N. Ihashi, K.-i. Itoh and O. Matsumoto, Plasma Chemistry and Plasma Processing, 1997, 17, 453-465.

47. D. B. Mawhinney, J. A. Glass Jr and J. T. Yates Jr, Journal of Vacuum Science \& Technology A: Vacuum, Surfaces, and Films, 1999, 17, 679-685.

48. S. Lucas, W. Partlow, W. Choyke and J. Yates Jr, Journal of Vacuum Science \& Technology A: Vacuum, Surfaces, and Films, 1994, 12, 3040-3047.

49. Y. S. Hiraoka and M. Mashita, Japanese Journal of Applied Physics, 1992, 31, 3703.

50. N. Preschilla A, S. Major, N. Kumar, I. Samajdar and R. Srinivasa, Applied Physics Letters, 2000, 77, 1861-1863.

51. M. J. Paterson, E. Goldys, H. Zuo and T. Tansley, Japanese Journal of Applied Physics, 1998, 37, 426.

52. R. Vispute, S. Choopun, R. Enck, A. Patel, V. Talyansky, R. Sharma, T. Venkatesan, W. Sarney, L. Salamancariba and S. Andronescu, Journal of Electronic Materials, 1999, 28, 275-286.

53. F. De Chelle, J. Berger, J. Ferraton and C. Ance, Thin Solid Films, 1987, 150, 181-188.

54. J. Tauc, R. Grigorovici and A. Vancu, Physica Status Solidi (B), 1966, 15, 627-637.

55. T. S. Moss, G. J. Burrell and B. Ellis, Semiconductor Opto-Electronics, ButterworthHeinemann, 2013.

56. S. Darwiche, M. Nikravech, S. Awamat, D. Morvan and J. Amouroux, Journal of Physics D: Applied Physics, 2007, 40, 1030.

57. L. Li, A. Nikiforov, N. Britun, R. Snyders and C. Leys, Spectrochimica Acta Part B: Atomic Spectroscopy, 2015, 107, 75-85. 
58. R. Henck, C. Fuchs and E. Fogarassy, Journal of Applied Physics, 1996, 79, 2259-2269.

59. W. C. Martin, V. Kaufman and A. Musgrove, Journal of Physical and Chemical Reference Data, 1993, 22, 1179-1212.

60. S. E. Alexandrov and A. Y. Kovalgin, Advanced Materials for Optics and Electronics, 1998, 8, 13-22.

61. K. Bayes and G. Kistiakowsky, The Journal of Chemical Physics, 1960, 32, 992-1000. 


\section{Appendix 2.1: XPS analysis of GaN}

The difficulty of analysing GaN films with XPS, using an Al k $\alpha$ monochromatic Xray source $(1486.6 \mathrm{eV})$ is that, the $\mathrm{N}$ 1s photoelectron spectrum is significantly overlapped by the $\mathrm{Ga}_{2} \mathrm{M}_{45} \mathrm{M}_{45}$ Auger spectra. The Auger spectra is composed of a triplet, i.e., a midband and two side-lobes. This makes accurately determining the area under the $\mathrm{N} 1 \mathrm{~s}$ spectrum rather difficult, and is prone to cause an over-estimation of the N-content in the $\mathrm{GaN}$ film ${ }^{29}$. For instance, during the analysis of a commercial (and assumed to be stoichiometric) $\mathrm{GaN}$ sample, assigning the entire area under the $\mathrm{N} 1 \mathrm{~s}-\mathrm{Ga} \mathrm{L}_{2} \mathrm{M}_{45} \mathrm{M}_{45}$ complex to nitrogen, resulted in a composition of $35 \% \mathrm{Ga}$ and $65 \% \mathrm{~N}$.

This problem is not encountered in other group III-Nitrides (e.g., AlN, BN) because their Auger spectra are separated from their N 1s peak positions. To demonstrate this, Fig. A 2.1 shows the Auger spectra from elemental $\mathrm{Ga}$ (in black) and the $\mathrm{N} 1 \mathrm{~s}$ photoelectron spectrum from BN (in red) (both spectra are from the database of PHI MultiPak XPS software). These spectra are compared to the overlapped $\mathrm{N} 1 \mathrm{~s}-\mathrm{Ga} \mathrm{L}_{2} \mathrm{M}_{45} \mathrm{M}_{45}$ complex in our GaN layer (in blue).

To de-convolute the $\mathrm{N}$ 1s photoelectron from the Auger spectra and determine the GaN composition, a possible approach is to de-convolute the triplet in the elemental Ga sample using Gaussian-Lorentzian bands, and then use the same fit parameters (i.e., relative peak positions and full width half maxima) for the GaN layer. When tried on the commercial sample, this approach yielded $48 \% \mathrm{Ga}$ and $52 \% \mathrm{~N}$, respectively. The deconvolution of PEALD and thermal ALD GaN samples is shown in Fig. 2.11 b and Chapter 5, Fig. 5.12 c, respectively. The Ga-content is determined from the area under the Ga2p $3 / 2$ peak in all cases.

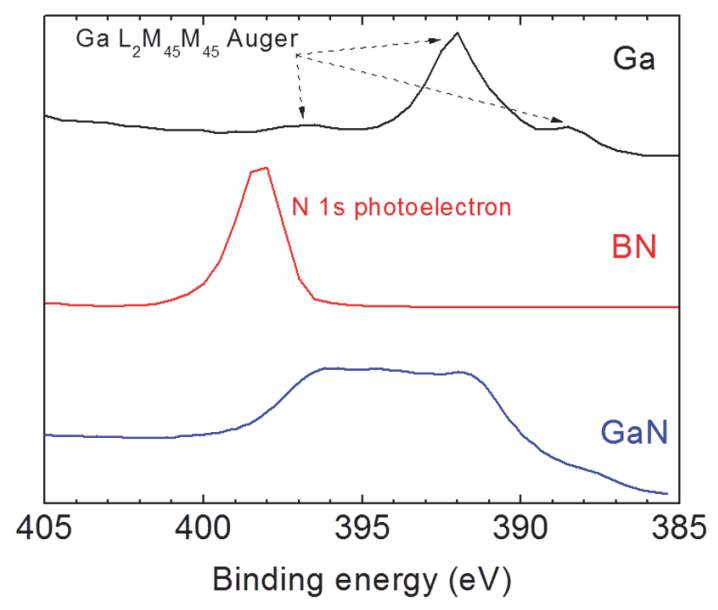

Fig. A 2.1 Ga $\mathrm{L}_{2} \mathrm{M}_{45} \mathrm{M}_{45}$ Auger spectra of elemental Ga (black), and $\mathrm{N}$ 1s photoelectron spectrum of $\mathrm{BN}$ (red) from database. The convoluted N 1s - Ga $\mathrm{L}_{2} \mathrm{M}_{45} \mathrm{M}_{45}$ complex in our GaN layer (blue). 


\section{Appendix 2.2: Low energy ion scattering (LEIS)}

The N-richness of PEALD GaN can be confirmed qualitatively when its sputter depth profiled LEIS analyses is compared to a commercial GaN reference sample. Fig. A 2.2 a, b shows the integrated elemental peak areas of the two samples. Compared to the reference, the Ga-to-N peak-integral ratio is smaller for PEALD GaN, suggesting the N-rich nature of the latter. Two assumptions were made for the comparison: (i) the relative peak intensities of the samples were independent of their bonding environment and crystallinity, and (ii) similar degree of preferential sputtering of nitrogen ${ }^{26}$ in the two samples.
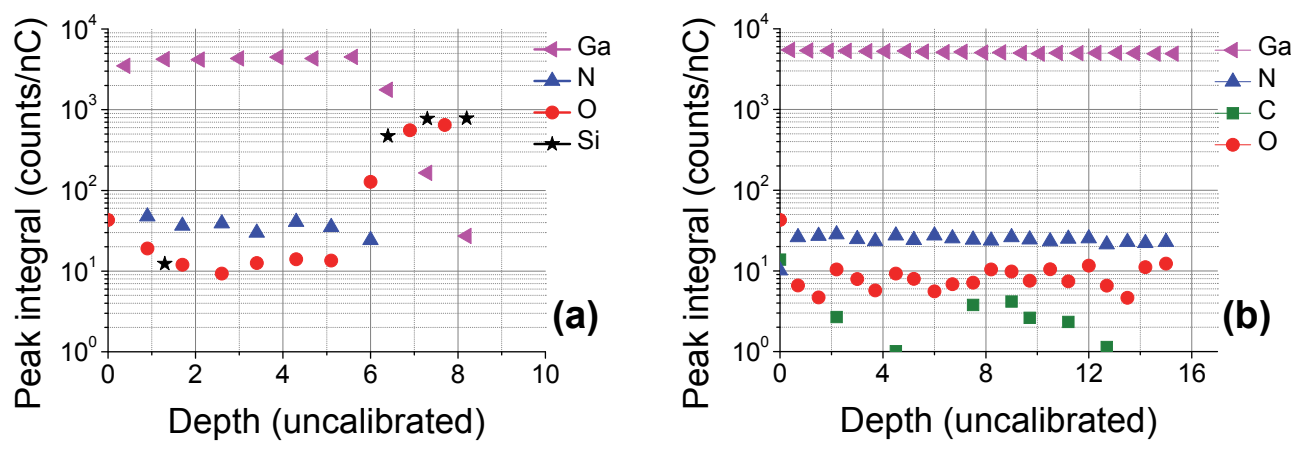

Fig. A 2.2 Depth-profiled LEIS composition of (a) PEALD GaN, and (b) a commercial GaN sample. 


\section{Appendix 2.3: Spectroscopic ellipsometry of PEALD GaN}

In-situ monitoring the growth of GaN layers was performed in a similar manner as that of AlN; i.e., fitting the thickness and the dispersion-free refractive index in the transparent wavelengths. However, the optical constants of GaN were determined using a Tauc-Lorentz model instead of Cauchy-Urbach model (the latter was used for AlN). The reason is the lower optical bandgap of $\mathrm{GaN}(3.4 \mathrm{eV})$ compared to AlN $(6.2 \mathrm{eV})$, which therefore, is situated within the ellipsometer spectral range. The presence of sub-bandgap optical transitions also contributes to the absorbance of the layers. In such case, the KramersKronig (KK) consistent Tauc-Lorentz model is expected to be more accurate than the Cauchy-Urbach model. Whereas the latter fits the bandgap absorption tail empirically using the Urbach function (recall Eqn. 2.2), the former, based on its physically valid KKconsistency property, models the dielectric functions above the bandgap energy, while assuming transparency of the material below the bandgap ${ }^{40-41}$. Due to the KK consistency, the real part of the dielectric function $\left(\varepsilon_{1}\right)$ is extracted from the imaginary part $\left(\varepsilon_{2}\right)$. Use of the Tauc-Lorentz model has been reported for estimating the optical bandgap of polycrystalline $\mathrm{GaN}$ films $\mathrm{s}^{38,42}$.

For determining the optical constants of GaN, ex-situ wafer-mapping was performed in the multi-sample analysis (MSA) mode of the CompleteEASE software ${ }^{43}$. In MSA, the same value of optical constants is assumed at all the mapped locations on the wafer, and only the thicknesses are fitted. The MSA-approach minimizes the fit-correlations and increases the fit-accuracy ${ }^{44}$.

The following points summarize the MSA routine used to determine the optical constants of GaN:

Mapping the layer at the wafer-center and at eight locations around it over $1.5 \mathrm{~cm}$ radius.

- Fitting the thicknesses of these points using the Cauchy optical model in the dispersion-free region, with a common Cauchy $A$ parameter for every mapped location.

Fixing the thicknesses, extending the fit over the entire spectral range, and parameterizing the Cauchy model into a KK-consistent Tauc-Lorentz model.

Fitting $\varepsilon 2$ with the Tauc-Lorentz model and determining $\varepsilon 1$ from KK-consistency.

The optical constants $(n, k)$ are determined from $\varepsilon_{1}$ and $\varepsilon_{2}$, respectively. 


\section{Appendix 2.4: Optical emission spectroscopy (OES)}

A 2.4.1 OES of the $\mathrm{NH}_{3}-\mathrm{Ar}$ plasma: OES studies were performed with an Avantes spectrometer $(0.6 \mathrm{~nm}$ resolution) to address the possible cause of the variation in the material properties of the $(\mathrm{Al}) \mathrm{GaN}$ layers with the plasma composition (recall Fig. 2.17 - 2.19). Optical emission of the $\mathrm{NH}_{3}-\mathrm{Ar}$ plasma revealed characteristic lines of atomic hydrogen (i.e., $\left.\mathrm{H}_{\alpha}, \mathrm{H}_{\beta}\right), \mathrm{N}_{2}, \mathrm{~N}_{2}{ }^{+}$and $\mathrm{Ar}$ species. To simplify the attribution of the emission lines, plasmas of pure $\mathrm{H}_{2}, \mathrm{Ar}, \mathrm{N}_{2}$ and $\mathrm{NH}_{3}$ were studied by OES in separate experiments (Fig. A 2.4 a - d). Table A 2.4 lists the characteristic emission lines to be analysed. The emission line of oxygen (as residual gas) is also included.

In pure $\mathrm{NH}_{3}$ plasma (Fig. A 2.4 d), apart from the two at-H lines, the first negative $\left(1 \mathrm{NN}_{2}{ }^{+}\right)$and the second positive $\left(2 \mathrm{PN}_{2}\right)$ systems of $\mathrm{N}_{2}{ }^{+}$and $\mathrm{N}_{2}$, respectively, and the LewisRayleigh glow were observed. Among all $\mathrm{NH}_{\mathrm{x}}(x=1-2)$ radicals, a transition associated with the NH species (i.e., $\mathrm{A}^{3} \Pi-\mathrm{X}^{3} \Sigma^{-},(0-0)$ at $\left.336 \mathrm{~nm}\right)$ is reported ${ }^{45-46}$. This wavelength coincides with the $2 \mathrm{PN}_{2}(0-0)$ transition at $335.8 \mathrm{~nm}$. Nevertheless, the emission of the $\mathrm{NH}$ radicals was difficult to identify due to its low intensity. A comparison of the corresponding spectral region for $\mathrm{N}_{2}$ and $\mathrm{NH}_{3}$ plasmas is shown in the inset of Fig. A 2.4 d.
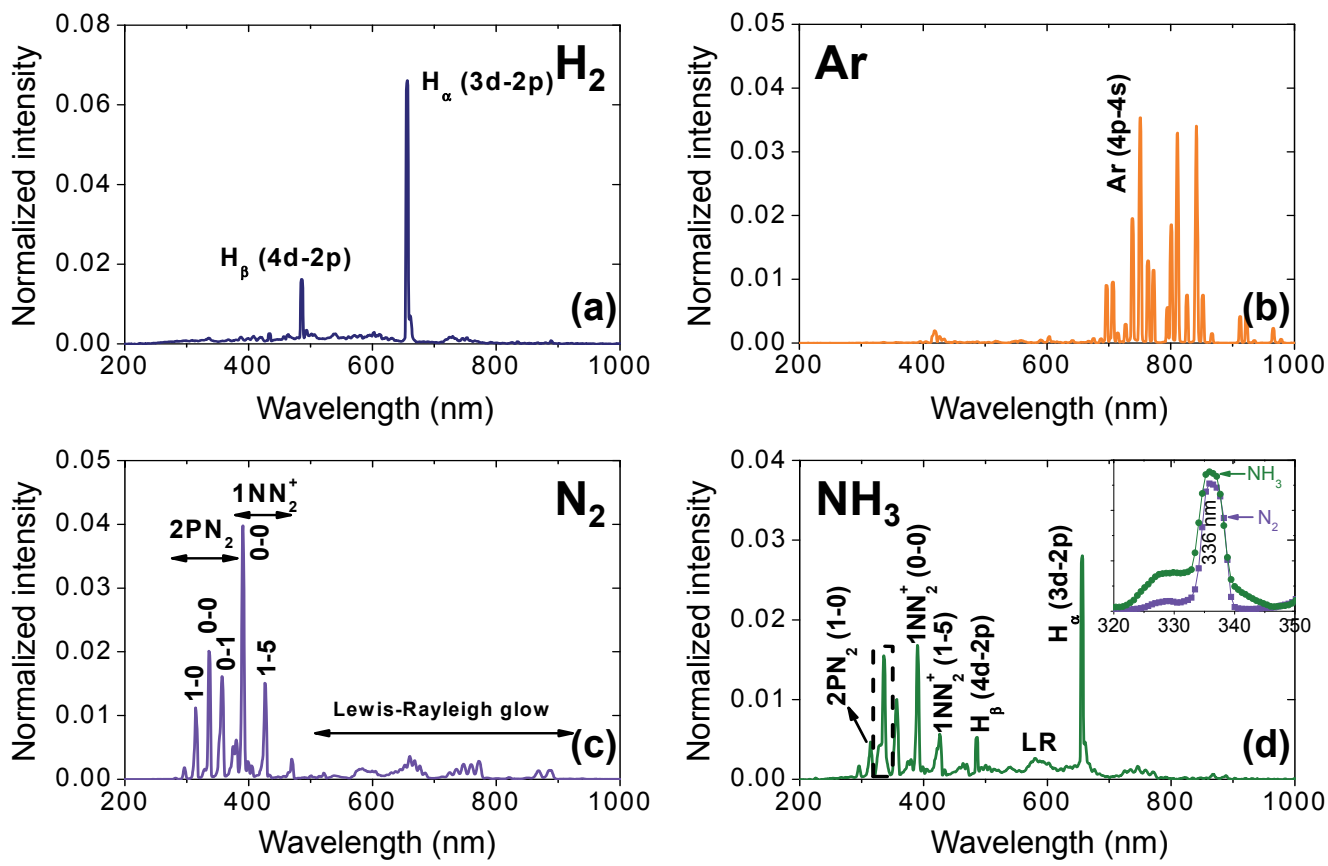

Fig. A 2.4 a-d OES of pure $\mathrm{H}_{2}, \mathrm{Ar}, \mathrm{N}_{2}$ and $\mathrm{NH}_{3}$ plasmas and their characteristic emission lines. 
Fig. A $2.4 \mathbf{e}-\mathbf{h}$ shows the $\mathrm{OES}$ of the $\mathrm{NH}_{3}-\mathrm{Ar}$ plasma and the observed plasma colour at different $\mathrm{NH}_{3}$ partial pressures $\left(P_{\mathrm{NH} 3}\right)$ and mole fractions $\left(x_{\mathrm{NH} 3}\right)$; see Section 2.3.2 for the definition of both. At a high $P_{\mathrm{NH} 3}(15 \mathrm{mbar})$, the colour is strongly violet (e), whereas at low $P_{\mathrm{NH} 3}(2 \mathrm{mbar})$, the colour is strongly orange (h). The change of colour occurs between $P_{\mathrm{NH} 3}$ values of 9 mbar (f) and 5 mbar (g).
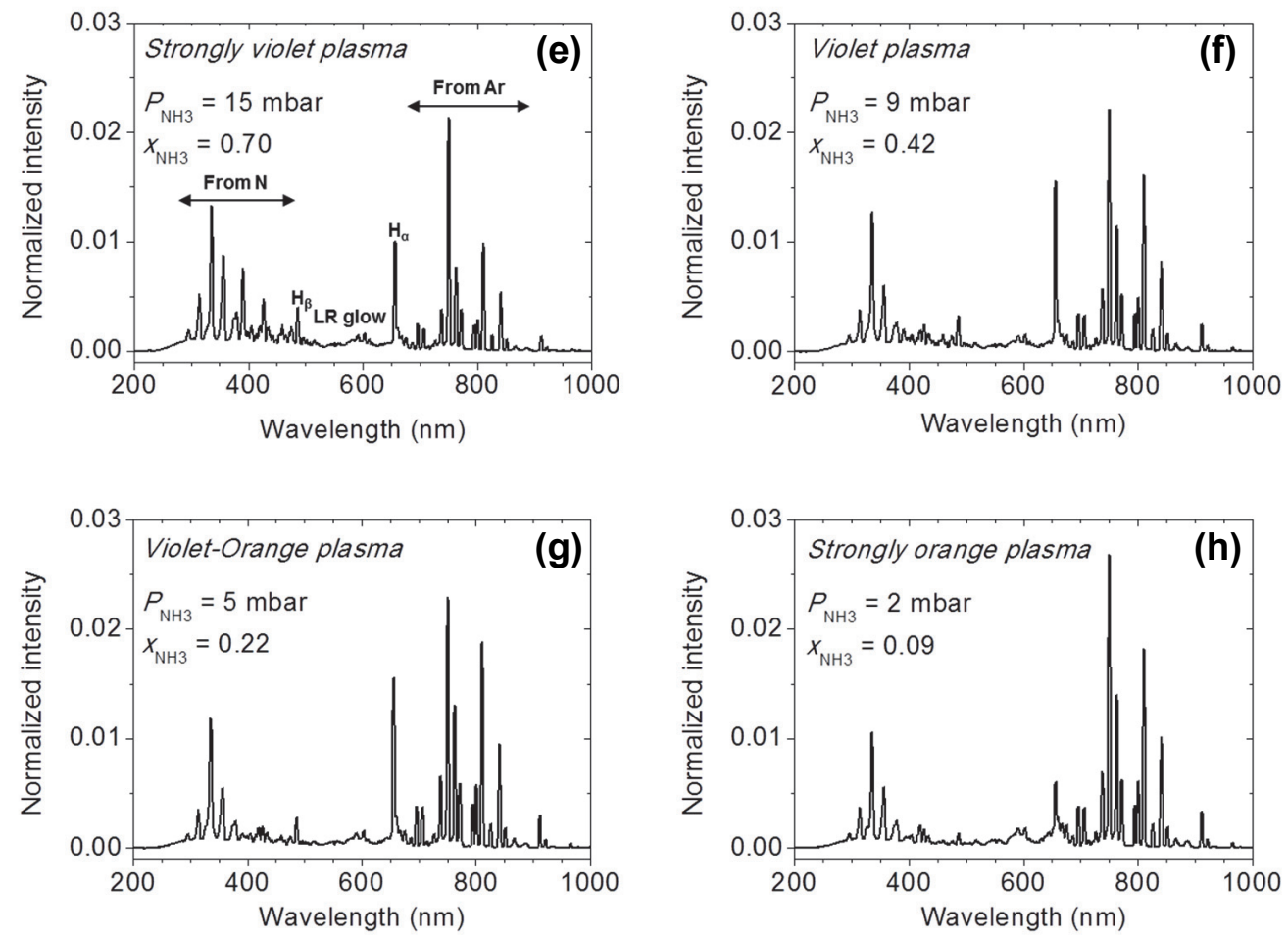

Fig. A 2.4 e - h OES of $\mathrm{NH}_{3}-\mathrm{Ar}$ plasma at different $P_{\mathrm{NH} 3}$ and $x_{\mathrm{NH} 3}$ values. The corresponding plasma colour is also indicated. 
Table A 2.4 List of the relevant emission lines in the OES spectra of Fig. A 2.4 (a - d). $\left(\mathrm{O}_{2}\right.$ was additionally monitored as a source of impurity.)

\begin{tabular}{|c|c|c|c|c|}
\hline Source & Emission line & Transition & $\begin{array}{c}\text { Wavelength } \\
(\mathrm{nm})\end{array}$ & Reference \\
\hline \multirow{2}{*}{$\mathrm{H}_{2}$} & $\mathrm{H}_{\alpha}$ & $3 d-2 p$ & 656.2 & \multirow{2}{*}{ [56] } \\
\hline & $\mathrm{H}_{\beta}$ & $4 d-2 p$ & 485.8 & \\
\hline \multirow{2}{*}{$\operatorname{Ar}$} & \multirow{2}{*}{ - } & $2 p-1 s$ & $695.4-867.5$ & \multirow{2}{*}{ [57] } \\
\hline & & $4 p-4 s$ & 750.5 & \\
\hline \multirow{6}{*}{$\mathrm{N}_{2}$} & $1^{\text {st }}$ negative & $0-0$ & 390.6 & \multirow{5}{*}[45,58]{} \\
\hline & $\begin{array}{l}\text { system } \\
\left(1 \mathrm{NN}_{2}^{+}\right)\end{array}$ & $1-5$ & 426.7 & \\
\hline & \multirow{3}{*}{$\begin{array}{c}2^{\text {nd }} \text { positive } \\
\text { system } \\
\left(2 \mathrm{PN}_{2}\right)\end{array}$} & $1-0$ & 314.3 & \\
\hline & & $0-0$ & 335.8 & \\
\hline & & $0-1$ & 357.3 & \\
\hline & $\begin{array}{c}\text { Lewis- } \\
\text { Rayleigh glow }\end{array}$ & \multicolumn{2}{|c|}{$500-1100 \mathrm{~nm}$} & {$[60,61]$} \\
\hline $\mathrm{O}_{2}$ & - & $4 s-3 p$ & 313.8 & [59] \\
\hline
\end{tabular}

A 2.4.2 Change in the $\mathrm{NH}_{3}-$ Ar plasma composition with $\boldsymbol{P}_{\mathbf{N H} 3}$ : To study the changes in the composition of the $\mathrm{NH}_{3}-\mathrm{Ar}$ plasma with $P_{\mathrm{NH} 3}$, the $\mathrm{H}_{\alpha}(656.2 \mathrm{~nm}), 1 \mathrm{NN}_{2}{ }^{+}(0-0)(390.6 \mathrm{~nm})^{\ddagger}$, and $\operatorname{Ar}(4 \mathrm{p}-4 \mathrm{~s})(750.5 \mathrm{~nm})$ emission lines were monitored (Fig. A $2.4 \mathbf{i}-\mathbf{k})$. To note, the $2 \mathrm{PN}_{2}(0-0)$ line at $335.8 \mathrm{~nm}$ exhibits only a little change with the pressure variation (see Fig. A 2.4 e - h). Besides, $\mathrm{O}_{2}(4 \mathrm{~s}-3 \mathrm{p})$ line at $313.75 \mathrm{~nm}$ was monitored to account for this impurity (1).

Fig. A $2.4 \mathbf{i}-\mathbf{l}$ reveals that increasing the $P_{\mathrm{NH} 3}$ causes: (i) a steep rise (between 0.6 - 5 mbar $\left.P_{\mathrm{NH} 3}\right)$, a brief plateau $\left(5-9\right.$ mbar), and a steady decrease $\left(9-15\right.$ mbar) of the $\mathrm{H}_{\alpha}$ line ${ }^{\S}$; (ii) a gradual (between 0.6 - 9 mbar) and then an abrupt increase $(9-15$ mbar) of the $1 \mathrm{NN}_{2}{ }^{+}$line; (iii) a steady decrease (between $2-15$ mbar) of the Ar line; and (iv) an overall gradual increase (between $0.6-15$ mbar) of the $\mathrm{O}_{2}$ line. The change in the plasma colour from orange to violet with increasing $P_{\mathrm{NH} 3}$ is consistent with the decreasing intensities of the

$\$$ Although the intensity of the Lewis-Rayleigh (LR) afterglow indicates the concentration of atomic nitrogen in the plasma ${ }^{60}$, it is not monitored because a single strong emission line is hardly present in the LR glow. The intensities in Fig. A $2.4 \mathbf{i}$ - l represent peak intensities rather than the integrated areas under them.

$\S$ The plateau between the $P_{\mathrm{NH} 3}$ values of $4.9-8.9$ mbar may suggest that in this pressure range, the $\mathrm{H}$-concentration reached a maximum. 
$\mathrm{H}_{\alpha}$ and $\mathrm{Ar}$ emission lines and the increasing intensity of the $\mathrm{N}_{2}$ emission line, as shown in figures $\mathbf{e}-\mathbf{h}$. The AlN and GaN samples studied in Sections 2.3.2 and 2.3.3 are highlighted as GaN-1, AlN-1, etc., depending on the $P_{\mathrm{NH} 3}$ used for their deposition (see also Table 2.2).
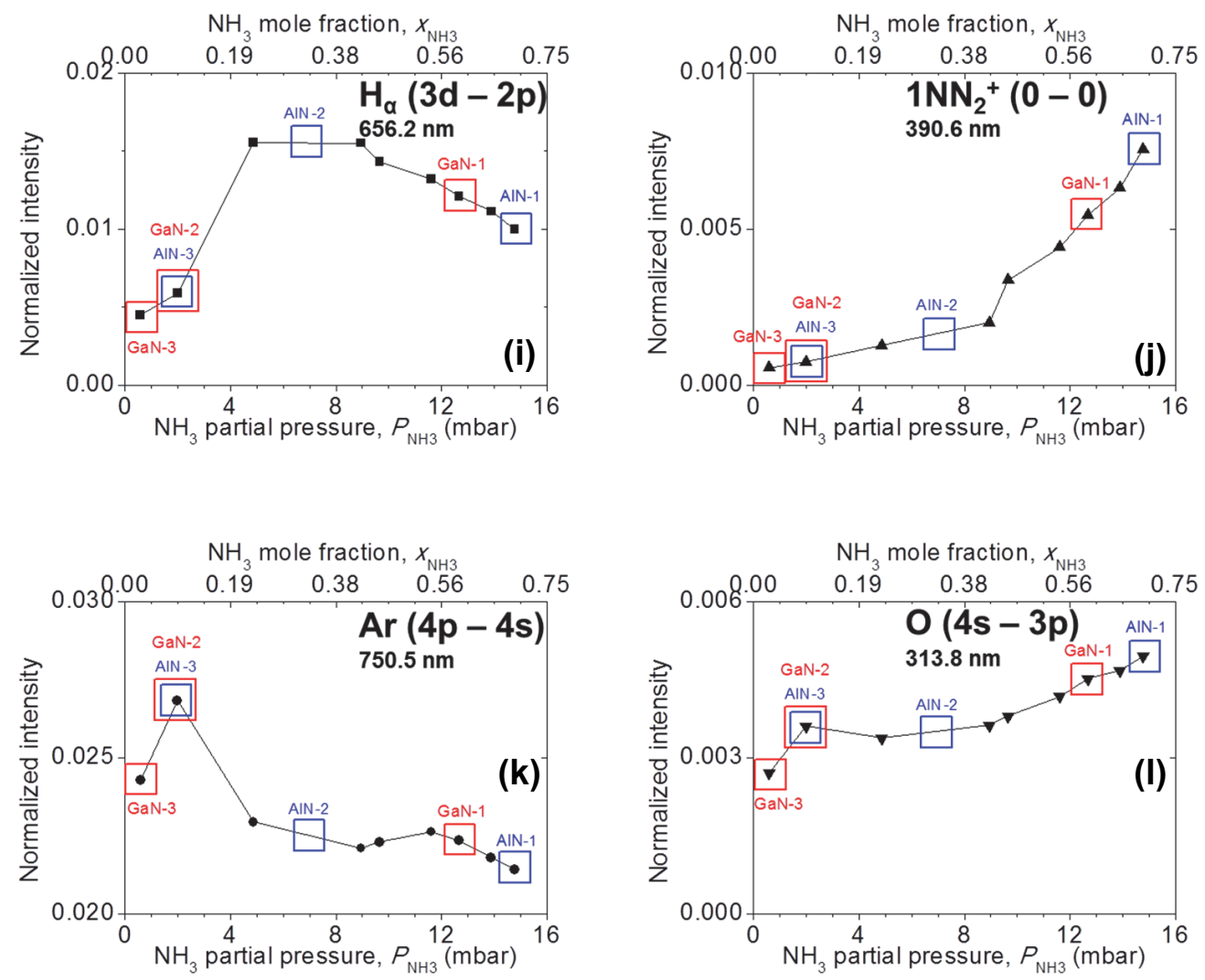

Fig. A 2.4 i - I Variation of intensities of the characteristic emission lines of $\mathrm{H}, \mathrm{N}, \mathrm{Ar}$ and $\mathrm{O}$ with $P_{\mathrm{NH} 3}$. The emission lines and their wavelengths are indicated in the figures. The GaN and AlN samples (described in Sections 2.3.2, 2.3.3) are named GaN-1, AlN-1, etc., and shown by red and blue boxes, respectively.

\section{A 2.4.3 Growth mechanism of PEALD GaN and the influence of plasma composition:} Although the PEALD growth mechanism of (Al)GaN films has not been a focus of this project, to provide an explanation of the observed changes in the polycrystallinity and the optical constants of these layers, a reported mechanism of PEALD GaN growth (Pansila et al. ${ }^{5}$ ) is resorted to (Fig. A $2.4 \mathbf{~ m}$ ). The growth is divided into (i) Adsorption, i.e., the chemisorption of TMG on $-\mathrm{NH}$ and $-\mathrm{GaH}$ surface sites after a TMG pulse, resulting in a $-\mathrm{CH}_{3}$ terminated surface, and (ii) Nitridation, i.e., the de-methylation of the $-\mathrm{CH}_{3}$ 
terminations by $\mathrm{NH}_{2},-\mathrm{NH}$ and $\mathrm{H}$ radicals of the $\mathrm{NH}_{3}$ plasma, with the new surface terminated by these species.

From the above model, we can speculate the relative influence of the radicals on the $\mathrm{GaN}$ growth. For example, the reaction between $-\mathrm{CH}_{3}$ terminated surface (after TMG exposure) and atomic $\mathrm{H}$ is reported to occur through a facile route, requiring little to no activation energy ${ }^{47-48}$. This results in the de-methylation of the surface and a new surface terminated with $\mathrm{Ga}-\mathrm{H}$ or $\mathrm{Ga}-$ dangling bonds. Similar low-activation-energy de-methylation reactions are also reported for TMA and atomic $\mathrm{H}^{49}$. Since these reactions proceed through the Eley-Rideal kinetics (see Chapter 3, Section 3.5), the de-methylation can also be speculated to occur from the physical bombardment of Ar ions of the plasma. Ar atoms excited to their metastable states can additionally cause large energy flux to the growth surface due to inelastic collisions.

During the PEALD of GaN at low $P_{\mathrm{NH} 3}$ (samples GaN-3, GaN-2), the OES indicates a low emission of both $\mathrm{N}_{2}$ and $\mathrm{N}_{2}{ }^{+}$. Comparatively, the $\mathrm{H}_{\alpha}$ line is of a higher intensity, and so is the Ar line. The higher intensities may be correlated to a higher concentration of at-H and metastable Ar atoms (or Ar ions) in the plasma. In the PEALD of AlN, sample AlN-3 is deposited under the same $P_{\mathrm{NH} 3}$ as GaN-2, whereas AlN-2 is deposited under a presumably higher at-H concentration (perhaps, coincidentally reaching the maximum). For these samples, due to the low intensities of the $\mathrm{N}_{2}$ and $\mathrm{N}_{2}{ }^{+}$lines (both are the measure of $\mathrm{NH}_{3}$ dissociation or its "activation" in the plasma), an inefficient nitridation may occur.

Subsequently, some of the $-\mathrm{CH}_{3}$ terminations can be de-methylated by the at- $\mathrm{H}$ and/or the Ar flux, resulting in $\mathrm{Ga}-$ and $\mathrm{Al}-$ surfaces with or without H-terminations. Such sites would still allow the chemisorption of TMG (Fig. A 2.4 m). After several ALD cycles, the coverage of such de-methylated sites is expected to increase, resulting in $\mathrm{Ga}$ and $\mathrm{Al}$ clusters, respectively. These clusters could intervene in the GaN crystal formation, thereby degrading the layer crystallinity almost to the level of amorphousity, as observed. Besides, the clusters can also be oxidized in the presence of the background oxygen. On the contrary, samples GaN-1 and AlN-1 deposited under the conditions giving high intensities of the $\mathrm{N}_{2}$ and $\mathrm{N}_{2}{ }^{+}$lines and low intensities of the $\mathrm{H}_{\alpha}$ and $\mathrm{Ar}$ lines, would experience an efficient nitridation. Combined with a correspondingly reduced amount of $\mathrm{Ga}$ and $\mathrm{Al}$ clusters, this is expected to result in (A1)GaN layers of enhanced crystallinity.

Finally, the increased optical bandgaps and low refractive indices of samples GaN-2, GaN-3 and AlN-2 and AlN-3 are a consequence of their amorphous nature. For example, increase in the optical bandgap of poly-GaN layers with decreasing crystallinity has been variously reported ${ }^{38,50-51}$. The reason has been attributed to the increase of disorder in the material; with the bandgaps increasing from 3.45 to $3.90^{50}$, and from 3.90 to $3.95 \mathrm{eV}^{38}$. 
Similar trends have been reported for poly- $\mathrm{AlN}^{52}$ and poly-Si ${ }^{53}$ films. The probable oxidation of the clusters may additionally contribute to the larger bandgaps and lower refractive indices.

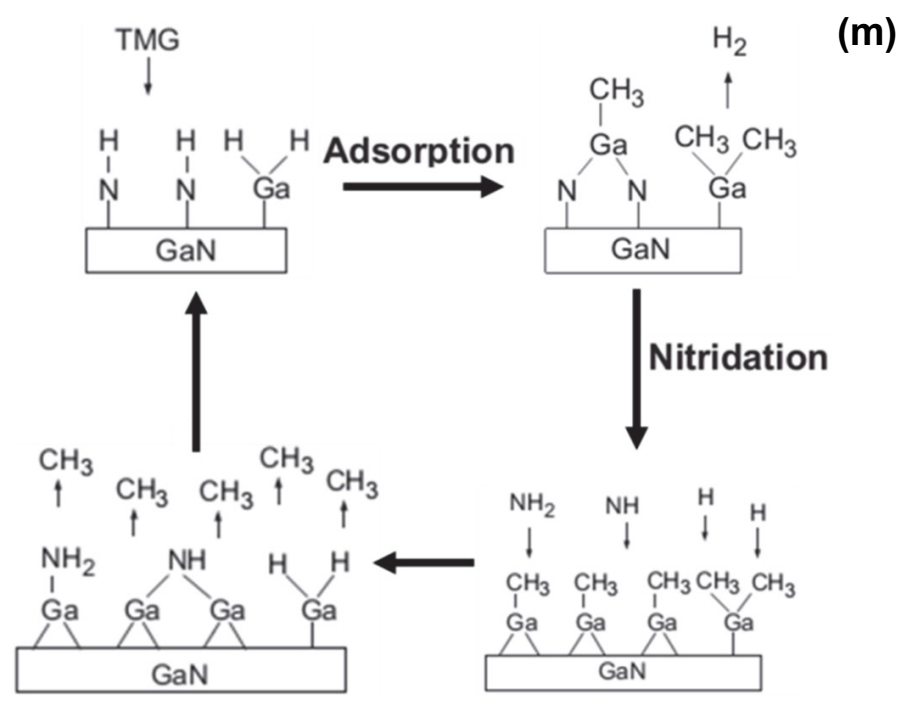

Fig. A 2.4 m Schematic of PEALD GaN growth from TMG and $\mathrm{NH}_{3}$-plasma. (Original source: reference $)^{5}$. 


\section{Towards ALD of AlN and GaN}

using radicals generated by a
hot-wire*

${ }^{*}$ This chapter is an extended version of:

i. A. Kovalgin, M. Yang, S. Banerjee, R. O. Apaydin, Antonius A. I. Aarnink, S. Kinge and R. A. M. Wolters, Advanced Materials Interfaces, 2017, 4(18), 1700058.

ii. S. Banerjee, R. O. Apaydin, A. A. I. Aarnink, J. Schmitz, D. J. Gravesteijn and Alexey Y. Kovalgin, EuroCVD 21-Baltic ALD 15 Conf., Linköping, June 2017. 


\subsection{Introduction}

Hot-wire assisted atomic layer deposition (HWALD) is a relatively novel technique $^{1}$ that has the potential to overcome some of the inherent limitations of plasmaenhanced (PE) ALD, such as the generation of charged particles and high-energy neutrals, while still supplying the radicals which participate in the ALD reactions. This alternative-toplasma approach employs a metallic filament (e.g., tungsten) that is resistively heated, usually up to temperatures of $1300-2000{ }^{\circ} \mathrm{C}$. Impinging precursor molecules (such as $\mathrm{H}_{2}$ and $\mathrm{NH}_{3}$ ) dissociate catalytically on the filament, generating radicals. Charged particles such as ions and electrons are not generated. Recently, this method has been successfully utilized for the ALD of metals, such as $\mathrm{Ni}$ and $\mathrm{W}^{2-4}$. However, except for an old report ${ }^{5}$, HWALD of $\mathrm{GaN}$ (or AlN) has not been utilized so far. This makes room for a renewed research interest in this area.

The rest of this chapter is classified into two broad sections: Section 3.2 and Section 3.3 - 3.4. The former section deals with the various tests to characterize radical generation from $\mathrm{H}_{2}$ and $\mathrm{NH}_{3}$ sources. This knowledge has been applied towards the ALD of AlN and GaN films, as addressed in the latter sections. More specifically, Section 3.2.1 describes the production and delivery of atomic hydrogen (at-H) radicals, produced from the dissociation of molecular hydrogen $\left(\mathrm{H}_{2}\right)$. Section 3.2.2 describes the same for the various radicals generated from ammonia. Both the sub-sections address the influence of placing the HW in the direct line-of-sight (L-O-S) and outside the line-of-sight with the substrate, on the delivery of the radicals to the substrate. Section $\mathbf{3 . 3}$ discusses targeted HWALD of AlN, in- and outside the L-O-S conditions. Its sub-sections address (i) role of oxygen contamination in HWALD, and (ii) distinguishing between HW-assisted and thermal ALD AlN films. Section 3.4 and its sub-sections detail the results of targeted HW-GaN ALD in and outside the L-O-S conditions, and reveals that due to the high reactivity of at- $\mathrm{H}$ (compared to $\mathrm{NH}_{\mathrm{x}}$ ), the formation of elemental $\mathrm{Ga}$ is favoured over that of GaN. Section 3.5 proposes a reason behind the higher reactivity of at-H. Section 3.6 concludes the chapter.

\subsubsection{The hot-wire hardware}

The HW is a $23 \mathrm{~mm}$ tungsten coil (Fig. 3.1 a), encased in a ceramic tubing (not shown). Fig. 3.1 b shows a picture of the glowing filament inside the reactor. The HW is located outside the L-O-S in the picture. The schematics of the positioning of the HW are shown in Fig. 3.2 a and b, respectively. The filament temperature, as a function of power, was reported in a previous work ${ }^{6}$. Even in L-O-S position, heating the $\mathrm{HW}$ to $1800^{\circ} \mathrm{C}$ hardly affected the wafer temperature (Fig. $3.2 \mathrm{c}$ ). 

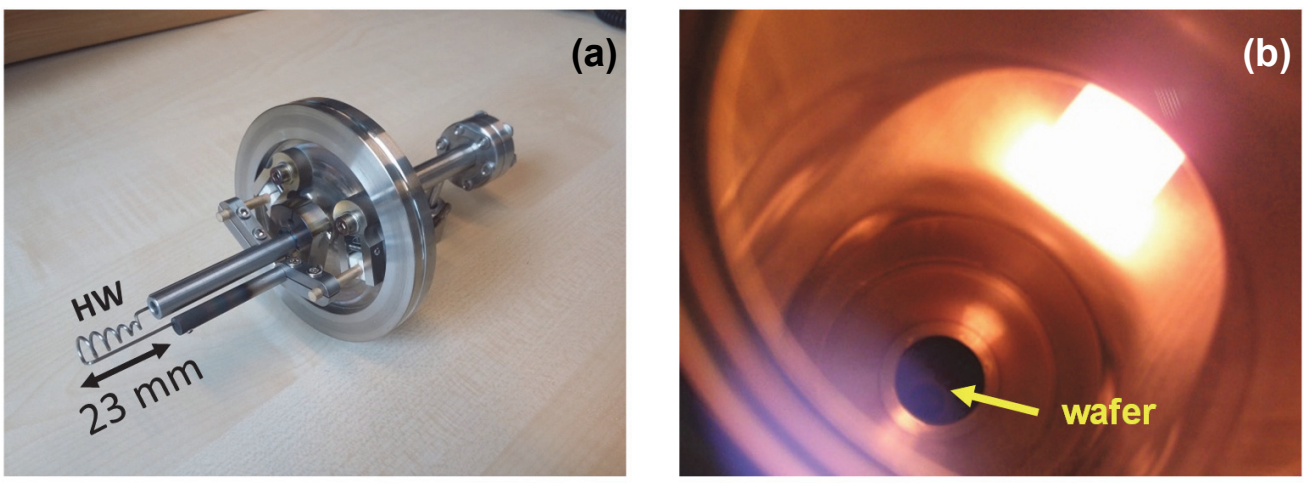

Fig. 3.1 (a) Hardware of the HW (b) Glowing HW in the reactor, positioned outside the L-O-S.
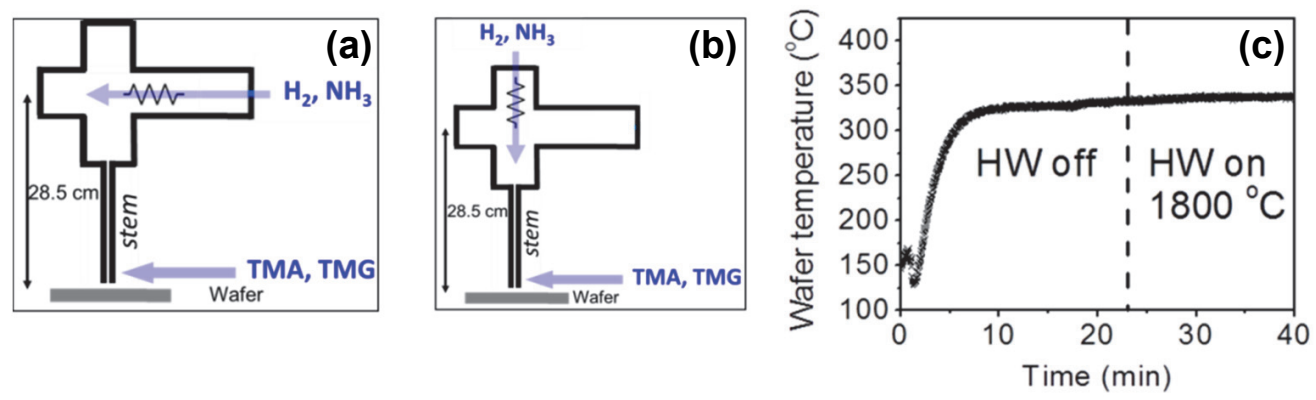

Fig. 3.2 Schematic of the wafer and HW position in (a) outside the L-O-S, (b) in the L-O-S. The 'stem' refers to the tube connecting the HW to the wafer. (c) Evolution of wafer temperature before and after switching on the HW, as monitored with in-situ SE. The initial rise in the temperature (0-10 min) reflects the wafer heating up to the desired deposition temperature. No measureable temperature increase was observed after switching on the HW.

\subsection{Tests for radical generation}

\subsubsection{Catalytic dissociation of $\mathrm{H}_{2}$ : Generation of at-H radicals}

It is well-established that molecular $\mathrm{H}_{2}$ can dissociate upon adsorption on a W filament surface at high temperatures into atomic-H radicals ${ }^{7}$. However, whether the asgenerated at-H radicals can be delivered to the substrate in sufficient amounts, without recombination, needs to be investigated in order to perform future HW-assisted ALD experiments. Recombination of at-H radicals (into molecular $\mathrm{H}_{2}$ ) occurs from the collision between two radicals and a third body; the latter may be another at-H radical or the reactor wall ${ }^{8}$. 
3.2.1.1 In-situ monitoring Te etching by at-H radicals: The actual presence of at-H at the substrate can be proven by its etching action on a Te film. At-H reacts with Te through the reaction: $\mathrm{Te}+2 \mathrm{H} \rightleftharpoons \mathrm{H}_{2} \mathrm{Te} \uparrow$. The produced hydrogen telluride $\left(\mathrm{H}_{2} \mathrm{Te}\right)$ is volatile in nature ${ }^{9}$. The reaction can occur at room temperature, and its rate decreases with temperature due to the regeneration of Te. Importantly, this reaction only occurs with at- $\mathrm{H}$, and not with molecular $\mathrm{H}_{2}{ }^{9}$, therefore making Te a sensor of at-H.

In-situ monitoring of the etching of a sputtered-Te film on a $\mathrm{Si}$ wafer by spectroscopic ellipsometry (SE), on introducing $\mathrm{H}_{2}$ via the $\mathrm{HW}$, indicated the generation as well as the delivery of at-H radicals to the wafer. Initial thicknesses of the Te films were typically a few tens-of-nanometers. The etching of Te, as recorded by in-situ SE, is shown in Fig. 3.3 a, red; the HW being in the L-O-S position. The delivery of at-H was also observed outside the L-O-S position in a separate experiment. The etch-rate in the L-O-S position was $4.4 \mathrm{~nm} / \mathrm{min}$ at $4 \times 10^{-3}$ mbar reactor pressure.

\subsubsection{Thermal catalytic dissociation of $\mathrm{NH}_{3}$}

3.2.2.1 Generation of at-H radicals: It is known that $\mathrm{NH}_{3}$ can also dissociate on a hot $\mathrm{W}$ filament ${ }^{10-11}$, producing at- $\mathrm{H}$ and $\mathrm{N}$-containing radicals. The latter is generalized as $\mathrm{NH}_{\mathrm{x}}$ radicals with $x=0-2$; however, mainly $\mathrm{NH}_{2}$ radicals and small amounts of molecular $\mathrm{N}_{2}$ and $\mathrm{H}_{2}$ are present ${ }^{10}$. Their relative amounts depend on the filament temperature and pressure.

The generation of at- $\mathrm{H}$ from the dissociation of $\mathrm{NH}_{3}$ was confirmed from the etching of Te films (Fig. 3.3 a, blue). The etch-rate was observed to be $\sim 5 \mathrm{x}$ lower than from $\mathrm{H}_{2}$, at $4 \times 10^{-3}$ mbar. The reason may be due to a lower dissociation efficiency of $\mathrm{NH}_{3}$ compared to $\mathrm{H}_{2}$, and/or an enhanced recombination of the as-generated at-H radicals with the various other species (e.g., $\mathrm{NH}_{3}, \mathrm{H}_{2}$ and $\mathrm{NH}_{\mathrm{x}}$ radicals) ${ }^{12}$. Fig. 3.3 b compares the Te etch rates, by at-H generated from $\mathrm{H}_{2}$ and $\mathrm{NH}_{3}$ sources, at several reactor pressures. The experiment was performed in the L-O-S position. Higher pressures presumably lead to a higher recombination rate, therefore reducing the etch-rate. Whereas below $5 \times 10^{-2}$ mbar, the etch-rate using $\mathrm{H}_{2}$ is significantly higher than that using $\mathrm{NH}_{3}$; beyond this pressure, almost equal rates are observed. Further, beyond $5 \times 10^{-1} \mathrm{mbar}$, hardly any etching is detected from both the sources, indicating that at-H recombines completely.

The controlled generation of at- $\mathrm{H}$ during the introduction of $\mathrm{NH}_{3}$ pulses, and its efficient removal by purging, was revealed from the stepwise etching of a Te film, as monitored by in-situ SE (Fig. 3.3 c). In this experiment, $\mathrm{NH}_{3}$ was introduced in $2.5 \mathrm{~s}$ long pulses with an Ar carrier background. The purge duration was $10 \mathrm{~s}$. The possibility of the 
production of at-H in such a controllable (i.e., pulsed) manner can enable HWALD using this radical.
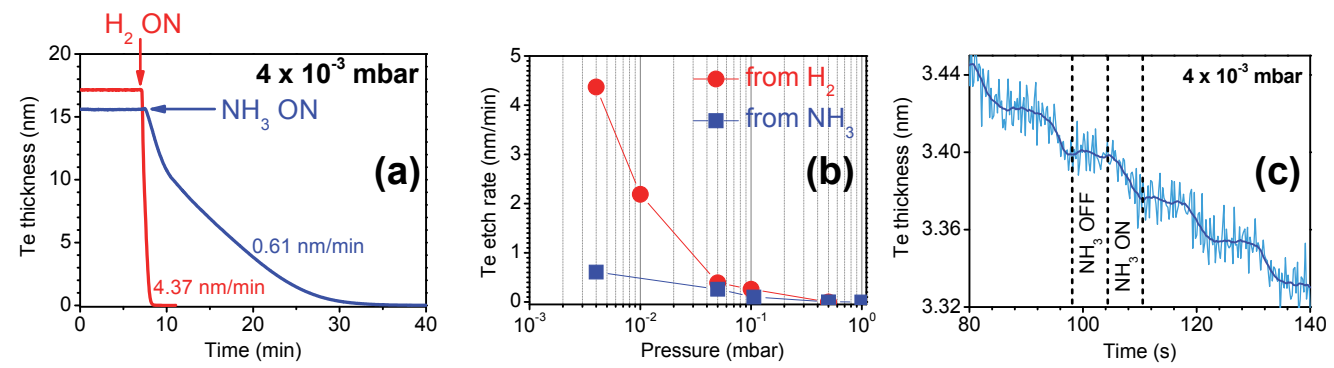

Fig. 3.3 Comparison of Te etching from $\mathrm{H}_{2}$ and $\mathrm{NH}_{3}$ as a function of (a) Time (b) Reactor pressure. (c) Step-wise etching of Te confirms controlled at-H generation (from $\mathrm{NH}_{3}$ pulses) and removal. Experiments $\mathbf{a}-\mathbf{c}$ were performed in the L-O-S position, and the filament temperature was $1800^{\circ} \mathrm{C}$.

3.2.2.2 Generation of $\mathrm{NH}_{\boldsymbol{x}}(\boldsymbol{x}=\mathbf{0}-2)$ radicals: The generation of $\mathrm{NH}_{\mathrm{x}}$ (i.e., principally $\mathrm{NH}_{2}$ ) radicals from the dissociation of $\mathrm{NH}_{3}$ and their delivery to the substrate, were investigated by the nitridation of a Si wafer, which was expected to result in the formation of a $\mathrm{SiN}_{\mathrm{x}}$ layer ${ }^{13}$. For the nitridation experiments, the wafer temperature was $250{ }^{\circ} \mathrm{C}$.

The effects of the HW positioned outside and in the L-O-S had profound influence on the delivery of the $\mathrm{NH}_{\mathrm{x}}$ radicals to the wafer (Fig. 3.4 a). Nitridation occurred only in the L-O-S position, as suggested by the thickness increase (in light and dark blue) at different pressures. Outside the L-O-S, a noticeably lower thickness increase (in red) suggested that the delivery of the radicals was in a much lesser amount. To test that the thickness increase was caused by the $\mathrm{NH}_{\mathrm{x}}$ radicals only (and not by molecular $\mathrm{NH}_{3}$ ), the nitridation was also attempted thermally, without HW action. Only a negligible thickness increase was observed (in black). To note: at- $\mathrm{H}$, produced from the $\mathrm{NH}_{3}$, was not expected to cause any film formation on $\mathrm{Si}$, and hence, cause thickness increase, since it is reported to etch $\mathrm{Si}^{14}$.

The effect of the HW positioned outside the L-O-S can be compared with the thermal experiment. The similar thickness increase indicates that almost the entirety of the $\mathrm{NH}_{\mathrm{x}}$ radicals, generated outside the L-O-S, was depleted during making the $90^{\circ}$ turn into the stem (refer to Fig. 3.2 a). The presence of the large dead volumes at the turn could have also contributed to their recombination, acting as gettering sites. On the contrary, in L-O-S, the substrate was exposed to the direct $\mathrm{NH}_{\mathrm{x}}$ beam, minimizing the recombination.

A pressure-dependence of the nitridation in the L-O-S position was also observed (dark and light blue curves). A higher pressure enhanced the recombination of the radicals in their path between the HW and the wafer, leading to a lower thickness increase, and vice 
versa. (To note: the thicknesses are obtained from in-situ SE monitoring, and are not absolute values, but only give a qualitative indication.)

To verify that the film formation in the L-O-S position was due to nitridation (and not due to oxidation from $\mathrm{O}_{2}$ and $\mathrm{H}_{2} \mathrm{O}$ present in $\mathrm{Ar}$ and $\mathrm{NH}_{3}$ ), carrier-Ar was flowed via the HW in the L-O-S setup. However, no thickness increase was observed before or after turning the HW ON (Fig. 3.4 b, orange). When $\mathrm{NH}_{3}$ was introduced instead (in blue), an immediate thickness increase was noted on switching on the HW. Based on the assumption that $\mathrm{NH}_{3}$ and Ar contain similar levels of oxidants, the thickness increase from the HW action (in Fig. 3.4 a and b) is assigned to the formation of a $\mathrm{SiN}_{\mathrm{x}}$ film rather than a $\mathrm{SiO}_{\mathrm{x}}$ film. This was further confirmed by XPS (Fig. 3.5 a, b), showing the presence of $\mathrm{Si}-\mathrm{N}$ bonds.
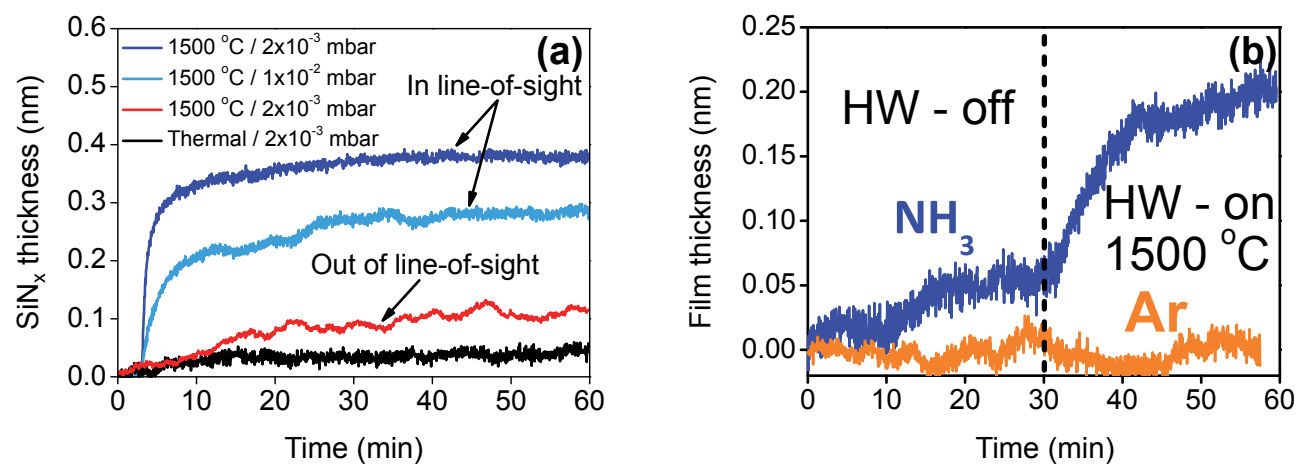

Fig. 3.4 Si-nitridation tests from (a) HW-dissociated- $\mathrm{NH}_{3}$ at outside and in the L-O-S positions. The result of thermal nitridation is also shown for comparison. (b) $\mathrm{Ar}$ (in orange) and $\mathrm{NH}_{3}$ (in blue) supplied via the HW in the L-O-S position. The thicknesses are not absolute, but rather qualitative.
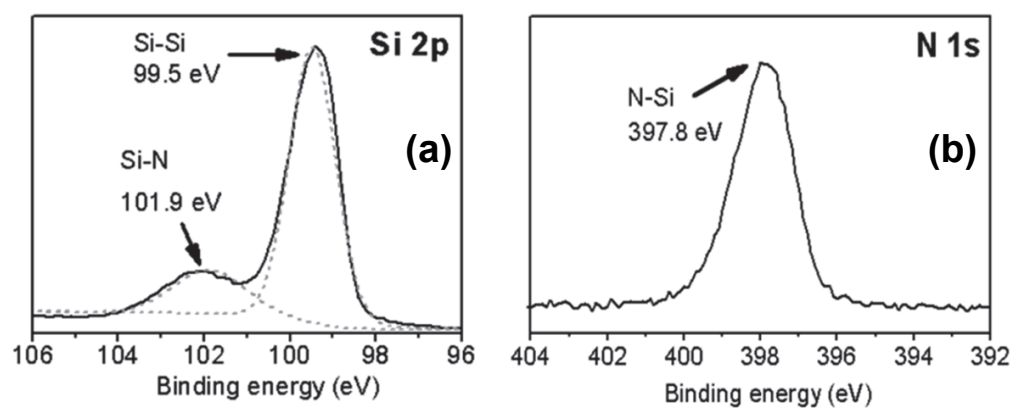

Fig. 3.5 Formation of $\mathrm{SiN}_{\mathrm{x}}$ film from nitridation in the L-O-S position, revealed from XPS survey scan. 


\subsection{HWALD of AIN and oxygen contamination}

When using a radical-assisted approach such as HWALD, considerations must be taken to avoid / suppress the unwanted (parasitic) reactions that involve the radicals.

In the HWALD of AlN from TMA and $\mathrm{NH}_{3}$ precursors, the chance of the chemisorbed-TMA for getting de-methylated by the at-H flux into Al-with-dangling-bonds species can be significant (a possible cause behind this is addressed in Section 3.5). Such a pure-metallic surface can be highly reactive. The presence of the background oxidants in the reactor can only be minimized in ultrahigh vacuum systems, such as with a base pressure of $10^{-9}$ mbar or below. Standard turbomolecular pump-based deposition systems (such as the used ALD reactor) have a base pressure of the order $10^{-7}$ mbar, enabling a flux of $\sim 0.1 \mathrm{monolayer} / \mathrm{s}$ of oxidants to the substrate. A $10 \mathrm{~s}$ purge time in the ALD recipe therefore doses the surface with $\sim 1$ monolayer of oxidants. The action of HW on the oxidants, producing O-containing radicals, additionally contributes to the oxidation of the surface. This makes HWALD of AlN highly challenging, especially when the growth mechanism implies the formation of a free-Al surface as one of the intermediate steps.

\subsubsection{Deposition outside the L-O-S position}

With the HW situated outside the L-O-S (recall Fig. 3.2 a), an experiment was performed where the initially thermal AIN film $(25 \mathrm{~nm})$ was grown, and then the HW was turned on at $1800{ }^{\circ} \mathrm{C}$ filament temperature. The ALD recipe in both cases was: $0.5 \mathrm{~s}$ TMA / $10 \mathrm{~s} \mathrm{Ar} / 2.5 \mathrm{~s} \mathrm{NH}_{3} / 10 \mathrm{~s} \mathrm{Ar}$, at a wafer temperature of $350{ }^{\circ} \mathrm{C}$ and at $10^{-2}$ mbar reactor pressure.

The depth profiled composition of this film (Fig. 3.6 a) supports the previous considerations. Whereas in the duration of the thermal ALD (i.e., before turning the HW $\mathrm{ON}$ ), AlN was formed with only $\sim 5$ atomic $\%$ oxygen, switching on the HW resulted in the formation of pure $\mathrm{Al}$ oxide. The dramatic increase of the oxygen content on switching the $\mathrm{HW}$ on indicates the reduction of the growing surface by at-H (i.e., de-methylation) and its subsequent oxidation.

An ex-situ SE map reveals a radial distribution of the refractive index of the (HWdeposited part of the) layer (Fig. 3.6 b) (to note: the AlN formed during the thermal part was included in the SE model). The refractive index $(n)$ variation can be correlated with the relative $\mathrm{O}$ - and $\mathrm{N}$ - contents, since pure $\mathrm{ALD} \mathrm{Al}_{2} \mathrm{O}_{3}$ has an index between $1.6-1.65$, and pure ALD AlN has an index between 1.95 and 2.0. This is confirmed by measuring $n$ of these films prepared by ALD in separate experiments (Appendix 3.1 shows the correlation between the composition and the index). Fig. 3.6 b shows that at the wafer-center (i.e., just 
below the HW stem), completely $\mathrm{N}$-free $\mathrm{Al}$-oxide was deposited. Moving radially outside the center, an Al-oxynitride $\left(\mathrm{AlO}_{\mathrm{x}} \mathrm{N}_{\mathrm{y}}\right)$ layer, with a gradually decreasing $\mathrm{O}$ - (and increasing $\mathrm{N}$-) content, was formed. At the edge of the wafer, there was a thin ring of pure AlN. The XPS of Fig. 3.6 a was made at the center.

As the origin of the oxide / oxynitride formation is the de-methylation of chemisorbed TMA by at-H radicals (and its subsequent oxidation), the variation of $n$ can be attributed to the gradual depletion of at- $\mathrm{H}$ and/or the oxidants (in molecular and/or radical form) from the center to the edge. Furthermore, since $\mathrm{NH}_{\mathrm{x}}$ radicals were not delivered in this outside-the-L-O-S experiment (recall Fig. 3.4 a), the formation of the pure oxide ring at the center shows that, when radicals were present in maximum amounts, hardly any nitridation could occur from the molecular $\mathrm{NH}_{3}$. The gradual depletion of the radicals from center to the edge correlates with an increasing $\mathrm{N}$-content from the action of $\mathrm{NH}_{3}$.
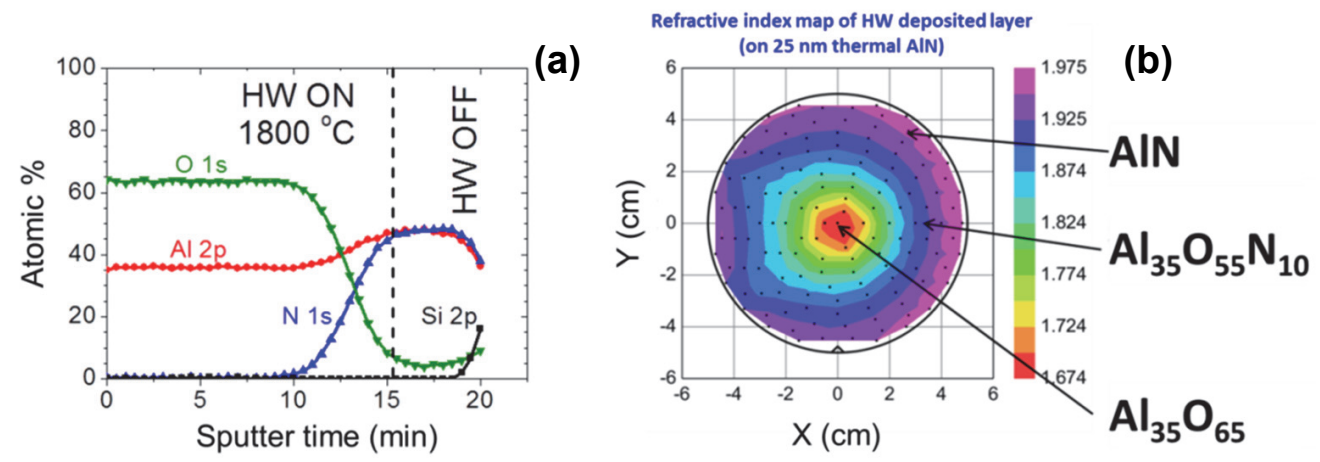

Fig. 3.6 (a) Depth profiled XPS composition of the film formed before and after tuning ON the HW. (b) Refractive index map (by ex-situ SE) of the HW-deposited part of the layer.The compositions are from XPS.

\subsubsection{Deposition in the L-O-S position}

In the L-O-S setup (recall Fig. 3.2 b), the deposition result was different. In this experiment, the HW was ON from the beginning of the ALD, unlike in the previous. At the wafer-center, besides the dominating presence of oxygen, the $\mathrm{N}$-content has increased to 7 at. $\%$ (Fig. 3.7 a). This can be explained by the delivery of $\mathrm{NH}_{\mathrm{x}}$ radicals in the L-O-S. The wafer revealed a sharply-defined grey ring at the center, and an almost uniform blue color elsewhere (Fig. 3.7 b). Depth-profiled XPS peformed at different radial distances from the center (Fig. 3.7 c) revealed a sharp decay of the O-content (and increase of the N-content) from the center outwards. Whereas the grey ring is Al-oxynitride, pure AlN (with $<2$ at. \% oxygen) is deposited immediately outside the ring. Comparing with Fig. 3.6 b, in the L-O-S (i.e., when $\mathrm{NH}_{\mathrm{x}}$ radicals are delivered), nitridation can partially occur at the center, and 
further, the radial depletion of the at-H / O-containing radicals occurs in a much more abrupt fashion. In order to gain an insight into the such-observed competition between the at- $\mathrm{H}$ (and/or O-containing radicals) and the $\mathrm{NH}_{\mathrm{x}}$ radicals, the following sets of experiments were performed.
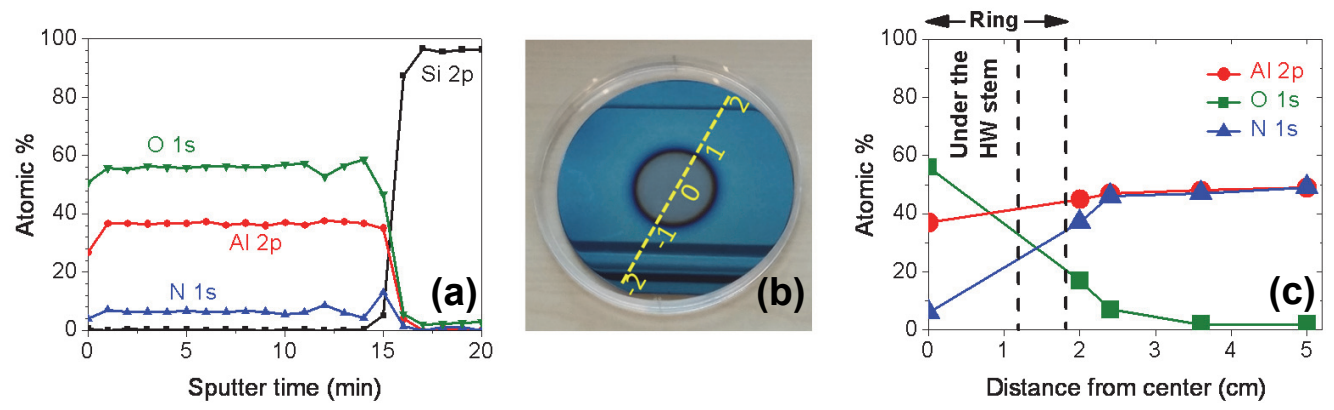

Fig. 3.7 (a) Depth profile composition of the layer deposited in the L-O-S positon, as probed at the wafer-center, (b) Picture of the wafer showing the formation of a well-defined oxynitride ring at the center (in grey) and AlN elsewhere (in blue). The yellow line shows the distance from the center, in centimetres. (c) XPS film compositon along the yellow dotted line of $\mathbf{b}$.

3.3.2.1 Effect of filament temperature: The diameter of the oxynitride ring increased with raising the filament temperature. Additionally, the oxynitride composition also showed consistent changes, by a decrease in the N-content. These were obtained by mapping the wafer with ex-situ SE along its diameter (as shown by the yellow line in Fig. 3.7 b), and then determining the refractive index (and hence the film composition) along the axis. The result is shown in Fig. 3.8 a. The drop in the index at the center implies the formation of an oxide or an oxynitride ring.

Whereas a filament temperature of $1800{ }^{\circ} \mathrm{C}$ produced a wide oxide ring that stretched almost to the wafer edge (in orange), at $1500{ }^{\circ} \mathrm{C}$, the ring shortened greatly and its $\mathrm{N}$-content also increased (in green). Immediately outside the ring, O-free AlN was formed. At $1300{ }^{\circ} \mathrm{C}$, a ring was not observed any more (in violet), and AlN was formed everywhere on the wafer. It will be shown that the material property of this HW-deposited AlN is different from its thermally-deposited counterpart, serving to distinguish between them.

The $1300{ }^{\circ} \mathrm{C}$ experiment partially answers the question whether molecular oxidants (i.e., $\mathrm{H}_{2} \mathrm{O}$ and $\mathrm{O}_{2}$ ) or O-containing radicals (i.e., $\mathrm{O}, \mathrm{OH}$ ) cause the formation of the oxide/oxynitride ring. Previously, at $1300{ }^{\circ} \mathrm{C}$, Te films were observed to etch from $\mathrm{NH}_{3}$ dissociation, implying that at- $\mathrm{H}$ could be produced from $\mathrm{NH}_{3}$ at this filament temperature. During the AIN ALD, the presence of the at-H would cause the de-methylation of TMA, and its oxidation. However, Fig. 3.8 a shows that hardly any oxide formation occurs at this 
temperature. Therefore, molecular oxidants do not cause oxidation of the demethylated TMA; rather O-containing radicals can be the cause. This also implies that $1300{ }^{\circ} \mathrm{C}$ is below the threshold for the generation of these radicals from the molecular oxidants. On the contrary, the increasing O-content of the ring at higher filament temperatures is consistent with the enhanced production of these radicals.

In Fig. 3.7 b and Fig. 3.8 a, the abrupt transition from the oxide/oxynitride to pure AlN beyond the ring can be either from the rapid depletion of at-H or the O-containing radicals. However, under similar experimental conditions, Te-etching by at-H was seen to occur over the entire wafer surface. Therefore, a rapidly decreasing amount of the O-containing radicals is likely the cause of the sharp, well-defined ring.

3.3.2.2 Change in crystallinity with position on the wafer: The radicals $\left(a t-H, \mathrm{NH}_{\mathrm{x}}\right)$ affected the (poly)crystallinity of HW-AlN, which differed from thermally deposited AlN. This was revealed from GIXRD scans.

Fig. 3.8 b compares GIXRD spectra of the two types. HW-AlN was deposited at $1300{ }^{\circ} \mathrm{C}$ and $1500{ }^{\circ} \mathrm{C}$ filament temperature. For the $1500^{\circ} \mathrm{C}$ sample, GIXRD was performed at $3.5 \mathrm{~cm}$ away from the wafer-center, outside the oxynitride ring (in green). At the same position, GIXRD was performed on the $1300{ }^{\circ} \mathrm{C}$ sample (in blue). GIXRD was also performed at the center of this sample (in red) for comparison. The GIXRD of the thermal AlN sample is shown in black.

Although all samples (thermal and HW) revealed hexagonal (wurtzitic) crystallinity, the formation of the (002) crystal planes, relative to the (100) and (101) planes, was suppressed by the radicals. In Fig. $3.8 \mathrm{~b}$, this has been quantified by the ratio of intensities of the (002) and (101) planes $\left(I_{002} / I_{101}\right)$. Whereas the center of the $1300{ }^{\circ} \mathrm{C}$ sample has $I_{002} / I_{101}$ of 0.39 , at $3.5 \mathrm{~cm}$ from the center, the ratio increases to 0.85 , and approaches to that of thermally deposited AlN (0.97). At the same $3.5 \mathrm{~cm}$ position, the $1500{ }^{\circ} \mathrm{C}$ sample has a lower $I_{002} / I_{101}$ of 0.41 . This shows that the radicals tend to form AlN layers with a lower relative fraction of the (002) planes. Increasing the filament temperature enhances the production of radicals, whereas increasing the distance from the center depletes the radicals due to recombination, consequently changing the polycrystallinity of the AlN film. 

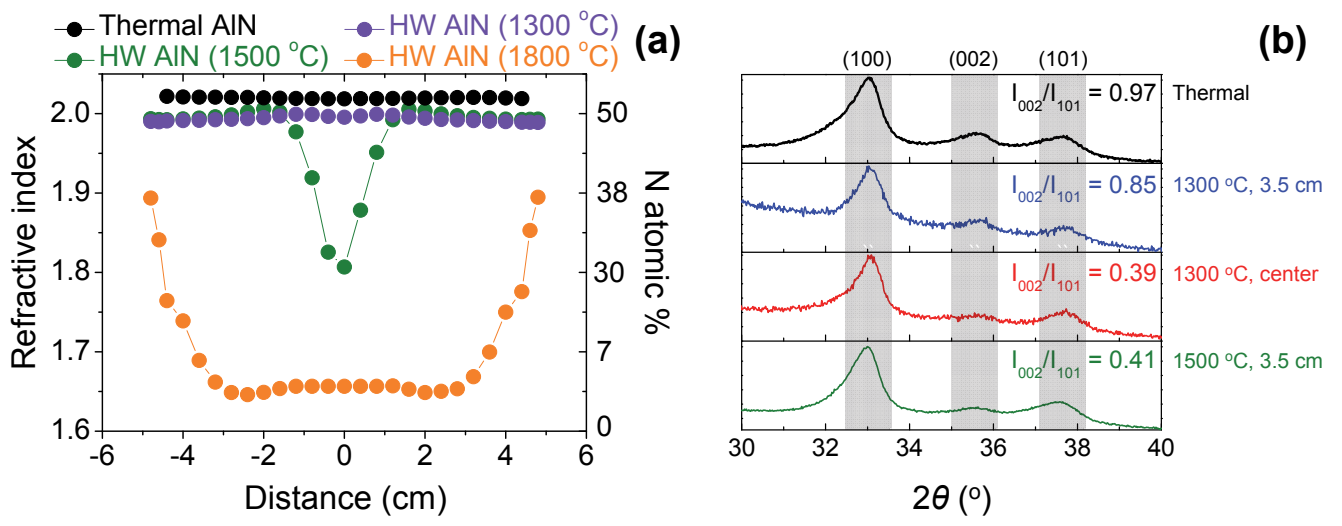

Fig. 3.8 (a) Variation of the refractive index and the oxynitride composition (N-content) with radial distance, at several filament temperatures. (b) Variation of the polycrystallinity (i.e., the preferential formation of the wurtztic (002) planes) of AlN films deposited thermally (in black), at $1300{ }^{\circ} \mathrm{C}$ and $1500{ }^{\circ} \mathrm{C}$ filament temperature and at $3.5 \mathrm{~cm}$ from wafer-center (in blue and green, resp.), and at $1300{ }^{\circ} \mathrm{C}$ filament temperature at the wafer-center (in red). All experiments were performed at 0.005 mbar pressure.

3.3.2.3 Effect of reactor pressure: The results of HWALD performed at different reactor pressures are similarly summarized in Fig. $3.9 \mathbf{a}$ and $\mathbf{~ b}$. As the reactor pressure is reduced to $0.005 \mathrm{mbar}$, the (at-H, O-containing) radicals are delivered over a larger area, due to an enhanced mean free path (in green). On the contrary, at a high pressure ( $0.5 \mathrm{mbar})$, the entirety of these radicals is confined within a narrow oxynitride ring (in cyan). GIXRD, performed on AlN formed beyond the ring, shows a clear effect of the reactor pressure on the $I_{002} / I_{101}$ ratio. The value increases from 0.03 to 0.70 for films deposited under 0.05 and 0.5 mbar, respectively, i.e., approaching that of thermal AlN. This observation is correlated to the recombination of the radicals $\left(\right.$ at $-\mathrm{H}, \mathrm{NH}_{\mathrm{x}}$ ) at the higher pressures.

The composition of the ring remains relatively independent of the pressure, unlike in the previous case of filament temperature. This implies, that even at 0.5 mbar there is an abundant supply of the O-containing radicals to the wafer-center. Further, as long as there is an abundance of these radicals, nitrogen incorporation hardly occurs at the center. However, once the O-containing radicals are entirely depleted, the nitride formation occurs. At all pressures, AlN films with low (1 - 2 at. \%) oxygen were deposited immediately outside the oxide ring (as verified by XPS). 


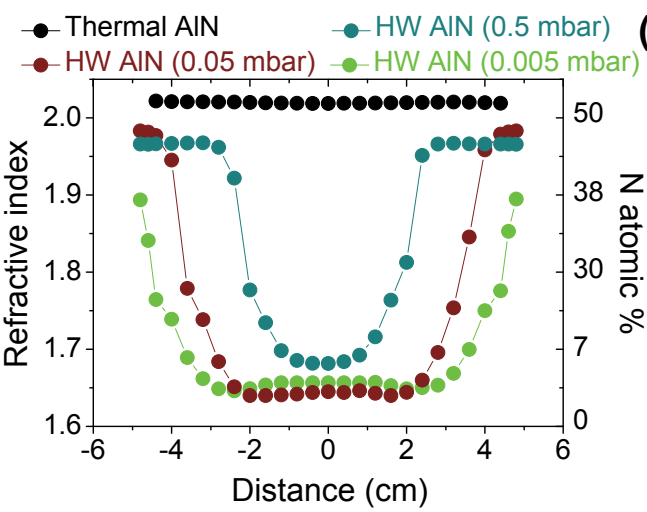

\section{(a)}

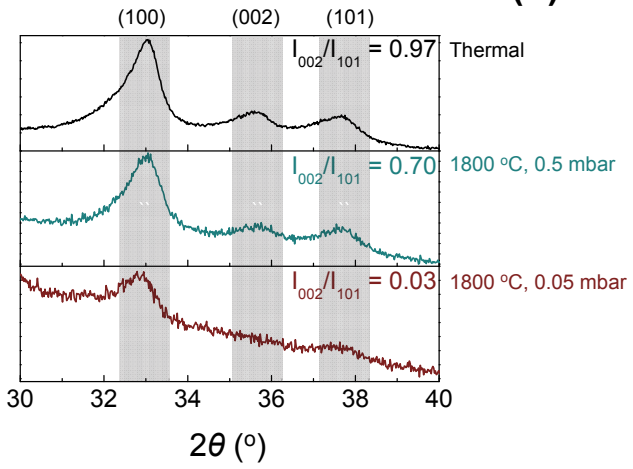

Fig. 3.9 (a) Variation of the refractive index and the oxynitride composition (N-content) with radial distance, at several reactor pressures. (b) Variation of the polycrystallinity of the samples, deposited thermally (in black), at 0.5 mbar pressure (in cyan) and at 0.05 mbar pressure (in maroon). All depostions were performed at $1800^{\circ} \mathrm{C}$ filament temperature.

3.3.2.4 Effect of removing sources of oxidants: The possible sources of oxidants in the reactor were identified to be: (i) $\mathrm{NH}_{3}$ and Ar contaminated with $\mathrm{O}_{2}$ and $\mathrm{H}_{2} \mathrm{O}$, (ii) the ceramic cap and stainless steel tubing of the HW stem, and (iii) the base pressure $\left(10^{-7} \mathrm{mbar}\right)$ of the reactor.

Installing $\mathrm{H}_{2} \mathrm{O}$ purifiers in both the $\mathrm{Ar}$ and $\mathrm{NH}_{3}$ lines, as well as removing the ceramic cap and tubing, hardly reduced the oxygen content. Further, to shorten the exposure time to the oxidants, reducing the Ar purge duration from $10 \mathrm{~s}$ (as used in all the previous experiments) to $3 \mathrm{~s}$ also did not prevent the formation of the oxynitride ring (Fig. 3.10). We therefore conclude that, unless the base pressure is lowered much below $10^{-7} \mathrm{mbar}$, it is not straight-forward to deposit O-free AIN films by HWALD, at a filament temperature higher than $1300^{\circ} \mathrm{C}$.

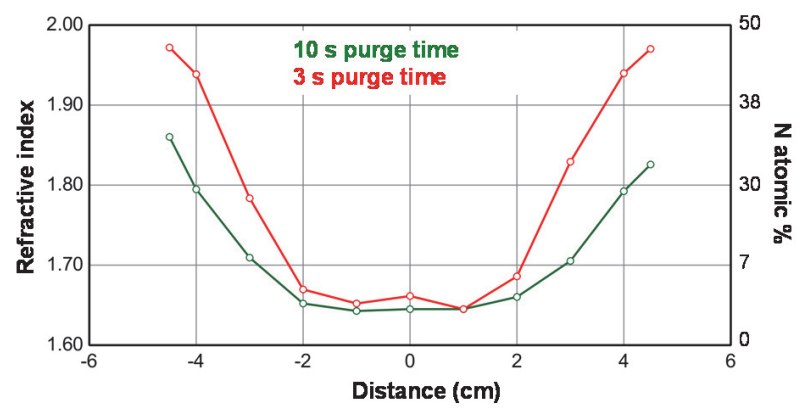

Fig. 3.10 Only a slight reduction of the oxynitride ring observed on reducing the purge time from $10 \mathrm{~s}$ to $3 \mathrm{~s}$. Both experiments were performed at the same filament temperature $\left(1800^{\circ} \mathrm{C}\right)$ and reactor pressure $\left(10^{-2} \mathrm{mbar}\right)$. 
3.3.2.5 Analysis of the chemical phases of the ring: To have an insight into the chemical phases of the films formed by HWALD, it was investigated whether the ring comprised of a single-phase Al-oxynitride or in fact, phase-segregated Al-oxide and Al-nitride. XPS was used for this purpose. The Al $2 p$ spectra from the ring from every sample could be fitted precisely with a single Gauss-Lorentz band. Rather, when more bands were introduced, e.g., one at the $\mathrm{BE}$ of $\mathrm{Al}_{2} \mathrm{O}_{3}$ and another at the $\mathrm{BE}$ of $\mathrm{AlN}$, the fit-error increased. The ability to fit the $\mathrm{Al} 2 \mathrm{p}$ spectra with a single band implies a single bonding environment in the layer, consistent with the formation of oxynitride of a single, homogeneous phase. The shift of the Al 2p BE with increasing O- (and decreasing N-contents) of the oxynitride layers is shown in Fig. 3.11.

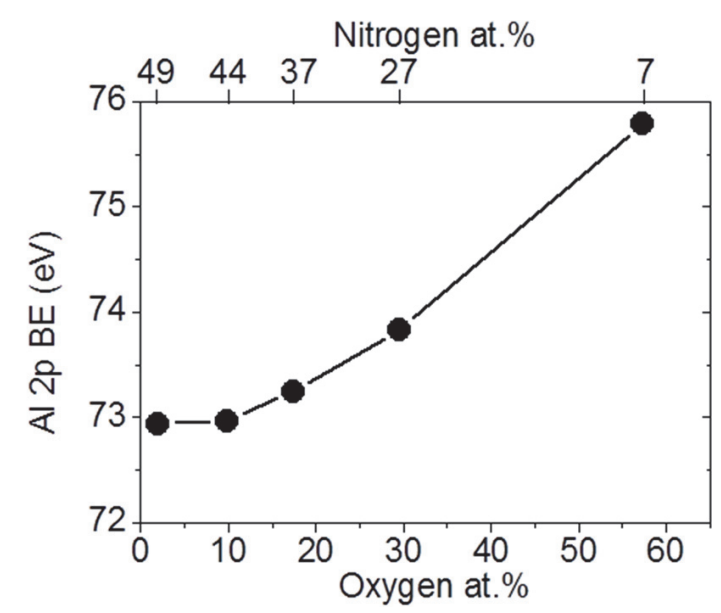

Fig. 3.11Measured shift of the $\mathrm{Al} 2 \mathrm{p}$ BE with changing the oxygen and nitrogen share of oxynitride. 


\subsection{Towards HWALD of GaN: Formation of Ga droplets}

In targeted HWALD of GaN from TMG and $\mathrm{NH}_{3}$ precursors, at- $\mathrm{H}$ similarly de-methylates the chemisorbed TMG into forming elemental Ga. However, instead of forming a thin film of $\mathrm{Ga}$ oxide or oxynitride, the de-methylated remains of TMG coalesce into micron-sized droplets of elemental Ga.

\subsubsection{Deposition outside the L-O-S position}

Depositions outside the L-O-S position (with the recipe $0.1 \mathrm{~s} \mathrm{TMG} \mathrm{/} 10 \mathrm{~s} \mathrm{Ar} /$ $4 \mathrm{~s} \mathrm{NH}_{3} / 10 \mathrm{~s} \mathrm{Ar}$, at $400{ }^{\circ} \mathrm{C}$ wafer temperature, $10^{-2}$ mbar reactor pressure, and $1500{ }^{\circ} \mathrm{C}$ filament temperature) resulted in the formation of a 'film' of milky-white appearance, under the location of the HW stem. Outside the stem, there was hardly any film formation (Fig. 3.12 a). Inspected with an optical microscope, the 'film' in fact revealed to consist of micron-sized droplets (Fig. 3.12 b). The composition of these droplets was confirmed as elemental Ga (perhaps bonded with hydrogen ${ }^{15}$ ) from EDX analysis (Fig. 3.12 c). Inspected visually, the density of these droplets was the highest at the center of the wafer, and decreased radially outwards, which correlates with the depletion of the at- $\mathrm{H}$ radicals. Changes to the ALD recipe (e.g., $\mathrm{NH}_{3}$ pulse time, Ar purge time and filament temperature) resulted in the formation such droplets; a GaN film was not formed at any condition.

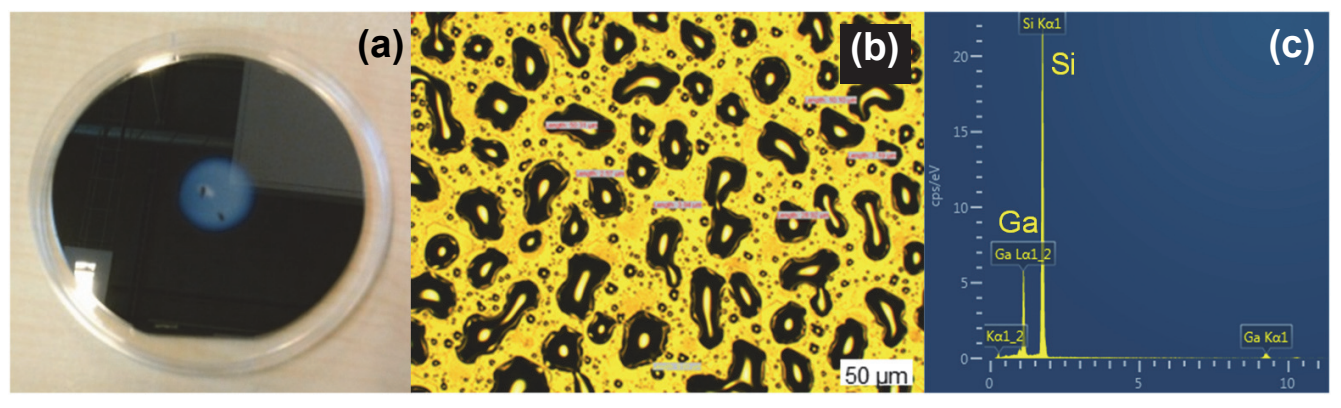

Fig. 3.12 (a) Milky deposition on the wafer under the stem, with HW outside the L-O-S, (b) Optical microscope image revealing the formation of micron-sized droplets composing the milky deposition, (c) EDX inspection of the above revealing the droplets to be elemental Ga. 

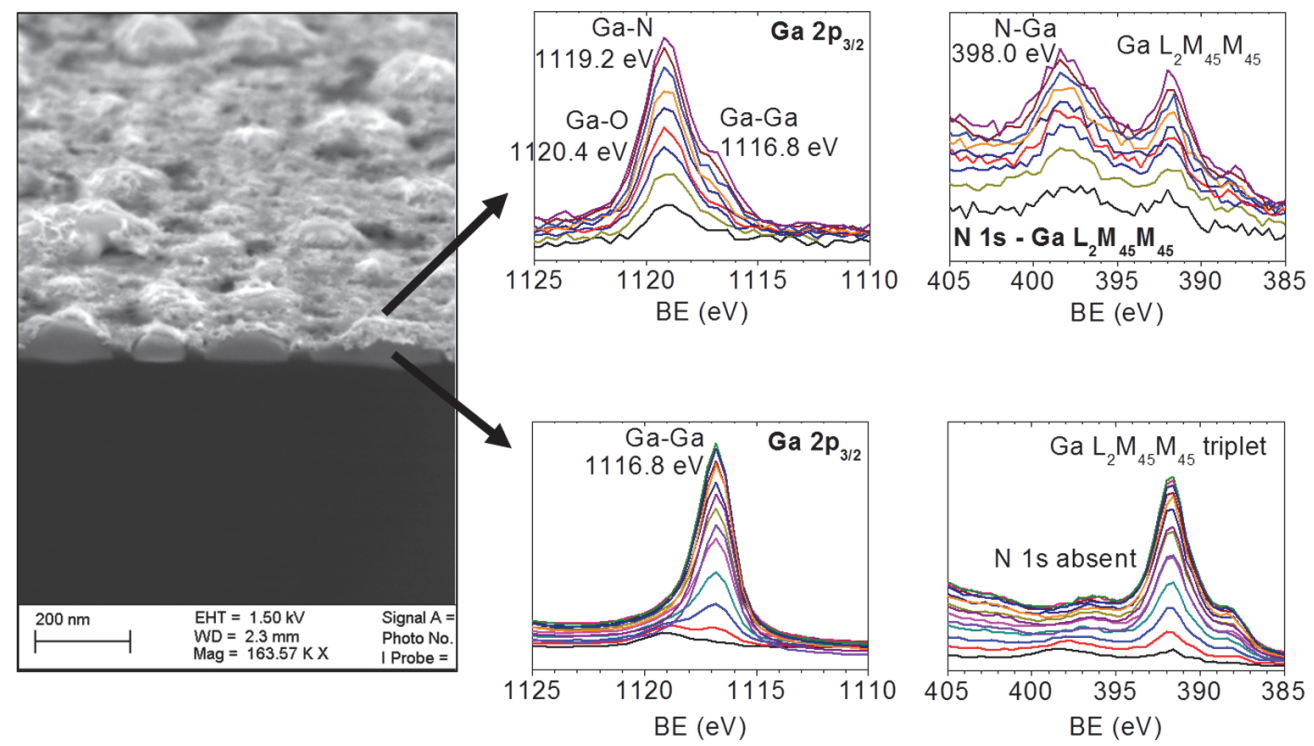

Fig. 3.13 (left) SEM image of the layer deposited with HW in the L-O-S position, showing the formation of droplets and a thin coating of droplets. (right) XPS from the two regions revealing Ga droplets and Ga-oxynitride film.

\subsubsection{Deposition in the L-O-S position}

In the L-O-S position, using the same recipe as above, initially there was the formation of droplets, followed by a thin film $(<10 \mathrm{~nm})$ coating of the droplets, as revealed from SEM (Fig. 3.13, left). XPS analysis (Fig. 3.13, right) revealed that these droplets were of elemental $\mathrm{Ga}$ (since only $\mathrm{Ga}-\mathrm{Ga}$ bonds, and no $\mathrm{Ga}-\mathrm{N}$ bonds were detected) and the coating was Ga-oxynitride ( since $\mathrm{Ga}-\mathrm{N}$ and $\mathrm{Ga}-\mathrm{O}$ bonds were both observed, followed by a minor amount of the $\mathrm{Ga}-\mathrm{Ga}$ bond). The formation of the droplets can be attributed to the demethylation of TMG by at-H. The occurrence of the droplets at the beginning of the ALD and thereafter the oxynitride coating, may have several explanations. One explanation is that, due to a high reactivity of at- $\mathrm{H}$ generated from the $\mathrm{NH}_{3}$, it first forms the Ga droplets on the substrate from demethylation of TMG (see Section 3.5). Thereafter, the delivery of the $\mathrm{NH}_{\mathrm{x}}$ radicals in the L-O-S position causes the nitridation of the droplets, although it is a slower reaction than the de-methylation. At the same time, the competing oxidation by O-containing radicals results in the oxynitride coating. At-H is presumably less reactive to the oxynitride compared to an intially-TMG-chemisorbed surface.

HWALD experiments were further performed at $10^{-2}$ mbar reactor pressure, at different filament temperatures. Whereas filament temperatures of 1500 and $1800{ }^{\circ} \mathrm{C}$ resulted 
only in the formation of Ga droplets, a temperature of $1300{ }^{\circ} \mathrm{C}$ resulted in the growth of a thin film, but with an extremely low GPC $\left(0.004 \mathrm{~nm} /\right.$ cycle). Increasing the $\mathrm{NH}_{3}$ pulse duration (from 2.5 to $10 \mathrm{~s}$ ) slightly improved the GPC (Fig. 3.14 a). The composition of the film was obtained from XPS (Fig. 3.14 b). Although $1300^{\circ} \mathrm{C}$ filament temperature does dissociate $\mathrm{NH}_{3}$ (confirmed by Te-etching and Si-nitridation), the amount of generated radicals is likely too low to cause reasonable GaN formation by the HW action. The extremely low GPC supports this assumption.
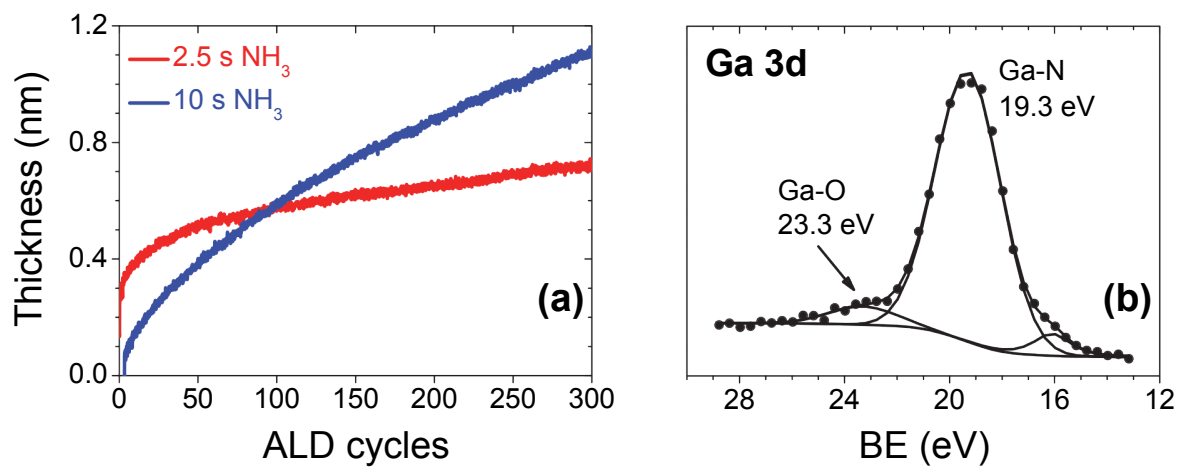

Fig. 3.14 (a) Thickness evolution of $\mathrm{GaN}$ deposited at $1300{ }^{\circ} \mathrm{C}$ filament temperature, under $2.5 \mathrm{~s}$ and $10 \mathrm{~S} \mathrm{NH}_{3}$ pulse durations, (b) Formation of $\mathrm{GaN}$ as verified from XPS.

\subsection{The action of at-H on chemisorbed TMA and TMG}

From the previous observations of the strong action of the at- $\mathrm{H}$ radicals (in demethylating chemisorbed TMA and TMG) and the insufficient action of the $\mathrm{NH}_{\mathrm{x}}$ radicals (in failing to nitridize the TMA and TMG) in the targeted HWALD of AlN and GaN films, we face the question: why is at-H significantly more reactive towards TMA and TMG than $N H_{x}$ radicals?

A possible answer may be found in the works of Yates et al. ${ }^{15-17}$. At-H, from both $\mathrm{H}_{2}$ and $\mathrm{NH}_{3}$ sources, de-methylates TMG with zero activation energy $\left(E_{\mathrm{A}}\right)$. This reaction is proposed to follow an Eley-Rideal (ER) mechanism. Indeed, at-H is prone to cause many reactions through this mechanism, and they follow a $0-E_{\mathrm{A}}$-route. For example, in the words of Rettner ${ }^{18}$ : "Most reactions are believed to occur by way of a Langmuir-Hinshelwood (LH) mechanism in which both reagents are considered to be chemisorbed and in thermal equilibrium with the surface. Certain highly reactive reactions are thought to proceed via an Eley-Rideal (ER) mechanism in which a reagent from the gas phase reacts directly on impact with a chemisorbed species". In this work, the author shows that at-H incident on deuterium (D), adsorbed on $\mathrm{Cu}(111)$ surface, forms HD species through the ER mechanism. The same 
author reports another reaction, involving at- $\mathrm{H}$ incident on $\mathrm{Cl}$, adsorbed on $\mathrm{Au}(111)$ surface, to also partially follow the ER mechanism ${ }^{19}$.

Not only the de-methylation of TMG, but also that of TMA may (partially) follow this mechanism. A theoretical study by Hiraoka et al. ${ }^{20}$ reports that the de-methylation of TMA by at-H occurs with an $E_{\mathrm{A}}$ of only $1.9 \mathrm{kcal} / \mathrm{mol}$. On the contrary, the nitridation of the de-methylated remains of TMA and TMG speculatively requires a higher $E_{\mathrm{a}}$. This explains the higher reactivity of at-H towards these metalorganic precursors than the $\mathrm{NH}_{\mathrm{x}}$ radicals. The implication is that, de-methylation of TMA and TMG readily occurs, whereas nitridation not. Practically, the fact that at-H is more reactive is important only if oxidants are present; otherwise, nitridation would still occur at a lower rate. The presence of oxidants causes the de-methylized TMA and TMG to form Al- and Ga-oxynitrides, respectively.

Considering the high reactivity of at- $\mathrm{H}$, a possible approach for $\mathrm{HW}$-assisted ALD of AlN and GaN would be to significantly increase the $\mathrm{NH}_{\mathrm{x}} /$ at- $\mathrm{H}$ ratio, in order to suppress the de-methylation. The presence of oxidants should also be minimized. This may be accomplished by using the HW stem from a material that has high recombination-probability $(r)$ of at-H and $\mathrm{O}$-containing radicals, and low $r$ of $\mathrm{NH}_{\mathrm{x}}$ radicals (our $\mathrm{HW}$ stem is constructed of alumina and stainless steel). Materials with high $r$ for at-H include $\mathrm{Si}(0.70)$, Ti (0.35) and $\mathrm{Cu}(0.14)$, whereas, that for O-containing radicals include stainless steel $(0.07)$ and cupric oxide (0.04). Stainless steel, however, has a low $r$ for at- $\mathrm{H}(0.032)$ and so is alumina $\left(1.8 \times 10^{-3}\right)$ and silica $\left(4 \times 10^{-5}\right)$. Silica also offers low $r$ for both the N- and O-containing radicals ( $3 \times 10^{-4}$ and $2 \times 10^{-4}$, respectively), whereas, stainless steel offers the most $\left(6 \times 10^{-3}\right)$ (see Knoops et al. and the references therein $\left.{ }^{21}\right)$. A careful choice among these candidates may be made, keeping in mind the specific chemistry of the deposition and their potential CMOS-compatibility. 


\subsection{Conclusions}

In this chapter, we introduced the novel HWALD technique for the deposition of AlN and GaN layers. The technique relied on the generation of radicals by catalytic dissociation of the $\mathrm{NH}_{3}$ precursor; the radicals then participating in ALD. To gain an insight into the mechanism of $\mathrm{HW}$ action, the dissociation of molecular $\mathrm{H}_{2}$ and $\mathrm{NH}_{3}$ and the delivery of atomic-H radicals were investigated, with the HW located outside and in the line-of-sight (L-O-S) positions. In either case, at-H could be efficiently delivered to the substrate, which was confirmed by in-situ monitoring of the etching of thin Te films. However, the $\mathrm{NH}_{\mathrm{x}}$ $(x=0-2)$ radicals generated by the $\mathrm{HW}$ dissociation of $\mathrm{NH}_{3}$ could only be delivered in the L-O-S position. This was confirmed by the nitridation of Si.

AlN and GaN depositions were attempted both outside and in the L-O-S positions. For AlN outside of the L-O-S position, pure Al-oxide was formed at the wafer-center, instead of AlN. In the L-O-S position, Al-oxynitride with only a low $\mathrm{N}$ content was formed. Radially outwards from the wafer-center, the O-content of the oxynitride gradually decreased (and the N-content increased), to ultimately form O-free AlN at the wafer-edges. The AlN was indeed formed by HW-action (and not thermally), since it had different polycrystallinity than thermally-deposited AlN. The change in poly-crystallinity was apparent from a lower preferential formation of the (002) wurtzitic crystal planes of the HW-AlN. On the contrary, during targeted GaN depositions, there was the formation of Ga droplets outside the L-O-S position. In the L-O-S position, formation of Ga droplets was followed by an oxynitride film.

The occurrence of Al-oxide and elemental-Ga was ascribed to the significantly higher reactivity of atomic- $\mathrm{H}$ compared to $\mathrm{NH}_{\mathrm{x}}$ radicals, and the resulting de-methylation (instead of nitridation) of TMA and TMG, followed by oxidation of the de-methylated surface by residual oxidants. For successful HW ALD of AlN, reducing the amount of residual oxidants in the reactor is crucial. To proceed with HW ALD of GaN, reducing the relative proportion of at- $\mathrm{H}$ to $\mathrm{NH}_{\mathrm{x}}$ radicals can be of importance. These may be accomplished by an alternative choice of the HW stem. 


\section{References}

1. A. Y. Kovalgin and A. A. I. Aarnink, U.S. Patent App. 13/918,094, 2017.

2. G. Yuan, H. Shimizu, T. Momose and Y. Shimogaki, Journal of Vacuum Science \& Technology A: Vacuum, Surfaces, and Films, 2014, 32, 01 A104.

3. M. Yang, A. A. I. Aarnink, J. Schmitz and A. Y. Kovalgin, Thin Solid Films, 2018, 649, $17-$ 23.

4. M. Yang, A. A. I. Aarnink, J. Schmitz and A. Y. Kovalgin, Thin Solid Films, 2018, 646, 199208.

5. J. Sumakeris, Z. Sitar, K. Ailey-Trent, K. More and R. Davis, Thin Solid Films, 1993, 225, 244-249.

6. M. Yang, A. A. I. Aarnink, A. Y. Kovalgin, R. A. M. Wolters and J. Schmitz, Physica Status Solidi (A), 2015, 212, 1607-1614.

7. J. N. Smith Jr and W. L. Fite, The Journal of Chemical Physics, 1962, 37, 898-904.

8. H. Wise and C. M. Ablow, The Journal of Chemical Physics, 1961, 35, 10-18.

9. D. A. Outka, Surface Science, 1990, 235, L311-L318.

10. H. Umemoto, K. Ohara, D. Morita, T. Morimoto, M. Yamawaki, A. Masuda and H. Matsumura, Japanese Journal of Applied Physics, 2003, 42, 5315.

11. Y. Shi, B. Eustergerling and X. Li, Thin Solid Films, 2008, 516, 506-510.

12. A. Y. Kovalgin, M. Yang, S. Banerjee, R. O. Apaydin, A. A. I. Aarnink, S. Kinge and R. A. M. Wolters, Advanced Materials Interfaces, 2017, 4, 1700058.

13. A. Izumi, Thin Solid Films, 2006, 501, 157-159.

14. S. M. Gates, R. R. Kunz and C. M. Greenlief, Surface Science, 1989, 207, 364-384.

15. D. B. Mawhinney, J. A. Glass Jr and J. T. Yates Jr, Journal of Vacuum Science \& Technology A: Vacuum, Surfaces, and Films, 1999, 17, 679-685.

16. S. Lucas, W. Partlow, W. Choyke and J. Yates Jr, Journal of Vacuum Science \& Technology A: Vacuum, Surfaces, and Films, 1994, 12, 3040-3047.

17. A. Hubner, S. Lucas, W. Partlow, W. Choyke, J. Schaefer and J. Yates, Journal of Vacuum Science \& Technology A: Vacuum, Surfaces, and Films, 1995, 13, 1831-1836.

18. C. Rettner, Physical Review Letters, 1992, 69, 383.

19. C. T. Rettner and D. J. Auerbach, Science, 1994, 263, 365-367.

20. Y. S. Hiraoka and M. Mashita, Japanese Journal of Applied Physics, 1992, 31, 3703. 
Chapter 3

21. H. Knoops, E. Langereis, M. Van De Sanden and W. Kessels, Journal of The Electrochemical Society, 2010, 157, G241-G249. 


\section{Appendix 3.1: Dependence of the refractive index on oxynitride composition}

A comparison between the composition of the Al-oxynitride $\left(\mathrm{AlO}_{\mathrm{x}} \mathrm{N}_{\mathrm{Y}}\right)$ layers, obtained from XPS and SE analyses, is presented in Fig. A 3.1. XPS is certainly the more accurate means of determining the composition, since SE solely relies on changes in the optical constants with the composition. To construct the optical model, it was assumed that the oxynitride was a mixture of $\mathrm{Al}_{2} \mathrm{O}_{3}$ and $\mathrm{AlN}$, and an effective medium approximation was thereafter used. However, the oxynitride is of single-phase (Section 3.3.2.5), which perhaps causes the difference in compositions obtained from the two techniques (Fig. A 3.1). Still, the figure can serve in estimating the oxynitride composition directly from SE (without having to resort to XPS).

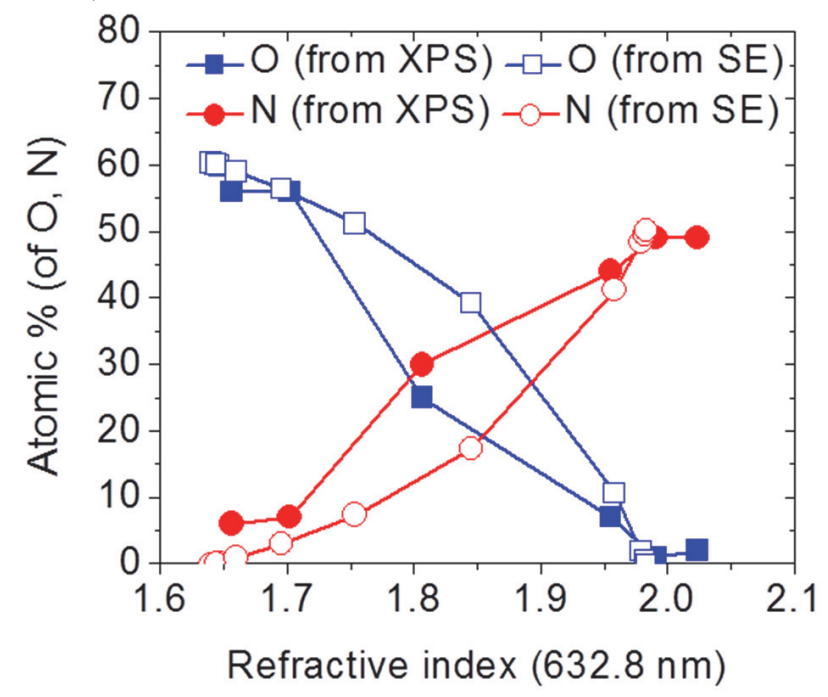

Fig. A 3.1 Relation between the oxynitride compostion ( $\mathrm{O}$ and $\mathrm{N}$ in atomic \%) and the refractive index. The refractive index is obtained by SE. The composition is obtained by SE (open symbols) and XPS (solid symbols). 


\section{Appendix 3.2: Additional characterization of the reactor by at-H}

A 3.2.1 On determining the true reactor pressure: In an ALD reactor, it is crucial that the excess (unreacted) precursors and the reaction by-products leave the reactor during the purge times. Purge-holes are present for this purpose. However, a non-optimal geometry or size of the holes may reduce their throughput. Therefore, the reactor is not always sufficiently purged and significant amount of CVD reactions may set in. Moreover, while designing an experimental home-built reactor, it may not always be possible to pre-determine the number of purge-holes required for an efficient purging. Such may result in the accumulation of gases inside the reactor during ALD, thereby increasing the internal pressure. In many reactor designs (ours included), the pressure gauges are not located directly at the site of the substrate, but at some distance away. This makes it non-trivial to determine the true pressure under which the film-forming reactions occur. Te etch tests can be performed in such situations.

Fig. A 3.2 a shows the Te etching kinetics by at-H in time. At-H is generated from $\mathrm{H}_{2}$. The etch-rate is much lower than expected (e.g., compare with Fig. 3.3 a, red), and the etching stops completely within $30 \mathrm{~min}$. This can be explained from the recombination of at- $\mathrm{H}$ at increased reactor pressures from the continued introduction of $\mathrm{H}_{2}$ and its inefficient purging, causing a local pressure increase. The inefficient purging was ascribed to a lower throughput of the purge-holes (inspection revealed particles deposited in them). Another reason behind decrease in the etch-rate is the oxidation of the Te film, although this effect is less severe than that shown in Fig. A 3.2 a.
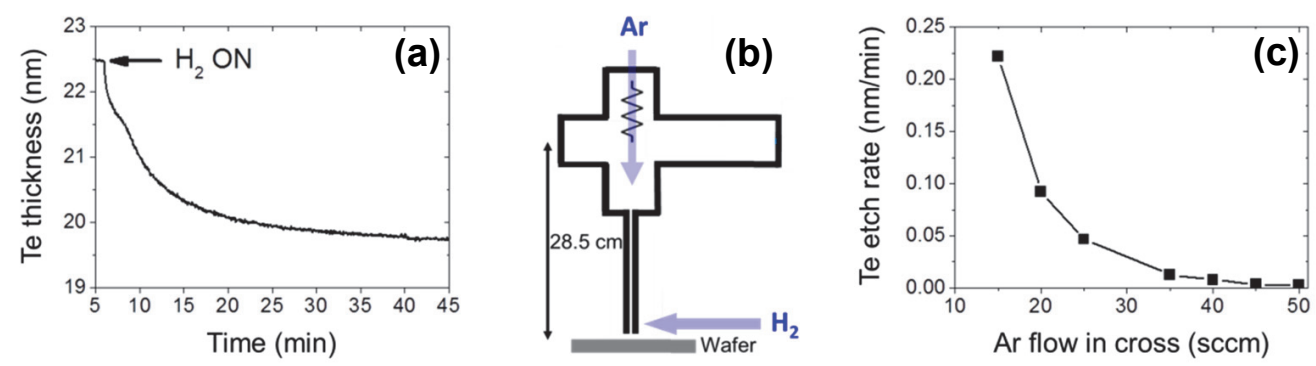

Fig. A 3.2 (a) Gradual stopping of Te etch due to pressure build-up inside the reactor. The pressure gauge reading during the experiment was $3 \times 10^{-3}$ mbar, (b) The schematic used for BSD tests, and (c) the variation of Te etch rate with Ar flow, at a constant $\mathrm{H}_{2}$ flow of $50 \mathrm{sccm}$.

\section{A 3.2.2 Testing for the occurrence of backstream diffusion of the precursors towards the} $\boldsymbol{H W}$ : At the low pressures used in AIN and GaN HWALD $\left(10^{-3}-10^{-2} \mathrm{mbar}\right)$, the mean free path can be as high as few centimeters. Therefore it is necessary to test and prevent the occurrence of backstream diffusion (BSD) of the TMA and TMG precursors towards the 
HW (which are introduced laterally, recall Fig. 3.2 a, b). Otherwise the HW would get coated with substances such as carbon and gallium. Besides, the efficient purging of the excess precursors would also be hardly achievable.

The occurrence of BSD was tested by introducing $\mathrm{H}_{2}$ laterally through the original TMG inlets (refer to Fig. A 3.2 b for the schematic), at a constant flow rate (50 sccm) under a low reactor pressure $\left(10^{-3} \mathrm{mbar}\right)$, to optimally promote its BSD. Ar was continuously flowed through the stem, as shown. The variation of the Te etch rate with Ar flow (Fig. A 3.2 c) reveals that beyond a threshold Ar flow rate $(50 \mathrm{sccm})$, no Te etching occurs. This experiment gives an indication of the precursor and carrier gas flow rates that must be used in HWALD experiments of AIN and GaN films, to avoid the effects of BSD. 


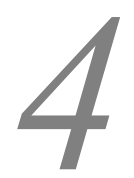

\section{Thermal ALD of composite} GaN-C-Ga ('GaCN') layers"

${ }^{*}$ This chapter is based on:

S. Banerjee, A. J. Onnink, S. Dutta, A. A. I. Aarnink, D. J. Gravesteijn and A. Y. Kovalgin, The Journal of Physical Chemistry C, 2018, 122(51), 29567-29576. 


\subsection{Introduction}

In this chapter, we study novel composite $\mathrm{GaCN}$ layers comprising nanoscale clusters of polycrystalline wurtzitic $\mathrm{GaN}$, with $\mathrm{Ga}$ and $\mathrm{C}$ inclusions. The layers are deposited by alternating pulses of trimethylgallium (TMG) and ammonia $\left(\mathrm{NH}_{3}\right)$ on $\mathrm{Si}$ substrates, under a range of temperatures and ammonia partial pressures. These two conditions determine the layers' composition. Since $\mathrm{GaCN}$ had been hardly reported in the literature, a thorough material investigation is performed to determine whether the material is single-phase or segregated into multiple phases. The latter is revealed to be true. Even though phasesegregated, the carbon (C) content profoundly influences the layers' optical properties. Varying the C-content between 0 to 10 atomic \%, the refractive index can be tuned from 2.20 to 2.46 , at $1500 \mathrm{~nm}$ wavelength. The variation of the layer composition with temperature and pressure not only allows to tailor the optical properties, but also provides insights into the growth mechanism. This, in turn, opens up the route to realize $\mathrm{GaCN}$ layers with a wide range of compositions. The layers, although composite in nature, are regarded as the Gaanalogue to other known carbonitrides such as $\mathrm{BCN}$ and $\mathrm{CN}$.

The lack of reports on $\mathrm{GaCN}$, unlike $\mathrm{BCN}$ or $\mathrm{CN}$, is perhaps because in the development of gallium nitride-based electronics, the attention has been focussed towards realizing stoichiometric, crystalline layers to serve the requirements of the opto-electronics industry. Carbon has been occasionally used as a dopant, and higher concentrations have been shown to affect the device performance ${ }^{1}$. A second reason is speculated to be the intrinsically unstable nature of gallium carbide ${ }^{2}$, prohibiting its use as a stand-alone material. This limits the prospect of the preparation of $\mathrm{GaCN}$ from it. However, in terms of the potential applications of $\mathrm{GaCN}$ (one such is discussed in Appendix 4), research on this novel composite should not be ignored.

This chapter is divided into two broad sections: Section $\mathbf{4 . 2}$ addresses the characterization of $\mathrm{GaCN}$ layers, and Section 4.3 discusses its growth kinetics. More specifically, Section 4.2.1 describes how these layers were prepared. Section 4.2.2 reports their characterization using several techniques, with the aim of studying whether the layers are single-phase or phase-segregated. Section 4.2.3 describes the optical properties of the $\mathrm{GaCN}$ composite. Section 4.3.1 and 4.3.2 addresses the growth mechanism of the composite, proposing the existence of a novel chemical complex that is speculated to play an important role in $\mathrm{GaCN}$ growth. Section $\mathbf{4 . 4}$ concludes the chapter. 


\subsection{GaCN deposition and characterization}

\subsubsection{Layer cross section and morphology}

GaCN layers*, with thicknesses varying between 7 and $110 \mathrm{~nm}$, were deposited on 4-inch Si substrates from alternate pulses of $\mathrm{TMG}$ and $\mathrm{NH}_{3}$ with in-between $\mathrm{Ar}$ purges, mimicking an ALD processes (see caption to Fig. 4.1 for the recipe). The depositions were performed between 400 and $600{ }^{\circ} \mathrm{C}$ and at a range of reactor pressures between 0.01 and 10 mbar. The corresponding $\mathrm{NH}_{3}$ partial pressures $\left(P_{\mathrm{NH} 3}\right)$ varied between $0.7 \times 10^{-3}$ and 7.25 mbar. An ALD AIN layer $(25 \mathrm{~nm}$ ) was first in-situ deposited on Si as buffer layer. To grow the $\mathrm{GaCN}$ layers, a buffer layer is not necessary per se, and growth can be accomplished directly on $\mathrm{Si}$ or $\mathrm{SiO}_{2}$ substrates.

The layers were analysed using electron microscopy. Fig. 4.1 a shows the crosssectional energy selective backscattered (ESB) image of a representative GaCN layer. Fig. 4.1 b shows the plan-view InLens scanning electron microscope (SEM) image of the same. A grainy surface is revealed with an average grain size of $73 \mathrm{~nm}$.
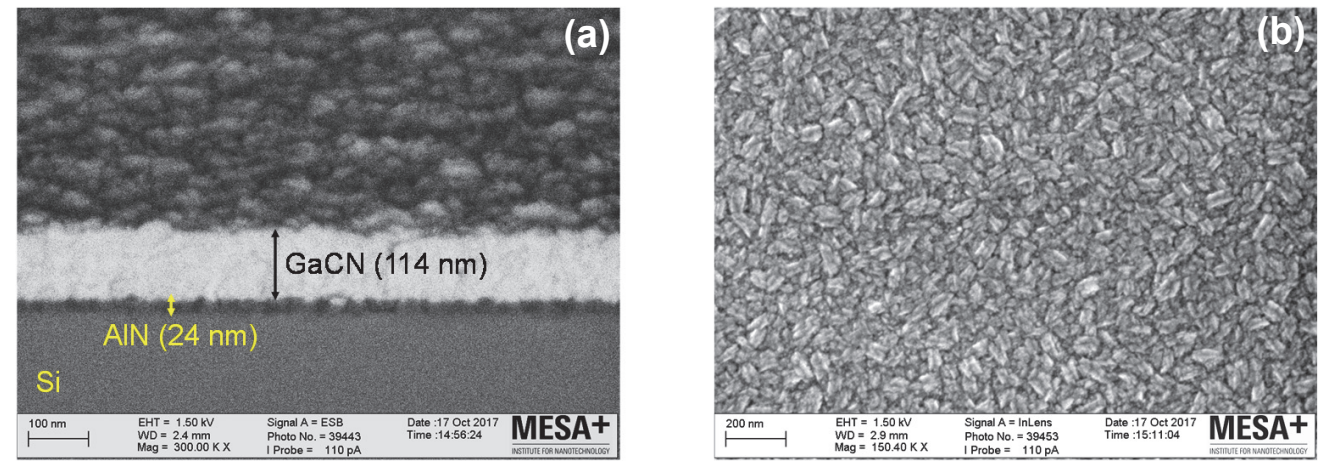

Fig. 4.1 (a) Cross-section ESB, (b) plan-view SEM image of a representative $\mathrm{GaCN}$ layer. The $\mathrm{GaCN}$ was deposited using the recipe: $0.1 \mathrm{~s} \mathrm{TMG} / 2 \mathrm{~s} \mathrm{Ar} / 1 \mathrm{~s} \mathrm{NH} / 4 \mathrm{~s} \mathrm{Ar}$, at $550{ }^{\circ} \mathrm{C}$ and $10^{-1} \mathrm{mbar} P_{\mathrm{NH} 3}$. The AlN buffer layer was deposited with: $0.5 \mathrm{~s}$ TMA / $4 \mathrm{~s} \mathrm{Ar} / 2.5 \mathrm{~s} \mathrm{NH} / 4 \mathrm{~s} \mathrm{Ar}$, at $350{ }^{\circ} \mathrm{C}$ and $10^{-3}$ mbar $P_{\mathrm{NH} 3}$.

\footnotetext{
* The abbreviation ' $\mathrm{GaCN}$ ' qualitatively reflects the elemental composition, specifying neither the layer stoichiometry nor the existence of single or multiple phases. Section 4.2.2 describes the characterization techniques that reveal the layers to be a composite, consisting of multiple phases segregated at the nanoscale.
} 


\subsubsection{Chemical bonding and phase-segregation analyses}

4.2.2.1 FTIR analysis: FTIR analysis was done in the transmission mode in the 400 to $4000 \mathrm{~cm}^{-1}$ spectral range. Fig. 4.2 shows the spectra of a representative $\mathrm{GaCN}$ layer (115 nm thickness) (in red) and compares it with an ALD GaN layer (23 nm) (in blue); the latter is used as a reference. Table 4.1 lists the various bonds identified in the two layers. The details on the preparation and characterization of the reference ALD GaN layer are presented in Chapter 5.

Both $\mathrm{GaCN}$ and $\mathrm{GaN}$ layers show strong absorption at $530 \mathrm{~cm}^{-1}$, which corresponds to the $\mathrm{Ga}-\mathrm{N}$ stretching vibration in wurtzitic $\mathrm{GaN}$ crystals ${ }^{3}$. A series of $\mathrm{GaCN}$ samples with different compositions was prepared for this work. Irrespective of their composition, all the samples exhibited absorption at this wavenumber. Whereas for the reference GaN sample this absorption is expected, its constant presence in the $\mathrm{GaCN}$ layers implies the occurrence of $\mathrm{Ga}-\mathrm{N}$ bonds in the layers. Further analyses will show that these bonds originate from nanoscale wurtzitic $\mathrm{GaN}$ poly-crystals in $\mathrm{GaCN}$.

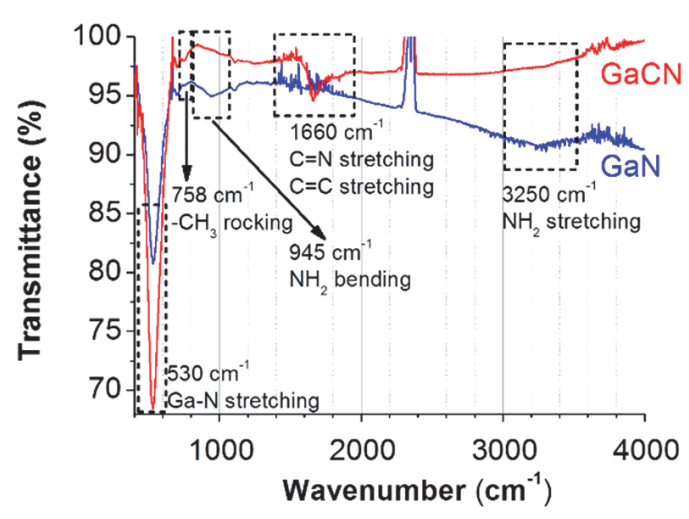

Table 4.1 Summary of the chemical bonds in $\mathrm{GaN}$ and $\mathrm{GaCN}$.

\begin{tabular}{|c|c|c|c|}
\hline $\begin{array}{c}\text { Wavenumber } \\
\left(\mathbf{c m}^{-1}\right)\end{array}$ & Bond & GaN & GaCN \\
\hline 530 & $\mathrm{Ga}-\mathrm{N}$ & $\checkmark$ & $\checkmark$ \\
\hline 758 & $\mathrm{Ga}-\mathrm{CH}_{3}$ & X & $\checkmark$ \\
\hline 945 & $-\mathrm{NH}_{2}$ & $\checkmark$ & $\times$ \\
\hline 1660 & $\mathrm{C}=\mathrm{C}, \mathrm{C}=\mathrm{N}$ & $\mathrm{X}$ & $\checkmark$ \\
\hline 1840 & $\mathrm{C}=\mathrm{C}$ & $\mathrm{X}$ & $\checkmark$ \\
\hline 3250 & $-\mathrm{NH}_{2}$ & $\checkmark$ & $X$ \\
\hline
\end{tabular}

Fig. 4.2 FTIR spectra comparing a representative GaCN layer with a reference ALD GaN layer.

The GaCN sample shows an absorption at $1660 \mathrm{~cm}^{-1}$ which is not present in $\mathrm{GaN}$. Some possible candidates for this absorption are (i) deformation of $\mathrm{sp}^{3}$-hybridized $\mathrm{N}-\mathrm{H}$ bonds, (ii) $\mathrm{sp}^{2}$-hybridized $\mathrm{C}=\mathrm{N}$ stretching vibration, and (iii) $\mathrm{sp}^{2}$-hybridized $\mathrm{C}=\mathrm{C}$ stretching vibration ${ }^{4}$. The GaN sample instead shows an absorption at $945 \mathrm{~cm}^{-1}$ corresponding to $\mathrm{sp}^{3}$ hybridized $-\mathrm{NH}_{2}$ bending mode ${ }^{5}$, which is not exhibited by GaCN. Similarly, the absorption at $3250 \mathrm{~cm}^{-1}$ from the $-\mathrm{NH}_{2}$ stretching mode ${ }^{4}$ is only present in $\mathrm{GaN}$. Therefore, the presence of $\mathrm{sp}^{3}$-hybridized $\mathrm{N}-\mathrm{H}$ bonds in $\mathrm{GaCN}$ may be neglected.

The remaining candidates for the $1660 \mathrm{~cm}^{-1}$ absorption are $\mathrm{sp}^{2}$-hybridized $\mathrm{C}=\mathrm{N}$ and $\mathrm{C}=\mathrm{C}$ bonds. The $\mathrm{sp}^{2} \mathrm{C}=\mathrm{C}$ stretching mode is reported to have its fundamental absorption at 
$1660 \mathrm{~cm}^{-1}$ and an overtone absorption between $1800-1850 \mathrm{~cm}^{-1}(4)$. The overtone absorption is detected in $\mathrm{GaCN}$ at $1840 \mathrm{~cm}^{-1}$. As the XPS results (next section) will reveal, the relative share of $\mathrm{C}=\mathrm{N}$ to $\mathrm{C}=\mathrm{C}$ bonds is high in $\mathrm{GaCN}$.

In $\mathrm{GaCN}$, carbon can be additionally present as incompletely dissociated TMG residues. These so-called 'fragments' constitute $\mathrm{Ga}-\left(\mathrm{CH}_{3}\right)_{2}$ (i.e., dimethylgallium or DMG) and $\mathrm{Ga}-\mathrm{CH}_{3}$ (i.e., monomethylgallium or $\mathrm{MMG}$ ). In these species, the $-\mathrm{CH}_{3}$ rocking vibration is reported at $746 \mathrm{~cm}^{-1(6)}$. The absorption at $758 \mathrm{~cm}^{-1}$ in $\mathrm{GaCN}$ can therefore be attributed to the fragments. The absorption of $\mathrm{C}-\mathrm{Ga}$ bonds is reported at $565 \mathrm{~cm}^{-1(6)}$, which is submerged under the strong $\mathrm{Ga}-\mathrm{N}$ absorption.

4.2.2.2 XPS analysis: To further discern the bonding environment in $\mathrm{GaCN}$, depth profiled XPS was performed using an Al K $\alpha$ monochromatic X-ray source. The Ga 2p $3 / 2$, N 1s, and $\mathrm{C}$ 1s photoelectron spectra were analysed. Using the hydrocarbon contamination of the surface from the ambient, the surface $\mathrm{C} 1 \mathrm{~s}$ peak position was calibrated at $284.8 \mathrm{eV}^{7}$. Sputter depth profiling was performed using $2 \mathrm{kV} \mathrm{Ar}{ }^{+}$ions. The spectra were deconvoluted by Gaussian-Lorentzian bands, assuming an iterated-Shirley background. The depth profiling removed the surface hydrocarbon contamination after the first sputter, and the analysis of the bonding environment was done on the elemental spectra inside the $\mathrm{GaCN}$ layer.
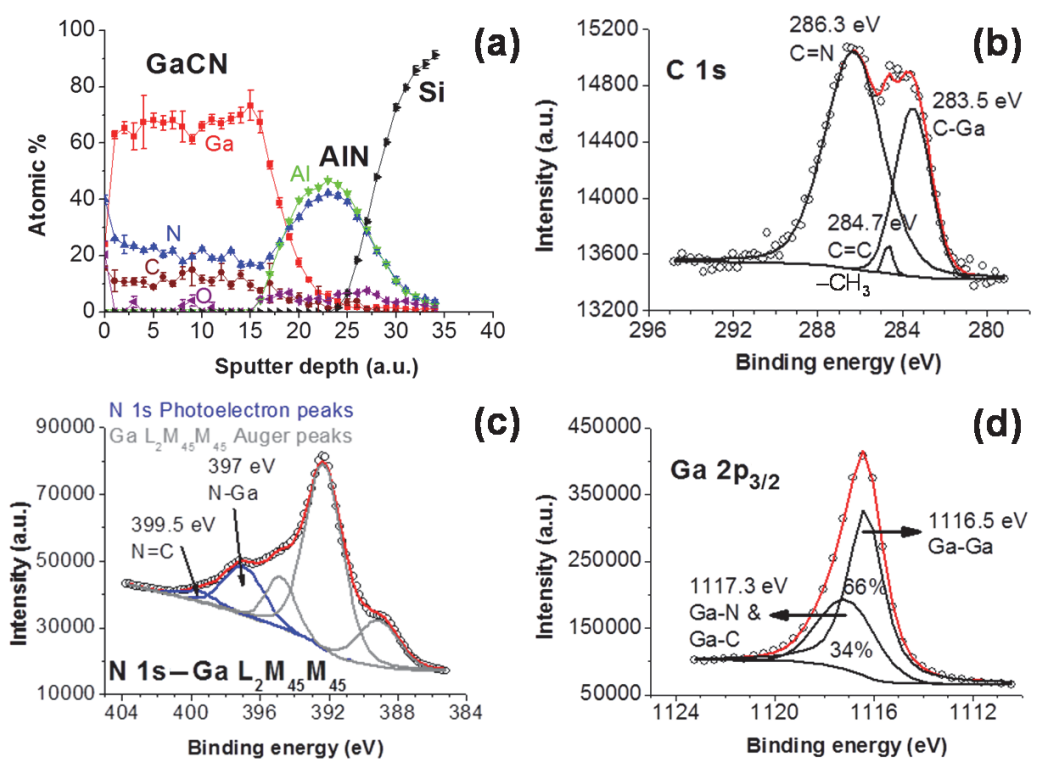

(c)

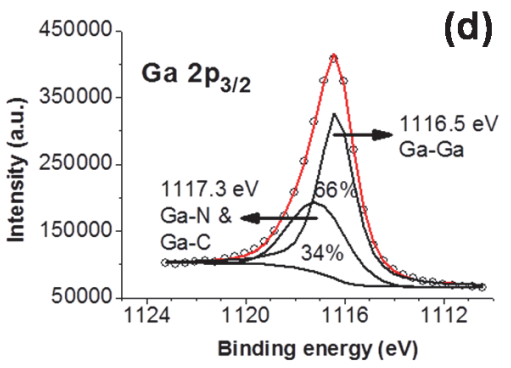

Fig. 4.3 XPS analyses showing (a) Depth profiled composition of a GaCN/AlN/Si stack. The deconvolution of (b) photoelectron $\mathrm{C}$ 1s spectrum, (c) photoelectron N 1s and Auger $\mathrm{Ga}_{2} \mathrm{M}_{45} \mathrm{M}_{45}$ spectra, and (d) photoelectron $\mathrm{Ga} 2 \mathrm{p}_{3 / 2}$ spectrum, revealing the various chemical bonds. 
A. Depth profiled composition. Fig. 4.3 a shows the depth profiled composition of the representative $\mathrm{GaCN}$ layer (deposited on $\mathrm{AlN}$ buffer layer) on $\mathrm{Si}$. A high Ga content, compared to $\mathrm{N}$ and $\mathrm{C}$ contents, is revealed. The $\mathrm{C}$ content is rather uniform throughout the $\mathrm{GaCN}$ thickness. However, a slight opposite gradient in the Ga and $\mathrm{N}$ contents is observed (the former decreasing towards the surface and the latter increasing), the cause of which is not clearly understood. The layer has an average composition of $\mathrm{Ga}_{0.66} \mathrm{C}_{0.10} \mathrm{~N}_{0.24}$.

B. The C 1s spectrum. The C 1s photoelectron spectrum is shown in Fig. 4.3 b. The shape suggests multiple bonding environments of carbon. The spectrum was deconvoluted by three Gaussian-Lorentzian bands at binding energies (BE) of 286.3, 284.7 and $283.5 \mathrm{eV}$.

The $286.3 \mathrm{eV}$ band corresponds precisely with that of $\mathrm{sp}^{2}$-hybridized $\mathrm{C}=\mathrm{N}$ bonds, reported in other carbonitrides such as $\mathrm{BCN}$ and $\mathrm{CN}^{8-9}$. This finding supports the previous attribution of the $1660 \mathrm{~cm}^{-1}$ FTIR band to $\mathrm{sp}^{2} \mathrm{C}=\mathrm{N}$. The other FTIR candidate, i.e., the $\mathrm{sp}^{2}$ $\mathrm{C}=\mathrm{C}$ band, has its XPS signature between 284.2 and $284.6 \mathrm{eV}^{7}$. The band at $284.7 \mathrm{eV}$ is expected to be the signature, which suggests that $\mathrm{sp}^{2} \mathrm{C}=\mathrm{C}$ bonds are present only in small amounts in $\mathrm{GaCN}$.

The $283.5 \mathrm{eV}$ band has an excellent agreement with $\mathrm{C}-\mathrm{Ga}$ bonding in TMG and its dissociated fragments such as DMG and $\mathrm{MMG}^{10-11}$. The $-\mathrm{CH}_{3}$ groups of such fragments are also reported to have their XPS signature around $284.7 \mathrm{eV}^{7}$. However, a much-stronger signature of $\mathrm{C}-\mathrm{Ga}$, compared to $-\mathrm{CH}_{3}$, is obtained.

C. The $\mathbf{N} 1$ s spectrum. The N 1s photoelectron spectrum is shown in Fig. 4.3 c. It partly overlaps with the Ga Auger $\mathrm{L}_{2} \mathrm{M}_{45} \mathrm{M}_{45}$ triplet due to the energy of the $\mathrm{Al} k \alpha \mathrm{X}$-ray source ${ }^{12}$ (see Appendix 2.1). The deconvolution reveals the photoelectron N 1s spectrum (in blue) and the Auger $\mathrm{Ga} \mathrm{L}_{2} \mathrm{M}_{45} \mathrm{M}_{45}$ triplet spectra (in grey). In this $\mathrm{GaCN}$ sample, and all the others, the Auger bands were noticeably stronger than the $\mathrm{N} \mathrm{1s}$ band. This is due to the high Ga content in the GaCN. For comparison, the $\mathrm{N} 1 \mathrm{~s}-\mathrm{Ga}_{2} \mathrm{M}_{45} \mathrm{M}_{45}$ complex and its deconvolution from the reference $\mathrm{GaN}$ layer is shown in Fig. 4.4 a. Due to the similar (stoichiometric) amounts of $\mathrm{Ga}$ and $\mathrm{N}$ in the reference GaN layer, deconvolution of the Auger spectra into a triplet is not straightforward. A doublet Auger spectra, instead of triplet, was therefore used.

The $\mathrm{BE}$ of the $\mathrm{N} 1 \mathrm{~s}$ band at $397.0 \mathrm{eV}$ in the $\mathrm{GaCN}$ layer (Fig. $4.3 \mathrm{c}$ ) and at $396.8 \mathrm{eV}$ in the reference GaN layer (Fig. 4.4 a) are both in excellent agreement with the $\mathrm{N}-\mathrm{Ga} \mathrm{BE}$ of $\mathrm{GaN}$ films, at $397.1 \mathrm{eV}^{13}$ and $396.9 \mathrm{eV}^{14}$. This indicates the possible existence of chemicallynon-interacting $\mathrm{GaN}$ inclusions in $\mathrm{GaCN}$, which is also in agreement with the FTIR observation of the $530 \mathrm{~cm}^{-1}$ band. 

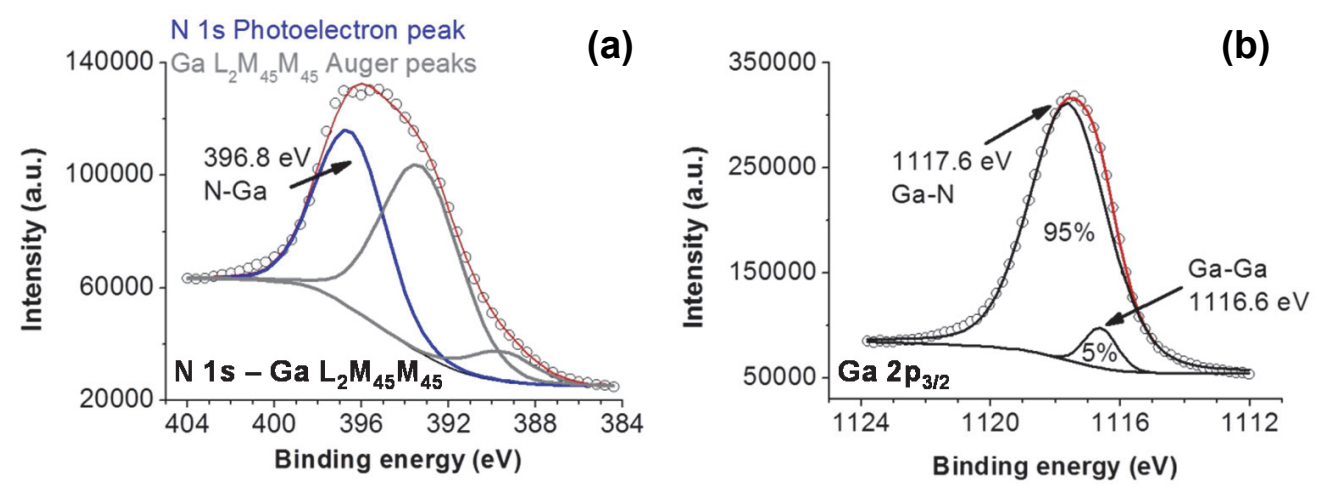

Fig. 4.4 Deconvolution of the (a) photoelectron $\mathrm{N} 1 \mathrm{~s}-$ Auger $\mathrm{GaL}_{2} \mathrm{M}_{45} \mathrm{M}_{45}$ spectra, and (b) Photoelectron $\mathrm{Ga} 2 \mathrm{p}_{3 / 2}$ spectrum from the reference GaN sample.

D. The Ga $2 p_{3 / 2}$ spectrum. The excess content of $\mathrm{Ga}$ in $\mathrm{GaCN}$ is also observed from the Ga $2 p_{3 / 2}$ photoelectron spectrum (Fig. 4.3 d). It is best deconvoluted by two GaussianLorentzian bands, instead of one. This suggests multiple bonding environment of Ga. The deconvolution reveals the $\mathrm{Ga}-\mathrm{Ga}$ band at $1116.5 \mathrm{eV}^{15}$ (occupying $66 \%$ band area), and a minor band at $1117.3 \mathrm{eV}$ (occupying the remaining area).

The suggested candidates for the minor band are $\mathrm{Ga}-\mathrm{N}$ and $\mathrm{Ga}-\mathrm{C}$ bonds. However, distinguishing between them is not straightforward due to the proximity of their $\mathrm{BE}$ values. For instance, in TMG fragments the $\mathrm{Ga}-\mathrm{C}$ bond is reported at $1117.2 \mathrm{eV}^{10}$. This energy is close to the $\mathrm{Ga}-\mathrm{N} \mathrm{BE}$ at $1117.8 \mathrm{eV}^{13}$. Limited by the instrumental resolution $(0.4 \mathrm{eV})$, additional deconvolution was not performed and the minor band can therefore be the result of both $\mathrm{Ga}-\mathrm{N}$ and $\mathrm{Ga}-\mathrm{C}$ bonds.

The excess $\mathrm{Ga}$ in $\mathrm{GaCN}$ (i.e., bonded as $\mathrm{Ga}-\mathrm{Ga}$ and $\mathrm{Ga}-\mathrm{C}$ ) originates from both the TMG fragments and from the completely-dissociated TMG; the latter in the form of elemental Ga clusters. Although the preferential sputtering of $\mathrm{N}$ over $\mathrm{Ga}$ during depth profiling is a reported phenomenon ${ }^{13}$, this doesn't seem to be the cause here and the Garichness of $\mathrm{GaCN}$ is perhaps genuine. This is confirmed by the $\mathrm{Ga} 2 \mathrm{p}_{3 / 2}$ spectrum of the reference GaN sample (Fig. 4.4 b). In the reference sample, the band is primarily composed of $\mathrm{Ga}-\mathrm{N}$ at $1117.6 \mathrm{eV} \mathrm{BE}$ (occupying $95 \%$ band area). The slight amount of $\mathrm{Ga}-\mathrm{Ga}$ bond (occupying the rest) is ascribed to the preferential sputtering of the $\mathrm{N}$. 
4.2.2.3 XRD analysis: XRD was used to further elucidate the GaCN composition. Fig. 4.5 compares the $\theta-2 \theta$ XRD scan of a GaCN layer with the GIXRD scan of the reference GaN layer $^{\dagger}$. Both materials exhibit a close match with hexagonal (wurtzitic) GaN crystals (compared with the database COD No. 96-901-1659).

The $d$-spacing for the first three peaks of the $\mathrm{GaCN}$ diffractogram (labelled (100), (002) and (101)) were estimated to be 2.77, 2.61 and $2.45 \AA$, respectively. From geometry, the $d$-spacing of wurtzitic GaN, with lattice constants $a=3.186 \AA$ and $c=5.186 \AA^{16}$, yield 2.76, 2.59 and $2.44 \AA$ for these planes, respectively. Moreover, the $d$-spacing obtained from the reference $\mathrm{GaN}$ sample were respectively 2.78, 2.63 and 2.50 $\AA$. From these observations, we conclude that the $\mathrm{GaCN}$ material consists of significant amount of wurtzitic $\mathrm{GaN}$ polycrystalline clusters.

Finally, all the diffraction peaks of the $\mathrm{GaCN}$ layer matched with $\mathrm{GaN}$ and no foreign peaks were observed. Therefore, the $\mathrm{GaCN}$ peaks are labelled with the same Miller indices as those of GaN. The lack of additional peaks, i.e., exclusive to the GaCN layer, points to the amorphous and/or nano-crystalline nature of the inclusions (besides the nanoscale GaN clusters) with grain sizes smaller than the detection limit of XRD.

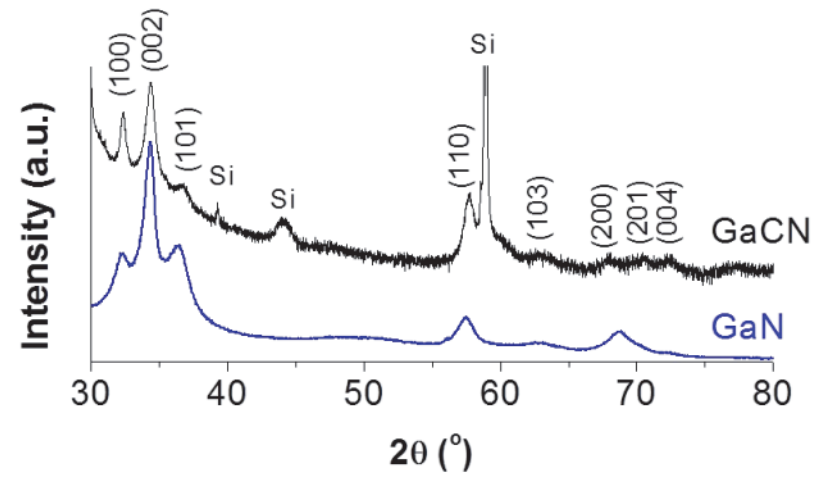

Fig. 4.5 $\theta-2 \theta$ XRD scan of a GaCN sample compared with GIXRD scan of the reference GaN sample.

\footnotetext{
$\dagger$ The different XRD techniques (i.e., $\theta-2 \theta$ and GIXRD) were used for the GaCN and GaN layers, respectively, due to the unavailability of the samples after a certain phase of research. However, the two techniques can equally detect the presence and position of the respective crystalline peaks, because the layers are polycrystalline. For the analysis, both layers were deposited on $\operatorname{Si}(111)$ substrates. The substrate does not produce a GIXRD pattern as its crystal planes are parallel to the surface. The $\theta-2 \theta$ scan detects such planes.
} 
4.2.2.4 HR-TEM and EF-TEM analyses: Fig. 4.6 a shows the TEM image of a $\mathrm{GaCN} / \mathrm{AlN} / \mathrm{Si}$ stack. Columnar structures in the $\mathrm{GaCN}$ can be observed at certain regions. The columns extend from the underlying AIN layer into the GaCN layer.

A. HR-TEM analysis. An HR-TEM image, zooming in the GaCN layer (Fig. 4.6 b), reveals its structural non-uniformity and an apparent phase-segregated morphology. The structure is categorized as containing the so-called (i) crystalline 'columns', (ii) spherical 'inclusions', and (iii) an interstitial 'matrix'.

The matrix reveals a range of $d$-spacing values obtained from Fast Fourier Transform (FFT) analysis at several regions. The co-existence of polycrystalline domains and amorphous clusters in the matrix is identified (Fig. $4.6 \mathrm{c}$ and insets). A quantified representation of the FFT analysis is shown in Fig. 4.7. Whereas matrix region 3 is almost amorphous, the peaks at matrix regions 1 and 2 (at 2.47, 2.58 and $2.78 \AA$ ) are in excellent agreement with the $d$-spacing of the (101), (002) and (100) wurtzitic GaN planes, respectively. Furthermore, FFT performed at the crystalline column of Fig. 4.6 b revealed a $d$-spacing of $2.74 \AA$, and therefore the column may be assigned to the (100) wurtzitic GaN plane. The peaks at the abnormally high $d$-spacing (5.70 and 6.10 $\AA$ ) (Fig. 4.7) are ascribed to Moiré fringes in the TEM micrographs.

In summary, the agreement between the estimated $d$-spacing from both TEM and XRD of $\mathrm{GaCN}$, and their match with the wurtzitic $\mathrm{GaN}$ reference, strongly suggests the abundance of $\mathrm{GaN}$ clusters in the $\mathrm{GaCN}$ layer.
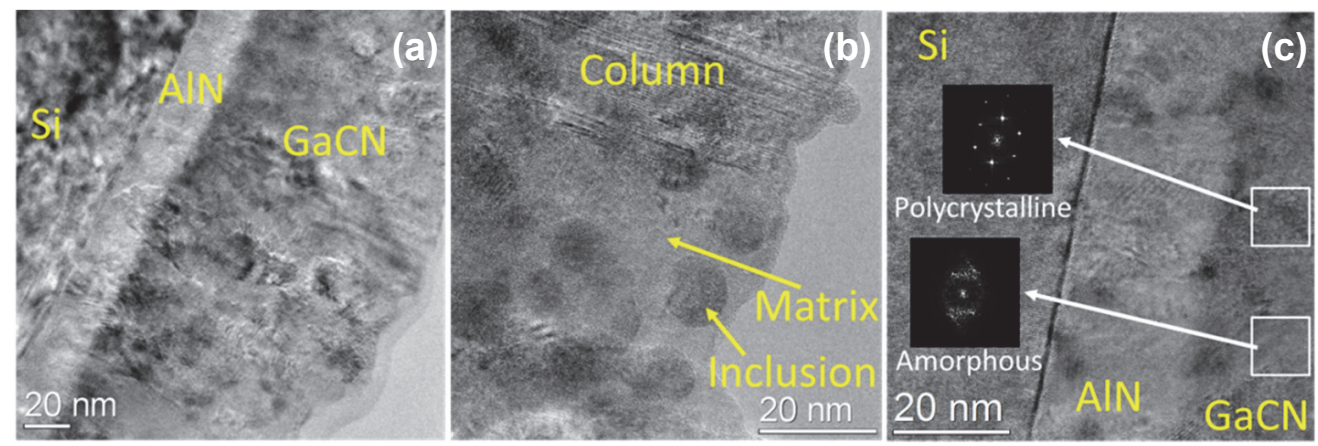

Fig. 4.6 HR-TEM images of (a) GaCN/AlN/Si stack, (b) the GaCN layer with its various structural features (Column, Matrix and Inclusion), (c) FFT images from poly- and amorphous regions of matrix.

\footnotetext{
$\$$ The quantified representation refers to the radial profile analysis of the FFT spectra of HR-TEM micrographs. The intensity of the spectra is plotted as a function of the radial distance, and the diffraction spots give rise to peaks in the intensity. The peak positions reveal the respective $d$-spacing. The radial profile analysis is an useful approach for a polycrystalline sample such as $\mathrm{GaCN}$. The analysis was performed by A. J. Onnink, University of Twente.
} 


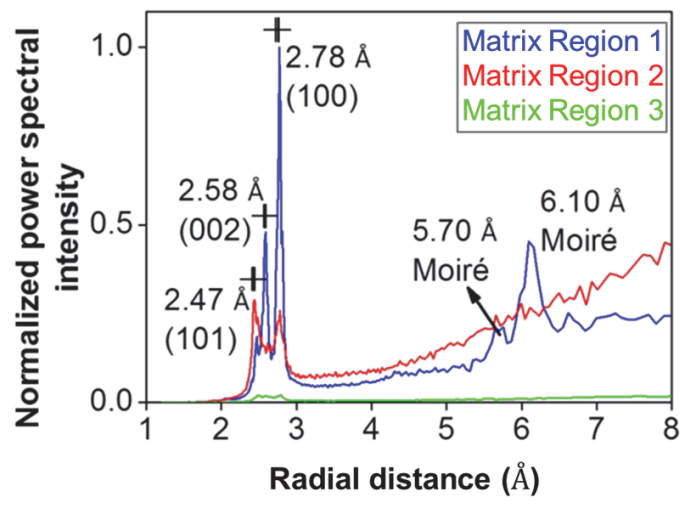

Fig. 4.7 Different $d$-spacing obtained from polycrystalline regions (1,2) and amorphous regions (3) of the matrix. (To note: The regions analysed in Fig. 4.7 may not correspond to Fig. $4.6 \mathrm{~b}$ and $\mathbf{c}$, as they are representative images only.)
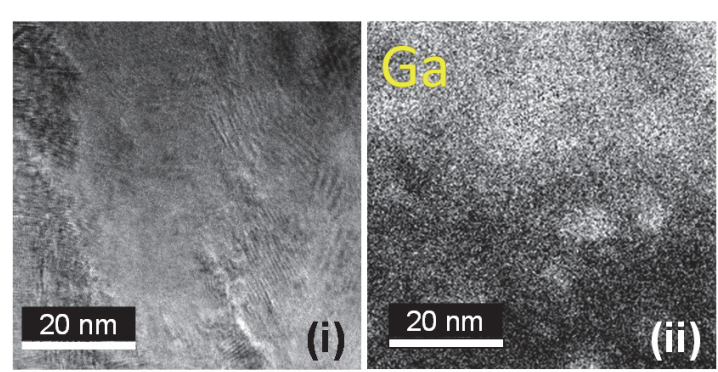

(a)

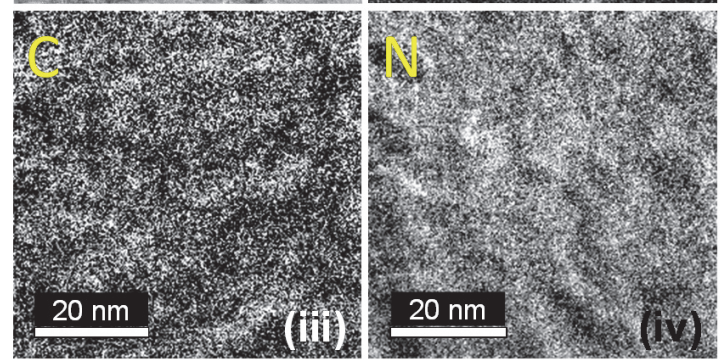

Fig. 4.8 (a) (i) Zero-energy-loss TEM image of $\mathrm{GaCN}$ where EF-TEM analysis was performed. (ii - iv) Elemental maps showing the spatial inhomogeneity of $\mathrm{Ga}, \mathrm{C}$ and $\mathrm{N}$, determined from 3window elemental mapping on their electron energy loss spectra (EELS). The brighter regions represent higher concentrations.

B. EF-TEM analysis. The phase-segregated nature of $\mathrm{GaCN}$ was revealed from EF-TEM analysis. The zero-loss TEM image, i.e., the region where the EF-TEM is performed, is shown in Fig. 4.8 a (i). As Fig. 4.8 a (ii-iv) reveals, the spatial distribution of the elements is hardly homogeneous, which otherwise could have indicated a single-phase GaCN layer. 
Specifically, Fig. 4.8 a (ii) shows Ga-rich inclusions - similar to that observed in Fig. 4.6 b. The sizes of these inclusions in both the figures are comparable: $8-11 \mathrm{~nm}$ in diameter, even though they are probed at different regions, and by different imaging modes (i.e., TEM and EF-TEM).

An overlay of the individual elemental maps of Fig. 4.8 a (ii-iv) is presented in Fig. 4.8 b. The caption to Fig. $4.8 \mathrm{~b}$ and the supporting Table 4.2 contains the detail of the analyses. Based on the spatial variation of the elemental intensities, we propose the existence of three separate chemical phases in $\mathrm{GaCN}$. They are Ga-, GaN- and CN-clusters.
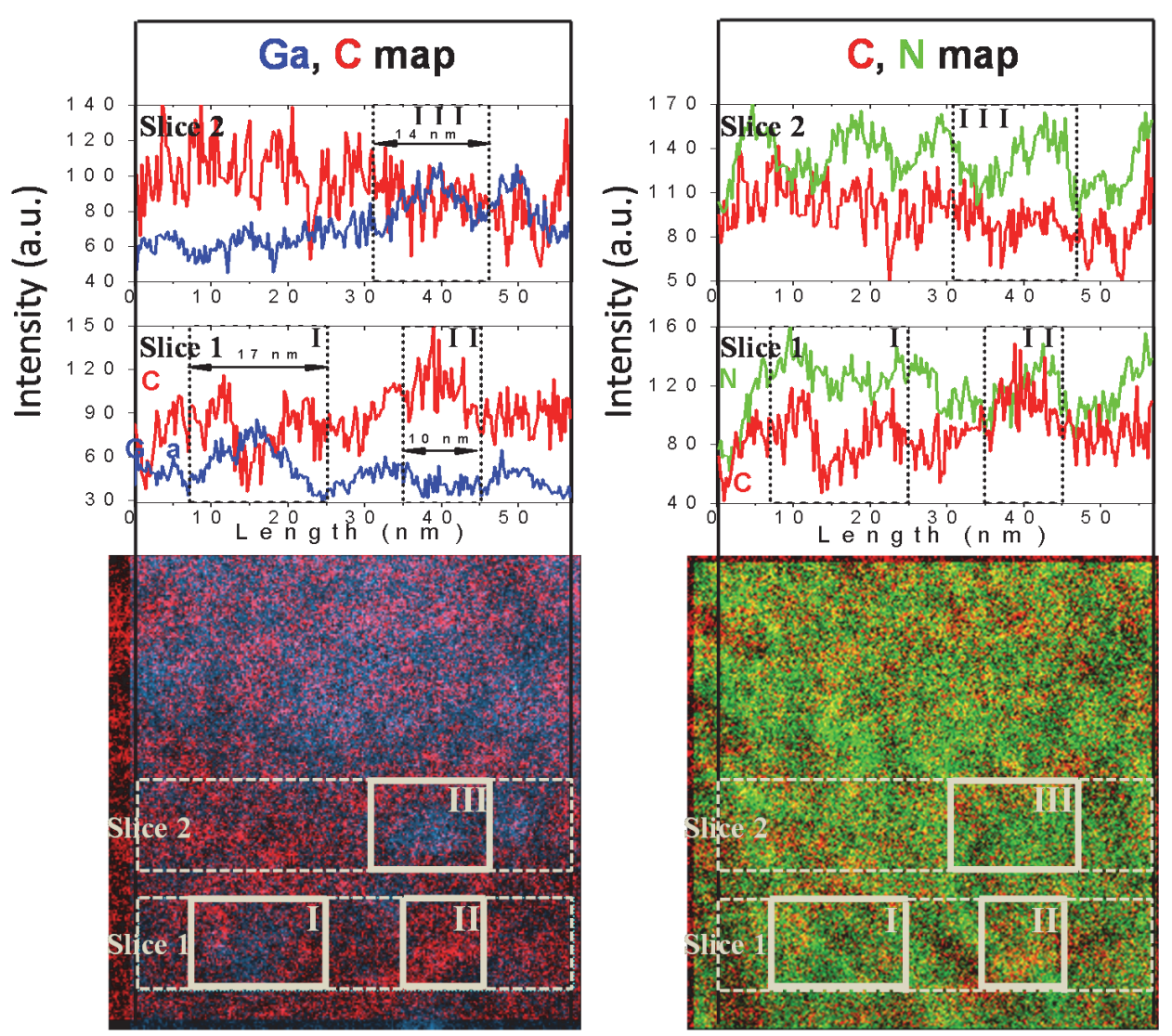

(b)

Fig. 4.8 (b) Lower images: Superimposed EF-TEM maps of Ga \& C and C \& N, corresponding to Fig. 4.8 a. Ga is in blue, $\mathrm{C}$ in red, and $\mathrm{N}$ in green. The specimen thickness is estimated to be $41 \mathrm{~nm}$. Two slices $(57 \times 12 \mathrm{~nm})$ and three regions-of-interest in these slices (regions I, II in slice 1; region III in slice 2) are selected for further analyses. Upper graphs: Spatial distribution of Ga \& C, and C \& N, in Slices 1and 2. Based on the correlation between the elemental intensities, the three regions have been identified to contain different chemical species. Summary of the analysis can be found in Table 4.2. 
Table 4.2 Assignment of various regions in the GaCN layer based on the EF-TEM maps of Fig. 4.8 b. Symbols $\uparrow, \downarrow$ and “-“ denote an increase, a decrease, and no significant change in the elemental intensities, respectively. Based on a simultaneous increase/decrease of the intensity of the elements, the regions have been assigned. For example, in region I, there is a finite but relatively-unchanging $\mathrm{N}$ intensity, whereas $\mathrm{Ga}$ and $\mathrm{C}$ intensities show simultaneous increase and decrease, respectively. The near-unchanging $\mathrm{N}$ intensity can be associated with $\mathrm{N}$-containing material above or below the region, and likely not associated with the region. Accordingly, region I is assigned to a Ga cluster.

\begin{tabular}{|c|c|c|c|c|c|}
\hline \multirow{2}{*}{ Region } & \multirow{2}{*}{ Size (nm) } & \multicolumn{3}{|c|}{ Intensity } & \multirow{2}{*}{ Assignment } \\
\cline { 3 - 5 } & & Ga & $\mathbf{C}$ & $\mathbf{N}$ & \\
\hline I & 17 & $\uparrow$ & $\downarrow$ & - & Ga cluster \\
\hline II & 10 & - & $\uparrow$ & $\uparrow$ & C=N cluster \\
\hline III & 14 & $\uparrow$ & - & $\uparrow$ & GaN cluster \\
\hline
\end{tabular}

\subsubsection{GaCN optical constants determined by SE}

The optical constants (i.e., refractive index $(n)$ and extinction coefficient $(k)$ ) of the reference GaN sample and $\mathrm{GaCN}$ layers with different compositions were determined by SE. The Kramers-Kronig consistent Sellmeier and B-spline-based optical models were used for this purpose ${ }^{\S}$. The variations of $n$ and $k$ of $\mathrm{GaN}$ and $\mathrm{GaCN}$ layers (with average compositions $\mathrm{Ga}_{0.66} \mathrm{C}_{0.03} \mathrm{~N}_{0.31}, \mathrm{Ga}_{0.70} \mathrm{C}_{0.06} \mathrm{~N}_{0.24}$ and $\mathrm{Ga}_{0.66} \mathrm{C}_{0.10} \mathrm{~N}_{0.24}$ ) with wavelength are shown in Fig. 4.9 a and $\mathbf{b}$, respectively. The $n$ of $\mathrm{GaN}$ in the dispersion-free range (i.e., 2.19 at $1500 \mathrm{~nm}$ ) is in agreement with that reported for poly-GaN films elsewhere (e.g., 2.16 at $1500 \mathrm{~nm})^{14}$.

Compared to $\mathrm{GaN}$, a consistently higher refractive index of the $\mathrm{GaCN}$ layers is revealed at all wavelengths (Fig. 4.9 a). From their compositions, it becomes apparent that carbon has a strong influence on the optical properties of $\mathrm{GaCN}$. Further, even a few atomic $\%$ increase of $\mathrm{C}$ results in a significant increase of the refractive index (Fig. 4.10 a, which shows the variation at $1500 \mathrm{~nm}$ ).

In the wavelength range studied, $\mathrm{GaCN}$ also has a higher $k$ compared to $\mathrm{GaN}$ (Fig. 4.9 b), again related directly to the carbon content. Fig. $4.10 \mathrm{~b}$ shows the variation of $k$ with $\mathrm{C}$-content at different wavelengths. From $n$ and $k$, the optical bandgaps $\left(E_{\mathrm{g}}\right)$ of $\mathrm{GaN}$ and the

$\S$ Details of the optical modelling procedure can be found in the supporting information to S. Banerjee et al., J. Phys. Chem. C, 2018, 122, 29567-29576. The optical modelling was performed by A. J. Onnink, University of Twente. 
$\mathrm{GaCN}$ layers were determined**. The C-content strongly influences the $E_{\mathrm{g}}$ of $\mathrm{GaCN}$ (Fig. $4.10 \mathrm{c}$ ).
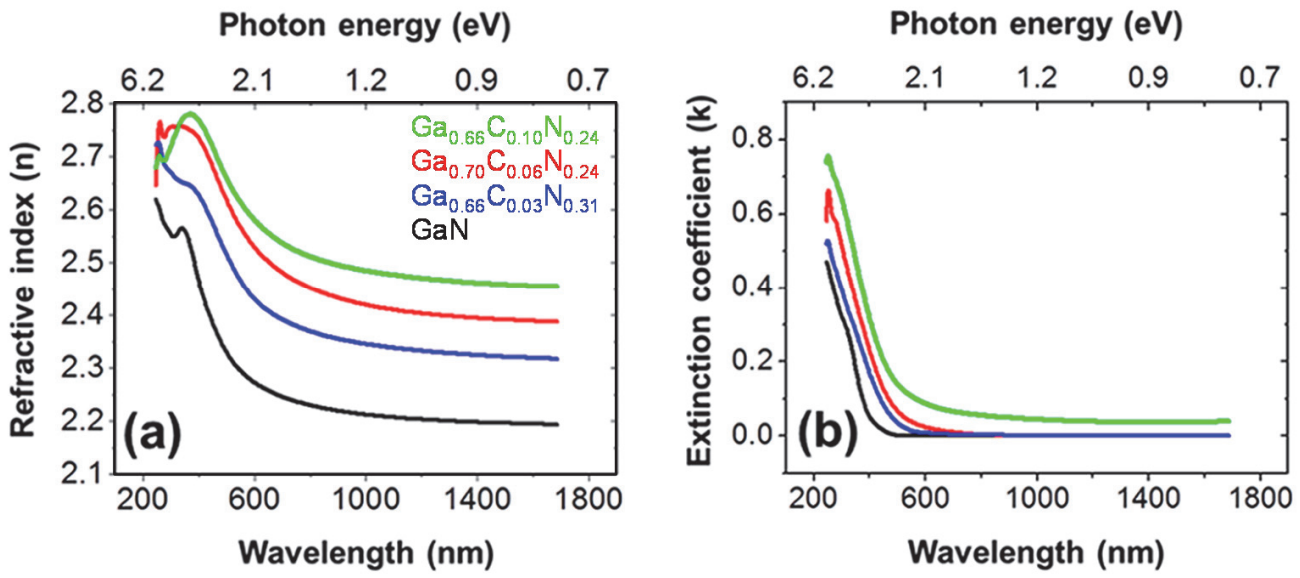

Fig. 4.9 Spectral variation of (a) refractive index and (b) extinction coefficient of $\mathrm{GaN}$ and $\mathrm{GaCN}$ layers with different $\mathrm{C}$ content. The average $\mathrm{GaCN}$ compositions are also indicated.
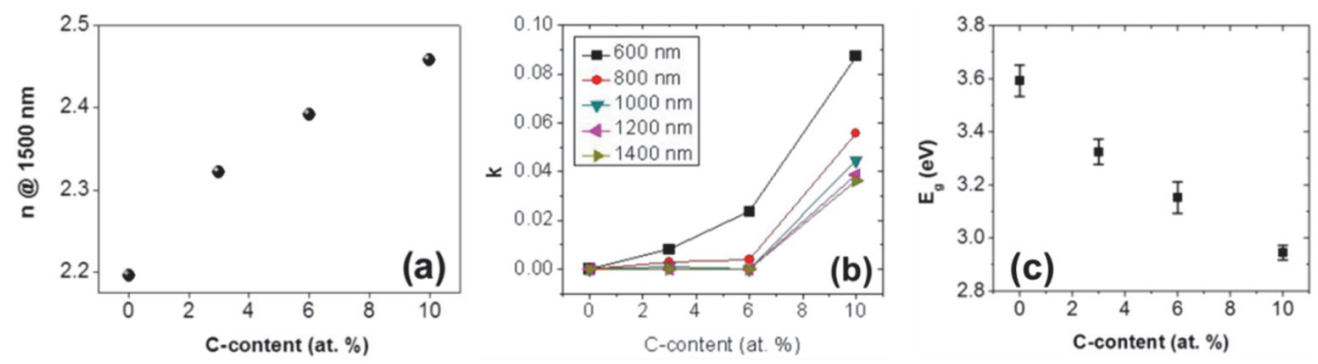

Fig. 4.10 (a) Increasing $n$, (b) $k$ and (c) decreasing $E_{\mathrm{g}}$ with C-content of $\mathrm{GaCN}$ layers. In (b), the variation of $k$ at sub-optical-bandgap wavelengths are shown.

\footnotetext{
** The $E_{\mathrm{g}}$ was determined from the so-called Tauc plots $^{39}$. Since $\mathrm{GaCN}$ is a composite material, $E_{\mathrm{g}}$ does not reflect the true optical bandgap of any single component, rather the onset of absorption in the composite. For constructing the Tauc plots, a power factor of 2 (i.e., corresponding to a direct bandgap semiconductor ${ }^{40}$ ) was used for both $\mathrm{GaN}$ and $\mathrm{GaCN}$ layers for fair comparison. The as-obtained $E_{\mathrm{g}}$ for $\mathrm{GaN}(3.59 \mathrm{eV})$ agrees with the reported poly-GaN $E_{\mathrm{g}}$ of $3.62 \mathrm{eV}^{41}$.
} 
4.2.3.1 Origin of the optical constants' variation: Because the literature on $\mathrm{GaCN}$ is hardly existent, we shall refer to other carbonitrides, such as $\mathrm{BCN}$ and $\mathrm{CN}$, to compare and explain the described optical properties of the GaCN layers.

A. In BCN and CN layers. Significant increase of refractive index and the decrease of optical bandgap with the film composition (i.e., the carbon and/or nitrogen contents) has been reported in $\mathrm{BCN}$ and $\mathrm{CN}$ layers ${ }^{8,17-26}$. The optical constants had been affected by mostly the C-content of these carbonitrides, in both single-phase and multiple-phase instances. In general, the index and the bandgap tend to increase with carbon. However, contradictory reports also exist. For example, Shi et al. reported a decrease of $E_{\mathrm{g}}$ with an increased relative share of the nitrogen-to-carbon contents in $\mathrm{CN}_{\mathrm{x}}$ layers ${ }^{26}$. Therefore, a more thorough literature study on the origin of the optical trends of the carbonitrides was done.

From the literature, it becomes evident that not just the overall $\mathrm{C}$-content, but specifically, the $\mathrm{sp}^{2} \mathrm{C}=\mathrm{N}$ and $\mathrm{C}=\mathrm{C}$ bonded clusters decrease the $E_{\mathrm{g}}$ (and increase the $n$ ) of carbonitrides. Moreover, relatively small amounts of the $\mathrm{sp}^{2}$ clusters have strong impact on the optical properties. For instance, Lei et al. ${ }^{17}$ showed that with an increase in the deposition temperature of $\mathrm{BCN}$ layers, the ratio of $\mathrm{sp}^{2} \mathrm{C}=\mathrm{N}$ clusters to $\mathrm{sp}^{3} \mathrm{~B}-\mathrm{N}$ and $\mathrm{C}-\mathrm{N}$ clusters in the layers decreased, which increased the $E_{\mathrm{g}}$ and reduced the $n$. Similarly, Kimura et al. ${ }^{18}$ reported a decrease of $E_{\mathrm{g}}$ (up to $0.9 \mathrm{eV}$ ) from only a minor (3 at. \%) increase in the $\mathrm{C}$-content. The authors attributed this to an increased share of amorphous regions, rich in $\mathrm{sp}^{2} \mathrm{C}=\mathrm{C}$ and $\mathrm{C}=\mathrm{N}$ clusters, in the $\mathrm{BCN}$ layers. The share of the $\mathrm{sp}^{3} \mathrm{~B}-\mathrm{N}$ and $\mathrm{C}-\mathrm{N}$ clusters, on the contrary, decreased with the increasing carbon content. The same authors in another publication demonstrated two BCN samples having an equal C-content $\left(\sim 13\right.$ at. \%), but with an $E_{\mathrm{g}}$ difference of $\sim 1 \mathrm{eV}^{25}$. The lower- $E_{\mathrm{g}}$ sample revealed a higher amount of $\mathrm{sp}^{2}$ - over $\mathrm{sp}^{3}$-bonded $\mathrm{CN}$ clusters.

If indeed the share of carbonaceous amorphous clusters containing the $\mathrm{sp}^{2}$ bonds increases with the $\mathrm{C}$-content, then this coincides with similar observations made by other researchers on the role of such amorphous clusters on the optical properties of BCN layers ${ }^{20 \text {, }}$ 27. Moreover, not only in $\mathrm{BCN}$, but also in the afore-mentioned $\mathrm{CN}_{\mathrm{x}}$ example ${ }^{26}$, the reason behind the decrease of $E_{\mathrm{g}}$ has been attributed to a higher fraction of $\mathrm{sp}^{2}$ - over $\mathrm{sp}^{3}$ - bonded amorphous carbonaceous clusters. 

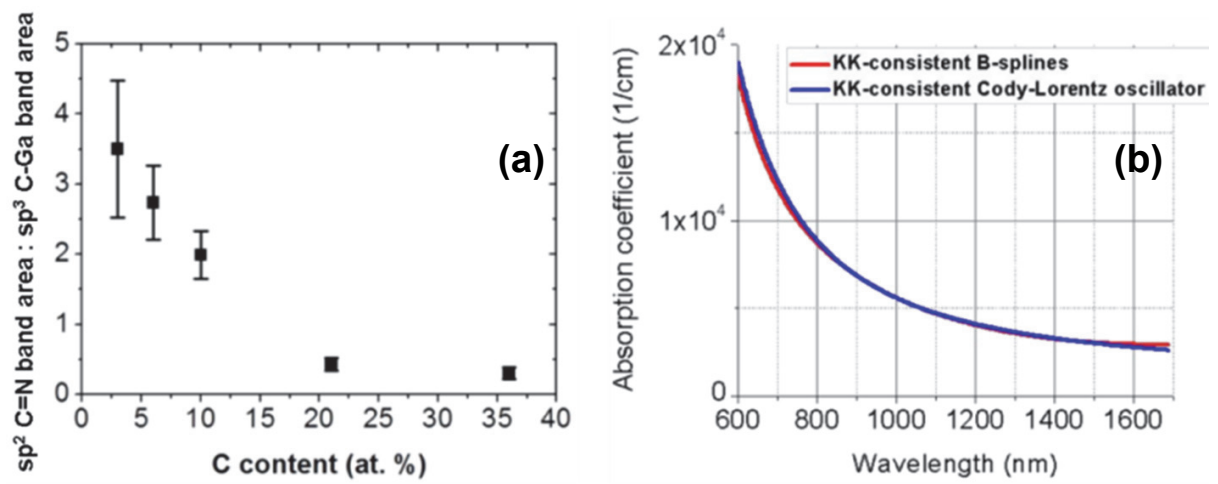

Fig. 4.11 (a) Variation of the ratio of the $\mathrm{sp}^{2} \mathrm{C}=\mathrm{N}$ and $\mathrm{sp}^{3} \mathrm{C}-\mathrm{Ga}$ band areas with $\mathrm{C}$-content in $\mathrm{GaCN}$. (The former band has its XPS signature at $286.3 \mathrm{eV}$ and the latter band has its signature at $283.5 \mathrm{eV}$.) (b) Spectral variation of absorption coefficient $(\alpha)$ of $\mathrm{Ga}_{0.66} \mathrm{C}_{0.10} \mathrm{~N}_{0.24}$ in the sub-bandgap wavelength range. The plots are obtained using Kramers-Kronig consistent B-spline (in red) and Cody-Lorentz (in blue) parameterization of the optical functions.

B. In GaCN layers. The XPS of the GaCN layers demonstrates, rather anomalous to their optical trends, a decreasing $\mathrm{sp}^{2}$-to-sp ${ }^{3}$ ratio with an increasing C-content (Fig. 4.11 a). For example, in the $\mathrm{Ga}_{0.66} \mathrm{C}_{0.03} \mathrm{~N}_{0.31}$ sample the ratio of the $286.3 \mathrm{eV}$ band (representing sp ${ }^{2} \mathrm{C}=\mathrm{N}$ ) to the $283.5 \mathrm{eV}$ band (representing sp ${ }^{3} \mathrm{C}-\mathrm{Ga}$ ) is the largest. This shows, when the overall $\mathrm{C}$ content is low (3 at. \%), it mainly consists of $\mathrm{sp}^{2} \mathrm{C}=\mathrm{N}$ clusters. This explains the changes in the $\mathrm{GaCN}$ optical constants already at the low C-levels.

The share of the $\mathrm{sp}^{2}$-bonded $\mathrm{C}=\mathrm{N}$ clusters, relative to the $\mathrm{sp}^{3}$-bonded $\mathrm{C}$-Ga clusters, reduces as the $\mathrm{C}$-content increases: beyond 10 at. $\% \mathrm{C}$ almost the entire carbon is in the $\mathrm{sp}^{3}$ form. The $E_{\mathrm{g}}$ shows a steady decrease up to 10 at. $\% \mathrm{C}$ (Fig. $4.10 \mathrm{c}$ ). It is speculated that beyond the 10 at. \% carbon (e.g., at 20 and 35 at. \%, Fig. 4.11 a), the trend in $E_{\mathrm{g}}$ could change. Unfortunately, the C-rich layers were too thin (sub-10 $\mathrm{nm}$ ) to accurately determine their optical constants by SE. On the basis of the above discussion on $\mathrm{BCN}$ and $\mathrm{CN}$ layers, we tentatively assign the origin of the low $E_{\mathrm{g}}$ (and high $n$ ) of $\mathrm{GaCN}$ to the presence of the $\mathrm{sp}^{2}$ $\mathrm{C}=\mathrm{N}$ clusters.

C. On the high ' $\boldsymbol{k}$ ' of $\mathbf{G a C N}$. Strong sub-optical-bandgap absorption is observed in all the $\mathrm{GaCN}$ layers that increases with the C-content (Fig. 4.10 b). The possible cause is attributed to electronic transitions in (i) the matrix and the inclusions surrounding the GaN crystals (recall Fig. 4.6 b), and/or (ii) the GaN crystals themselves involving states that arise from defects in the lattice ${ }^{28}$, possibly due to carbon at the interfaces of these crystals. The second 
case implies that the absorption coefficient $(\alpha)$ (derived from $k$ as $4 \pi k / \lambda$ ) below the optical bandgap follows the Urbach relation: $\alpha \propto \exp \left(E / E_{\mathrm{u}}\right)$ (where $E_{\mathrm{u}}$ is the Urbach energy ${ }^{29}$ ). The $\mathrm{Ga}_{0.66} \mathrm{C}_{0.10} \mathrm{~N}_{0.24}$ sample was chosen as the test-case to analyse the nature of the subbandgap absorption. The $\alpha$ values obtained from the B-spline optical model (Fig. 4.11 b, red) are rather consistent with the Urbach relation ${ }^{\dagger \dagger}$. This suggest to ascribe the sub-bandgap absorption in the $\mathrm{GaCN}$ layers to the carbon-induced defect states in the GaN poly-crystals. However, the first case may not also be completely excluded since the inclusions (or the matrix) may contain species that produce spectral absorption of a similar shape. An alternate possibility of the presence of a single-phase $\mathrm{GaCN}$ material is ignored since it was not detected by the prior analyses.

\subsection{GaCN growth kinetics}

From the previous section, it is evident that the ability to tune the C-content of $\mathrm{GaCN}$ layers enables a wide range of optical constants. An accurate tuning can be achieved if the growth mechanism of the composite is known. However, since this composite is hitherto unreported, a possible mechanism of the composite formation is proposed in the rest of the chapter.

The material characterization (Section 4.2.2) concluded the co-existence of a number of phases in the $\mathrm{GaCN}$ composite: $\mathrm{GaN}, \mathrm{Ga}$ and $\mathrm{C}=\mathrm{N}$ clusters, bonded in several chemical fashions. We propose that the formation of such a composite occurs due to an interplay between two chemical pathways - one contributing to the formation of the polycrystalline GaN clusters, and the other contributing to the formation of the inclusions. These two pathways are subsequently addressed.

\subsubsection{Formation of GaN clusters}

In this project, a novel chemical pathway has been conceived (speculated) of the surface reactions between $\mathrm{TMG}$ and $\mathrm{NH}_{3}$, that can be activated beyond $350{ }^{\circ} \mathrm{C}$ (reference ${ }^{30}$ and Chapter 5). In the $\mathrm{GaCN}$ layers, the formation of the $\mathrm{GaN}$ poly-crystals is speculated to occur by this chemical route. Only a limited description of the pathway will be presented in this chapter, because the complete description is provided in the next chapter (Section 5.3).

\footnotetext{
† This is identified because the B-spline result is consistent with the Cody-Lorentz optical model, which has an in-built feature for modelling the Urbach tail (Fig. 4.11 b, blue). The graph was contributed by A. J. Onnink, University of Twente.
} 
The pathway relies on the strong adduct-forming tendency of the Lewis acid TMG and the Lewis base $\mathrm{NH}_{3}{ }^{31}$, not only in the gas-phase as occurs in the metalorganic chemical vapor deposition (MOCVD) of GaN (Reaction 4.1 $)^{32}$, but presumably also on the surface ${ }^{30}$. The surface adduct formation is speculated to play an important role in the pulsed-precursorinduced $\mathrm{GaCN}$ growth. The speculation of the existence of the surface adduct is supported by the reported existence of a similar surface adduct between TMA and $\mathrm{NH}_{3}$, observed experimentally ${ }^{33}$.

$$
\left(\mathrm{CH}_{3}\right)_{3}-\mathrm{Ga}+\mathrm{NH}_{3} \rightleftharpoons\left(\mathrm{CH}_{3}\right)_{3}-\mathrm{Ga}: \mathrm{NH}_{3} \quad \text { Reaction } 4.1
$$

In the gas-phase reactions between $\mathrm{TMG}$ and $\mathrm{NH}_{3}$, the adduct formation is reported to be reversible in nature ${ }^{32}$, due to the modest difference in the electronegativity values of $\mathrm{Ga}$ (1.81) and $\mathrm{C}(2.55)^{34}$. Therefore, its rate is strongly dependent on the $\mathrm{NH}_{3}$ partial pressure (or $\left.P_{\mathrm{NH} 3}\right)^{35}$. The surface adduct formation can also be presumed to be reversible like its gasphase counterpart, because the same effects of electronegativity are expected to apply on the surface. Therefore, the adduct formation is presumably dependent on $P_{\mathrm{NH} 3}$. The surface adduct forms on the interaction of $\mathrm{NH}_{3}$ to a TMG-chemisorbed surface site. The reaction can be expressed as:

$\left(\mathrm{CH}_{3}\right)_{\mathrm{x}}-\mathrm{Ga}_{(\mathrm{s})}+\mathrm{NH}_{3} \rightleftharpoons\left(\mathrm{CH}_{3}\right)_{\mathrm{x}}-\mathrm{Ga}: \mathrm{NH}_{3(\mathrm{~s})} \quad$ Reaction 4.2

\{where $\left(\mathrm{CH}_{3}\right)_{\mathrm{x}}-\mathrm{Ga}_{(\mathrm{s})}$ represents TMG in the chemisorbed form, $\mathrm{x}=1-2$;

$\left(\mathrm{CH}_{3}\right)_{\mathrm{x}}-\mathrm{Ga}: \mathrm{NH}_{3(\mathrm{~s})}$ represents the surface adduct\}

The overall GPC of $\mathrm{GaCN}$ is assumed to be determined to a large extent by the formation of the GaN poly-crystals alone, and not by the other inclusions (this assumption will be verified in Section 4.3.2). The GPC indeed shows a dependence on the $P_{\mathrm{NH} 3}$ (Fig. 4.12 a, blue). The so-observed pressure dependence is atypical for an ALD process, but can be explained in terms of the surface-adduct pathway and its dependence on $P_{\mathrm{NH} 3}$ (Reaction 4.2). The GaCN GPC is compared with that of the reference ALD GaN sample for various $P_{\mathrm{NH} 3}$ values (Fig. 12 a, black). The $P_{\mathrm{NH} 3}$-dependence in both cases make us (for now) speculate that the $\mathrm{GaN}$ poly-crystals in the $\mathrm{GaCN}$ composite are formed through the same surface adduct route as in the reference GaN sample.

\subsubsection{Formation of Ga- and C-containing inclusions}

In addition to forming the surface adduct with $\mathrm{NH}_{3}$, the chemisorbed-TMG also has the possibility to dissociate into 'fragments' at the GaCN growth temperatures, which range from 450 to $600{ }^{\circ} \mathrm{C}$. TMG being an metalorganic, is hardly stable above $400{ }^{\circ} \mathrm{C}$ in the gasphase $^{36}$, and above $150{ }^{\circ} \mathrm{C}$ on the surface ${ }^{37}$. At the growth temperatures of the layers, the dissociation would result in the deposition of $\mathrm{DMG}, \mathrm{MMG}$, free $\mathrm{Ga}$, hydrocarbons and 
carbon on the surface ${ }^{10}$. Such species are likely unable to form the GaN poly-crystals through the surface adduct route. Instead, continued deposition would lead to their accumulation as the inclusions earlier shown in Fig. 4.6 b.

Due to the presumed strong $P_{\mathrm{NH} 3}$ dependency of the GPC of the GaN poly-crystals, their formation reduces rapidly at low $P_{\mathrm{NH} 3}$ values (Fig. 4.12 a, black and blue). Still, the formation of the inclusions continue because of the ongoing TMG dissociation, that does not necessarily has the same pressure dependence.

To investigate the above, keeping the temperature the same as in Fig. 4.12 a (550 ${ }^{\circ} \mathrm{C}$ ), a GaCN deposition was performed at the very low $P_{\mathrm{NH} 3}$ of $0.3 \times 10^{-3}$ mbar. This resulted in an extremely low GPC, with a mere $0.5 \mathrm{~nm}$ film formation after 900 ALD cycles. Instead, the substrate (AIN) was covered with a multitude of unusually-shaped particles (Fig. 4.12 b). The particles typically consisted of a spherical lobe with several protruding 'ears' (see inset, which shows the ESB image of one). From the ESB image (and supported by an EDX analysis; not shown), it was identified that the sphere was Ga-rich and the ears were C-rich. Nitrogen was not detected in the particles. Formation of such nanoparticles had been reported elsewhere, where their origin was attributed to the dissociation of $\mathrm{TMG}^{38}$. Similar is speculated to occur in our case.

The competing mechanisms of GaN polycrystal formation and TMG dissociation during $\mathrm{GaCN}$ growth are evident from the range of GPC values obtained at various deposition temperatures and $P_{\mathrm{NH} 3}$ (Fig. 4.13 a). At both pressures (i.e., at the high $P_{\mathrm{NH} 3}$ of 7.25 mbar and at the low $P_{\mathrm{NH} 3}$ of $0.7 \times 10^{-3} \mathrm{mbar}$ ), increasing the temperature initially increases the GPC due to an enhancement of the polycrystal formation. However, at higher temperatures (i.e., beyond 450 or $500{ }^{\circ} \mathrm{C}$ ), the GPC increase eventually tends to slow down (or even becomes negative) as the TMG dissociation rate surpasses the $\mathrm{GaN}$ formation rate. Raising the temperature eventually depletes the TMG from forming the GaN poly-crystals through the surface adduct route, thereby reducing the overall GPC of the GaCN composite.

Fig. 4.13 b summarizes the role of temperature and $P_{\mathrm{NH} 3}$ on the C-content of $\mathrm{GaCN}$. At every temperature studied, decreasing the $P_{\mathrm{NH} 3}$ reduces the GPC and increases the Ccontent due to the suppression of the GaN formation, but not the TMG dissociation. This is also shown in the inset to Fig. $\mathbf{4 . 1 3}$ a, for the two pressures studied. Similarly, at every $P_{\mathrm{NH} 3}$, raising the temperature increases the $\mathrm{C}$-content by an enhancement of TMG dissociation. Fig. 4.13 $\mathrm{b}$ thus serves as a reference for preparing $\mathrm{GaCN}$ layers with the desired C-content and therefore having the desired optical properties. 

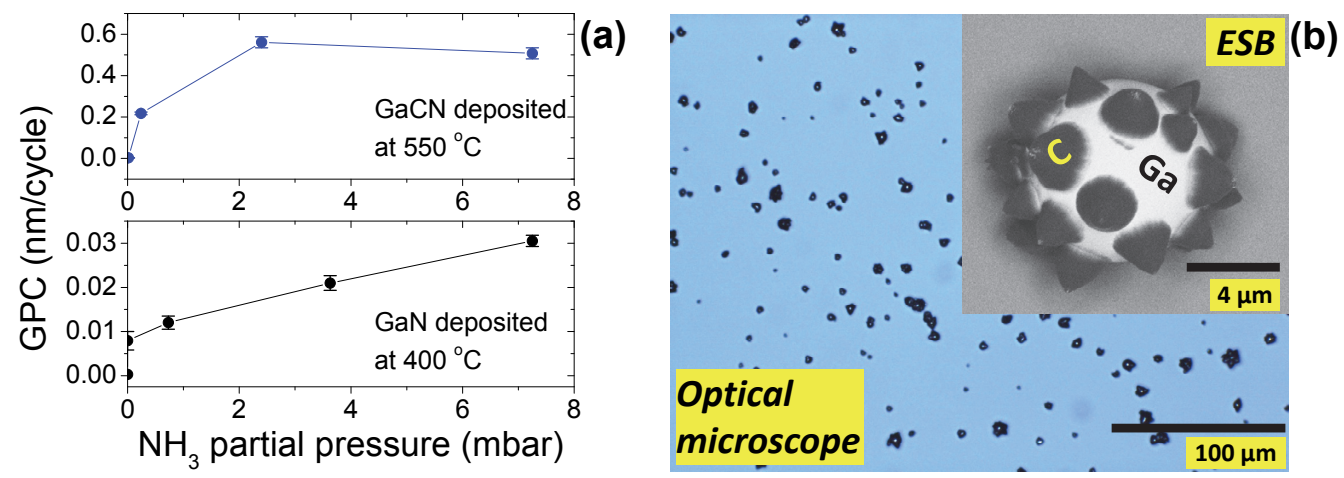

Fig. 4.12 (a) Variation of GPC with $P_{\mathrm{NH} 3}$ of $\mathrm{GaCN}$ and GaN layers. (b) Optical microscope image: particles, with a mean size of $5 \mu \mathrm{m}$, deposited at a low $P_{\mathrm{NH} 3}$ of $0.3 \times 10^{-3}$ mbar. ESB image: Zoom-in to a single particle, revealing a Ga-rich sphere and C-rich ears.
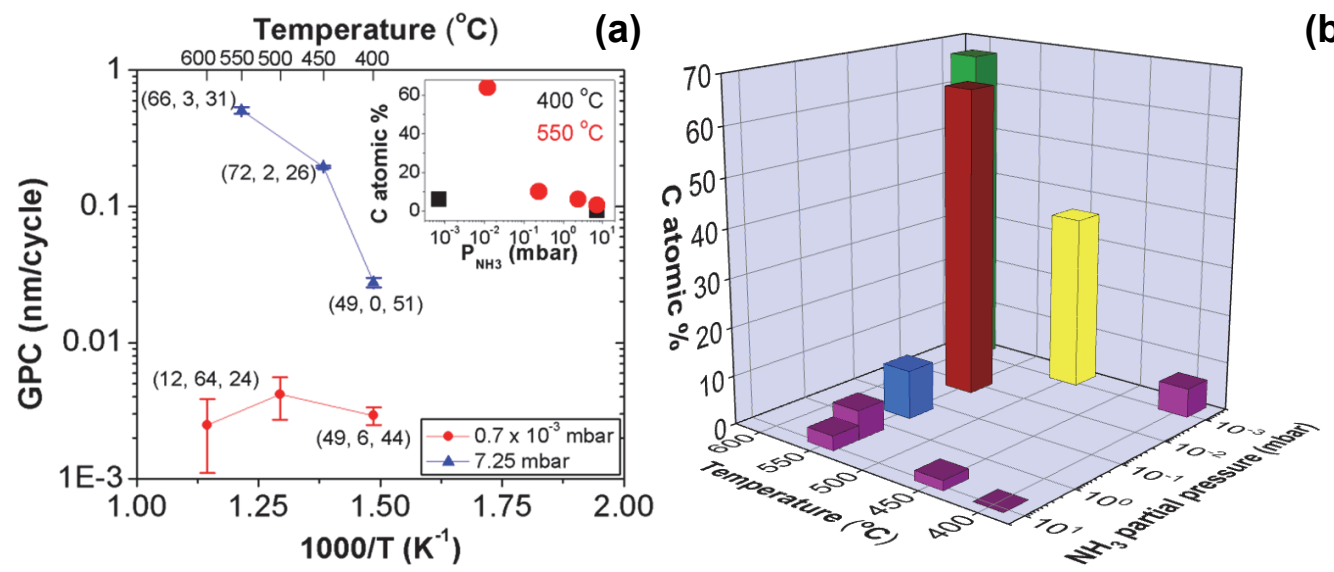

(b)

Fig. 4.13 (a) Variation of GPC of GaCN layers of a wide composition range, deposited under different temperatures and $P_{\mathrm{NH} 3}$. The XPS film composition is indicated next to the data-points (e.g., $(49,6,44)$ corresponds to $\left.\mathrm{Ga}_{0.49} \mathrm{C}_{0.06} \mathrm{~N}_{0.44}\right)$. Inset: C-content of $\mathrm{GaCN}$ deposited at 400 and $550{ }^{\circ} \mathrm{C}$.

(b) Variation of C-content of $\mathrm{GaCN}$ with temperature and $P_{\mathrm{NH} 3}$. 


\subsection{Conclusions}

This chapter introduced novel composite $\mathrm{GaCN}$ layers prepared by alternate pulses of $\mathrm{TMG}$ and $\mathrm{NH}_{3}$ precursors at various temperatures and $\mathrm{NH}_{3}$ partial pressures. Using a wide array of characterization techniques (FTIR, XPS, XRD and TEM), it was concluded that $\mathrm{GaCN}$ was not a homogenous single-phase material, but rather a composite of polycrystalline wurtzitic $\mathrm{GaN}$, and Ga-, $\mathrm{C}-$, and $\mathrm{N}$-containing inclusions, phase-segregated at the nanoscale and bonded in various chemical fashions.

The C-content had a profound effect on the optical properties of the composite. A decrease in $E_{\mathrm{g}}$ from $3.59 \mathrm{eV}$ in $\mathrm{GaN}$ to $2.95 \mathrm{eV}$ in $\mathrm{GaCN}$ was achieved with 10 at. \% of carbon. The refractive index accordingly increased from 2.20 to 2.46 at $1500 \mathrm{~nm}$. From the literature on other carbonitrides like $\mathrm{BCN}$ and $\mathrm{CN}$, it was identified that not just the overall $\mathrm{C}$-content, but specifically the ratio of the $\mathrm{sp}^{2}$-to-sp ${ }^{3}$ hybridized carbonaceous clusters determine the optical properties. In $\mathrm{GaCN}$, the $\mathrm{sp}^{2}-\mathrm{C}=\mathrm{N}$ clusters located in the interstitial matrix was proposed to cause the low optical bandgap and the high refractive index. The strong absorption at the sub-optical-bandgap wavelengths was due to carbon residing at the interface of the $\mathrm{GaN}$ poly-crystals.

In order to tune the $\mathrm{C}$-content of $\mathrm{GaCN}$, the growth mechanism was investigated, and a novel chemical pathway involving the surface adduct formation between TMG and $\mathrm{NH}_{3}$ was proposed. This consisted of studying the influence of the deposition temperature and the $\mathrm{NH}_{3}$ partial pressure on the GPC and the composition of $\mathrm{GaCN}$ layers. Two separate chemical pathways presumably compete during the growth: (i) the surface adduct pathway, which results in the GaN poly-crystals, and (ii) the dissociation of TMG, which forms the Ga- and C-containing inclusions. As a final note, a potential optical device utilizing the $\mathrm{GaCN}$ layers is conceptualized in Appendix 4. 


\section{References}

1. C. Hwang, M. Schurman, W. Mayo, Y. Lu, R. Stall and T. Salagaj, Journal of Electronic Materials, 1997, 26, 243-251.

2. B. Hájek, V. Kohout and V. Flemr, Monatshefte für Chemie-Chemical Monthly, 1986, 117, 1157-1164.

3. T. Miyazaki, K. Takada, S. Adachi and K. Ohtsuka, Journal of Applied Physics, 2005, 97, 093516.

4. G. Socrates, Infrared and Raman Characteristic Group Frequencies: Tables and Charts, John Wiley \& Sons, 2004.

5. B. Deb, A. Ganguly, S. Chaudhuri, B. Chakraborti and A. Pal, Materials Chemistry and Physics, 2002, 74, 282-288.

6. J. Durig and K. Chatterjee, Journal of Raman Spectroscopy, 1981, 11, 168-173.

7. T. L. Barr and S. Seal, Journal of Vacuum Science \& Technology A: Vacuum, Surfaces, and Films, 1995, 13, 1239-1246.

8. A. Prakash and K. B. Sundaram, Applied Surface Science, 2017, 396, 484-491.

9. J. Sawahata, M. Teramoto, S. Nakamura, K. Kametomo, M. Satake, S.-i. Yamamoto, K. Itoh and K. Takarabe, Japanese Journal of Applied Physics, 2014, 53, 071002.

10. F. Lee, A. Backman, R. Lin, T. Gow and R. Masel, Surface Science, 1989, 216, 173-188.

11. B. Maa and P. Dapkus, Journal of Electronic Materials, 1990, 19, 289-294.

12. M. Alevli, A. Haider, S. Kizir, S. A. Leghari and N. Biyikli, Journal of Vacuum Science \& Technology A: Vacuum, Surfaces, and Films, 2016, 34, 01A137.

13. R. Carin, J. Deville and J. Werckmann, Surface and Interface Analysis, 1990, 16, 65-69.

14. C. Ozgit-Akgun, E. Goldenberg, A. K. Okyay and N. Biyikli, Journal of Materials Chemistry C, 2014, 2, 2123-2136.

15. G. Schön, Journal of Electron Spectroscopy and Related Phenomena, 1973, 2, 75-86.

16. M. E. Levinshtein, S. L. Rumyantsev and M. S. Shur, Properties of Advanced Semiconductor Materials: GaN, AIN, InN, BN, SiC, SiGe, John Wiley \& Sons, 2001.

17. M. Lei, Q. Li, Z. Zhou, I. Bello, C. Lee and S. Lee, Thin Solid Films, 2001, 389, 194-199.

18. C. Kimura, H. Sota, H. Aoki and T. Sugino, Diamond and Related Materials, 2009, 18, 478481.

19. V. O. Todi and K. B. Sundaram, Electrochemical and Solid-State Letters, 2011, 14, G49G51. 
20. V. S. Sulyaeva, Y. M. Rumyantsev, V. G. Kesler and M. L. Kosinova, Thin Solid Films, 2015, 581, 59-64.

21. A. Weber, U. Bringmann, R. Nikulski and C.-P. Klages, Diamond and Related Materials, 1993, 2, 201-206.

22. T. Sugino and H. Hieda, Diamond and Related Materials, 2000, 9, 1233-1237.

23. R. Levy, E. Mastromatteo, J. Grow, V. Paturi, W. Kuo, H. Boeglin and R. Shalvoy, Journal of Materials Research, 1995, 10, 320-327.

24. H. Ahn, K. Klimek and K.-T. Rie, Surface and Coatings Technology, 2003, 174, 1225-1228.

25. H. Sota, C. Kimura, H. Aoki and T. Sugino, Diamond and Related Materials, 2010, 19, 1441-1445.

26. J. Shi, Y. Xu and J. Zhang, Thin Solid Films, 2005, 483, 169-174.

27. T. Yuki, S. Umeda and T. Sugino, Diamond and Related Materials, 2004, 13, 1130-1134.

28. S. John, C. Soukoulis, M. H. Cohen and E. Economou, Physical Review Letters, 1986, 57, 1777 .

29. O. Stenzel, The Physics of Thin Film Optical Spectra, Springer, 2005.

30. S. Banerjee and A. Y. Kovalgin, ECS Transactions, 2018, 86, 21-29.

31. M. J. Almond, C. E. Jenkins, D. A. Rice and K. Hagen, Journal of Organometallic Chemistry, 1992, 439, 251-261.

32. J. R. Creighton and G. T. Wang, The Journal of Physical Chemistry A, 2005, 109, 133-137.

33. M. Bartram, T. Michalske, J. Rogers Jr and R. Paine, Chemistry of Materials, 1993, 5, 14241430.

34. A. Allred, Journal of Inorganic and Nuclear Chemistry, 1961, 17, 215-221.

35. J. R. Creighton and G. T. Wang, The Journal of Physical Chemistry A, 2005, 109, 1055410562.

36. C. Larsen, N. Buchan, S. Li and G. Stringfellow, Journal of Crystal Growth, 1990, 102, 103116.

37. M. J. Bronikowski and R. J. Hamers, Surface Science, 1996, 348, 311-324.

38. J. R. Creighton, G. T. Wang and M. E. Coltrin, Journal of Crystal Growth, 2007, 298, 2-7.

39. J. Tauc, R. Grigorovici and A. Vancu, Physica Status Solidi (b), 1966, 15, 627-637. 
40. T. S. Moss, G. J. Burrell and B. Ellis, Semiconductor Opto-Electronics, ButterworthHeinemann, 2013.

41. P. Motamedi, N. Dalili and K. Cadien, Journal of Materials Chemistry C, 2015, 3, 74287436.

42. C. Sheppard, Pure and Applied Optics: Journal of the European Optical Society Part A, $1995,4,665$.

43.. $\quad$ P. Yeh, Optical Waves in Layered Media, Wiley-Interscience, 2005.

44. J. Dorsaz, J.-F. Carlin, S. Gradecak and M. Ilegems, Journal of Applied Physics, 2005, 97, 084505 .

45. D.-X. Wang, I. T. Ferguson and J. A. Buck, Applied Optics, 2007, 46, 4763-4767.

46. K. Kishino, M. S. Unlu, J.-I. Chyi, J. Reed, L. Arsenault and H. Morkoc, IEEE Journal of Quantum Electronics, 1991, 27, 2025-2034.

47. E. Ozbay, M. S. Islam, B. Onat, M. Gokkavas, O. Aytur, G. Tuttle, E. Towe, R. Henderson and M. S. Unlu, IEEE Photonics Technology Letters, 1997, 9, 672-674.

48. T.-C. Lu, C.-C. Kao, H.-C. Kuo, G.-S. Huang and S.-C. Wang, Applied Physics Letters, 2008, 92, 141102.

49. H. Yao, C. Lin, H. Kuo and S. Wang, Journal of Crystal Growth, 2004, 262, 151-156.

50. T. Someya and Y. Arakawa, Applied Physics Letters, 1998, 73, 3653-3655.

51. H. Ng, T. Moustakas and S. Chu, Applied Physics Letters, 2000, 76, 2818-2820.

52. D. I. Babic and S. W. Corzine, IEEE Journal of Quantum Electronics, 1992, 28, 514-524. 


\section{Appendix 4: Exploiting the high refractive index of GaCN in Distributed Bragg Reflector (DBR)}

A 4.1 Principle of DBR: A DBR is a passive optical device that reflects light only at a certain design-wavelength and filters out the other wavelengths. It consists of a stack of alternating high and low refractive indices. The thicknesses of each pair are so designed that light of only desired or the 'design' wavelength constructively interferes and gets reflected by the stack. The other wavelengths interfere destructively and are attenuated.

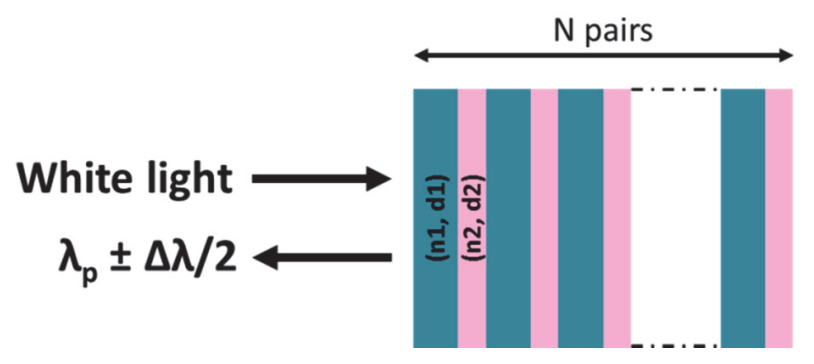

Fig. A 4.1 Sketch of a DBR stack showing the directions of the incident and reflected light. The incident light has a broad wavelength range (therefore termed as 'white'), whereas the reflected light has the DBR's design wavelength $\left(\lambda_{\mathrm{p}}\right)$ and bandwidth $(\Delta \lambda)$.

The sketch of an ideal DBR is shown in Fig. A 4.1. Pairs of alternating indices $\left(n_{1}\right.$, $\left.n_{2}\right)$ and thicknesses $\left(d_{1}, d_{2}\right)$ are indicated. The complete stack is composed of $N$ such pairs. The DBR has a design wavelength $\left(\lambda_{\mathrm{p}}\right)$ and a bandwidth $(\Delta \lambda)$. The bandwidth is conveniently expressed in units of frequency $(\Delta f)$. The reflectivity of the DBR is expressed as $R_{\mathrm{p}}$. The expressions for $R_{\mathrm{p}}, \lambda_{\mathrm{p}}$ and $\Delta f$ are given by Eqn. A 4.1 and Eqn. A 4.2, respectively ${ }^{42-43}$. The contrast of the refractive index between the two layers $(\Delta n)$ is given by Eqn. A 4.3.

$$
\begin{array}{cc}
R_{p}=\left[\frac{1-\left(n_{1} / n_{2}\right)^{2 N}}{1+\left(n_{1} / n_{2}\right)^{2 N}}\right]^{2} & \text { Eqn. A 4.1 } \\
\lambda_{p}=4 n_{1} d_{1}=4 n_{2} d_{2} \quad \Delta f=\frac{c}{\lambda_{p}} \frac{4}{\pi} \sin ^{-1}\left(\frac{n_{2}-n_{1}}{n_{2}+n_{1}}\right) & \text { Eqn. A } 4.2 \\
\Delta n=\left(n 2^{2}-n_{1}^{2}\right) / 2 n 2^{2} & \text { Eqn. A } 4.3
\end{array}
$$

Monolithic DBRs are utilized as integrated with photodetectors (PD), light emitting diodes (LEDs), lasers, etc. For instance, in a LED-DBR combined device, the DBR improves the light extraction efficiency of the LED and tunes its emission spectrum ${ }^{44-45}$. In a PD-DBR device, the DBR collects and traps the incident light and enhances the bandwidth- 
efficiency product. This is beneficial if the PD has a narrow spectral window ${ }^{46-47}$. In lasers, DBRs are employed in the amplification cavity ${ }^{48}$. There, it accommodates for the occasional drift in the wavelength of emission due to process-induced variations ${ }^{49}$.

A 4.2 Methods to increase the $\boldsymbol{R}_{\boldsymbol{p}}$ of DBR: $R_{\mathrm{p}}$ is a crucial parameter of the DBR in the above-mentioned applications. To increase the $R_{\mathrm{p}}$, two approaches may be pursued as evident from Eqn. A 4.1. They are: (i) increasing $\left(n_{1} / n_{2}\right)$, and (ii) increasing $N$. Approach (i) is usually followed since increasing $N$ induces defects, impurities, strain and surface roughness in the DBR stack which effectively reduce the $R_{\mathrm{p}}{ }^{50-51}$. A smaller $N$ also shortens the fabrication time and device dimensions.

To increase the $\left(n_{1} / n_{2}\right)$ ratio, combinations of various group III-Nitrides (e.g., AlN, $\mathrm{GaN}, \mathrm{InN}$ and their alloys) have been utilized due to contrasts in their refractive indices ${ }^{50-51}$. However, for applications demanding high $R_{\mathrm{p}}$, their combinations may still be unable to provide the desired $R_{\mathrm{p}}$. This would demand increasing $N$ at the cost of the above-mentioned short-comings.

A 4.3 Can AlN-GaCN outperform AlN-GaN in DBRs? The AlN-GaCN stack provides a higher $\Delta n$ compared to $\mathrm{AlN}-\mathrm{GaN}$ (or $\mathrm{AlN}-\mathrm{Al}_{\mathrm{x}} \mathrm{Ga}_{1-\mathrm{x}} \mathrm{N}$ ) due to the higher contrast of refractive indices (Eqn. A 4.3). For example, using the ALD (Al)GaN layers, the highest $\Delta n$ achievable for the $\mathrm{AlN}-\mathrm{GaN}$ combination is $8.4 \%$ at the dispersion-free region of $1500 \mathrm{~nm}$. On the contrary, $\Delta n$ for the $\mathrm{AlN}-\mathrm{Ga}_{0.66} \mathrm{C}_{0.10} \mathrm{~N}_{0.24}$ combination can be increased by up to $16.8 \%$ at the same wavelength. Even for the lowest-C-containing $\mathrm{GaCN}\left(\mathrm{Ga}_{0.66} \mathrm{C}_{0.03} \mathrm{~N}_{0.31}\right)$ in combination with AlN, $\Delta n$ can be improved to $12.8 \%$.

However, the superior refractive indices of the $\mathrm{GaCN}$ layers come with the cost of high levels of optical absorption (recall Fig. 4.9 b). Considering this factor is essential for the DBR design. Therefore, the following discussion leads to identifying a spectral window where $\mathrm{GaCN}$ can potentially outperform GaN as DBR material, in combination with AlN.

For absorbing layers, the expression for $R_{\mathrm{p}}$ becomes more elaborate (Eqn. A 4.4) as derived from expressions in reference ${ }^{52}$, compared to Eqn. A 4.1. $k_{1}$ and $k_{2}$ are the extinction coefficients of the two DBR materials. The reflectivity in the presence of absorption is denoted by $R_{\mathrm{p} \_\mathrm{k}} R_{\mathrm{p}}$ represents the same without considering absorption (i.e., Eqn. A 4.1). From Eqn. A 4.4, it is apparent that $R_{\mathrm{p}_{-} \mathrm{k}}$ decreases as $k_{1}$ and $k_{2}$ increases. 


$$
R_{p_{-} k}=R_{p}-\frac{\pi}{2} R_{p}\left(1+R_{p}\right)\left[\left(\frac{k_{1}}{n_{1}}+\frac{k_{2}}{n_{2}}\right)\left(\frac{1 / n_{2}}{1-n_{1} / n_{2}}\right)\left(\frac{\left[1+n_{1}{ }^{2}\left(n_{1} / n_{2}\right)^{N-1}\right]\left[1-\left(n_{1} / n_{2}\right)^{N}\right]}{1+\left(n_{1} / n_{2}\right)^{2 N}}\right)\right]
$$

Eqn. A 4.4

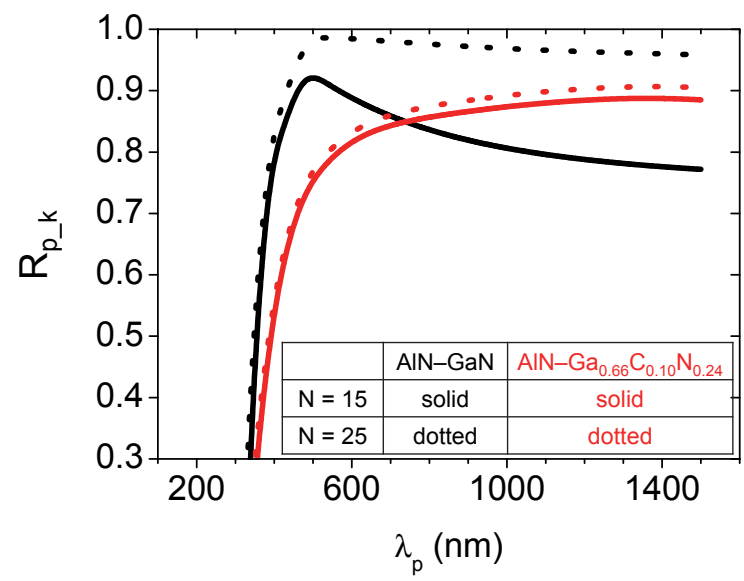

(a)

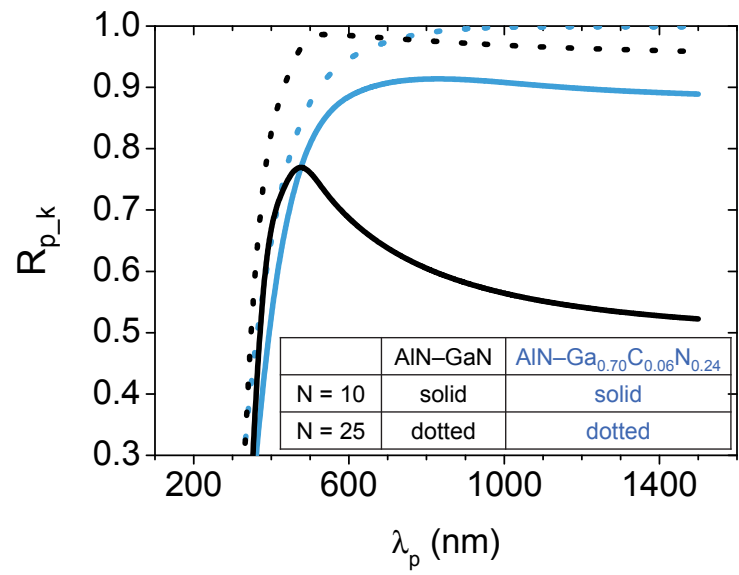

(b)

Fig. A 4.2 Simulated variation of $R_{\mathrm{p} \_}$with $\lambda_{\mathrm{p}}$ for (a) $\mathrm{AlN}-\mathrm{GaN}$ and $\mathrm{AlN}-\mathrm{Ga}_{0.66} \mathrm{C}_{0.10} \mathrm{~N}_{0.24} \mathrm{DBRs}$ with $N=15$ and 25. (b) AlN-GaN and $\mathrm{AlN}-\mathrm{Ga}_{0.70} \mathrm{C}_{0.06} \mathrm{~N}_{0.24}$ DBRs with $N=10$ and 25.

Fig. A 4.2 a compares the spectral variation of $R_{\mathrm{p}_{-} \mathrm{k}}$ for $\mathrm{AlN}-\mathrm{GaN}$ and $\mathrm{AlN}-$ Ga0.66 $\mathrm{C}_{0.10} \mathrm{~N}_{0.24}$ DBRs, assuming 15 and 25 pairs ${ }^{*}$. For $N=15$, the $\mathrm{AlN}-\mathrm{Ga} 0.66 \mathrm{C}_{0.10} \mathrm{~N}_{0.24}$ combination outperforms the $\mathrm{AlN}-\mathrm{GaN}$ combination only beyond $750 \mathrm{~nm}$. Increasing $N$ to 25 causes a significant increase of $R_{\mathrm{p}_{-} \mathrm{k}}$ for the AlN-GaN DBR but hardly any improvement for the $\mathrm{AlN}-\mathrm{Ga}_{0.66} \mathrm{C}_{0.10} \mathrm{~N}_{0.24} \mathrm{DBR}$.

* Since the $\mathrm{Ga}_{0.66} \mathrm{C}_{0.10} \mathrm{~N}_{0.24}$ layer exhibited the highest absorption (recall Fig. 4.9 b), it is selected as the worst-case example for GaCN-based DBRs. The pair numbers are chosen arbitrarily. 
The $\mathrm{GaCN}$ layers prepared in this work exhibit both high $n$ and $k$; the latter dominant especially at the shorter wavelengths (recall Fig. 4.9 a and b). In order to maximize $R_{\mathrm{p}_{-} \mathrm{k}}$, raising $N$ increases the contributions of both $n$ and $k$. Whereas the contribution of the former has a positive effect on $R_{\mathrm{p}_{-} \mathrm{k}}$, that of the latter has an adverse effect. Therefore, it is expected that within a certain a spectral range, GaCN layers with specific compositions could potentially outperform GaN.

Fig. A 4.2 b compares the $R_{\mathrm{p}_{-} \mathrm{k}}$ of $\mathrm{AlN}-\mathrm{GaN}$ and $\mathrm{AlN}-\mathrm{Ga} 0.70 \mathrm{C}_{0.06} \mathrm{~N}_{0.24} \mathrm{DBRs}$ for $N=10$ and 25. For $N=25$, the performances of the two DBRs are almost comparable. However, for $N=10$, GaCN clearly outperforms GaN from wavelengths as low as $475 \mathrm{~nm}$. Moreover, the GaCN-based DBR is able to sustain a high $R_{\mathrm{p}_{-} \mathrm{k}}$ of $\sim 0.9$ throughout the spectral range. This shows that this and other $\mathrm{GaCN}$ layers (with various compositions) can also be utilized in DBRs following a careful consideration of their C-content and the DBR $N$ number.

A 3.4 Conceptualized LED-DBR device utilizing GaCN': The conceptual LED-DBR integrated device is shown in Fig. A 4.3. The device is fabricated in a cavity in a Si substrate, with the LED (shown by the box) contained within the AIN-GaCN DBR. In the presence of the DBR, light propagating into the substrate is reflected back and re-emitted as indicated by the ray to the right. This reduces the light loss and enhances the efficiency of the LED. Besides, the DBR may also be used to tune the emitted wavelength according to its $\lambda_{\mathrm{p}}$ value.

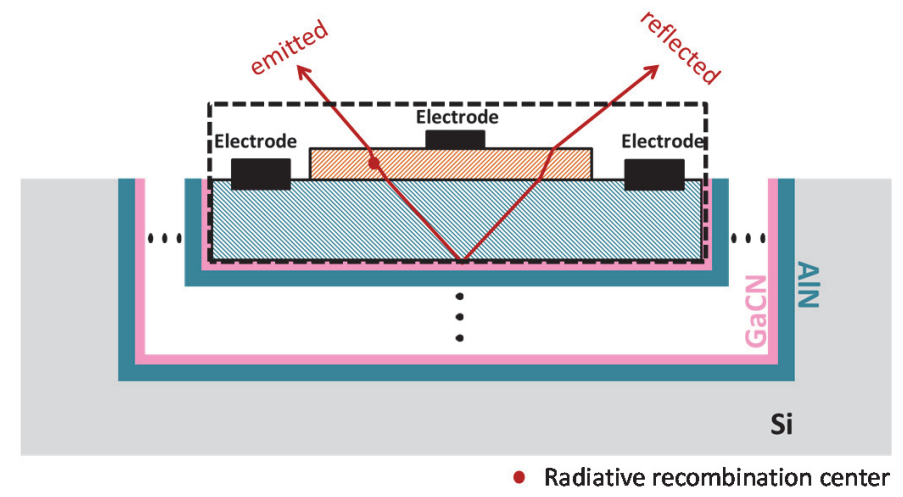

Fig. A 4.3 Sketch of a conceptual LED-DBR device integrated in Si. The LED is enclosed by the box. The red dot represents the radiative recombination center in the LED. The DBR comprises of a stack of alternating $\mathrm{AlN}$ and $\mathrm{GaCN}$ layers.

$\dagger$ Equally contributed by dr. Satadal Dutta, currently at the Department of Precision and Microsystems Engineering, TU Delft (The Netherlands). 
In an ALD reactor, the fabrication of such a DBR seems feasible in one process, i.e., without vacuum break, by switching the precursors. ALD ensures a high level of conformality, thickness control and low surface roughness of the DBR. Besides, the composition of $\mathrm{GaCN}$ (hence, $n$ and $k$ ) can be tuned by adjusting the precursors' partial pressure and the deposition temperature. The trade-off, though, is the low GPC in ALD which must be considered if employing thick layers. 


\section{Thermal ALD of polycrystalline GaN}

${ }^{*}$ This chapter is based on:

S. Banerjee, A. A. I. Aarnink, D. J. Gravesteijn and A. Y. Kovalgin, The Journal of Physical Chemistry $C$ (under review). A part of its contents was presented at the ALD 2018 Conf. (Incheon, July 2018).

Prior to arriving at the reported results and conclusions, some important early insights were obtained when experiments were performed in a pulsed-deposition scheme, but not necessarily conducive to ALD conditions. For example, the purge durations were kept short and the estimation of the ALD window was not performed. Those initial 'exploratory' results, although contributing significantly to the present understanding of the process, have not been included in this chapter. The interested reader may find the same in reference $\left({ }^{1}\right)$. 


\subsection{Introduction}

This chapter introduces the technique to deposit poly-GaN films by a novel low temperature thermal ALD process, using the industrially-relevant TMG and $\mathrm{NH}_{3}$ precursors. The ALD occurs between $375-425^{\circ} \mathrm{C}$ and yields a GPC as high as $0.1 \mathrm{~nm} /$ cycle. Carbon free, polycrystalline films with sub-10 $\mathrm{nm}$ crystalline domains are deposited. The approach relies on the speculated existence of a novel chemical species: the so-called TMG: $\mathrm{NH}_{3}$ surface adduct, and its conversion into GaN. The formation of the surface adduct is enhanced by the partial pressure of $\mathrm{NH}_{3}$, and its conversion is enhanced by the duration of the $\mathrm{NH}_{3}$ pulse. These two parameters directly affect the growth per cycle (GPC) of the layers.

Although GaN ALD processes using TMG and $\mathrm{NH}_{3}$ have been reported many times before (Section 5.2), almost every report concerns the use of plasma or other means of radical generation. Hardly any group could previously achieve a low temperature, purely thermal GaN ALD, which will be addressed in this chapter. Such a purely thermal ALD process has major advantages. First, the presence of ions and radiation in plasma may cause damage to the substrate or the growing film ${ }^{2}$. Second, involving radicals may limit the filmconformality, especially in high aspect ratio (HAR) structures due to their recombination tendency ${ }^{2-3}$. The same issue limits the application of radical-assisted ALD to only singlewafer reactors; scaling up to batch-reactors remains a challenge. A purely thermal ALD process resolves these issues.

Section 5.2 reviews the previous ALD reports on GaN as a premise to this chapter. Section 5.3 proposes the GaN ALD growth model, based on a similar AIN ALD model reported in the literature. Section $\mathbf{5 . 4}$ looks at the accepted set of reactions occurring in metalorganic chemical vapor deposition (MOCVD) of GaN, focusses especially on the gasphase adduct pathway, and then identifies the similarities between the MOCVD reactions and the proposed ALD model. Section 5.5 deals with the GaN ALD kinetics. Section 5.6 reports the experimentally-obtained GPC variation with the $\mathrm{NH}_{3}$ pulse duration, the $\mathrm{NH}_{3}$ partial pressure, the deposition temperature, and the post- $\mathrm{NH}_{3}$ purge duration. Section 5.7 uses in-situ SE to further investigate the ALD process. Section 5.8 reports the material characterization of the GaN layers. Section 5.9 concludes the chapter.

\subsection{Review of previous GaN ALD reports}

For GaN ALD, metalorganic precursors (e.g., TMG and TEG) or halide-based inorganic Ga precursors (e.g., $\mathrm{GaCl}_{3}$ ) have been used, often utilizing $\mathrm{NH}_{3}$ or $\mathrm{N}_{2}-\mathrm{H}_{2}$ as the nitrogen source ${ }^{4-14}$. The challenge of ALD by purely thermal means (i.e., without using additional activation techniques) lies in the activation of the nitrogen precursor, which is 
chemically very stable. For example, $\mathrm{NH}_{3}$ starts to dissociate on the surface only beyond $600{ }^{\circ} \mathrm{C}^{15}$.

Thermal ALD from $\mathrm{GaCl}_{3}$ and $\mathrm{NH}_{3}$ has been reported to occur at high temperatures, exceeding $400{ }^{\circ} \mathrm{C}^{12-13}$. The halide precursor however exhibits several drawbacks, such as difficulty in delivery due to the low vapour pressure (demanding supply and gas-line heating), etching of the reactor walls from corrosive by-products (e.g., $\mathrm{HCl}$ ) and $\mathrm{Cl}$ incorporation into the growing film ${ }^{7}$.

On the contrary, ALD with TMG or TEG typically demands resorting to low temperatures $\left(<400{ }^{\circ} \mathrm{C}\right)^{4}, 7,9-10,16$, since these precursors tend to dissociate at higher temperatures, resulting in significant $\mathrm{Ga}$ - and $\mathrm{C}$-contamination in the layers ${ }^{17}$. In order to carry out the ALD at sub-dissociation temperature of the metalorganics, researchers resort to additional activation means, such as with plasma ${ }^{4,7,9}$, hot-filament ${ }^{11,18}$, or electron beam ${ }^{11}$, in order to dissociate the $\mathrm{N}$-precursor into radicals (at-H and $\mathrm{NH}_{\mathrm{x}}$, where $x=0-2$ ). Such radical-enhanced ALD lowers the process temperature and enables an ALD window for GaN. To the best of our knowledge, there exists two earlier publications on thermal GaN ALD from TMG and $\mathrm{NH}_{3}$ precursors ${ }^{19-20}$. However, both are high temperature $\left(>500{ }^{\circ} \mathrm{C}\right)$ processes, where the authors primarily focussed on characterizing the layer properties, without detailed investigation into the growth mechanism or demonstration of (the existence of) an ALD window.

\subsubsection{Novelty of the proposed GaN ALD process}

We propose a low temperature chemical route, perhaps previously overlooked by the GaN ALD community, that can be used to deposit poly-GaN films from TMG and $\mathrm{NH}_{3}$, without cracking the $\mathrm{NH}_{3}$ into radicals. This novel ALD route was identified from (i) the previously-reported ALD mechanism of AlN films (using $\mathrm{NH}_{3}$ and the analogous-to-TMG, TMA), and (ii) supported by the reported chemical models of GaN growth in MOCVD. GaN growth through this novel ALD route occurred with a GPC as high as $0.1 \mathrm{~nm} /$ cycle at $400{ }^{\circ} \mathrm{C}$ temperature.

Similar to the TMA: $\mathrm{NH}_{3}$ surface adduct species that participates in AlN ALD (Section 5.3.1), and equivalent to the $\mathrm{TMG}^{\mathrm{N}} \mathrm{NH}_{3}$ gas-phase adduct species known for GaN MOCVD reactions (Section 5.4), we hypothesize the existence of the TMG: $\mathrm{NH}_{3}$ surface adduct as necessary species to enable GaN ALD in a purely thermal mode. 


\subsection{Surface reactions leading to GaN ALD}

\subsubsection{Reported mechanism of thermal AIN ALD}

The mechanism of thermal ALD of AIN from TMA and $\mathrm{NH}_{3}$ has been widely reported $^{20-22}$, unlike that of $\mathrm{GaN}$. On a $\mathrm{SiO}_{2}$ substrate, TMA chemisorbs as monomethyl aluminium $\left(\mathrm{Al}-\mathrm{CH}_{3}\right)$ to hydroxyl $(-\mathrm{OH})$ or siloxane $(\mathrm{Si}-\mathrm{O}-\mathrm{Si})$ sites by the loss of two of its methyl $\left(-\mathrm{CH}_{3}\right)$ groups ${ }^{21}$. The subsequently dosed $\mathrm{NH}_{3}$ physisorbs on the $\mathrm{Al}-\mathrm{CH}_{3}$ unit, resulting in the TMA: $\mathrm{NH}_{3}$ surface adduct: each adduct unit therefore contains an $\mathrm{Al}$ atom chemisorbed to the substrate, a $-\mathrm{CH}_{3}$ group bonded to the $\mathrm{Al}$, and a $\mathrm{NH}_{3}$ molecule physisorbed to the $\mathrm{Al}^{22}$. The chemical origin of the surface adduct is attributed to TMA and $\mathrm{NH}_{3}$ being strong Lewis acid and base, respectively ${ }^{22}$. Therefore, the adduct forms already at low temperatures on the surface $\mathrm{e}^{22}$ or in the gas-phase ${ }^{23}$, through a strong electrostatic force of attraction between $\mathrm{Al}$ and $\mathrm{N}$.

The surface adduct, however, does not remain intact when heat is applied. Typically beyond $300{ }^{\circ} \mathrm{C}$, the physisorbed $\mathrm{NH}_{3}$ of one adduct unit hydrogenates the $-\mathrm{CH}_{3}$ group of a neighbouring adduct unit, eliminating a $\mathrm{CH}_{4}$ and concurrently forming a $\mathrm{Al}-\mathrm{NH}_{2}-\mathrm{Al}$ linkage between the two adduct units ${ }^{22}$. This reaction is self-limiting in nature. The formation of $\mathrm{Al}-\mathrm{NH}_{2}-\mathrm{Al}$ linkages over the entire surface implies a monolayer growth of AlN. The subsequently pulsed TMA chemisorbs on the $-\mathrm{NH}_{2}-$ unit of the linkage, reterminating the surface with $-\mathrm{CH}_{3}$ groups, and forming half of the next monolayer ${ }^{22}$. Such re-generative feature of the surface reactions between TMA and $\mathrm{NH}_{3}$ is proposed to be a viable chemical pathway in the thermal ALD of AlN films ${ }^{22}$.

\subsubsection{Proposed mechanism of thermal GaN ALD}

Unlike the TMA: $\mathrm{NH}_{3}$ surface adduct, the experimental evidence of the TMG: $\mathrm{NH}_{3}$ surface adduct (and its role in GaN ALD) has never been reported. This could be partly because of the nature of interaction of TMG with the substrate $\left(\mathrm{Si}, \mathrm{SiO}_{2}\right)$, which is somewhat different than for TMA. Whereas TMA can chemisorb onto these substrates, and further remains stable at the ALD temperatures of $\mathrm{AlN}\left(300-400{ }^{\circ} \mathrm{C}^{24}\right)$, TMG starts to dissociate on the substrates roughly beyond $150{ }^{\circ} \mathrm{C}$, successively eliminating its $-\mathrm{CH}_{3}$ groups ${ }^{25-26}$. At our deposition temperatures $\left(350{ }^{\circ} \mathrm{C}\right.$ and beyond), whether TMG still possesses one or more $-\mathrm{CH}_{3}$ groups is therefore questionable. However, based on the experimental results (Section 5.6 - 5.8 and Chapter 6, Section 6.4), we speculate that after several ALD cycles $(250-300)$ on $\mathrm{Si}$ or $\mathrm{SiO}_{2}$, these substrates can accommodate the necessary coverage of $-\mathrm{CH}_{3}$-terminated sites to support the GaN ALD. 
Equivalent to AlN ALD, a growth model for GaN ALD is proposed in Fig. 5.1. Post TMG pulse, $\mathrm{NH}_{3}$ dose to the $-\mathrm{CH}_{3}$-terminated surface leads to the formation of TMG: $\mathrm{NH}_{3}$ surface adduct. Aided thermally, the neighbouring adduct units convert into a $\mathrm{Ga}-\mathrm{NH}_{2}-\mathrm{Ga}$ linkage. During the $\mathrm{NH}_{3}$ pulse, such $\mathrm{Ga}-\mathrm{NH}_{2}-\mathrm{Ga}$ units (abbreviated henceforth as "- $\mathrm{NH}_{2}-$-") concurrently form at different locations on the substrate, laterally propagating and increasing the surface coverage, ideally leading to the formation of a GaN monolayer.

Although the existence of the $\mathrm{TMG}: \mathrm{NH}_{3}$ surface adduct is yet to be experimentally verified, a theoretical study on $\mathrm{GaN} \mathrm{MOCVD}^{27}$ in fact makes use of the same surface adduct (the so-called "TCOM1(s)" species ${ }^{27}$ ) to model the GaN formation, by one out of the several explored pathways. Namely, the steps consist of (i) adsorption of TMG to a nitrogen site, (ii) adsorption of $\mathrm{NH}_{3}$ to the TMG, forming the surface adduct, and (iii) extraction of $\mathrm{CH}_{4}$ molecules from the adduct leading to $\mathrm{GaN}$ formation. The possibility of the existence of the surface adduct is also supported by an experimental study on the reactions between TMG and $\mathrm{NH}_{3}$ in MOCVD, which concludes that the formation of adducts occur 'on or near' the GaN growth surface ${ }^{28}$.

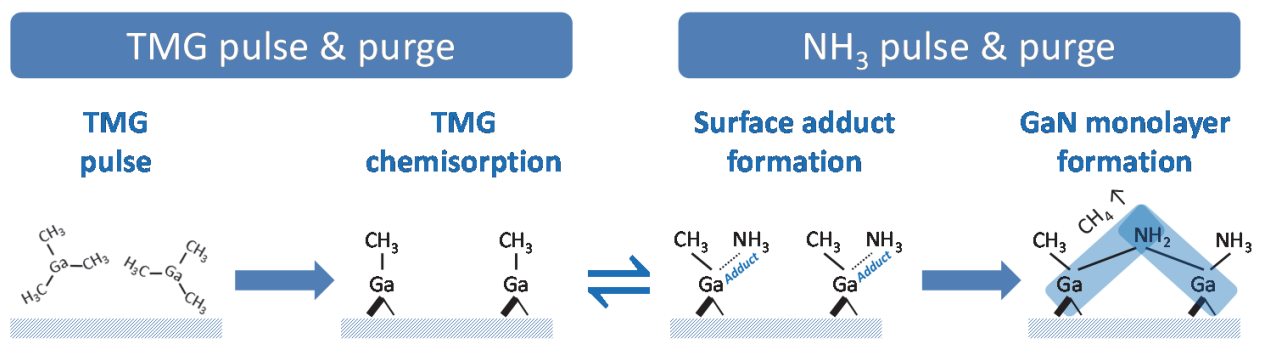

Fig. 5.1 Proposed mechanism of thermal GaN ALD through (i) TMG chemisorption, (ii) reversible formation of the TMG: $\mathrm{NH}_{3}$ surface adduct, and (iii) irreversible conversion of neighbouring surface adducts into a $\mathrm{Ga}-\mathrm{NH}_{2}-\mathrm{Ga}$ linkage (shaded in blue), signifying the formation of a $\mathrm{GaN}$ unit. Propagation of the linkage on the surface is expected to ultimately result in a monolayer of GaN. (To note: the TMG chemisorption schematic only serves to show that a $-\mathrm{CH}_{3}$ terminated surface can be expected after the TMG pulse, after several ALD cycles. The exact nature of TMG chemisorption is beyond the scope of this chapter.)

\subsection{GaN MOCVD reactions from TMG and $\mathrm{NH}_{3}$ precursors}

In the absence of reports on thermal GaN ALD mechanism, we shall briefly overview the reactions occurring in GaN MOCVD, where the existence of the TMG: $\mathrm{NH}_{3}$ gas-phase adduct is reported, and then compare them with the surface adduct ALD model.

The MOCVD of epitaxial GaN from TMG and $\mathrm{NH}_{3}$ is performed at substrate temperatures typically exceeding $900{ }^{\circ} \mathrm{C}$; the accepted gas-phase and surface reactions are 
shown in Fig. 5.2. The pathway leading to the epitaxial GaN growth is shown by the solid arrows. In this pathway, TMG dissociates into smaller units such as dimethylgallium $\left(\mathrm{Ga}-\left(\mathrm{CH}_{3}\right)_{2}\right)$ and monomethylgallium $\left(\mathrm{Ga}-\mathrm{CH}_{3}\right)$ already in gas-phase ${ }^{27,29-30}$. As these diffuse towards the substrate, their further dissociation leads to the formation of clusters of Ga.

In parallel, $\mathrm{NH}_{3}$ dissociates on the substrate forming $\mathrm{NH}_{\mathrm{x}}(x=0-2)$ and at- $\mathrm{H}$ radicals ${ }^{31-32}$. These radicals recombine with the Ga clusters (and/or di-, tri-methylgallium (DMG, TMG)) on the substrate. At the high substrate temperature, the surface reaction products possess sufficient mobility to migrate to energetically-favourable surface sites, leading to crystalline GaN growth ${ }^{33}$.

A set of gas-phase reactions may also occur away from the substrate, at lower (a few-hundred-degrees) temperatures, as indicated by the dashed arrows (Fig. 5.2). The first reaction is the formation of the $\mathrm{TMG}_{\mathrm{NH}} \mathrm{NH}_{3}$ gas-phase adduct ${ }^{34-35}$. The adduct tends to polymerize into larger amidic clusters, having the form: $\left(\left(\mathrm{CH}_{3}\right)_{2}-\mathrm{Ga}-\left(\mathrm{NH}_{2}\right)\right)_{\mathrm{n}}$ or $\left(\left(\mathrm{CH}_{3}\right)_{3}-\mathrm{Ga}:\left(\mathrm{NH}_{3}\right)_{\mathrm{n}}\right)^{36-37}$. These clusters either migrate towards the substrate or away from the substrate, depending on the flow profile.

Migration towards the substrate is highly undesired in MOCVD growth as it results in polycrystalline $\mathrm{GaN}$ formation due to the poor surface mobility of the clusters ${ }^{30}$. Moreover, they may also introduce carbon contamination in the layer. Migration away from the substrate leads to their further polymerization, eventually forming particles up to several hundred nanometers in size ${ }^{38}$. The particle formation can significantly deplete the precursor flux, reducing the epi-GaN growth rate $^{38}$. Due to these issues, the adduct pathway is considered to be parasitic in GaN MOCVD, and therefore to be avoided ${ }^{38}$. This pathway has however been studied in detail to understand, and therefore minimize its influence.

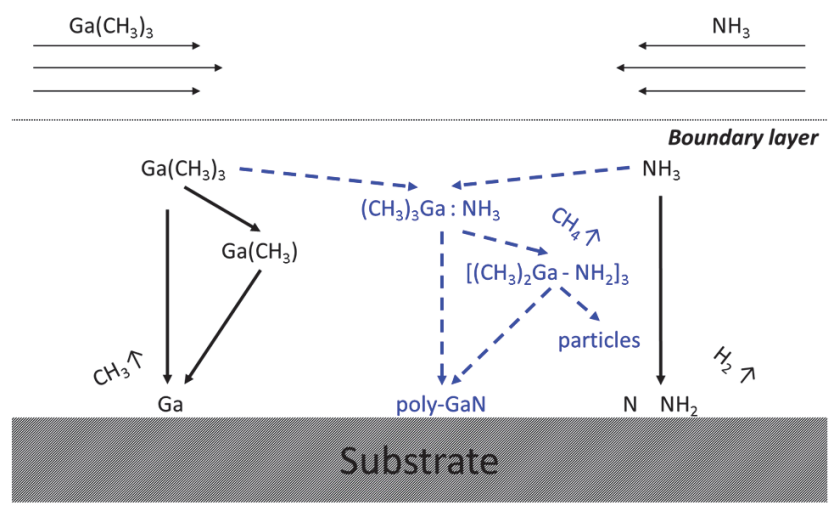

Fig. 5.2 The simplified reaction schematic in GaN MOCVD, as compiled from several growth models (see text for references). The epi-GaN and adduct pathways are shown by solid and dashed arrows, respectively. 


\subsubsection{The adduct pathway in more detail}

Several experimental ${ }^{34-37,39}$ and theoretical ${ }^{40-44}$ reports address the several reactions occurring in the adduct pathway. The electrostatic bonding between the Lewis acid (TMG) and the Lewis base $\left(\mathrm{NH}_{3}\right)$, i.e., forming the adduct, occurs with zero activation energy $\left(E_{\mathrm{A}}\right)^{45}$, and with an enthalpy of formation $(\Delta H)$ of $\sim-16 \mathrm{kcal} / \mathrm{mol}^{46}$ (Fig. 5.3 a).

The electrostatic bond is however not strong (i.e., $\Delta H$ is low), making the reaction reversible in nature ${ }^{46}$ (Reaction 5.1). This in fact originates due to a modest electronegativity difference between $\mathrm{Ga}(1.81)$ and $\mathrm{C}$ (2.55), which causes the $\mathrm{Ga}-\mathrm{CH}_{3}$ bonds in the adduct to be less ionic, and therefore makes it difficult for $\mathrm{NH}_{3}$ to form an electrostatic bond with Ga. This in turn implies that the rate of the adduct formation can be suppressed by decreasing the precursors' partial pressure, and vice versa.

$$
\mathrm{Ga}\left(\mathrm{CH}_{3}\right)_{3}+\mathrm{NH}_{3} \leftrightharpoons \mathrm{Ga}\left(\mathrm{CH}_{3}\right)_{3}: \mathrm{NH}_{3}
$$

\section{Reaction 5.1}

(To compare with the gas-phase $\mathrm{Al}\left(\mathrm{CH}_{3}\right)_{3}: \mathrm{NH}_{3}$ adduct formation (Fig. $5.3 \mathrm{~b}$ ), the electrostatic bond is stronger $\left(\Delta H=-27 \mathrm{kcal} / \mathrm{mol}^{36}\right)$. This is due to the larger electronegativity difference between $\mathrm{Al}(1.61)$ and $\mathrm{C}(2.55)^{47}$. This shifts the equilibrium towards the Al-adduct formation ${ }^{36}$.)

Further, the $\mathrm{Ga}\left(\mathrm{CH}_{3}\right)_{3}: \mathrm{NH}_{3}$ adduct can also irreversibly form the amidic $\left(\mathrm{CH}_{3}\right)-\mathrm{Ga}-$ $\mathrm{NH}_{2}$ unit, through an intra-adduct hydrogen transfer from $\mathrm{NH}_{3}$ to $-\mathrm{CH}_{3}$, liberating $\mathrm{CH}_{4}$ (Reaction 5.2) ${ }^{48}$. The $E_{\mathrm{A}}$ of this reaction is $\sim 31 \mathrm{kcal} / \mathrm{mol}^{36}$ (Fig. $5.3 \mathrm{a}$ ). The further reactions of the adduct pathway are not considered for brevity.

$$
\mathrm{Ga}\left(\mathrm{CH}_{3}\right)_{3}: \mathrm{NH}_{3} \rightarrow\left(\mathrm{CH}_{3}\right)-\mathrm{Ga}-\mathrm{NH}_{2}+\mathrm{CH}_{4} \uparrow
$$

\section{Reaction 5.2}

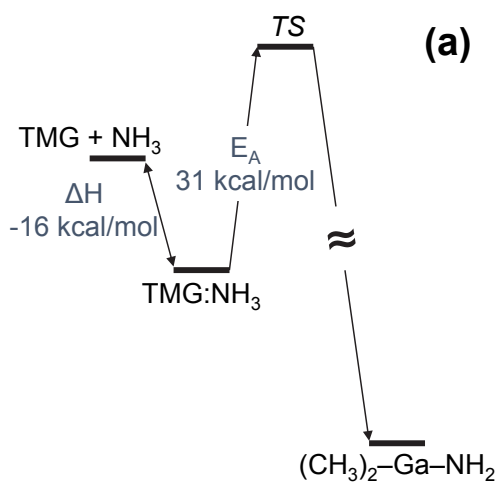

(b)

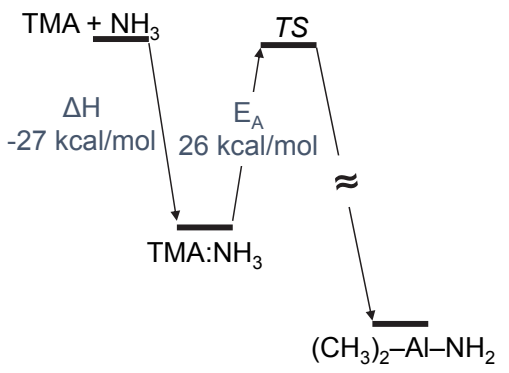

Fig. 5.3 Reaction energies for gas-phase (a) $\mathrm{TMG}-\mathrm{NH}_{3}$ and (b) $\mathrm{TMA}-\mathrm{NH}_{3}$ systems. TS denotes the transition states. 
The as-presented GaN ALD model can therefore be viewed as the representation of the MOCVD adduct pathway on a surface in a pulsed-precursor deposition scheme. The equivalent of the TMG: $\mathrm{NH}_{3}$ gas phase adduct is the as-hypothesized surface adduct. Similarly, the equivalent of the $\left(\mathrm{CH}_{3}\right)-\mathrm{Ga}-\mathrm{NH}_{2}$ amide is the $-\mathrm{NH}_{2}-$ surface linkage, the latter enabling a low temperature chemical route for GaN ALD.

\subsection{Modelling the GaN ALD kinetics}

The TMG: $\mathrm{NH}_{3}$ surface adduct formation is assumed to be reversible as its gasphase counterpart, because the same effects of electronegativity are expected to apply on the surface. The reversibility has also been theoretically utilized to model the GaN growth ${ }^{27}$. This implies that once physisorbed as adduct, the $\mathrm{NH}_{3}$ can desorb to the gas-phase before forming the $-\mathrm{NH}_{2}$ - linkage. However, if the linkage is already formed, then it is assumed to be irreversible.

The linkage forms from the reaction of $\mathrm{NH}_{3}$ group of one surface adduct unit with an adjacent $-\mathrm{CH}_{3}$ group of a chemisorbed TMG or that of the surface adduct species. The groups should also be oriented in the right manner to facilitate their interaction. For the TMA: $\mathrm{NH}_{3}$ surface adduct, such interactions have been reported ${ }^{22}$.

\subsubsection{TMG: $\mathrm{NH}_{3}$ surface adduct formation}

During a $\mathrm{NH}_{3}$ pulse of duration $t_{\mathrm{NH}}$, the adsorption of $\mathrm{NH}_{3}$ on the chemisorbedTMG sites (equivalently, the adduct surface coverage, $\theta$ ) can be expressed by the Langmuir adsorption-desorption kinetics ${ }^{49}$ (Eqn. 5.1). In this equation, $C_{\mathrm{NH} 3}$ is the concentration of $\mathrm{NH}_{3}$, and $k_{\mathrm{a}}$ and $k_{\mathrm{d}}$ are the rate constants of $\mathrm{NH}_{3}$ adsorption and desorption, respectively. Eqn. 5.1 indicates that $\theta$ increases with both $t_{\mathrm{NH} 3}$ and $C_{\mathrm{NH} 3}$; the latter can be related to the $\mathrm{NH}_{3}$ partial pressure $\left(P_{\mathrm{NH} 3}\right)$ through the relation: $C_{\mathrm{NH} 3}=P_{\mathrm{NH} 3} / k_{\mathrm{b}} T$, where $T$ is the deposition temperature.

$$
\theta\left(t_{\mathrm{NH} 3}\right)=k_{\mathrm{a}} C_{\mathrm{NH} 3} /\left(k_{\mathrm{a}} C_{\mathrm{NH} 3}+k_{\mathrm{d}}\right)\left[1-e^{-\left(k_{\mathrm{a}} C_{\mathrm{NH} 3}+k_{\mathrm{d}}\right) t_{\mathrm{NH} 3}}\right] \quad \text { Eqn. } 5.1
$$

The adduct coverage obtained from Eqn. 5.1 as a function of $t_{\mathrm{NH} 3}$ and $P_{\mathrm{NH} 3}$, after assuming chemically reasonable values of $k_{\mathrm{a}}\left(10^{-11} \mathrm{~cm}^{3} \mathrm{~s}^{-1}\right)$ and $k_{\mathrm{d}}\left(10^{6} \mathrm{~s}^{-1}\right)^{*}$, is shown in Fig. 5.4 a. As evident, $\theta$ stabilizes (indicating an equilibrium surface coverage) within a few

\footnotetext{
${ }^{*}$ For example, $k_{\mathrm{a}}$ and $k_{\mathrm{d}}$ of the gas-phase adduct formation are determined to be $10^{-10} \mathrm{~cm}^{3} \mathrm{~s}^{-1}$ and $10^{8} \mathrm{~s}^{-1}$, respectively, from parameters reported in reference $\left({ }^{60}\right)$.
} 
microseconds of $t_{\mathrm{NH} 3}$. Changing the values of $k_{\mathrm{a}}$ and $k_{\mathrm{d}}$ over several orders of magnitude affects the time to reach the equilibrium coverage. For example, $k_{\mathrm{a}}$ and $k_{\mathrm{d}}$ of $10^{-18} \mathrm{~cm}^{3} \mathrm{~s}^{-1}$ and $10^{-1} \mathrm{~s}^{-1}$, respectively, result in 10-20 s, instead of microseconds. Such rate constants are however hardly physical. Furthermore, $\theta$ strongly depends on $P_{\mathrm{NH} 3}$, gradually approaching 1 at reasonably high $P_{\mathrm{NH} 3}$.
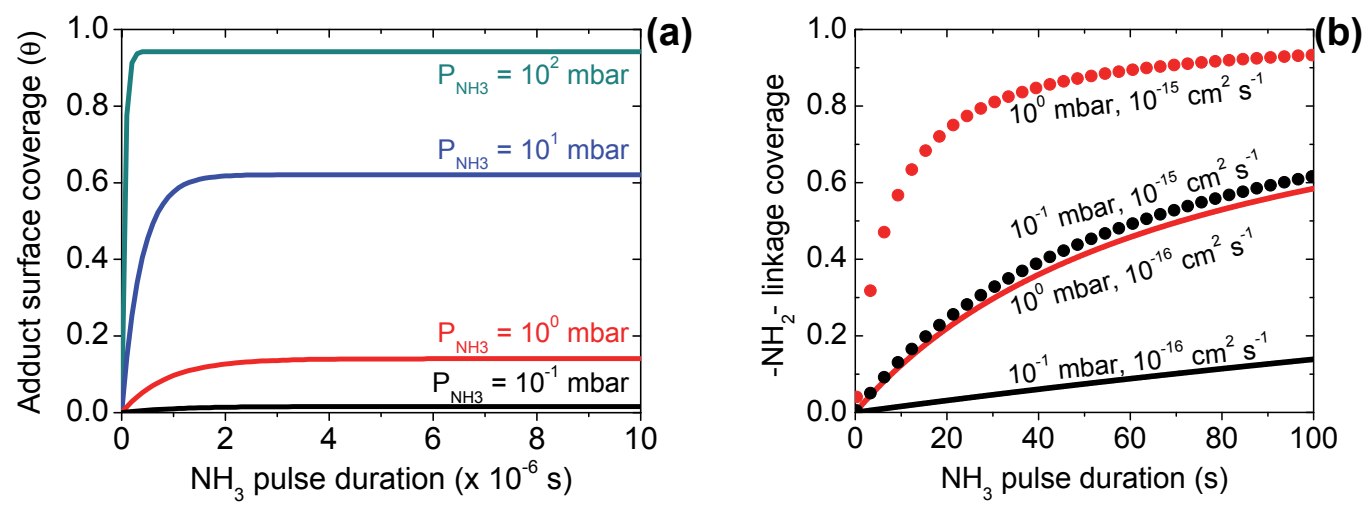

Fig. 5.4 The simulated variation of (a) the adduct surface coverage $(\theta)$ with the $\mathrm{NH}_{3}$ pulse duration $\left(t_{\mathrm{NH} 3}\right)$, under several $\mathrm{NH}_{3}$ partial pressures $\left(P_{\mathrm{NH} 3}\right)$; (b) surface coverage of $-\mathrm{NH}_{2}-$ linkage with $t_{\mathrm{NH}}$, under $10^{0}$ mbar (in red) and $10^{-1}$ mbar (in black) $P_{\mathrm{NH} 3}$, at $k_{\mathrm{GaN}}$ of $10^{-16} \mathrm{~cm}^{2} \mathrm{~s}^{-1}$ (solid) and $10^{-15} \mathrm{~cm}^{2} \mathrm{~s}^{-1}$ (dotted).

\subsubsection{Ga-NH$-\mathrm{Ga}$ linkage formation}

Since the surface adduct formation is assumed to be reversible, during a $\mathrm{NH}_{3}$ pulse the physisorbed $\mathrm{NH}_{3}$ can either desorb from the TMG site or irreversibly form the $-\mathrm{NH}_{2}-$ linkage. In the first case, the TMG site is free to accept a new $\mathrm{NH}_{3}$ molecule. In the second case, this site can be excluded from participating in further reactions during this particular $\mathrm{NH}_{3}$ pulse.

These considerations were applied in a Mathcad code (Appendix 5), for simulating the $-\mathrm{NH}_{2}-$ coverage as a function of $t_{\mathrm{NH} 3}$ and $P_{\mathrm{NH} 3}$ (Fig. 5.4 b). Since the rate constant $\left(k_{\mathrm{GaN}}\right)$ for the adduct to $-\mathrm{NH}_{2}$ - conversion is not known empirically, it was varied between $10^{-15}$ and $10^{-16} \mathrm{~cm}^{2} \mathrm{~s}^{-1}$, as it yielded time scales of the same order as experimentally obtained for GPC (see Fig. 5.5 a). As Fig. 5.4 b shows, if $t_{\mathrm{NH} 3}$ is long (up to several minutes), all the surface adduct sites can be eventually converted into $-\mathrm{NH}_{2}-$ linkages. A complete $-\mathrm{NH}_{2}-$ coverage implies the formation of a $\mathrm{GaN}$ monolayer.

Fig. 5.4 a and b further reveals the strong pressure dependence of the GaN ALD process. If the ALD is performed at low $P_{\mathrm{NH} 3}$, this would also result in low $\theta$ (Fig. 5.4 a). In 
such situation, only a very long $t_{\mathrm{NH} 3}$ would eventually convert all the adduct sites into $-\mathrm{NH}_{2}-$ linkages. On the contrary, ALD performed under high $P_{\mathrm{NH} 3}$ would already initially result in large $\theta$, therefore requiring a shorter $t_{\mathrm{NH} 3}$ to achieve a high $-\mathrm{NH}_{2}-$ coverage. In the next section, we shall experimentally validate this behaviour.

\subsection{The GaN ALD window}

\subsubsection{Variation of GPC with $t_{\mathrm{NH} 3}, P_{\mathrm{NH} 3}$ and $T$}

Fig. 5.5 a shows the variation of the GPC with $t_{\mathrm{NH} 3}$ and $P_{\mathrm{NH} 3}$, monitored by in-situ SE. The generic ALD recipe can be found in the figure caption. Long Ar purges were kept in order to minimize possible gas-phase reactions. This is important since the pressure is significantly higher than typically used for ALD; so the occurrence of gas-phase reactions can be otherwise expected. Besides, the Ar flow rate ensured 3 times change of the reactor volume every second.

From Fig. 5.5 a, one clearly sees the strong dependence of GPC on both $t_{\mathrm{NH} 3}$ and $P_{\mathrm{NH} 3}$. Needless to mention, the latter is the most important feature of this ALD process (because pressure usually has no effect in most ALD processes). For every $P_{\mathrm{NH} 3}$, the GPC hardly shows saturation even at long $t_{\mathrm{NH} 3}(30 \mathrm{~s}, 60 \mathrm{~s})$; instead, it shows a slow increase. This can be explained by the ongoing surface reactions involving adduct and $-\mathrm{NH}_{2}-$ linkage formation, as long as the $\mathrm{NH}_{3}$ pulse continues (recall Fig. 5.4 b).
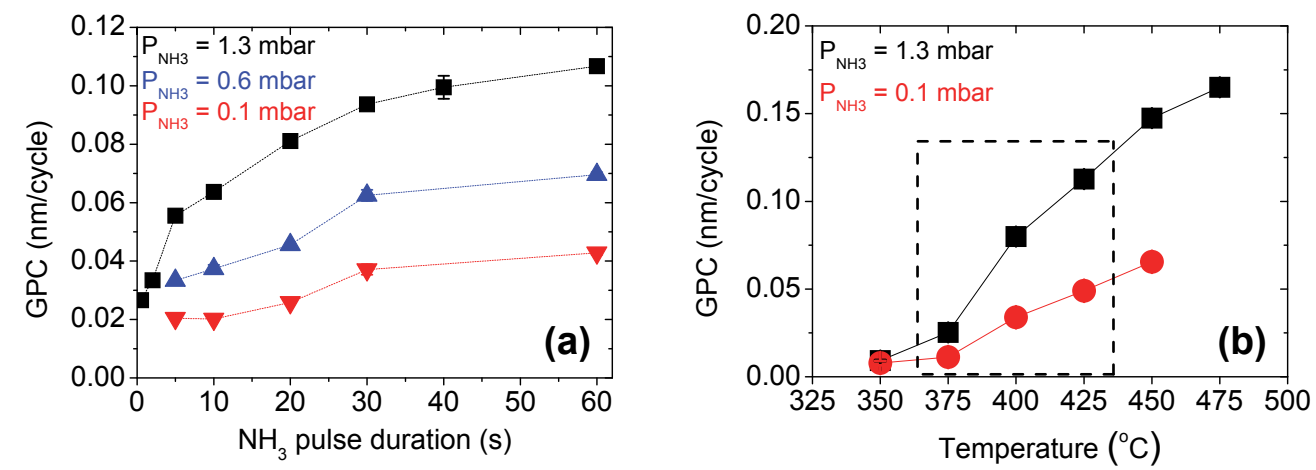

Fig. 5.5 The variation of GPC with (a) $\mathrm{NH}_{3}$ pulse duration $\left(t_{\mathrm{NH}}\right)$ and $\mathrm{NH}_{3}$ partial pressure $\left(P_{\mathrm{NH} 3}\right)$. The $P_{\mathrm{NH} 3}$ values correspond to a total reactor pressure of $10 \mathrm{mbar}$ (black), $5 \mathrm{mbar}$ (blue) and $1 \mathrm{mbar}$ (red). The generic ALD recipe was: $0.1 \mathrm{~s} \mathrm{TMG} / 30 \mathrm{~s} \mathrm{Ar} / 1-60 \mathrm{~s} \mathrm{NH}_{3} / 60 \mathrm{~s}$ Ar. All depositions were performed at $400{ }^{\circ} \mathrm{C}$. (b) GPC vs. deposition temperature $(T)$ and $P_{\mathrm{NH} 3}$. The ALD recipe was: $0.1 \mathrm{~s} \mathrm{TMG} / 30 \mathrm{~s} \mathrm{Ar} / 30 \mathrm{~s} \mathrm{NH}_{3} / 60 \mathrm{~s}$ Ar. The box indicates the temperature range where C-free $\mathrm{GaN}$ layers were deposited. 
Varying the TMG pulse duration at different $P_{\mathrm{NH} 3}(1,10$ mbar) hardly affects the GPC (Fig. 5.6 a). This indicates that even the shortest TMG pulse is sufficient to complete the surface coverage. Varying the post-TMG purge duration hardly affects the GPC as well (Fig. 5.6 b).

The variation of the GPC with the deposition temperature $(T)$ is shown in Fig. 5.5 b. Hardly any growth occurs at, or below $350{ }^{\circ} \mathrm{C}$. In terms of the ALD model, this indicates the inefficacy of the adduct to $-\mathrm{NH}_{2}-$ conversion at such temperatures. Beyond $350{ }^{\circ} \mathrm{C}$, the GPC steadily increases, with a higher rate at a higher $P_{\mathrm{NH} 3}$. At both pressures, no so-called 'temperature window' exists with a (near-to-)constant GPC. According to the XPS results (Section 5.8.4), the deposition of $\mathrm{C}$-free $\mathrm{GaN}$ is possible only within a narrow temperature range, approximately between 375 and $425^{\circ} \mathrm{C}$ (Fig. 5.5 b, boxed). Beyond $425{ }^{\circ} \mathrm{C}$, thermal dissociation of TMG results in an increasing amount of carbon and gallium in the layers; their content dependent on both temperature and pressure, as described in Chapter 4.
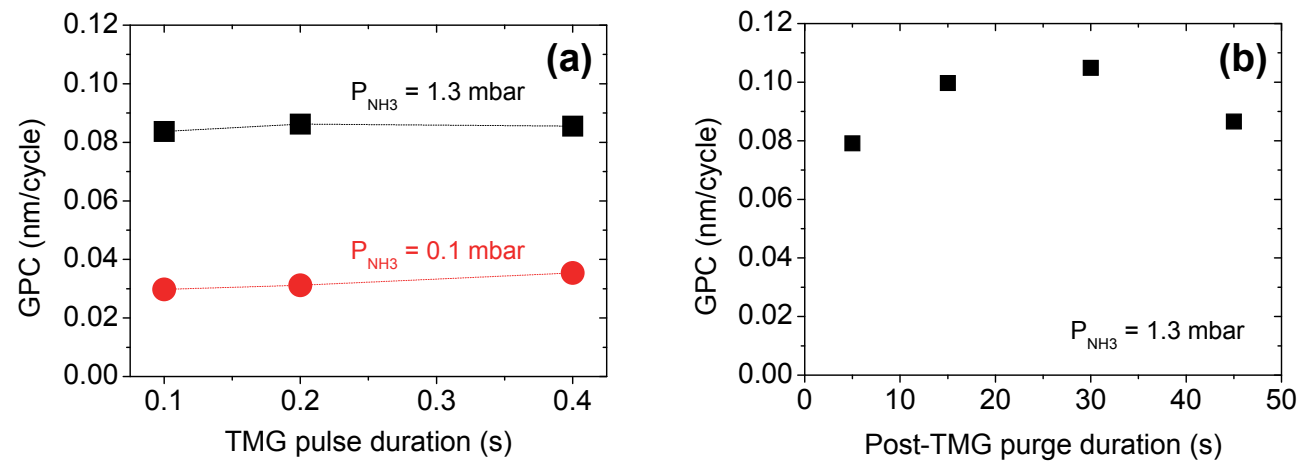

Fig. 5.6 Variation of GPC with (a) TMG pulse duration and $P_{\mathrm{NH} 3}$, performed with the generic ALD recipe: $0.1-0.4 \mathrm{~s}$ TMG / $30 \mathrm{~s} \mathrm{Ar} / 30 \mathrm{~s} \mathrm{NH}_{3} / 60 \mathrm{~s}$ Ar; (b) purge duration after the TMG pulse, with the generic ALD recipe: $0.1 \mathrm{~s}$ TMG / $5-45 \mathrm{~s} \mathrm{Ar} / 30 \mathrm{~s} \mathrm{NH}_{3} / 60 \mathrm{~s}$ Ar at 1.3 mbar $P_{\mathrm{NH} 3}$. Both the experiments were performed at $400{ }^{\circ} \mathrm{C}$.

\subsubsection{Anomalous variation of GPC with post- $\mathrm{NH}_{3}$ purge time}

Unexpectedly (and rather atypically for an ALD process), the GPC increases with the post-NH3 $\mathrm{NH}_{3}$ purge duration (Fig. 5.7 a). An opposite trend is usually observed in ALD; i.e., the GPC increases at short purge durations, due to gas-phase reactions additionally contributing to the growth. Beyond a certain purge duration, when the gas-phase components are sufficiently depleted, the GPC saturates. For instance, in case of GaN films deposited by $\mathrm{NH}_{3}$-plasma-assisted $\mathrm{ALD}^{4}$, the GPC indeed shows the expected behaviour (Fig. 5.7 b). However, for thermal ALD GaN, this is clearly not the case. 
We explain this anomalous behaviour by the high sticking coefficient of $\mathrm{NH}_{3}$ to the reactor surface made of stainless steel ${ }^{50}$. Onnink et al. ${ }^{51}$, studying the kinetics of $\mathrm{NH}_{3}$ removal from a cold-wall reactor by in-situ absorption spectroscopy, observed that $P_{\mathrm{NH} 3}$ indeed decayed slowly. From an initial $P_{\mathrm{NH} 3}$ of 0.15 mbar, the pressure was 0.01 mbar even after 10 minutes of purging. The GaN ALD is performed in a hot-wall reactor; therefore, a higher outgassing rate can be expected. However, the $P_{\mathrm{NH} 3}$ values are also 2-3 orders higher. Therefore, the residual $\mathrm{NH}_{3}$ during the purging presumably contributes to the anomalous GPC increase.

Moreover, surface reactions in the form of adduct to $-\mathrm{NH}_{2}-$ conversion can contribute to the GPC rise, even after $P_{\mathrm{NH} 3}$ has sufficiently reduced. Since the conversion first demands the formation of adjacent adduct units, and further, with the alternate arrangement of the $-\mathrm{CH}_{3}$ and $\mathrm{NH}_{3}$ groups of these units, satisfying these requirements, especially if the units are rotationally inhibited ${ }^{22}$, can make the conversion slow.
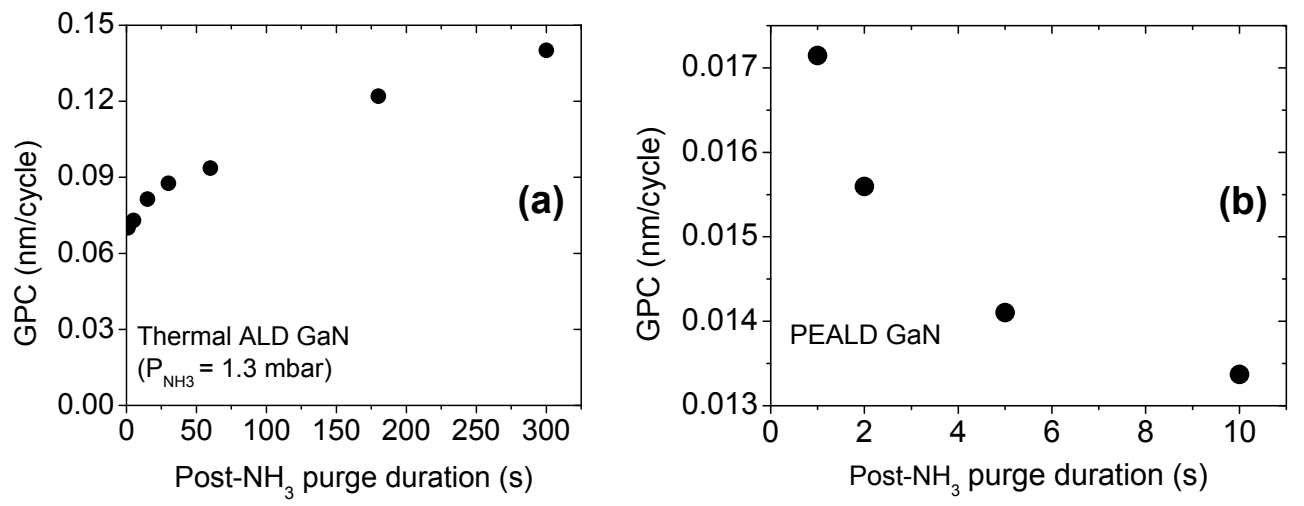

Fig. 5.7 (a) Anomalous increase of GPC with the purge duration following the $\mathrm{NH}_{3}$ pulse. The generic ALD recipe was: $0.1 \mathrm{~s} \mathrm{TMG} / 30 \mathrm{~s} \mathrm{Ar} / 30 \mathrm{~s} \mathrm{NH}_{3} / 5-300 \mathrm{~s} \mathrm{Ar}$, performed at $400{ }^{\circ} \mathrm{C}$ and $1.3 \mathrm{mbar}$ $P_{\mathrm{NH} 3}$. (b) The as-expected GPC behaviour obtained in GaN PEALD from TMG and $\mathrm{NH}_{3}$-plasma. (To note: the PEALD were performed in a separate reactor, using different gas settings. This result is for demonstration purpose only, and direct comparison between the two trends should not be made.)

\subsection{Growth analysis by in-situ SE}

The ALD mechanism was further studied by in-situ monitoring the GaN growth by SE, focussing on the shape of the growth curve. Since the deposition was performed by alternate precursor pulses and purges, growth occurred through clear well-defined steps (Fig. 5.8 a). The steps corresponded to the introduction of one or more precursors ${ }^{3}$, in this case TMG, presumably indicating a successful chemisorption of the latter (this is further discussed in Section 6.4). Fig. 5.8 b zooms into a single ALD cycle, showing the instants of 
the precursor pulses and purges. During the Ar purge following the TMG pulse, hardly any thickness variation is observed. Unlike the TMG pulse, the $\mathrm{NH}_{3}$ pulse does not cause a step, but rather leads to a gentle thickness decrease, that continues even during the subsequent Ar purge.

To note: the extremely small thickness variations within an ALD cycle (Fig. 5.8 b) are in fact the optical signal changes, as interpreted by the SE optical model. In addition to the actual thickness variations (we cannot exclude this), changes in the optical properties of the surface upon the corresponding chemical reactions may also cause this effect ${ }^{3}$. For example, the chemisorption of TMG and its nitridation into the $-\mathrm{NH}_{2}-$ links can cause the optical changes. The starting and the ending points of each cycle correspond to the real thickness values, providing the overall thickness increase per cycle, interpreted as the GPC. The film thickness (and therefore, GPC) are always verified by other ex-situ measurement techniques (e.g., SEM, TEM). A variation of thickness within a cycle, however, cannot be guaranteed, as explained above.

\subsubsection{Effect of $t_{\mathrm{NH} 3}$ on step height}

Fig. 5.8 c compares the shapes of individual ALD pulses obtained by SE for different $t_{\mathrm{NH} 3}$. The corresponding step-height increase is further quantified in Fig. 5.8 d. The gradual increase of the step-height presumably indicates an enhancement of TMG chemisorption with $t_{\mathrm{NH} 3}$. This can be explained by a higher surface coverage of $-\mathrm{NH}_{2}-$ linkages (recall Fig. 5.4 b) after a long $t_{\mathrm{NH} 3}$. These linkages serve as sites for TMG chemisorption in the subsequent cycle, increasing the surface coverage of the chemisorbed TMG, and resulting in a larger step. On the contrary, shorter $t_{\mathrm{NH} 3}$ leads to lower $-\mathrm{NH}_{2}-$ coverage. Therefore, parts of the surface still remain terminated by the $-\mathrm{CH}_{3}$ groups from previous TMG exposures. A weaker step is recorded as a result.

\subsubsection{Effect of $\boldsymbol{P}_{\mathrm{NH} 3}$ on step height}

The same mechanism comes into effect when $P_{\mathrm{NH} 3}$ is increased (Fig. 5.8 e, f). A higher $P_{\mathrm{NH} 3}$ causes more nitridation of the TMG-chemisorbed surface, leading to a higher coverage of $-\mathrm{NH}_{2}-$ linkages. The TMG chemisorption consequently has high surface coverage, leading to larger steps. 

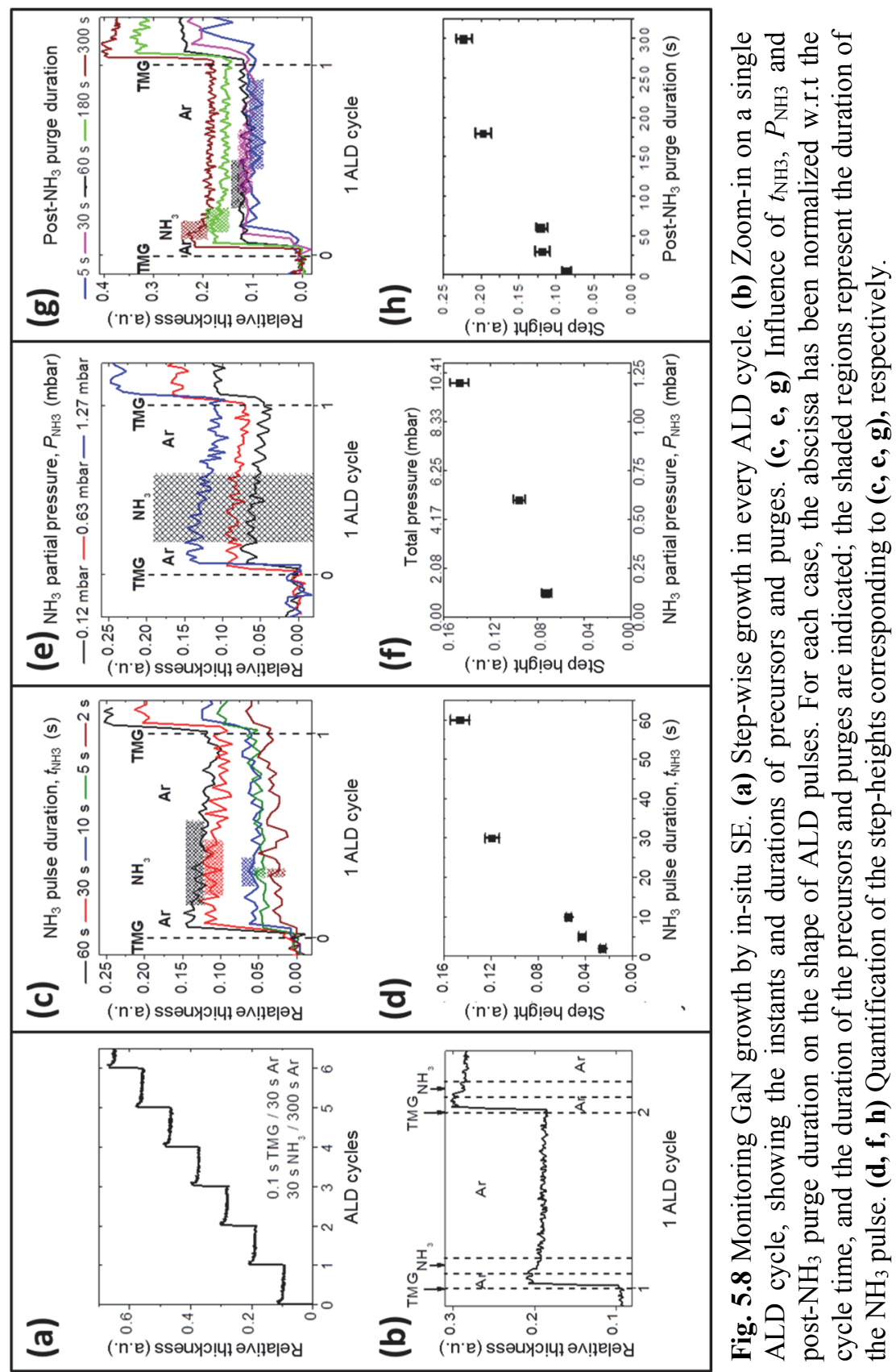


\subsubsection{Effect of post- $\mathrm{NH}_{3}$ purge duration on step height}

The dependence of GPC on the post- $\mathrm{NH}_{3}$ purge duration is reflected in the increasing step heights (Fig. 5.8 g). Longer purges increase the $-\mathrm{NH}_{2}-$ coverage (Section 5.6.2), creating sites for the subsequent $\mathrm{TMG}$ chemisorption. Fig. $\mathbf{5 . 8} \mathbf{h}$ shows a saturating trend of the step height at very long purges $(180 \mathrm{~s}, 300 \mathrm{~s})$. This implies that either the formation of $-\mathrm{NH}_{2}$ - approaches completion, or $P_{\mathrm{NH} 3}$ becomes too low to cause additional $-\mathrm{NH}_{2}-$ formation. The former implies the progression towards a monolayer of $\mathrm{GaN}$, especially because the step heights at $180 \mathrm{~s}$ and $300 \mathrm{~s}$ are greater than at maximum $t_{\mathrm{NH} 3}$ or $P_{\mathrm{NH} 3}($ Fig. $5.8 \mathbf{d}, \mathbf{f})$.

\subsection{Layer characterization}

\subsubsection{HR-SEM analysis}

Fig. 5.9 a shows a HR-ESB image of GaN layers deposited in Si trenches, for testing the conformality of the ALD recipe (see figure caption). Fig. 5.9 b is a HR-SEM image showing the trench-fill performance of the process.
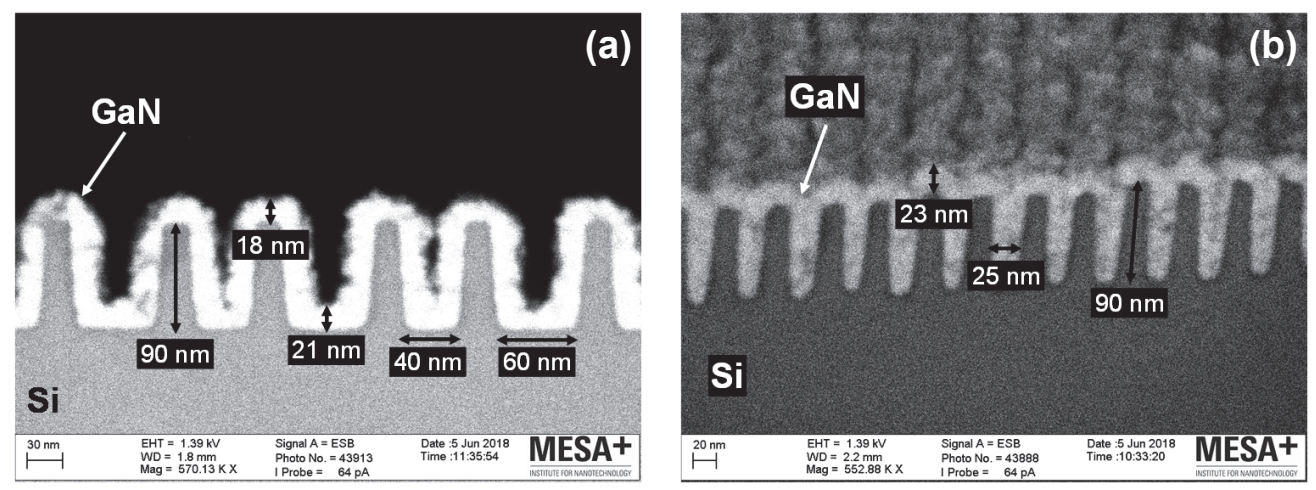

Fig. 5.9 (a) HR-ESB and (b) HR-SEM images of GaN layers showing their decent conformality and trench-filling capability, respectively. The layer was deposited with the ALD recipe: $0.1 \mathrm{~s}$ TMG / $30 \mathrm{~s}$ $\mathrm{Ar} / 30 \mathrm{~s} \mathrm{NH}_{3} / 60 \mathrm{~s} \mathrm{Ar}$, at $400{ }^{\circ} \mathrm{C}$ and $1.3 \mathrm{mbar} P_{\mathrm{NH} 3}$.

\subsubsection{GIXRD and HR-TEM analyses}

The formation of polycrystalline GaN was confirmed from GIXRD, acquired at an incident angle $(\omega)$ of $1^{\circ}$ (Fig. 5.10, bottom). The peaks correspond to hexagonal (wurtzitic) $\mathrm{GaN}$, as indicated by the blue bars (COD No. 9011658). The $d$-spacing of (100), (002) and 
(101) crystal planes are estimated to be $2.8 \AA, 2.6 \AA$ and $2.5 \AA$, respectively, which agrees with the database.

The polycrystalline structure is observed from HR-TEM (Fig. 5.10, top), which reveals crystalline domains oriented in different directions. The domain sizes (indicated by double-head arrows) are sub-10 nm. Fast Fourier Transform (FFT) analysis at several domains (one such domain is enclosed by the yellow box, and its FFT is shown in the inset) reveals $d$-spacing of $2.60 \pm 0.02 \AA$. This conforms to the (002) wurtzitic GaN crystal planes.

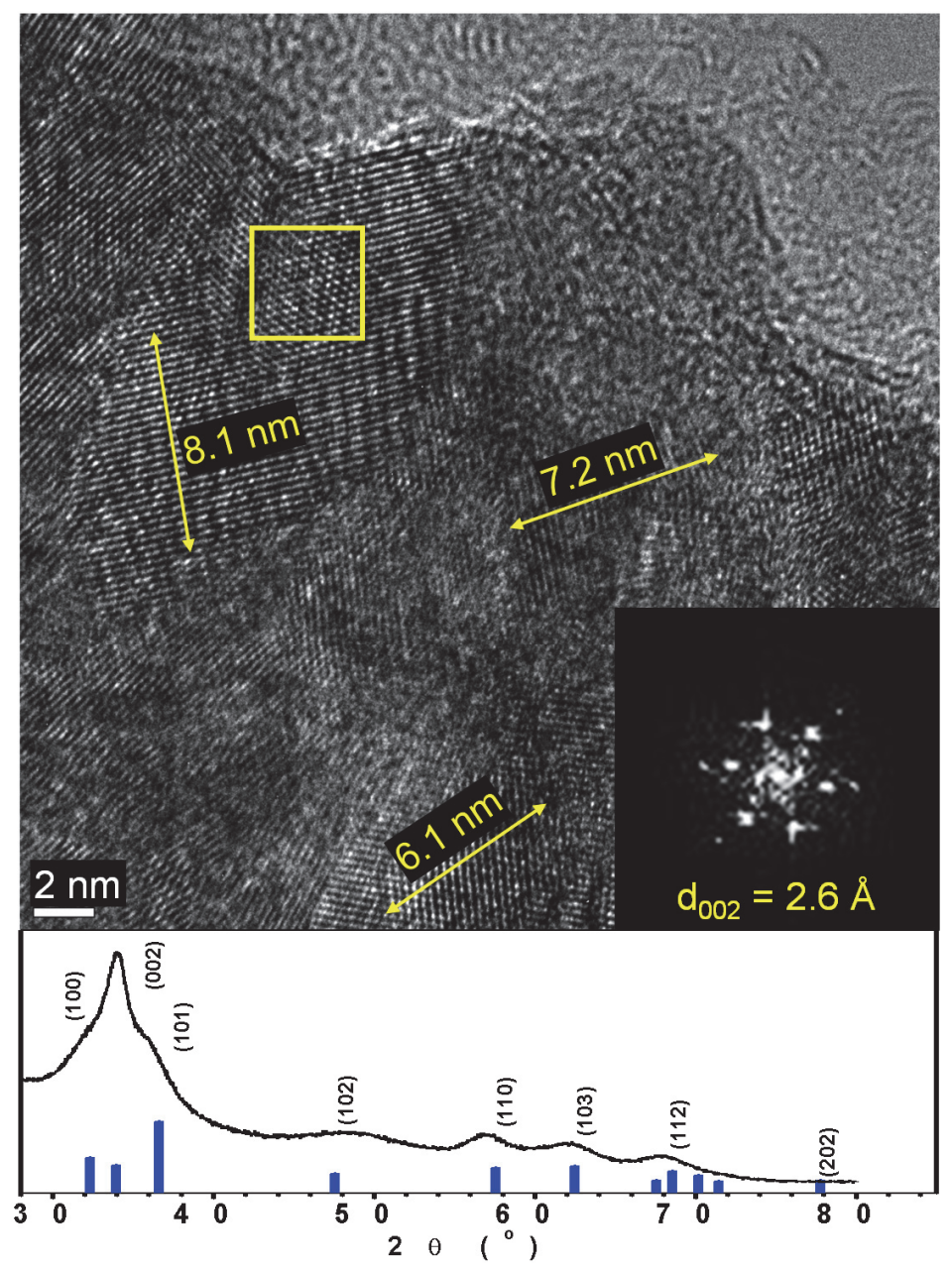

Fig. 5.10 (Bottom) GIXRD scan revealing the polycrystalline wurtzitic structure of the layer. The blue bars represent the peak positions for wurtzitic GaN. The parentheses denote their respective Miller indices. (Top) HR-TEM image showing the polycrystalline structure, with sub-10 nm domains. Inset: FFT analysis of a domain (in yellow box) revealing a $d$-spacing of $2.6 \AA$, consistent with wurtzitic (002) GaN crystal planes. 


\subsubsection{FTIR analysis}

Fig. 5.11 shows the FTIR transmission spectrum of the GaN layer. The spectrum is the ratio of the single beam transmittance spectra of the layer and that of a reference $\mathrm{Si}$ wafer. The principle absorption at $534 \mathrm{~cm}^{-1}$ corresponds to the $\mathrm{Ga}-\mathrm{N}$ stretching mode, which is in good agreement with the same for wurtzitic GaN films reported at $533 \mathrm{~cm}^{-1(52)}$. The absorptions due to the $-\mathrm{NH}_{2}$ bending and stretching modes were also identified at $945 \mathrm{~cm}^{-1}$ and $3250 \mathrm{~cm}^{-1}$, respectively ${ }^{53}$. These absorptions suggest that the growth mechanism could indeed have involved the $-\mathrm{NH}_{2}-$ linkages, and that, some of these linkages did not react with TMG. The evidence of some Ga-H bonds is observed from the absorption at $704 \mathrm{~cm}^{-1}$, corresponding to $\mathrm{Ga}-\mathrm{H}$ bending vibration ${ }^{53}$. The absorption at $1100 \mathrm{~cm}^{-1}$ corresponds to Si-O bonds, which originate from the native oxide on the wafer. The overshoot between 2000 and $2500 \mathrm{~cm}^{-1}$ originates from small variations in $\mathrm{CO}_{2}$ level in the spectrometer between the measurements.

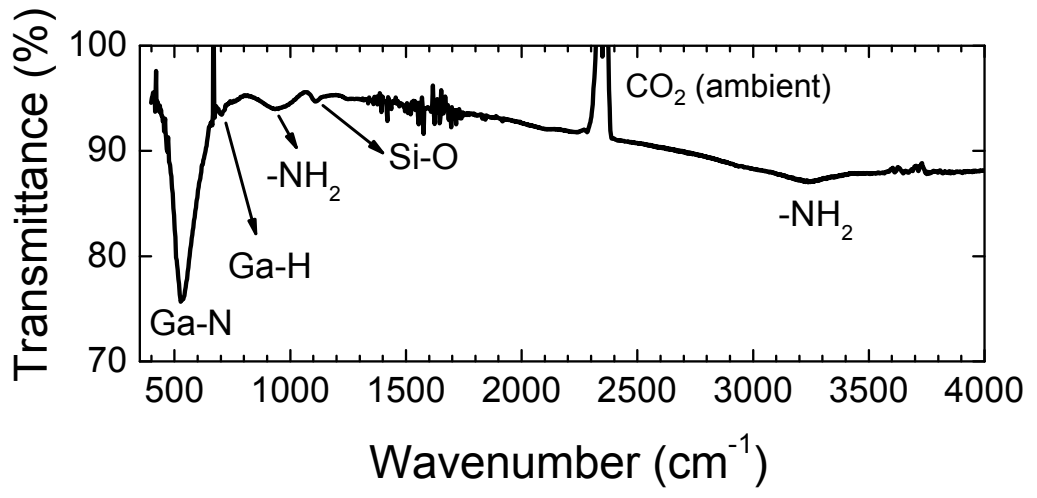

Fig. 5.11 FTIR transmission spectrum of the GaN layer showing the various chemical bonds.

\subsubsection{XPS analysis}

Fig. 5.12 a shows the depth profiled composition of a $14 \mathrm{~nm}$ thick layer on Si. XPS reveals that the layer is not stoichiometric, but rather rich in Ga. A low level of oxygen and hardly any carbon is detected. The average composition is estimated as $\mathrm{Ga}_{0.53} \mathrm{~N}_{0.39} \mathrm{C}_{0.01} \mathrm{O}_{0.07}$.

To understand the origin of the non-stoichiometric composition, the chemical bonding inside the layer was analysed, focussing on the photoelectron Ga $2 \mathrm{p}_{3 / 2}$ spectrum (Fig. 5.12 b) and the photoelectron $\mathrm{N} 1 \mathrm{~s}$ - Auger $\mathrm{Ga}_{2} \mathrm{M}_{45} \mathrm{M}_{45}$ complex (Fig. 5.12 c). Deconvolution of the complex yielded the N 1s band at $397.1 \mathrm{eV} \mathrm{BE}$ (in blue) and the Auger triplet at a lower BE (in grey). The $\mathrm{N}$ 1s BE agrees well with the literature-reported BE of $\mathrm{N}-\mathrm{Ga}$ bonds $(396.9 \mathrm{eV})$ in poly-GaN films ${ }^{7}$. 
Whereas the Ga $2 \mathrm{p}_{3 / 2}$ spectrum of a perfectly-stoichiometric GaN layer should allow fitting only by one Gaussian-Lorentzian band (i.e., signifying a single bonding environment: Ga-N), the spectrum of Fig. 5.12 b is best deconvoluted by two bands, suggesting at least two bonding environments of $\mathrm{Ga}$ in the layer. The major band (at $1117.8 \mathrm{eV}$, occupying $83 \%$ of the peak area) corresponds to the $\mathrm{Ga}-\mathrm{N}$ bond due to its excellent match with the reported $\mathrm{BE}$ of $1117.8 \mathrm{eV}^{54}$ or $1117.9 \mathrm{eV}^{55}$. The minor band (at $1117.3 \mathrm{eV}$, occupying the remaining area) implies additional bonding environments of $\mathrm{Ga}$.

From the depth profiled XPS composition and the FTIR spectrum, the relevant candidates for the minor band are $\mathrm{Ga}-\mathrm{Ga}, \mathrm{Ga}-\mathrm{O}$ and $\mathrm{Ga}-\mathrm{H}$ bonds. Based on the electronegativity differences between $\mathrm{Ga}, \mathrm{H}, \mathrm{N}$ and $\mathrm{O}(1.81,2.20,3.04$ and 3.44, respectively ${ }^{47}$ ), the $\mathrm{Ga}-\mathrm{O}$ bond is expected at a higher $\mathrm{BE}$ than the $\mathrm{Ga}-\mathrm{N}$ bond ${ }^{56}$. Indeed, it has been reported at $1.3 \mathrm{eV}$ higher $\mathrm{BE}$ than $\mathrm{Ga}-\mathrm{N}^{57}$. However, since the O-content in our films is low, the band is submerged under the strong $\mathrm{Ga}-\mathrm{N}$ band.

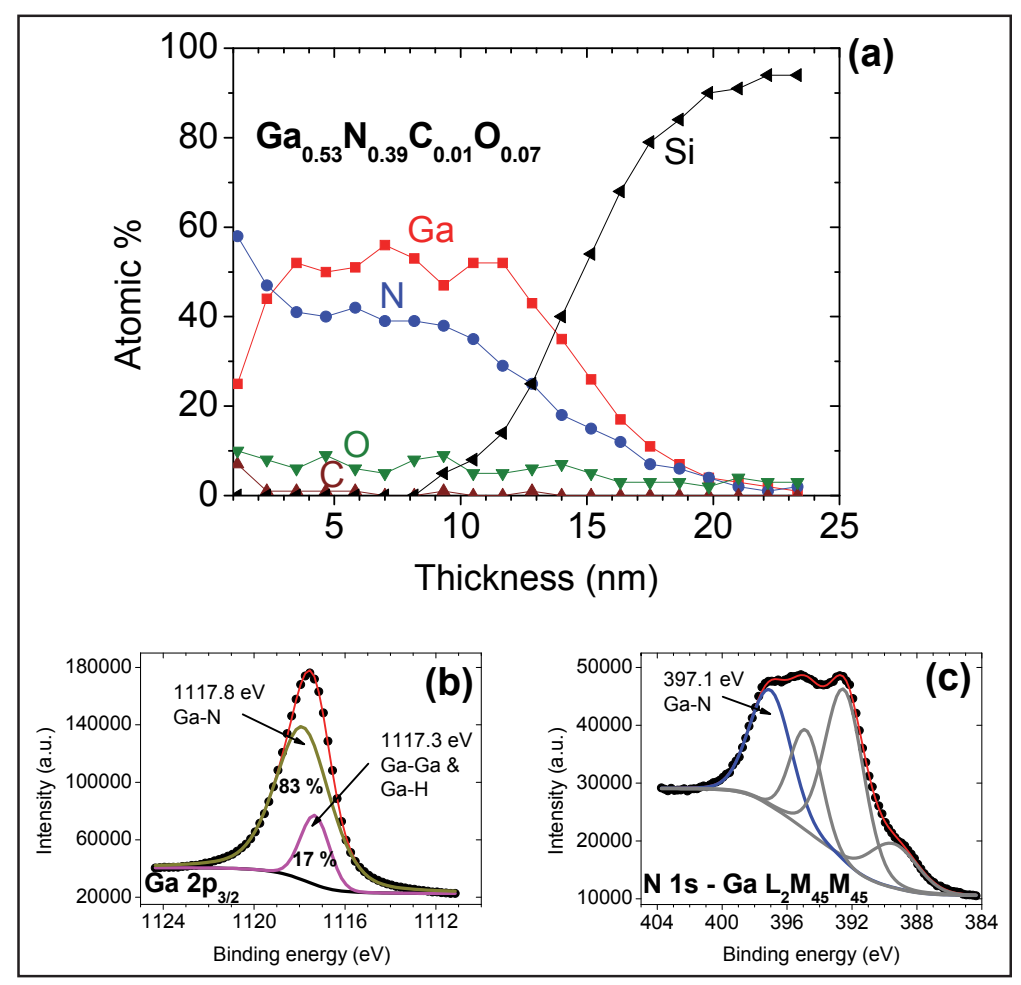

Fig. 5.12 (a) Sputter depth profiled XPS composition of the layer, deposited on Si, with the ALD recipe: $0.1 \mathrm{~s} \mathrm{TMG} / 30 \mathrm{~s} \mathrm{Ar} / 30 \mathrm{~s} \mathrm{NH} / 60 \mathrm{~s} \mathrm{Ar}$, at $400{ }^{\circ} \mathrm{C}$ and $1.3 \mathrm{mbar} P_{\mathrm{NH} 3}$. The average composition is indicated in the figure. (b) Photoelectron Ga 2 $\mathrm{p}_{3 / 2}$ and (c) Photoelectron N 1s - Auger $\mathrm{Ga} \mathrm{L}_{2} \mathrm{M}_{45} \mathrm{M}_{45}$ spectra from the layer bulk. These spectra have been deconvoluted to reveal the various bonding environment. 
The remaining candidates for the minor band are $\mathrm{Ga}-\mathrm{Ga}$ and $\mathrm{Ga}-\mathrm{H}$ bonds. The $\mathrm{BE}$ of the former is reported at $1116.5 \mathrm{eV}^{58}$, while the latter is reported around $1 \mathrm{eV}$ lower than the $\mathrm{Ga}-\mathrm{N} \mathrm{BE}{ }^{59}$, therefore around $1116.8 \mathrm{eV}$ in our layer. The minor band, peaked at $1117.3 \mathrm{eV}$, may be ascribed to be contributed by these two bonds. Although at a very similar $\mathrm{BE}$, the possibility of the $\mathrm{Ga}-\mathrm{C}$ bond (at $1117.2 \mathrm{eV}^{26}$ ) is ignored due to the ultra-low $\mathrm{C}$-content in the layer (Fig. 5.12 a). Finer deconvolution between $\mathrm{Ga}-\mathrm{Ga}$ and $\mathrm{Ga}-\mathrm{H}$ was not attempted because the measurement was limited by the instrumental resolution of $0.4 \mathrm{eV}$.

5.8.4.1 Stoichiometry control of the layers: The obvious question is: why is the layer Garich even though it was supposedly grown in a self-limiting fashion? The known phenomenon of the preferential sputtering of $\mathrm{N}$ over Ga during depth profiling of $\mathrm{GaN}^{54}$ may not be the cause, since that would cause only minor ( $2-3$ at. \%) excess of $\mathrm{Ga}^{17}$. It is more likely that during the growth, not all TMG-chemisorbed sites had converted into GaN; i.e., either the adduct formation had not occurred, or the adduct was not converted into the $-\mathrm{NH}_{2}-$ linkages. The simultaneous thermal dissociation of the TMG also cannot be ignored at the current deposition temperature $\left(400{ }^{\circ} \mathrm{C}\right)$, resulting in partial/complete $-\mathrm{CH}_{3}$ detachment ${ }^{26}$. The dissociated species might not be expected to participate in the adduct formation owing to their lack of $-\mathrm{CH}_{3}$ ligands. They would instead accumulate as $\mathrm{Ga}$ clusters, causing the observed Ga-richness of the layers. Increasing the deposition temperature enhances the formation of these clusters ${ }^{17}$. The longer (purge, pulse) duration may additionally enhance the Ga-richness, since the (presumably slow) thermal dissociation of the chemisorbed TMG is an ongoing process.

The means to more efficiently nitridize the chemisorbed TMG is to further increase the $P_{\mathrm{NH} 3}$ or $t_{\mathrm{NH} 3}$. However, the former can be a limitation in many reactor designs and can also introduce contaminants like moisture. The latter can (i) pose impractically long cycle times, and (ii) increase the Ga-richness. In practice, using short cycle times (especially reducing the purge times), even at the risk of venturing outside true ALD conditions, allows to minimize the extent of TMG dissociation at every cycle. Indeed, shortening the purge duration from 30 and $60 \mathrm{~s}$ to only $4 \mathrm{~s}$ after each precursor, resulted in a nearly-stoichiometric composition of $\mathrm{Ga}_{0.46} \mathrm{~N}_{0.44} \mathrm{C}_{0.02} \mathrm{O}_{0.08}{ }^{1}$. 


\subsection{Conclusions}

This chapter showed that low temperature thermal ALD of polycrystalline GaN from $\mathrm{TMG}$ and $\mathrm{NH}_{3}$ precursors is indeed possible without cracking the $\mathrm{NH}_{3}$ into radicals. Carbon-free $\mathrm{GaN}$ layers were grown between $375-425^{\circ} \mathrm{C}$, with a GPC as high as $0.1 \mathrm{~nm} /$ cycle.

In order to achieve this, based on the pre-reported role of the TMA: $\mathrm{NH}_{3}$ surface adduct in AIN ALD, and the role of the TMG: $\mathrm{NH}_{3}$ gas phase adduct in GaN MOCVD, we hypothesized the existence of an analogous $\mathrm{TMG}: \mathrm{NH}_{3}$ surface adduct which facilitates the GaN ALD. The proposed ALD model involved (i) the reversible formation of the TMG:NH 3 surface adduct upon the physisorption of $\mathrm{NH}_{3}$ on a TMG-chemisorbed surface site, and (ii) the interaction between the $-\mathrm{CH}_{3}$ and $\mathrm{NH}_{3}$ groups of neighbouring adduct units, leading to the irreversible formation of a $\mathrm{Ga}-\mathrm{NH}_{2}-\mathrm{Ga}$ linkage. The proposed ALD mechanism could also be viewed as the replication of the adduct pathway, prevalent in GaN MOCVD, into equivalent surface reactions.

The kinetics of the reversible surface adduct formation and its irreversible conversion into $-\mathrm{NH}_{2}$ - linkage were simulated using Mathcad. The results showed that whereas the surface coverage of the adduct was primarily dependent on the $\mathrm{NH}_{3}$ partial pressure, the linkage formation was strongly dependent on the $\mathrm{NH}_{3}$ pulse duration. The experimental observation of the strong GPC dependence on both these parameters supported the simulations. The pressure-dependence was indeed the novelty of the ALD process - the key to grow GaN thermally at low temperatures.

The stepwise growth of the layer, as monitored by in-situ SE, supplemented the ALD model. Increasing $t_{\mathrm{NH} 3}, P_{\mathrm{NH} 3}$ and the post- $\mathrm{NH}_{3}$ purge duration all resulted in stronger step heights following the TMG pulse; thus implying a higher surface coverage of the $-\mathrm{NH}_{2}-$ , and hence chemisorbed TMG, from all these changes.

Ex-situ layer characterization revealed (i) excellent conformality and trench-filling performance of the ALD recipe from SEM images, (ii) polycrystalline wurtzitic structure with sub-10 nm grains from GIXRD and TEM, (iii) evidence of residual $-\mathrm{NH}_{2}$ bonds from FTIR spectroscopy, and (iv) an average Ga-rich stoichiometry of $\mathrm{Ga}_{0.53} \mathrm{~N}_{0.39} \mathrm{C}_{0.01} \mathrm{O}_{0.07}$, with the detection of Ga-Ga chemical bonds with XPS. The reason behind the excess Ga was attributed to the (continuing) partial dissociation of the chemisorbed TMG at the ALD temperature of $400{ }^{\circ} \mathrm{C}$, i.e., without converting into the $-\mathrm{NH}_{2}-$ linkages. Lack of a complete nitridation and the long purge times were the possible reasons. Shortening the long cycle times (at the risk of processing outside the ALD window) resulted in a nearly-stoichiometric composition of $\mathrm{Ga}_{0.46} \mathrm{~N}_{0.44} \mathrm{C}_{0.02} \mathrm{O}_{0.08}$. The negligible $\mathrm{C}$-content in both cases proved that $\mathrm{a}$ 
radical-assisted route was not necessary to extract the $-\mathrm{CH}_{3}$ groups of TMG to form $\mathrm{GaN}$; the hypothesized surface adduct pathway efficiently achieved this.

The question remains: was the described deposition a true ALD process? The selflimiting manner of the adduct to $-\mathrm{NH}_{2}$ - linkage conversion does imply an ALD process. However, the absence of a saturation regime between GPC and $t_{\mathrm{NH} 3}$ implies otherwise. The demonstrated pressure dependence of the growth kinetics is also atypical for ALD. Finally, in the absence of a dedicated surface study, this work can neither prove the existence of the surface adduct nor validate the proposed ALD model. However, both can adequately explain the experimental observations. 


\section{References}

1. S. Banerjee and A. Y. Kovalgin, ECS Transactions, 2018, 86, 21-29.

2. H. Profijt, S. Potts, M. Van de Sanden and W. Kessels, Journal of Vacuum Science \& Technology A: Vacuum, Surfaces, and Films, 2011, 29, 050801.

3. A. Y. Kovalgin, M. Yang, S. Banerjee, R. O. Apaydin, A. A. I. Aarnink, S. Kinge and R. A. M. Wolters, Advanced Materials Interfaces, 2017, 4, 1700058.

4. G. Gupta, S. Banerjee, S. Dutta, A. A. I. Aarnink, J. Schmitz, A. Y. Kovalgin and R. J. E. Hueting, Journal of Applied Physics, 2018, 124, 084503.

5. M. Alevli and N. Gungor, Journal of Vacuum Science \& Technology A: Vacuum, Surfaces, and Films, 2018, 36, 01A110.

6. C. Ozgit, I. Donmez, M. Alevli and N. Biyikli, Journal of Vacuum Science \& Technology A: Vacuum, Surfaces, and Films, 2012, 30, 01A124.

7. C. Ozgit-Akgun, E. Goldenberg, A. K. Okyay and N. Biyikli, Journal of Materials Chemistry C, 2014, 2, 2123-2136.

8. S. Kizir, A. Haider and N. Biyikli, Journal of Vacuum Science \& Technology A: Vacuum, Surfaces, and Films, 2016, 34, 041511.

9. P. Motamedi, N. Dalili and K. Cadien, Journal of Materials Chemistry C, 2015, 3, 74287436.

10. P. Pansila, K. Kanomata, M. Miura, B. Ahmmad, S. Kubota and F. Hirose, Applied Surface Science, 2015, 357, 1920-1927.

11. J. K. Sprenger, A. S. Cavanagh, H. Sun, K. J. Wahl, A. Roshko and S. M. George, Chemistry of Materials, 2016, 28, 5282-5294.

12. O. H. Kim, D. Kim and T. Anderson, Journal of Vacuum Science \& Technology A: Vacuum, Surfaces, and Films, 2009, 27, 923-928.

13. H. Tsuchiya, M. Akamatsu, M. Ishida and F. Hasegawa, Japanese Journal of Applied Physics, 1996, 35, L748.

14. A. Koukitu, Y. Kumagai, T. Taki and H. Seki, Japanese Journal of Applied Physics, 1999, 38, 4980 .

15. M. Mesrine, N. Grandjean and J. Massies, Applied Physics Letters, 1998, 72, 350-352.

16. M. Alevli, A. Haider, S. Kizir, S. A. Leghari and N. Biyikli, Journal of Vacuum Science \& Technology A: Vacuum, Surfaces, and Films, 2016, 34, 01A137.

17. S. Banerjee, A. J. Onnink, S. Dutta, A. A. I. Aarnink, D. J. Gravesteijn and A. Y. Kovalgin, The Journal of Physical Chemistry C, 2018, 122, 29567-29576. 
18. J. Sumakeris, Z. Sitar, K. Ailey-Trent, K. More and R. Davis, Thin Solid Films, 1993, 225, 244-249.

19. H. Wang, S. Huang, T. Yan, J. Gong, T. Lin and Y. Chen, Materials Science and Engineering: B, 1999, 57, 218-223.

20. N. Karam, T. Parodos, P. Colter, D. McNulty, W. Rowland, J. Schetzina, N. El-Masry and S. M. Bedair, Applied Physics Letters, 1995, 67, 94-96.

21. M. Bartram, T. Michalske and J. Rogers Jr, The Journal of Physical Chemistry, 1991, 95, 4453-4463.

22. M. Bartram, T. Michalske, J. Rogers Jr and R. Paine, Chemistry of Materials, 1993, 5, 14241430 .

23. F. C. Sauls and L. V. Interrante, Coordination Chemistry Reviews, 1993, 128, 193-207.

24. R. Puurunen, A. Root, P. Sarv, S. Haukka, E. Iiskola, M. Lindblad and A. Krause, Applied Surface Science, 2000, 165, 193-202.

25. M. J. Bronikowski and R. J. Hamers, Surface Science, 1996, 348, 311-324.

26. F. Lee, A. Backman, R. Lin, T. Gow and R. Masel, Surface Science, 1989, 216, 173-188.

27. D. Sengupta, S. Mazumder, W. Kuykendall and S. A. Lowry, Journal of Crystal Growth, 2005, 279, 369-382.

28. D. Mazzarese, A. Tripathi, W. Conner, K. Jones, L. Calderon and D. Eckart, Journal of Electronic Materials, 1989, 18, 369-377.

29. T. F. Kuech, in Handbook of Crystal Growth: Thin Films and Epitaxy (Second Edition), Elsevier, 2015, pp. 869-907.

30. T. Mihopoulos, Reaction and Transport Processes in OMCVD: Selective and Group IIINitride Growth, Ph.D Thesis, Massachusetts Institute of Technology, 1999.

31. R. P. Parikh and R. A. Adomaitis, Journal of Crystal Growth, 2006, 286, 259-278.

32. Q. An, A. Jaramillo-Botero, W.-G. Liu and W. A. Goddard III, The Journal of Physical Chemistry C, 2015, 119, 4095-4103.

33. K. Wang, J. Singh and D. Pavlidis, Journal of Applied Physics, 1994, 76, 3502-3510.

34. M. J. Almond, C. E. Jenkins, D. A. Rice and K. Hagen, Journal of Organometallic Chemistry, 1992, 439, 251-261.

35. A. Thon and T. F. Kuech, Applied Physics Letters, 1996, 69, 55-57.

36. J. R. Creighton and G. T. Wang, The Journal of Physical Chemistry A, 2005, 109, 1055410562. 
37. G. T. Wang and J. R. Creighton, The Journal of Physical Chemistry A, 2006, 110, 10941099.

38. J. R. Creighton, G. T. Wang, W. G. Breiland and M. E. Coltrin, Journal of Crystal Growth, 2004, 261, 204-213.

39. U. Bergmann, V. Reimer and B. Atakan, Physical Chemistry Chemical Physics, 1999, 1, 5593-5599.

40. B. Mondal, D. Mandal, D. Ghosh and A. K. Das, The Journal of Physical Chemistry A, 2010, 114, 5016-5025.

41. A. Y. Timoshkin and H. F. Schaefer Iii, The Journal of Physical Chemistry C, 2008, 112, 13816-13836.

42. D. Sengupta, The Journal of Physical Chemistry B, 2003, 107, 291-297.

43. S. Ravasio, T. Momose, K. Fujii, Y. Shimogaki, M. Sugiyama and C. Cavallotti, The Journal of Physical Chemistry A, 2015, 119, 7858-7871.

44. A. Pelekh and R. W. Carr, The Journal of Physical Chemistry A, 2001, 105, 4697-4701.

45. A. Tachibana, O. Makino, S. Tanimura, H. Tokunaga, N. Akutsu and K. Matsumoto, Physica Status Solidi (A), 1999, 176, 699-703.

46. J. R. Creighton and G. T. Wang, The Journal of Physical Chemistry A, 2005, 109, 133-137.

47. A. Allred, Journal of Inorganic and Nuclear Chemistry, 1961, 17, 215-221.

48. J. R. Creighton, G. T. Wang and M. E. Coltrin, Journal of Crystal Growth, 2007, 298, 2-7.

49. R. I. Masel, Principles of Adsorption and Reaction on Solid Surfaces, John Wiley \& Sons, 1996.

50. A. de Castro, D. Alegre and F. Tabarés, Nuclear Materials and Energy, 2016, 9, 1-5.

51. A. J. Onnink, R. O. Apaydin, J. Schmitz and A. Y. Kovalgin, EuroCVD 22-Baltic ALD 16 Conf., Luxembourg, June 2019.

52. T. Miyazaki, K. Takada, S. Adachi and K. Ohtsuka, Journal of Applied Physics, 2005, 97, 093516.

53. G. Socrates, Infrared and Raman Characteristic Group Frequencies: Tables and Charts, John Wiley \& Sons, 2001.

54. R. Carin, J. Deville and J. Werckmann, Surface and Interface Analysis, 1990, 16, 65-69.

55. Y. Jia, J. S. Wallace, E. Echeverria, J. A. Gardella Jr and U. Singisetti, Physica Status Solidi (B), 2017, 254, 1600681. 
56. N. Watkins, G. Wicks and Y. Gao, Applied Physics Letters, 1999, 75, 2602-2604.

57. S. Wolter, B. Luther, D. Waltemyer, C. Önneby, S. Mohney and R. Molnar, Applied Physics Letters, 1997, 70, 2156-2158.

58. J. L. Bourque, M. C. Biesinger and K. M. Baines, Dalton Transactions, 2016, 45, 7678-7696.

59. M.-Y. Kong, J.-P. Zhang, X.-L. Wang and D.-Z. Sun, Journal of Crystal Growth, 2001, 227, 371-375.

60. C. Theodoropoulos, T. Mountziaris, H. Moffat and J. Han, Journal of Crystal Growth, 2000, 217, 65-81. 


\section{Appendix 5: Mathcad script for simulating the TMG: $\mathrm{NH}_{3}$ surface adduct and GaN formation:}

\section{Modeling of GaN growth process}

$\begin{array}{lll}\mathrm{m}_{\mathrm{e}}:=9.1093897 \cdot 10^{-31} \cdot \mathrm{kg} & \text { Electron mass in vacuum } & \mathrm{mbar}:=10^{-3} \mathrm{bar} \\ \mathrm{k}_{\mathrm{b}}:=1.380658 \cdot 10^{-23} \cdot \frac{\mathrm{joule}}{\mathrm{K}} & \text { Boltzmann's constant } & \mathrm{nm}=1 \times 10^{-9} \mathrm{~m} \\ \mathrm{~N}_{\mathrm{A}}:=6.0221367 \cdot 10^{23} \cdot \mathrm{mole}^{-1} & \text { Avogadro constant } & \mathrm{eV}:=1.6 \cdot 10^{-19} \mathrm{~J} \\ \mathrm{~N}_{\mathrm{GaN}}:=1 \cdot 10^{22} \cdot \mathrm{cm}^{-3} & \text { Assumed Number of Ga-N pairs incorporated per unit volume into GaN } \\ \mathrm{N}_{\mathrm{S}}:=1 \cdot 10^{15} \cdot \mathrm{cm}^{-2} & \text { Assumed Number of Ga-N pairs incorporated per unit surface of GaN } \\ \mathrm{T}:=700 \cdot \mathrm{K} & \end{array}$

Input Deposition Parameters

$\mathrm{P}_{\text {tot }}:=14.75 \times 10^{-0} \mathrm{mbar}$

$\mathrm{Q}_{\mathrm{NH} 3}:=4 \mathrm{~cm}^{3} \cdot \mathrm{min}^{-1} \quad \mathrm{Q}_{\mathrm{Ar}}:=55 \mathrm{~cm}^{3} \cdot \mathrm{min}^{-1}$

$P_{A r}:=\frac{Q_{A r}}{Q_{A r}+Q_{N H 3}} \cdot P_{\text {tot }}$

$\mathrm{P}_{\mathrm{Ar}}=13.75 \cdot \mathrm{mbar}$

$\mathrm{C}_{\mathrm{Ar}}:=\frac{\mathrm{P}_{\mathrm{Ar}}}{\mathrm{k}_{\mathrm{b}} \cdot 300 \mathrm{~K}}$

$\mathrm{C}_{\mathrm{Ar}}=3.32 \times 10^{17} \cdot \mathrm{cm}^{-3}$

$P_{\mathrm{NH} 3}\left(P_{\text {tot }}\right):=\frac{Q_{\mathrm{NH}}}{\mathrm{Q}_{\mathrm{Ar}}+\mathrm{Q}_{\mathrm{NH} 3}} \cdot \mathrm{P}_{\text {tot }}$

$\mathrm{P}_{\mathrm{NH} 3}\left(\mathrm{P}_{\text {tot }}\right)=1 \cdot \mathrm{mbar}$

$\mathrm{C}_{\mathrm{NH} 3}\left(\mathrm{P}_{\text {tot }}\right):=\frac{\mathrm{P}_{\mathrm{NH} 3}\left(\mathrm{P}_{\text {tot }}\right)}{\mathrm{k}_{\mathrm{b}} \cdot 300 \mathrm{~K}}$

$\mathrm{C}_{\mathrm{NH} 3}\left(\mathrm{P}_{\text {tot }}\right)=2.414 \times 10^{16} \cdot \mathrm{cm}^{-3}$

$\mathrm{k}_{\text {ads }}:=1 \times 10^{-11} \cdot \mathrm{cm}^{3} \mathrm{~s}^{-1}$

$\mathrm{k}_{\mathrm{des}}:=1 \times 10^{6} \cdot \mathrm{s}^{-1}$

$\mathrm{K}:=\frac{\mathrm{k}_{\mathrm{ads}}}{\mathrm{k}_{\mathrm{des}}}$

$\mathrm{N}_{\text {TMG }}:=1 \cdot 10^{15} \cdot \mathrm{cm}^{-2}$

Surface coverage with TMG sites ready for the next step, i.e., adsorption of $\mathrm{NH} 3$ and forming the adduct.

Written by dr. A. Y. Kovalgin, University of Twente. 


\section{Langmuir kinetics - reversible reaction}

$$
\operatorname{CVRG}\left(t, P_{\text {tot }}\right):=\frac{\mathrm{K} \cdot \mathrm{C}_{\mathrm{NH} 3}\left(\mathrm{P}_{\text {tot }}\right)}{\left(\mathrm{K} \cdot \mathrm{C}_{\mathrm{NH} 3}\left(\mathrm{P}_{\mathrm{tot}}\right)+1\right)} \cdot\left[1-\exp \left[-\mathrm{k}_{\mathrm{des}} \cdot\left(\mathrm{K} \cdot \mathrm{C}_{\mathrm{NH} 3}\left(\mathrm{P}_{\mathrm{tot}}\right)+1\right) \cdot \mathrm{t} \cdot \mathrm{s}\right]\right] \frac{\mathrm{K} \cdot \mathrm{C}_{\mathrm{NH} 3}\left(\mathrm{P}_{\text {tot }}\right)}{\left(\mathrm{K} \cdot \mathrm{C}_{\mathrm{NH} 3}\left(\mathrm{P}_{\mathrm{tot}}\right)+1\right)}=0.194
$$

$\operatorname{CVRG}\left(t, P_{\text {tot }}\right)$ is the surface coverage of the available $N_{T M G}$ sites.

$$
\mathrm{t}:=0,0.0000001 . .0 .00001
$$

$0,0.0000001 . .0 .00001$

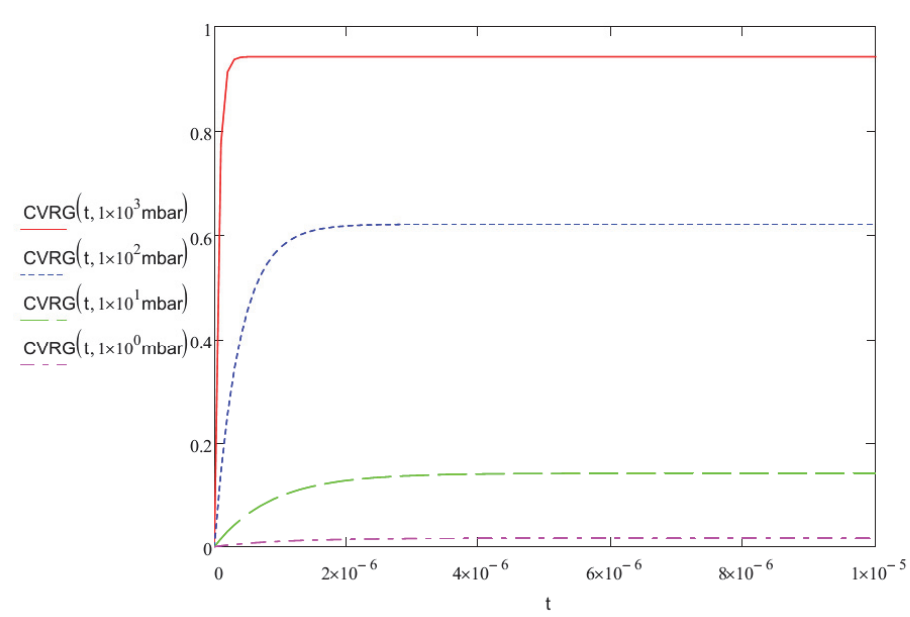

Next step: To add the second reaction between pre-formed adduct and neighbouring - $\mathrm{CH} 3$ group, thereby modeling the complete GaN growth. Due to a short time (i.e., the microsecond range) needed to reach equillibrium for the surface coverage, we can ignore time dependence for the first step. In other words, the total number of adduct species available for reaction 2 (i.e., adduct $+\mathrm{N}_{\text {TMG }}=\mathrm{GaN}$ ) will be:

$$
P_{\text {tot }}:=5 \times 10^{-0} \text { mbar } \quad \frac{\mathrm{K} \cdot \mathrm{C}_{\mathrm{NH} 3}\left(\mathrm{P}_{\text {tot }}\right)}{\left(\mathrm{K} \cdot \mathrm{C}_{\mathrm{NH} 3}\left(\mathrm{P}_{\text {tot }}\right)+1\right)} \cdot \mathrm{N}_{\mathrm{TMG}}=7.565 \times 10^{13} \cdot \mathrm{cm}^{-2}
$$

The second reaction can then be described by the following system of 3 equations: 
$\frac{\mathrm{d}(\mathrm{GaN})}{\mathrm{dt}}:=\mathrm{k}_{\mathrm{GaN}} \cdot$ (adduct) $\cdot \mathrm{N}_{\mathrm{TMG}}$

(adduct new $):=\left(\right.$ adduct $\left._{\text {old }}\right)-(\mathrm{GaN})$

$\left(\mathrm{N}_{\text {TMG.new }}\right):=\left(\mathrm{N}_{\text {TMG.old }}\right)-(\mathrm{GaN})$

$\mathrm{Nr}$. of the $\mathrm{NH} 2$-bridges (per $\mathrm{cm}^{-2}$ ) formed within one cycle as function of time:

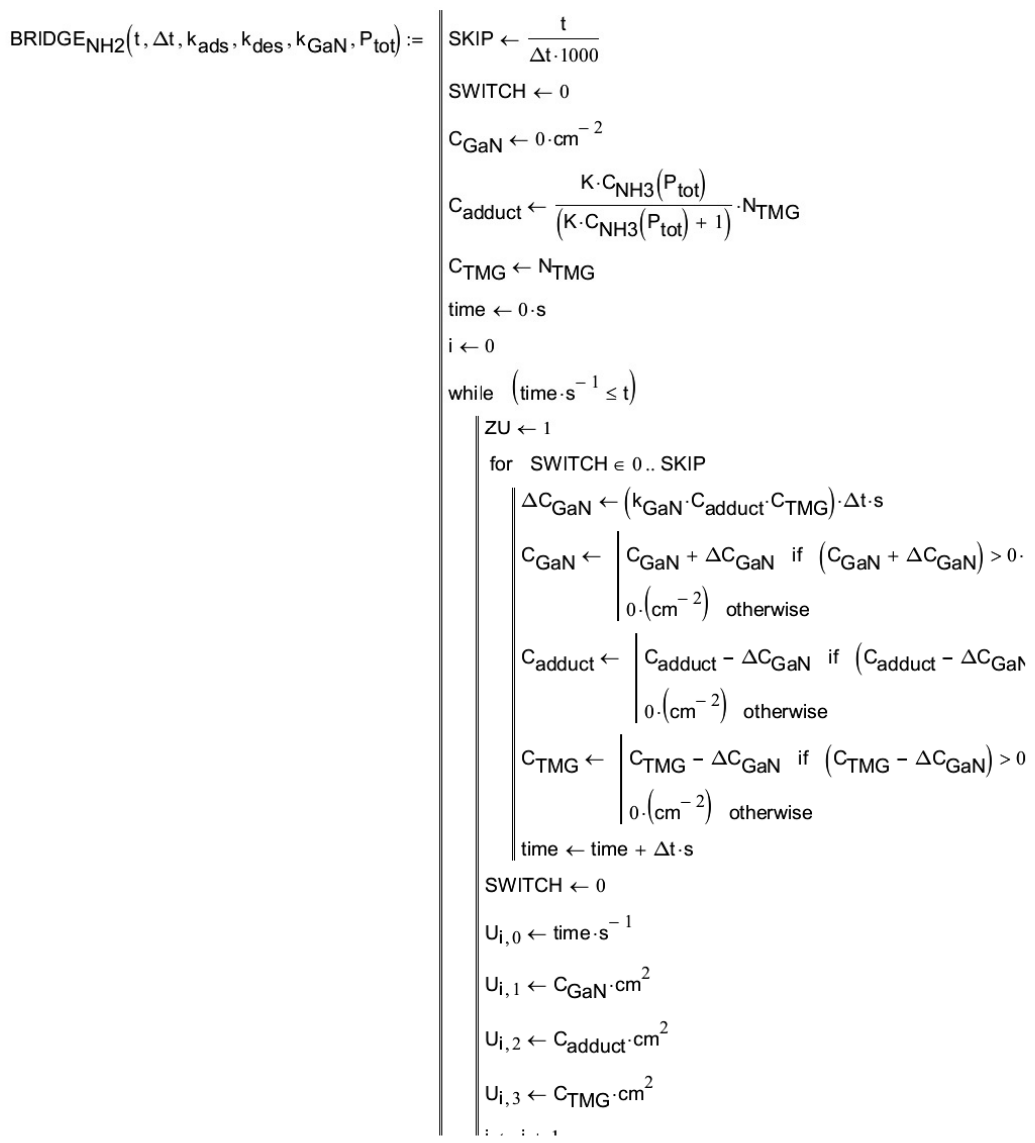




$$
\begin{aligned}
& \mathrm{t}:=100 \text { (seconds) } \quad \Delta \mathrm{t}:=10^{-3} \quad \| \mathrm{U}_{\text {(seconds) }}^{\| 1 \leftarrow \mathrm{t}+1} \quad \frac{\mathrm{t}}{\Delta \mathrm{t}}=1 \times 10^{5} \\
& k_{G a N}:=1 \times 10^{-16} \cdot \mathrm{cm}^{2} \mathrm{~s}^{-1} \quad k_{\text {ads }}=1 \times 10^{-11} \cdot \mathrm{cm}^{3} \mathrm{~s}^{-1} \quad k_{\text {des }}=1 \times 10^{6} \cdot \mathrm{s}^{-1}
\end{aligned}
$$

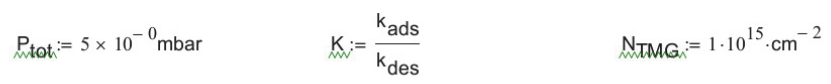

$$
\begin{aligned}
& \mathrm{C}_{\text {Surface }}:=\mathrm{BRIDGE}_{\mathrm{NH} 2}\left(\mathrm{t}, \Delta \mathrm{t}, \mathrm{k}_{\text {ads }}, \mathrm{k}_{\text {des }}, \mathrm{k}_{\mathrm{GaN}}, \mathrm{P}_{\text {tot }}\right) \\
& i:=0 . \text { rows }\left(C_{\text {Surface }}\right)-1 \\
& \mathrm{~T}_{\mathrm{i}}:=\mathrm{C}_{\text {Surface }_{\mathrm{i}, 0}} \quad \mathrm{C}_{\mathrm{GaN}_{\mathrm{i}}}:=\mathrm{C}_{\text {Surface }_{\mathrm{i}, 1}} \\
& \mathrm{C}_{\text {adduct }_{\mathrm{i}}}:=\mathrm{C}_{\text {Surface }_{\mathrm{i}, 2}} \quad \mathrm{C}_{\mathrm{TMG}_{\mathrm{i}}}:=\mathrm{C}_{\text {Surface }_{\mathrm{i}, 3}}
\end{aligned}
$$

Assuming that the formation of adduct is continious in time after each $\Delta$ t-step of reaction 2 . In other words, the remaining TMG sites can still act as adsorption sites due to their availability and promote adduct formation. In terms of the model this means that, after each $\Delta t$ step, the number of adduct species should be corrected w.r.t. the remaining $\mathrm{N}_{\mathrm{TMG}}$ and the surface coverage cov. 


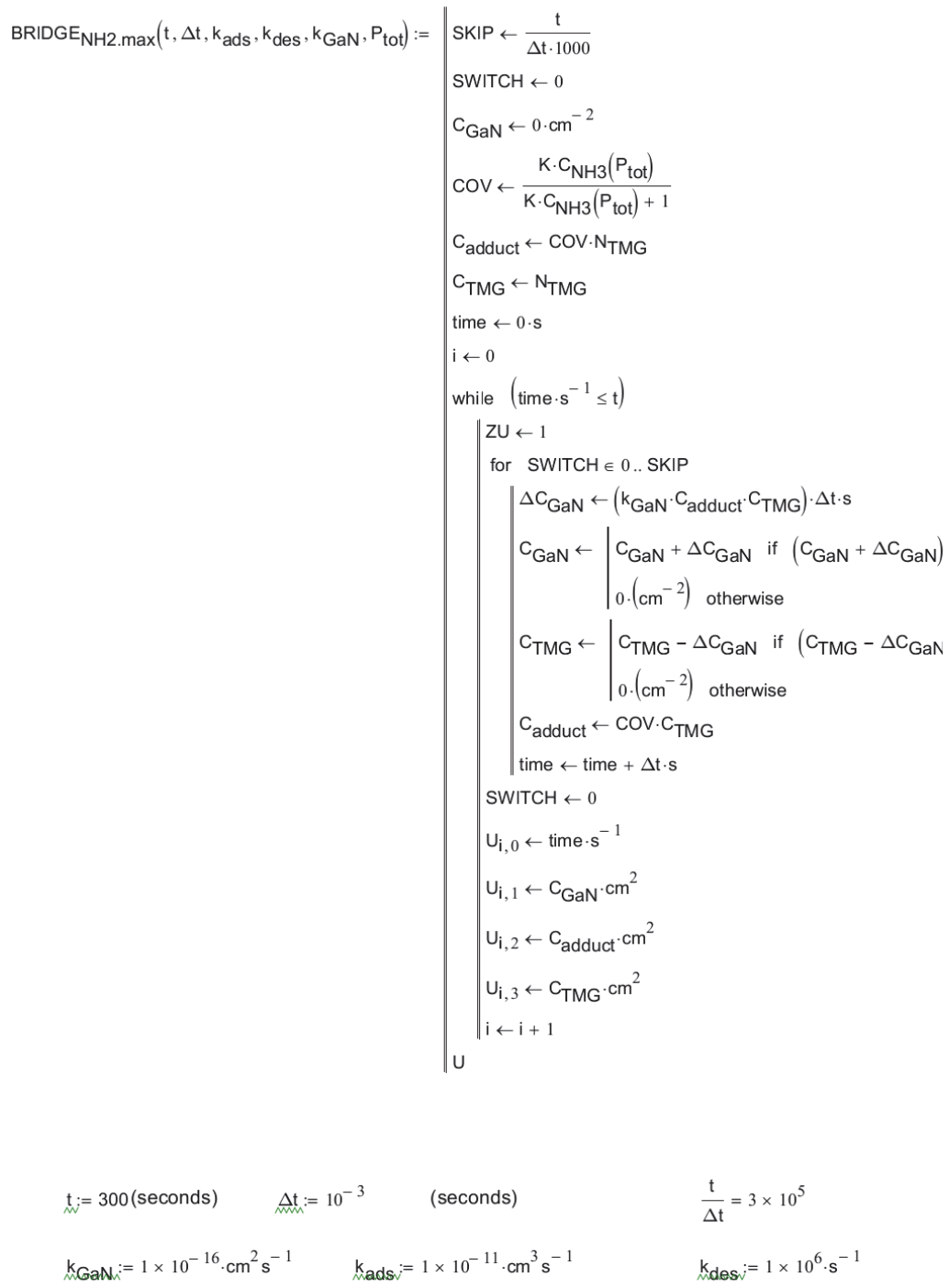



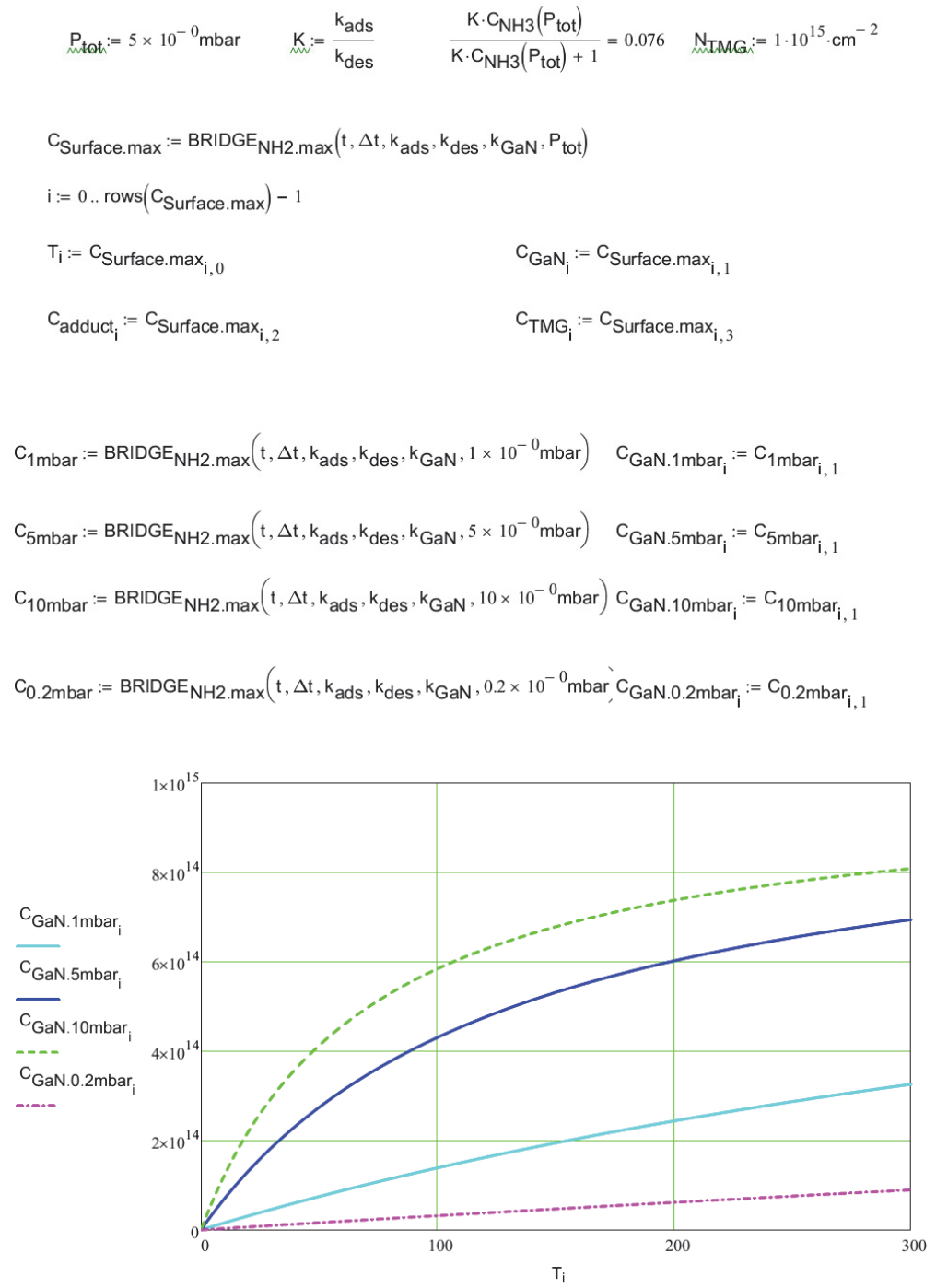
Assuming dependence of pressure on time, i.e., in fact that means modelling the long purge cycles with an exponential decrease of pressure. As is the previous example, the formation of adduct is continious in time after each $\Delta$ t-step of reaction 2.

$$
\begin{aligned}
& \text { PRESSURE }\left(\text { tim, } P_{\text {tot }}\right):=\frac{Q_{\mathrm{NH} 3}}{Q_{\mathrm{Ar}}+\mathrm{Q}_{\mathrm{NH} 3}} \cdot \mathrm{P}_{\text {tot }} \cdot \mathrm{mbar}^{-1} \cdot \exp \left(-5 \times 10^{-2} \cdot \mathrm{tim}\right) \\
& \operatorname{PRESSURE}\left(20, \mathrm{P}_{\text {tot }}\right)=0.125 \\
& \text { tim }:=0,1 \ldots 300 \\
& \operatorname{cov}\left(\mathrm{P}_{\text {tot }}\right):=\frac{\mathrm{K} \cdot \mathrm{C}_{\mathrm{NH} 3}\left(\mathrm{P}_{\text {tot }}\right)}{\mathrm{K} \cdot \mathrm{C}_{\mathrm{NH}}\left(\mathrm{P}_{\text {tot }}\right)+1} \quad \operatorname{Cov}\left(\mathrm{P}_{\text {tot }}\right)=0.076 \quad \mathrm{P}_{\text {tot }}=5 \cdot \mathrm{mbar} \quad \begin{array}{l}
\operatorname{Cov}\left(\mathrm{P}_{\text {tot }}\right) \text { is the max. } \\
\text { surface coverage }
\end{array} \\
& \text { BRIDGE }_{\text {NH2.purge }}\left(t, \Delta t, k_{\text {ads }}, k_{\text {des }}, k_{G a N}, P_{\text {tot }}\right):=\mid \operatorname{SKIP} \leftarrow \frac{t}{\Delta t \cdot 1000} \\
& \text { SWITCH } \leftarrow 0 \\
& \mathrm{C}_{\mathrm{GaN}} \leftarrow 2.3 \times 10^{14} \cdot \mathrm{cm}^{-2} \\
& \text { PRES } \leftarrow \mathrm{P}_{\text {tot }} \\
& \mathrm{C}_{\mathrm{TMG}} \leftarrow \mathrm{N}_{\mathrm{TMG}}-\mathrm{C}_{\mathrm{GaN}} \\
& \mathrm{C}_{\text {adduct }} \leftarrow \operatorname{COV} \text { (PRES) } \cdot \mathrm{C}_{\mathrm{TMG}} \\
& \text { time } \leftarrow 0 \cdot s \\
& \mathrm{i} \leftarrow 0 \\
& \text { while }\left(\text { time } \cdot \mathrm{s}^{-1} \leq \mathrm{t}\right) \\
& \mathrm{ZU} \leftarrow 1 \\
& \text { for SWITCH } \in 0 \text {.. SKIP } \\
& \Delta \mathrm{C}_{\mathrm{GaN}} \leftarrow\left(\mathrm{k}_{\mathrm{GaN}} \cdot \mathrm{C}_{\text {adduct }} \cdot \mathrm{C}_{\mathrm{TMG}}\right) \cdot \Delta \mathrm{t} \cdot \mathrm{s} \\
& \begin{array}{l||l}
\mathrm{C}_{\mathrm{GaN}} \leftarrow \| & \mathrm{C}_{\mathrm{GaN}}+\Delta \mathrm{C}_{\mathrm{GaN}} \text { if }\left(\mathrm{C}_{\mathrm{GaN}}+\Delta \mathrm{C}_{\mathrm{GaN}}\right. \\
0 \cdot\left(\mathrm{cm}^{-2}\right) \text { otherwise }
\end{array} \\
& \mathrm{C}_{\mathrm{TMG}} \leftarrow \| \mathrm{C}_{\mathrm{TMG}}-\Delta \mathrm{C}_{\mathrm{GaN}} \text { if }\left(\mathrm{C}_{\mathrm{TMG}}-\Delta \mathrm{C}_{\mathrm{Ga}}\right. \\
& \text { time } \leftarrow \text { time }+\Delta \mathrm{t} \cdot \mathrm{s} \\
& \text { tim } \leftarrow \text { time } \cdot \mathrm{s}^{-1} \\
& \text { PRES } \leftarrow \text { PRESSURE }\left(\text { tim, }, P_{\text {tot }}\right) \cdot \text { mbar } \\
& \mathrm{C}_{\text {adduct }} \leftarrow \operatorname{COV} \text { (PRES) } \cdot \mathrm{C}_{\mathrm{TMG}} \\
& \text { SWITCH } \leftarrow 0 \\
& \mathrm{U}_{\mathrm{i}, 0} \leftarrow \text { time } \cdot \mathrm{s}^{-1} \\
& \mathrm{U}_{\mathrm{i}, 1} \leftarrow \mathrm{C}_{\mathrm{GaN}} \cdot \mathrm{cm}^{2}
\end{aligned}
$$


${ }_{\mathrm{K}}^{\mathrm{K}} \mathrm{aN}:=1 \times 10^{-16} \cdot \mathrm{cm}^{2} \mathrm{~s}^{-1}$

$$
\left.\right|_{u} \begin{aligned}
& \mathrm{U}_{\mathrm{i}, 2} \leftarrow \mathrm{C}_{\text {adduct }} \cdot \mathrm{cm}^{2} \\
& \mathrm{U}_{\mathrm{i}, 3} \leftarrow \mathrm{C}_{\mathrm{TMG}} \cdot \mathrm{cm}^{2} \\
& \mathrm{i} \leftarrow \mathrm{i}+1
\end{aligned}
$$

$$
\begin{aligned}
& C_{1 \mathrm{mbar}}:=\mathrm{BRIDGE}_{\mathrm{NH} 2 \text {.purge }}\left(\mathrm{t}, \Delta \mathrm{t}, \mathrm{k}_{\text {ads }}, \mathrm{k}_{\mathrm{des}}, \mathrm{k}_{\mathrm{GaN}}, 1 \times 10^{-0} \mathrm{mbar}\right) \mathrm{C}_{\mathrm{GaN}_{1} 1 \mathrm{mbar}_{\mathrm{i}}}:=\mathrm{C}_{1 \mathrm{mbar}}{ }_{\mathrm{i}, 1} \\
& C_{5 \mathrm{mbar}}:=\mathrm{BRIDGE}_{\mathrm{NH} 2 \text {.purge }}\left(\mathrm{t}, \Delta \mathrm{t}, \mathrm{k}_{\text {ads }}, \mathrm{k}_{\mathrm{des}}, \mathrm{k}_{\mathrm{GaN}}, 5 \times 10^{-0} \mathrm{mbar}\right) \mathrm{C}_{\mathrm{GaN} .5 \mathrm{mbar}_{\mathrm{i}}}:=\mathrm{C}_{5 \mathrm{mbar}_{\mathrm{i}, 1}} \\
& \mathrm{C}_{10 \mathrm{mbar}}:=\mathrm{BRIDGE}_{\mathrm{NH} 2 \text {.purge }}\left(\mathrm{t}, \Delta \mathrm{t}, \mathrm{k}_{\text {ads }}, \mathrm{k}_{\text {des }}, \mathrm{k}_{\mathrm{GaN}}, 10 \times 10^{-0} \mathrm{mbar} \mathrm{C}_{\mathrm{GaN} .10 \mathrm{mbar}_{\mathrm{i}}}:=\mathrm{C}_{10 \mathrm{mbar}_{\mathrm{i}, 1}}\right. \\
& \mathrm{C}_{20 \mathrm{mbar}}:=\mathrm{BRIDGE}_{\mathrm{NH} 2 \text {.purge }}\left(\mathrm{t}, \Delta \mathrm{t}, \mathrm{k}_{\text {ads }}, \mathrm{k}_{\mathrm{des}}, \mathrm{k}_{\mathrm{GaN}}, 20 \times 10^{-0} \mathrm{mbar}_{\mathrm{GaN}^{2} 20 \mathrm{mbar}_{\mathrm{i}}}:=\mathrm{C}_{20 \mathrm{mbar}} \mathrm{i}_{\mathrm{i}, 1}\right.
\end{aligned}
$$

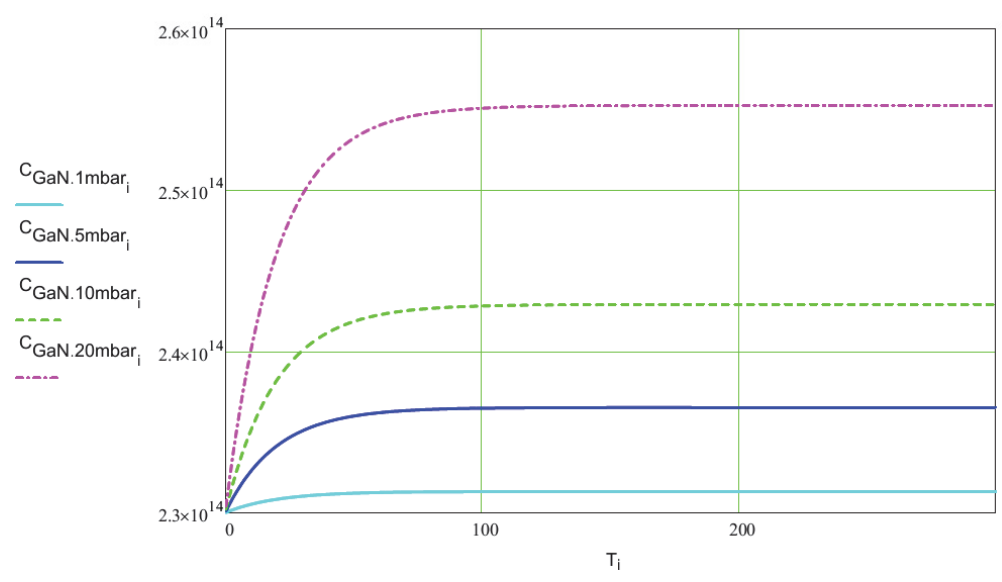




\section{6}

\section{Inherent substrate-selective thermal ALD of GaN*}

${ }^{*}$ This chapter is based on:

i. S. Banerjee, A. A. I. Aarnink, G. A. Kip, D. J. Gravesteijn, J. Schmitz and A. Y. Kovalgin, ALD Conf., Dublin, July 2016.

ii. S. Banerjee, A. A. I. Aarnink, D. J. Gravesteijn and A. Y. Kovalgin, EuroCVD 22-Baltic ALD 16 Conf., Luxembourg, June 2019.

iii. S. Banerjee, A. A. I. Aarnink and A. Y. Kovalgin, U.S. Patent App. 62779684, 2019. 


\subsection{Introduction}

The previous chapter introduced the thermal ALD mechanism of GaN, that was speculated to occur by the formation of the surface adduct between trimethylgallium (TMG) and ammonia $\left(\mathrm{NH}_{3}\right)$. The adduct converted into a $\mathrm{Ga}-\mathrm{NH}_{2}-\mathrm{Ga}$ linkage, resulting in the formation of a unit of GaN. This chapter will address the manner in which the ALD mechanism inherently facilitates substrate-selective growth of GaN. We hypothesize that the substrate-selective growth relies on the presence or absence of the $-\mathrm{NH}_{2}$ terminations on the growth surface ${ }^{*}$. We shall explore the ALD process at two pressure regimes: at low pressures (LP) between $10^{-3}-10^{-2}$ mbar, referred to as LPALD (Section 6.2) and at high pressures (HP) between 1-10 mbar (Section 6.3), referred to as HPALD. Whereas the growth kinetics studied in the previous chapter naturally leads to exploring the HP regime, selected experiments performed at the LP regime also demonstrate the influence of the $-\mathrm{NH}_{2}$ substrate terminations on the GaN ALD.

Accomplishing area-selective GaN growth is the logical next step in the ALD research in view of its potential benefits. For instance, due to the continued miniaturization of semiconductor devices, the control over conventional patterning techniques (etching, lithography, lift-off) is becoming excessively difficult ${ }^{1-2}$. In this aspect, area-selective ALD (ASALD) is highly relevant as it directly deposits the layer on the desired area, therefore eliminating the layer patterning step. Another promising application of ASALD is in the fabrication of fully self-aligned vias (FSAV) in the back end of line (BEOL) processes. This approach could reduce (or eliminate) via edge placement errors (EPE), and therefore mitigate some of the issues associated with EPE, such as, increased contact resistances, RCdelays and electromigration in modern microelectronic devices ${ }^{3}$. An approach to achieve ASALD is using a substrate with diverse terminations that are spatially separated. The terminations offer different nucleation rates for the precursors and selective film growth on certain areas can be achieved utilizing the nucleation delay ${ }^{4-6}$. In relation to the perceived role of the $-\mathrm{NH}_{2}$ terminations, initial experiments on ASALD of GaN were performed.

Section 6.2.1 compares the initial growth of GaN on H-terminated $\mathrm{Si}$ and in-situ deposited AlN buffer layer in LPALD, and Section 6.2.2 demonstrates the action of AlN monolayers in the LPALD. Section 6.3.1 addresses the different incubation phases on different substrates in HPALD, where the substrates contain or lack the $\mathrm{NH}_{2}$ terminations. Section 6.3.2 discusses control experiments performed to further address the role of these

\footnotetext{
* There exists a subtle difference between the definitions of $-\mathrm{NH}_{2}$ and $-\mathrm{NH}_{2}-$. The former implies ligands that terminate a surface (e.g., a $\mathrm{SiO}_{2}$ substrate after exposure to $\mathrm{NH}_{3}$-plasma). The latter implies the $\mathrm{Ga}-\mathrm{NH}_{2}-\mathrm{Ga}$ linkage through which the GaN ALD is proposed to occur. However, for buffer AlN and $\mathrm{GaN}$ layers, the two definitions are equivalent, meaning that their surfaces are terminated with $\mathrm{Al}-\mathrm{NH}_{2}-\mathrm{Al}$ and $\mathrm{Ga}-\mathrm{NH}_{2}-\mathrm{Ga}$, respectively.
} 
terminations, and also shows how the incubation phase is affected in the PEALD of GaN. Section 6.4 investigates the in-situ SE monitored growth pattern at different stages of the HPALD on several substrates, and explains the observations by the ALD model. Section 6.5 discusses the initial results obtained on the ASALD of GaN in the high pressure regime. Section 6.6 concludes the chapter.

\subsection{Low pressure (LP) ALD}

\subsubsection{GaN growth enhancement by ALD AIN buffer layer}

The ALD of GaN was attempted in the LP regime on pre-treated $\mathrm{Si}$ and $\mathrm{SiO}_{2}$ substrates and in-situ-deposited $\mathrm{AlN}$ buffer layer, at $400{ }^{\circ} \mathrm{C}$. The $\mathrm{Si}$ and $\mathrm{SiO}_{2}$ substrates were pre-treated respectively, with HF solution (1\%-by-volume) and ozone-steam. The pretreatment furnished these substrates with $-\mathrm{H}$ and $-\mathrm{OH}$ terminations. The AlN buffer layer was thermally deposited using a standard ALD recipe to a thickness of $70 \mathrm{~nm}$, at $350{ }^{\circ} \mathrm{C}$. Fig. 6.1 a shows the GaN growth, acquired by in-situ spectroscopic ellipsometry, on the $\mathrm{Si}$ and AIN substrates. Whereas the Si substrate hardly caused GaN formation even after many ALD cycles, the AIN buffer layer caused GaN growth, but with a very low GPC $(0.001 \mathrm{~nm} /$ cycle). The ALD recipe for both experiments was the same and can be found in the figure caption.

As Fig. 6.1 b reveals, the GaN GPC on the AlN layer reduced steadily with the progression of the ALD. This suggests: compared to on the $-\mathrm{NH}_{2}$ terminated AIN surface ${ }^{\dagger}$, the further $\mathrm{GaN}$ formation on the pre-formed $\mathrm{GaN}$ monolayers is hardly facilitated in the LP regime. This is because the formation of the $\mathrm{TMG}: \mathrm{NH}_{3}$ surface adduct hardly occurs at such low pressures (see Chapter 5). However, the initial GaN formation could have occurred due to the influence of the $-\mathrm{NH}_{2}$-terminated AlN surface. The evidence of the formation a thin GaN film was confirmed by XPS.

\footnotetext{
$\dagger$ The AlN ALD is reported to occur through the successive formation of $\mathrm{Al}-\mathrm{NH}_{2}-\mathrm{Al}$ linkages. Therefore, its surface is expected to be terminated by $-\mathrm{NH}_{2}$ groups (see Chapter $\mathbf{5}$ for details).
} 

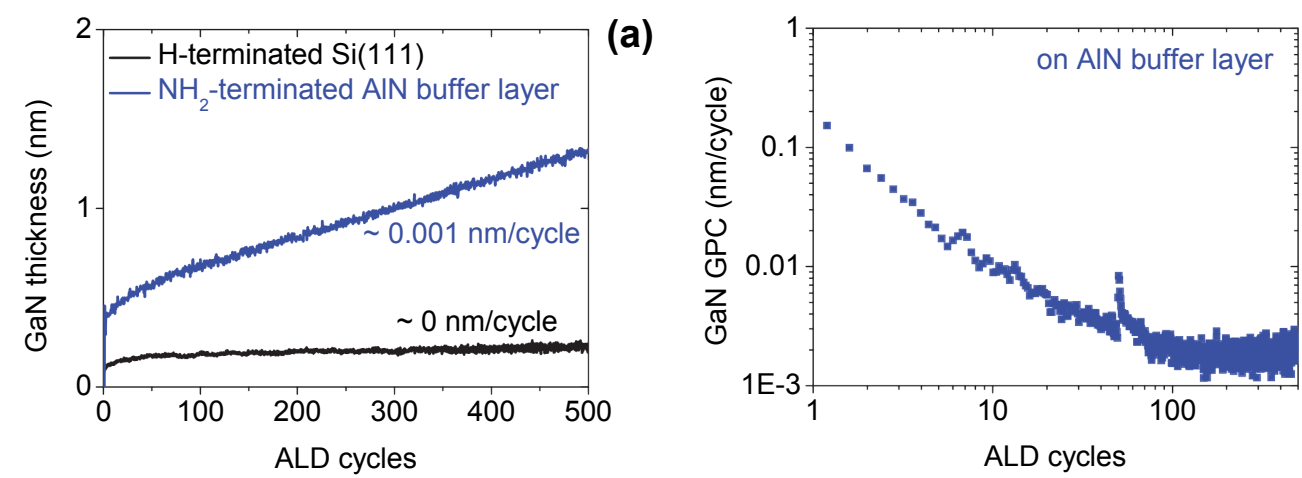

(b)

Fig. 6.1 (a) LPALD of GaN on $\mathrm{H}$-terminated $\mathrm{Si}$ substrate (in black) and $\mathrm{NH}_{2}$-terminated AlN buffer layer (in blue). Both the experiments were performed at $400^{\circ} \mathrm{C}$ and $7 \times 10^{-3} \mathrm{mbar} \mathrm{NH}_{3}$ partial pressure $\left(P_{\mathrm{NH} 3}\right)$. The ALD recipe was $0.1 \mathrm{~s}$ TMG $/ 4 \mathrm{~s} \mathrm{Ar} / 2 \mathrm{~s} \mathrm{NH}_{3} / 4 \mathrm{~s}$ Ar. (b) Evolution of GaN GPC on the AlN buffer layer.

\subsubsection{GaN growth enhancement by AIN monolayers deposited within super-cycles}

If a surface is terminated with $-\mathrm{NH}_{2}$, it will presumably facilitate the initial chemisorption of TMG, as the previous experiment highlighted. Therefore, the deposition of a thick buffer layer becomes redundant. To test this assumption, an ALD 'super-cycle' recipe containing an AlN growth phase and a GaN growth phase was designed. Each super-cycle consisted of $m$ sub-cycles of TMA and $\mathrm{NH}_{3}$ pulses, and $n$ sub-cycles of TMG and $\mathrm{NH}_{3}$ pulses. The complete ALD cycle is expressed as $\left\{m\right.$ (TMA pulse/ purge / $\mathrm{NH}_{3}$ pulse / purge) $+n$ (TMG pulse / purge / $\mathrm{NH}_{3}$ pulse / purge) $\}^{\star}$. Experiments were performed with values of $m$ and $n$ ranging between 1 and 15. The action of the as-deposited AlN (sub-)monolayers during the $m$ sub-cycles, on the GaN formation during the $n$ sub-cycles, was investigated as further described.

In-situ monitoring the layer growth revealed distinct changes in the growth curve during the AlN and GaN phases (Fig. 6.2). Although the SE acquisition was not synchronized with respect to the precursor pulses, the two phases were identified from their precise match with the sub-cycle durations. Fig. 6.2 shows that whereas the growth is enhanced in every AlN phase, it reduces in every GaN phase. This can be explained by considering that the AlN (sub-)monolayers form easily at low pressures, because the TMA: $\mathrm{NH}_{3}$ surface adduct formation is less pressure-dependent (see Chapter 5). However, after an initial TMG nucleation and speculatively, formation of a few $\mathrm{GaN}$ monolayers, the further $\mathrm{GaN}$ growth is hardly sustained at the low pressure since the TMG:NH 3 surface

\footnotetext{
$\$$ The $m$ and $n$ sub-cycles are also referred to as the 'AlN phase' and 'GaN phase', respectively.
} 
adduct formation is strongly pressure-dependent. The result is thus qualitatively similar to Fig. 6.1 b.

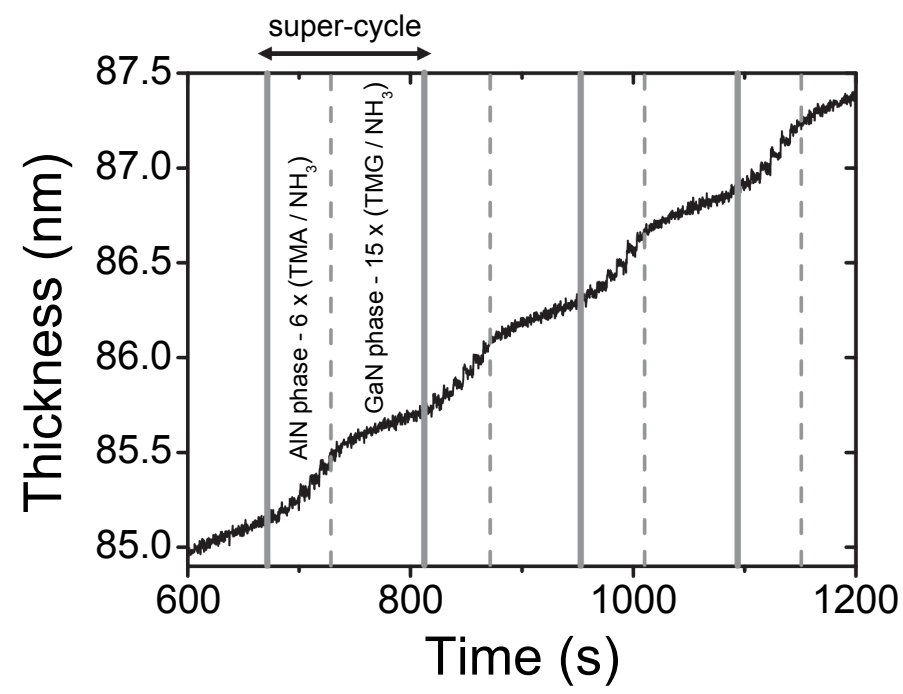

Fig. 6.2 Growth in the super-cycle ALD recipe: $\left\{6 \times\left(0.5 \mathrm{~s}\right.\right.$ TMA / $\left.2 \mathrm{~s} \mathrm{Ar} / 2.5 \mathrm{~s} \mathrm{NH}_{3} / 4 \mathrm{~s} \mathrm{Ar}\right)+$ $\left.15 \times\left(0.1 \mathrm{~s} \mathrm{TMG} / 2 \mathrm{~s} \mathrm{Ar} / 1 \mathrm{~s} \mathrm{NH}_{3} / 2.5 \mathrm{~s} \mathrm{Ar}\right)\right\}$, performed at $400{ }^{\circ} \mathrm{C}$ and $10^{-3} \mathrm{mbar} P_{\mathrm{NH} 3}$. The solid lines show the duration of 1 super-cycle, and the dotted lines show the AIN and GaN phases within a super-cycle.

Using a super-cycle of $m=1$ and $n=1$ on H-terminated $\mathrm{Si}$, we can similarly distinguish between the two phases (Fig. 6.3 a). The clear and repeated occurrence of the 'step' during every GaN phase shows that TMG chemisorption is possible even on the AlN (sub-)monolayer formed by a single TMA-and- $\mathrm{NH}_{3}$ pulse.

The results of Fig. 6.2 and Fig. 6.3 a were obtained when the overall layer had become thick (63 and $86 \mathrm{~nm}$, respectively). In the experiment of Fig. 6.3 a, to find out whether TMG could nucleate on AIN monolayers from the beginning of the deposition (i.e., directly on the Si substrate), depth-profiled XPS was performed (Fig. 6.3 b). From the already-constant $\mathrm{Ga}$ content, along with constant $\mathrm{Al}$ and $\mathrm{N}$ contents directly from the substrate, we conclude that the TMG chemisorption could occur on AIN (sub-)monolayers from the very beginning. This resulted in a constant Ga incorporation in every super-cycle. The attainment of a certain critical layer thickness, after which TMG could chemisorb on AlN, was therefore not required. However, without the (sub-)AlN monolayers, the chemisorption of TMG directly on the Si substrate is hardly facilitated, as previously shown in Fig. 6.1 a. 
Furthermore, the amount of AlN deposited in the AlN phase (i.e., $m$ ) was found to affect the amount of $\mathrm{GaN}$ deposited in the GaN phase, for fixed $n$. Table 6.1 compares the thickness increase observed in the GaN phase for $n$ of 15 , from $m$ of 1,6 and 1000 . The bigger thickness-increase for larger $m$ is explained by the higher surface coverage of the $-\mathrm{NH}_{2}$ terminations on the AlN, supporting higher coverage of the chemisorbed TMG. This observation (made in the super-cycle ALD recipe) is also in accordance with the direct dependence of the TMG coverage on the $-\mathrm{NH}_{2}$ surface coverage (where the $-\mathrm{NH}_{2}$ are from GaN monolayers instead) reported in the previous chapter (Section 5.7).
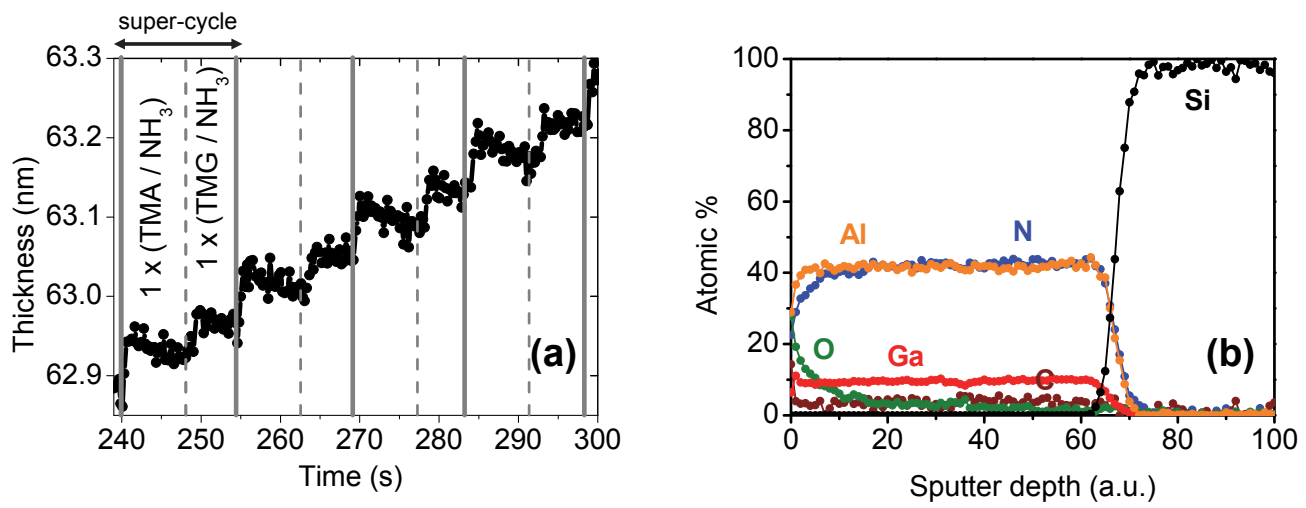

Fig. 6.3 (a) Layer growth in the AlN and GaN phases in the super-cycle ALD recipe: $\{1(0.5 \mathrm{~s}$ TMA / $\left.\left.2 \mathrm{~s} \mathrm{Ar} / 2.5 \mathrm{~s} \mathrm{NH}_{3} / 4 \mathrm{~s} \mathrm{Ar}\right)+1\left(0.1 \mathrm{~s} \mathrm{TMG} / 2 \mathrm{~s} \mathrm{Ar} / 1 \mathrm{~s} \mathrm{NH}_{3} / 2.5 \mathrm{~s} \mathrm{Ar}\right)\right\}$, performed at $400{ }^{\circ} \mathrm{C}$ and $10^{-3}$ mbar $P_{\mathrm{NH} 3}$. (b) Depth profiled XPS of the same layer.

Table 6.1 Average thickness increase recorded during the $\mathrm{GaN}$ phase of the super-cycle $(n=15)$ for different number $(m)$ of AlN phases. To note: the thicknesses are not exact for these ultra-low values, but only give a qualitative indication.

\begin{tabular}{|c|c|c|}
\hline $\boldsymbol{m}$ & $\boldsymbol{n}$ & $\begin{array}{c}\text { Average thickness } \\
\text { increase during } \\
\boldsymbol{n} \mathrm{TMG}_{\mathbf{N M H}} \text { pulses }\end{array}$ \\
\hline 1 & 15 & $0.11 \mathrm{~nm}$ \\
\hline 6 & 15 & $0.29 \mathrm{~nm}$ \\
\hline $\begin{array}{c}1000 \\
(70 \mathrm{~nm} \\
\text { AIN buffer })\end{array}$ & 15 & $0.51 \mathrm{~nm}$ \\
\hline
\end{tabular}

In summary, the LPALD results show that as long as $-\mathrm{NH}_{2}$ terminations are present, whether on a thick AlN buffer layer or on ultra-thin AlN (sub-)monolayers, TMG chemisorbs to them even at the low pressures with relative ease. However, the further GaN formation is not well facilitated due to the inefficiency of the surface adduct formation. 


\subsection{High-pressure (HP) ALD}

In the HP regime, the influence of the $-\mathrm{NH}_{2}$ terminations ${ }^{\S}$ on GaN ALD is revealed from the nature of its initial growth on various substrates. The substrates are either terminated by, or devoid of the $-\mathrm{NH}_{2}$ terminations. Among the first category are in-situ deposited ALD AlN and CVD GaN buffer layers, and among the second category are $\mathrm{H}$-terminated $\mathrm{Si}$ and $\mathrm{OH}$-terminated $\mathrm{SiO}_{2}$ substrates.

\subsubsection{Dependence of incubation time on surface termination}

Fig. 6.4 shows the in-situ monitored HPALD GaN growth on $\mathrm{Si}$ and $\mathrm{SiO}_{2}$ substrates, and on AlN and GaN buffer layers. The incubation time* is remarkably different on these two substrate categories. Whereas $\mathrm{Si}$ and $\mathrm{SiO}_{2}$ provide a long and an equal incubation time (140 cycles), AlN and GaN provide a much shorter incubation time (20 cycles).

The short incubation time on the $-\mathrm{NH}_{2}$ terminated AlN and GaN buffer layers implies that the chemisorption of TMG and its conversion into GaN (to note: the surface adduct formation occurs with ease at the high pressures) are well-facilitated on these layers, compared to that on $\mathrm{Si}$ and $\mathrm{SiO}_{2}$. As the ALD mechanism involves TMG chemisorbing on the $-\mathrm{NH}_{2}$ - linkages, the presence of similar ligands on the surface is expected to initiate the growth readily. The remarkably similar growth patterns on AlN and GaN imply similar surface terminations (i.e., $-\mathrm{NH}_{2}$ ) on both, and confirm once more the growth model of Chapter 5.

On the contrary, the absence of $-\mathrm{NH}_{2}$ terminations on the substrate demands multiple ALD cycles to furnish it with the terminations required to support the TMG chemisorption and $\mathrm{GaN}$ formation. This is reflected by the long incubation time on both $\mathrm{Si}$ and $\mathrm{SiO}_{2}$. The growth mechanism at the different ALD stages on AlN and $\mathrm{Si}$ is further addressed in Section 6.4.

Fig. 6.4 (in dotted lines) reveals that the GPC stabilizes at $0.09 \mathrm{~nm} /$ cycle after 50 ALD cycles on AlN and GaN, and after 250 cycles on $\mathrm{Si}$ and $\mathrm{SiO}_{2}$. Prior to reaching this stage, the GPC had increased monotonically. The stabilization may imply that the substrate had acquired a constant coverage of $-\mathrm{NH}_{2}-$ linkages, which likewise support a constant

\footnotetext{
$\S$ Although not directly confirmed by corresponding surface-analysis techniques, it is hypothesized that the $-\mathrm{NH}_{2}$ terminations naturally occur based on the growth mechanism proposed in Chapter 5.

** The incubation time is defined here as the number of ALD cycles required to grow $1 \mathrm{~nm}$ of GaN.
} 
coverage of the chemisorbed TMG. After the stabilization, the growth on every substrate occurs with a GPC of $0.09 \mathrm{~nm} /$ cycle.

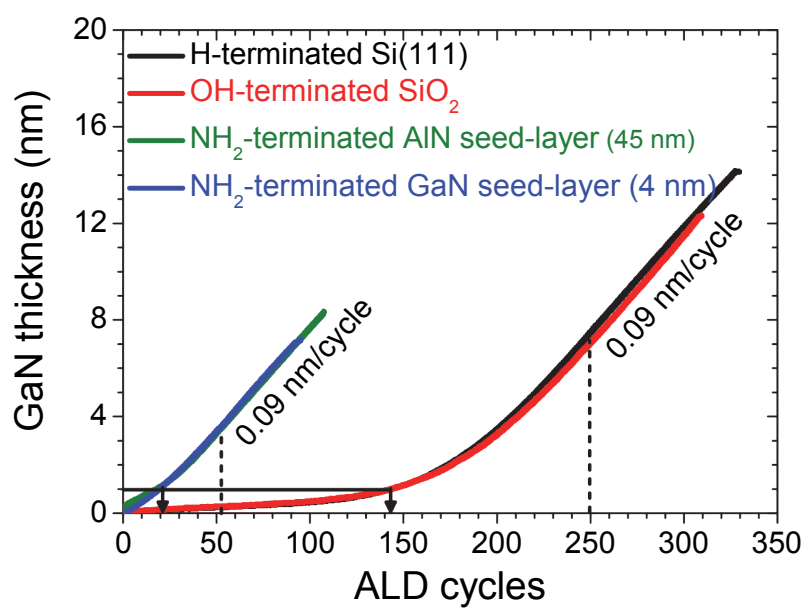

Fig. 6.4 GaN thickness evolution on $-\mathrm{NH}_{2}$ terminated substrates (AIN and $\mathrm{GaN}$ buffer layers) and non- $\mathrm{NH}_{2}$-terminated substrates $\left(\mathrm{Si}\right.$ and $\left.\mathrm{SiO}_{2}\right)$, showing different incubation times. The ALD recipe for every experiment was: $0.1 \mathrm{~s} \mathrm{TMG} \mathrm{/} 30 \mathrm{~s} \mathrm{Ar} / 30 \mathrm{~s} \mathrm{NH}_{3} / 60 \mathrm{~s} \mathrm{Ar}$, at $400{ }^{\circ} \mathrm{C}$ and 1.3 mbar $P_{\mathrm{NH} 3}$. The duration of incubation is shown by the solid arrows. The stage at which the GPC stabilizes is shown by the dotted lines.

\subsubsection{Control experiments showing the role of $-\mathrm{NH}_{2}$ terminations and the effect of plasma}

The influence of the $-\mathrm{NH}_{2}$ terminations on initial $\mathrm{GaN}$ growth was confirmed in the following control experiments. In the first experiment, a $\mathrm{SiO}_{2}$ substrate was in-situ annealed in a stream of continuously flowing $\mathrm{NH}_{3}$ (at $600{ }^{\circ} \mathrm{C}, 45 \mathrm{~min}$ ), to presumably furnish the substrate with the $-\mathrm{NH}_{2}$ terminations. This indeed reduced the incubation phase from 140 (recall Fig. 6.4) to about 80 cycles (Fig. 6.5 a, cyan).

The second experiment demonstrated that oxidizing the $-\mathrm{NH}_{2}$ terminations prolonged the incubation time. A H-terminated $\mathrm{Si}$ wafer was treated with $\mathrm{NH}_{3}$ plasma (for $45 \mathrm{~min}$ ), to furnished it with $-\mathrm{NH}_{2}$ terminations. The plasma-treated substrate was subsequently exposed to air (for $30 \mathrm{~min}$ ) and re-introduced into the ALD reactor. Remarkably, the incubation phase of 160 cycles (Fig. 6.5 a, grey) was similar to that on the $\mathrm{Si}$ and $\mathrm{SiO}_{2}$ substrates observed before, which confirmed the interaction of the terminations with air, presumably, their oxidation. 
Conversely to the thermal ALD results, PEALD of GaN using TMG and $\mathrm{NH}_{3}$ plasma on both $\mathrm{Si}$ and $\mathrm{SiO}_{2}$ substrates resulted in a short incubation time of 25 cycles (Fig. 6.5 b). This value is similar to that offered by the in-situ deposited AlN and GaN buffer layers in thermal ALD (Fig. 6.4). This could be because the plasma furnished the substrates with $-\mathrm{NH}_{2}$ or $-\mathrm{NH}$ terminations, initiating the GaN growth.
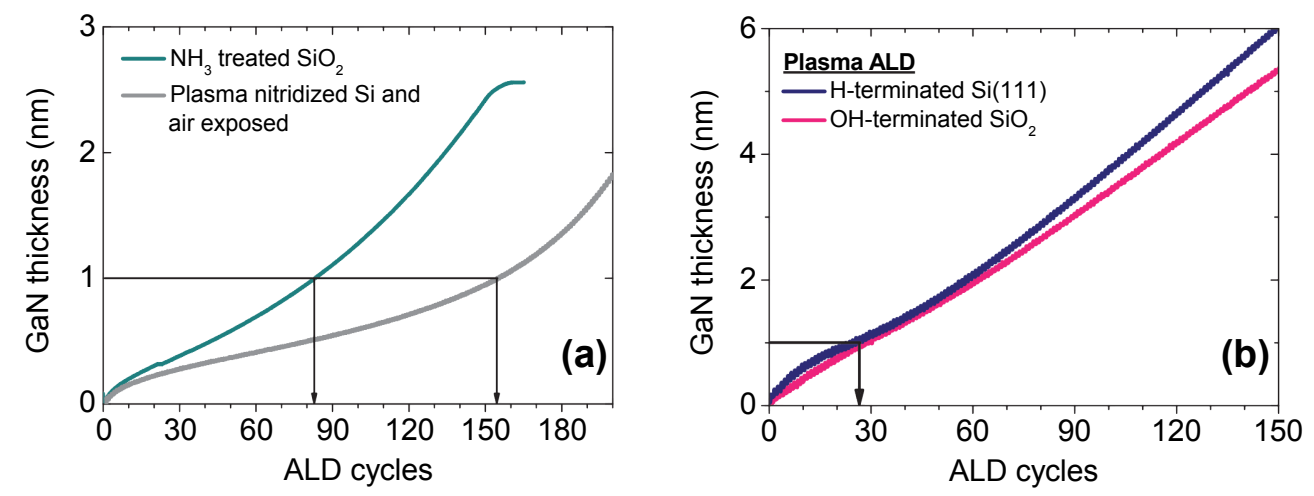

Fig. 6.5 GaN thickness evolution (a) during thermal $\mathrm{ALD}$ on $\mathrm{NH}_{3}$-annealed $\mathrm{SiO}_{2}$ substrate (in cyan) and on Si substrate that was plasma-treated and air-exposed (in grey). The ALD recipe is the same as that of Fig. 6.4. (b) Thickness evolution on $\mathrm{Si}$ and $\mathrm{SiO}_{2}$ substrates during PEALD of GaN from TMG and $\mathrm{NH}_{3}$-plasma.

\subsection{Investigation into the growth on Si and AIN substrates by in-situ SE}

Monitored by in-situ SE, the ALD of GaN is revealed not to occur in a continuous manner, but through a series of well-defined 'steps' and 'plateaus' (Fig. 6.6 a), as introduced in the previous chapter (Section 5.7). The step follows the TMG pulse, and the plateau contains the remaining part of the ALD cycle. The step is ascribed to the chemisorption of TMG (presumably, on the $-\mathrm{NH}_{2}-$ linkages terminating the underlying pre-formed $\mathrm{GaN}$ layer), and the plateau is ascribed to the formation of the adduct and its conversion into the subsequent $-\mathrm{NH}_{2}-$ linkage. The height of the step gives an indication of the surface coverage of the chemisorbed TMG and the $-\mathrm{NH}_{2}-$ linkages.

Fig. 6.6 a shows the GaN growth pattern after the initial growth stage; i.e., after the GPC is stabilized. Consequently, the step-heights are relatively constant at every ALD cycle. This stable-step-height regime is not reached immediately on $\mathrm{Si}$ (or $\mathrm{SiO}_{2}$ ) and on $\mathrm{AlN}$ (or $\mathrm{GaN}$ ) surfaces, but after a number of ALD cycles depending on the presence or the absence of $-\mathrm{NH}_{2}$ terminations. For example, Fig. 6.6 b, c shows the GaN growth on a H-terminated Si substrate at an earlier and a later ALD stage, respectively. The step height increases by up to 4 times from the $140^{\text {th }}$ cycle to the $300^{\text {th }}$ cycle. Fig. 6.7 a, b shows the evolution of the 
step height on the $-\mathrm{H}$ terminated $\mathrm{Si}$ and a $-\mathrm{NH}_{2}$ terminated $\mathrm{AlN}$ substrate for the complete ALD process $^{\dagger \dagger}$. The different ALD stages will be analysed below.

\subsubsection{The incubation stage}

On H-terminated $\mathrm{Si}$, for up to about 140 ALD cycles the step-height is quite low (Fig. 6.7 a). The layer growth during this stage is shown in Fig. 6.6 b. During this so-called 'incubation stage', the low step height reflects a low surface coverage of the chemisorbed TMG. This is because the chemisorption is inhibited on the H-terminated substrate. The low surface coverage of the chemisorbed TMG in turn leads to a low coverage of the $-\mathrm{NH}_{2}-$ linkages. During this stage, isolated GaN and Ga clusters are speculated to form. In contrast, the incubation period lasts for only about 20 cycles on the $-\mathrm{NH}_{2}$-terminated $\mathrm{AlN}$ (see Fig. 6.4) .

\subsubsection{The stage of increasing step height}

With the progression of the ALD, since TMG starts to chemisorb on the alreadyformed GaN clusters, and also on the other unoccupied areas of the substrate, its surface coverage increases and consequently the step-height does ${ }^{\star *}$. This phase is between $140-250$ cycles on Si (Fig. 6.7 a), and between $20-50$ cycles on AlN (Fig. 6.7 b).

\subsubsection{The stage of stable step height}

The attainment of a stable-step-height stage, which occurs after 250 cycles on $\mathrm{Si}$ and 50 cycles on AlN, suggests that the growing GaN surface finally achieved a steady coverage of $-\mathrm{NH}_{2}-$ terminations, and these are capable of supporting a constant coverage of the chemisorbed TMG. This stage may also imply the closure of the GaN layer, i.e., completely covering the substrate. For the remaining ALD process, the step-height remains a constant on both Si and AlN. This stage is reached earlier on AlN due to the already-present $-\mathrm{NH}_{2}$ terminations, supporting a high surface coverage of the chemisorbed TMG already

† See Chapter 5, Section 5.7 w.r.t replacing absolute thicknesses by relative thicknesses.

$\$$ Since the proposed ALD model requires adjacent chemisorbed TMG units to form the $-\mathrm{NH}_{2}-$ linkages, the greater the number of TMG molecules chemisorbing on adjacent substrate sites, the higher is the chance of the adduct and linkage formation. The linkages can then accommodate newer TMG species (on the contrary, isolated TMG clusters can dissociate into Ga, without converting into $\mathrm{GaN}$ ). Such second-order effects additionally contribute to the evolution of the step height. 
from the initial ALD stages. The evolution of the step height on $\mathrm{SiO}_{2}$ is qualitatively similar to that on $\mathrm{Si}$; likewise, the evolution on the CVD GaN buffer-layer is similar to than on AlN.
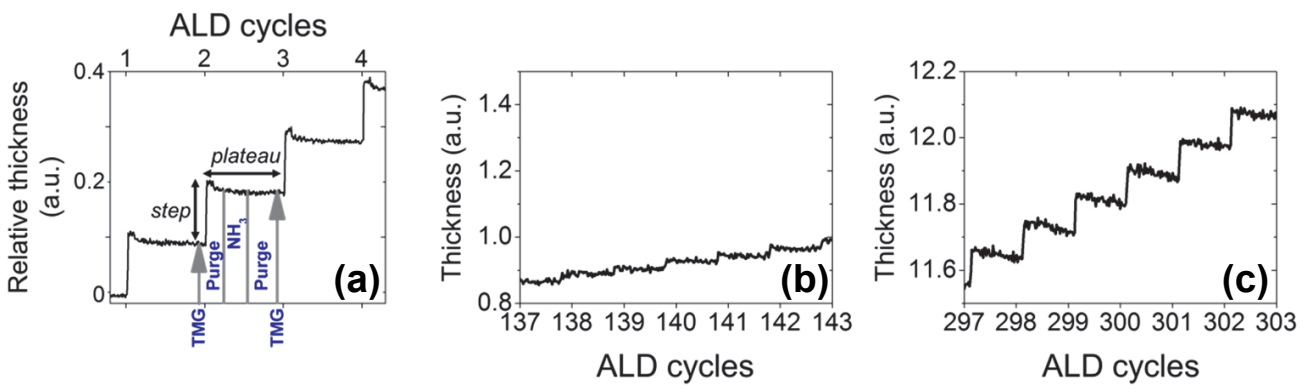

Fig. 6.6 (a) GaN growth through repeated sequence of 'steps' and 'plateaus', as monitored by in-situ SE. (b, c) Small and large step heights observed around the $140^{\text {th }}$ and $300^{\text {th }}$ cycles, respectively, of the same ALD process.
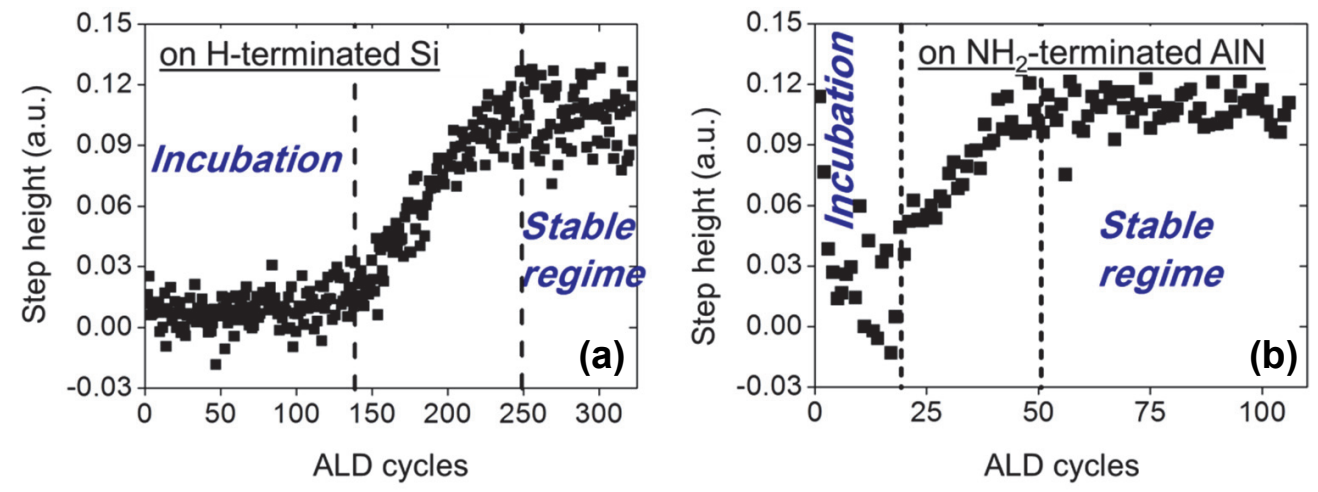

Fig. 6.7 Evolution of step height with ALD cycles on (a) H-terminated Si substrate, (b) $-\mathrm{NH}_{2}$ terminated AIN substrate. The incubation and the stable step-height regimes correspond to Fig. 6.4.

\subsection{Towards thermal ASALD of GaN}

The large differences in the incubation times on the $-\mathrm{NH}_{2}$ terminated and the non $-\mathrm{NH}_{2}$ terminated substrates (i.e., the 'substrate-selective' aspect of the GaN ALD), can be utilized to achieve the ASALD. The targeted application is in the industry where ex-situ patterned surfaces are used. When the growth surface consists of spatially separated regions of $-\mathrm{NH}_{2}$ and non- $\mathrm{NH}_{2}$ terminations, $\mathrm{GaN}$ growth can be accomplished selectively on the former region. However, it is important to verify whether the terminations remain chemically unaffected by the ex-situ patterning. This section will discuss two initial experiments towards achieving the ASALD of GaN. 


\subsubsection{ALD on patterned $\mathrm{AIN} / \mathrm{SiO}_{2}$ substrate}

After the deposition of a $54 \mathrm{~nm}$ thick ALD AlN layer on $\mathrm{SiO}_{2}$ substrate, the layer was patterned by photolithography and etching (the etching recipe can be found in Appendix 6.1). Thereafter, GaN ALD was performed on the patterned substrate. In-situ SE was used to monitor the growth on a patterned AlN surface of $1 \times 1 \mathrm{~cm}^{2}$ (Fig. 6.8 a). The incubation time was 160 cycles. This value was noticeably higher than on the in-situ deposited AlN layer, but similar to that on $\mathrm{Si}$ or $\mathrm{SiO}_{2}$ substrates (recall Fig. 6.4). The air-exposure of the AlN and/or the lithography and etching steps obviously changed the properties of the $-\mathrm{NH}_{2}$ terminations.
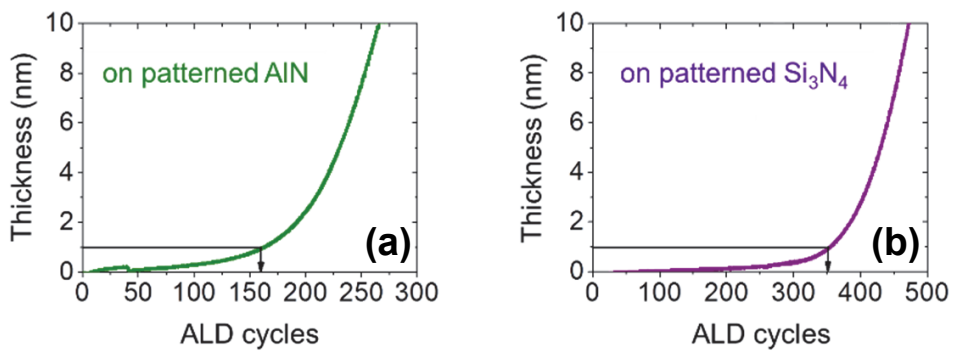

Fig. 6.8 GaN thickness evolution on (a) patterned $\mathrm{AlN}$ and (b) patterned $\mathrm{Si}_{3} \mathrm{~N}_{4}$ surfaces. The patterns were $1 \mathrm{x} 1 \mathrm{~cm}^{2}$ in size.

Fig. 6.9 shows a combination of electron microscopy images (energy selective backscattered (ESB) and energy dispersive X-ray (EDX)), showing the outcome of the GaN ALD on $\mathrm{SiO}_{2}$ and $\mathrm{AlN}$ areas. In the ESB image, since the under-layers $\left(\mathrm{AlN}, \mathrm{SiO}_{2}\right)$ have different contrasts, it is impossible to make any conclusion about the deposited layer. However, EDX detects Ga signal from both the areas, whereas $\mathrm{N}$ from only the AlN part. The latter could also be the effect of the underlying AIN layer, since the deposited layer is rough and thin (15 - $20 \mathrm{~nm}$ on both $\mathrm{AlN}$ and $\mathrm{SiO}_{2}$, as shown in Appendix 6.2).

A more convincing analysis is provided by ex-situ SE, acquired from the AlN and $\mathrm{SiO}_{2}$ areas (Fig. 6.10 a, b). For the former, the layer stack was modelled as $\mathrm{Si} / \mathrm{SiO}_{2} / \mathrm{AlN} /$ GaN. The $\mathrm{SiO}_{2}$ and $\mathrm{AlN}$ layers were modelled by a Cauchy optical model with their thicknesses measured before and after the AIN ALD, respectively. The deposited layer was also modelled by Cauchy (assuming the optical constants of $\mathrm{GaN}$ ) and yielded an acceptable fit accuracy (Fig. 6.10 a). However, when the same model but without the AlN layer was applied on the $\mathrm{SiO}_{2}$ area, the fit quality drastically reduced (Fig. 6.10 b). This gives an indication that the layer deposited on the $\mathrm{SiO}_{2}$ is different from that deposited on the AlN. Corresponding to the EDX result, the former could indeed be Ga, and the latter could be $\mathrm{GaN}$. 


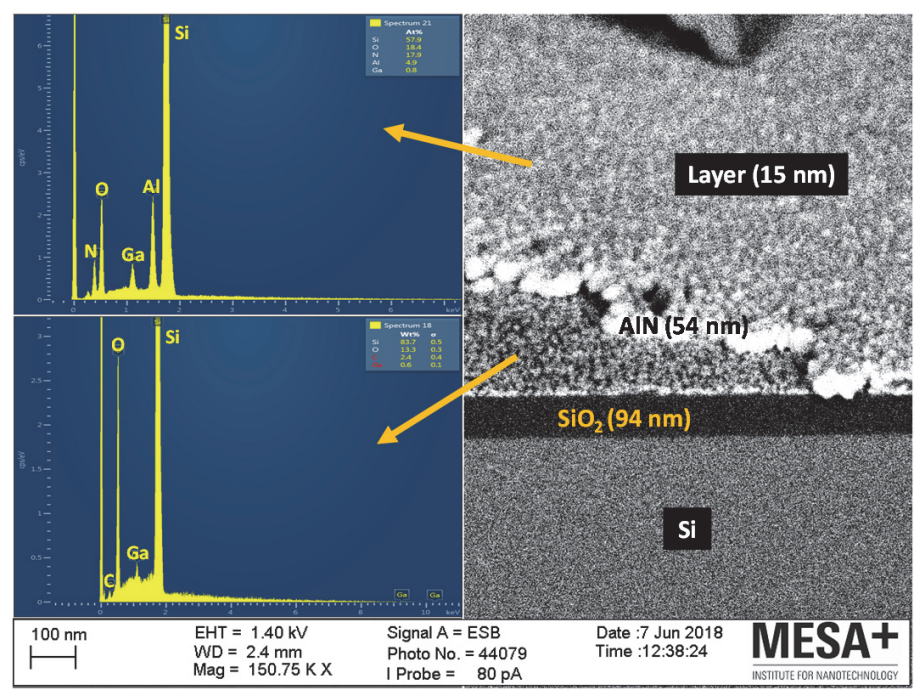

Fig. 6.9 EDX (left) and ESB (right) results of GaN ALD performed on patterned $\mathrm{SiO}_{2} / \mathrm{AlN}$ surfaces. (Due to its unknown composition, the deposited layer is not named.)

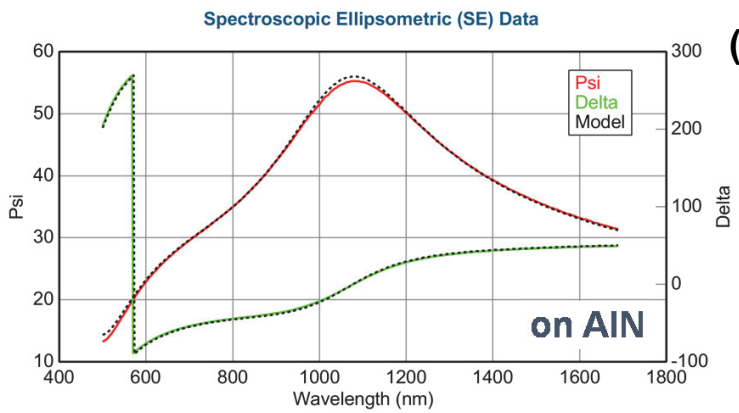

(a)

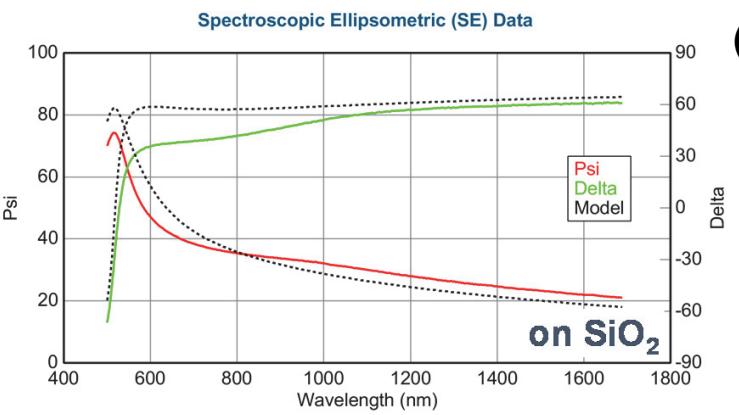

(b)

Fig. 6.10 Psi-Delta values obtained by $\mathrm{SE}$ from (a) $\mathrm{AlN}$ and (b) $\mathrm{SiO}_{2}$ areas. The dotted line shows the fit generated by a $\mathrm{Si} / \mathrm{SiO}_{2} / \mathrm{AlN} / \mathrm{GaN}$ optical model for a, and a $\mathrm{Si} / \mathrm{SiO}_{2} / \mathrm{GaN}$ model for $\mathbf{b}$. The better fit in the first case suggests the formation of GaN on the AlN area. 


\subsubsection{ALD on patterned $\mathrm{Si}_{3} \mathrm{~N}_{4} / \mathrm{SiO}_{2} / \mathrm{Si}$ substrate}

After depositing and patterning a $45 \mathrm{~nm} \mathrm{LPCVD} \mathrm{Si}_{3} \mathrm{~N}_{4}$ layer on a pre-patterned $\mathrm{Si} / \mathrm{SiO}_{2}$ substrate (Fig. 6.11 a), GaN ALD was performed. The growth was in-situ monitored on the $\mathrm{Si}_{3} \mathrm{~N}_{4}$ area (Fig. 6.8 b) and shows an extremely long incubation time of 350 cycles. Because no noticeable difference in the layer thickness was obtained on all the three surfaces $\left(\mathrm{Si}_{3} \mathrm{~N}_{4}, \mathrm{SiO}_{2}, \mathrm{Si}\right.$ ) (Fig. 6.11 b - d), it is likely that in terms of nucleation there is no significant difference in surface terminations after the patterning steps. This result again demonstrates the sensitivity of the ALD to substrate termination, and motivates the need of further research towards preserving spatially-separated regions of $-\mathrm{NH}_{2}$ terminations during ex-situ patterning. The observed difference between the incubation times of the two experiments in Fig. 6.8 a (and Appendix 6.2) and Fig. 6.8 b can be directly exploited for $\mathrm{AlN} / \mathrm{Si}_{3} \mathrm{~N}_{4}$ substrate-selective growth.

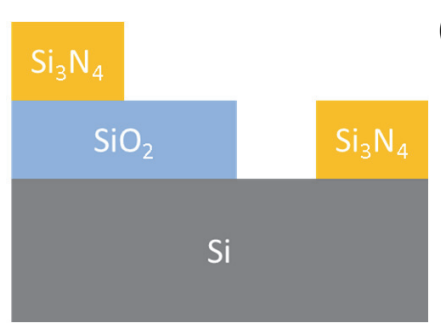

(a)
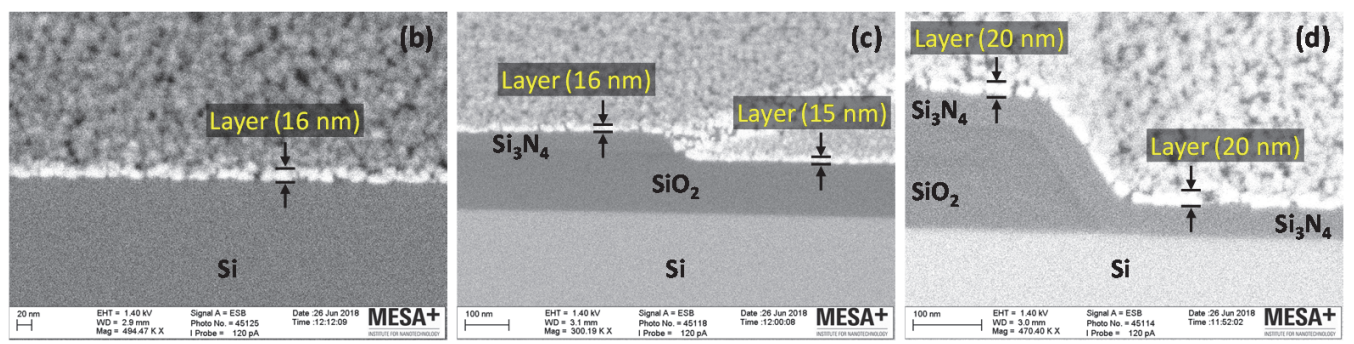

Fig. 6.11 (a) Schematic of the $\mathrm{Si} / \mathrm{SiO}_{2} / \mathrm{Si}_{3} \mathrm{~N}_{4}$ structure. (b - d) ESB images showing the result of the ALD on the three surfaces. No noticeable differences in the layer thicknesses were observed. (Due to its unknown composition, the deposited layer is not named.) 


\subsection{Conclusions}

The importance of $-\mathrm{NH}_{2}$ terminations in GaN ALD was demonstrated at both low (LP) and high (HP) pressures. In LPALD, in-situ deposited AlN buffer layers and AlN (sub-)monolayers obtained within super-cycles showed the same result: an initial chemisorption of TMG could occur on the $-\mathrm{NH}_{2}$ surface terminations of AlN, but the subsequent GaN formation was not facilitated, presumably due to the inefficiency of the surface adduct formation.

In HPALD, a long incubation stage (140 cycles) was observed on both the Hterminated $\mathrm{Si}$ and $\mathrm{OH}$-terminated $\mathrm{SiO}_{2}$ substrates. On the contrary, significantly shorter incubation stage $\left(20\right.$ cycles) was observed on in-situ deposited and presumably $\mathrm{NH}_{2}$ terminated $\mathrm{AlN}$ and $\mathrm{GaN}$ buffer layers. Beyond 250 cycles on $\mathrm{Si}\left(\right.$ and $\mathrm{SiO}_{2}$ ), and 50 cycles on AlN (and GaN), the GPC stabilized at the same constant value on all the substrates, suggesting a steady coverage of $-\mathrm{NH}_{2}-$ linkages. Such observations were further investigated by focussing on the evolution of the step heights at different stages of the ALD.

Exploiting the inherently different incubation times of $\mathrm{GaN}$ depending on the presence or absence of the $-\mathrm{NH}_{2}$ surface terminations, the area-selective aspect of GaN ALD was explored. Patterned $\mathrm{AlN} / \mathrm{SiO}_{2}$ and $\mathrm{Si}_{3} \mathrm{~N}_{4} / \mathrm{SiO}_{2} / \mathrm{Si}$ substrates were used for this purpose. For $\mathrm{AlN} / \mathrm{SiO}_{2}$, the original terminations on AlN likely suffered from the air-exposure and patterning; this increased the incubation time. Electron microscope and SE analyses suggested the formation of $\mathrm{GaN}$ and $\mathrm{Ga}$, on the $\mathrm{AlN}$ and $\mathrm{SiO}_{2}$ regions, respectively. For $\mathrm{Si}_{3} \mathrm{~N}_{4} / \mathrm{SiO}_{2} / \mathrm{Si}$, no noticeable difference in the thickness was observed on all three substrates. The AlN $/ \mathrm{Si}_{3} \mathrm{~N}_{4}$ substrate combination can be directly utilized for substrate-selective growth, because of the $\sim 2$ times longer incubation time on $\mathrm{Si}_{3} \mathrm{~N}_{4}$ compared to AlN. 


\section{References}

1. M. Biercuk, D. Monsma, C. Marcus, J. Becker and R. Gordon, Applied Physics Letters, 2003, 83, 2405-2407.

2. J. Goldberger, A. I. Hochbaum, R. Fan and P. Yang, Nano Letters, 2006, 6, 973-977.

3. A. Mackus and M. Merkx, https://www.atomiclimits.com/2019/07/18/fully-self-aligned-viasthe-killer-application-for-area-selective-ald-a-discussion-of-the-requirements-forimplementation-in-high-volume-manufacturing/, 2019.

4. H.-B.-R. Lee and S. F. Bent, Chemistry of Materials, 2011, 24, 279-286.

5. S. E. Atanasov, B. Kalanyan and G. N. Parsons, Journal of Vacuum Science \& Technology A: Vacuum, Surfaces, and Films, 2016, 34, 01A148.

6. M. M. Kerrigan, J. P. Klesko and C. H. Winter, Chemistry of Materials, 2017, 29, 74587466.

7. D. Zhuang and J. Edgar, Materials Science and Engineering: R: Reports, 2005, 48, 1-46. 


\section{Appendix 6.1: Wet etching of ALD (AI)GaN layers}

Wet etch tests of polycrystalline ALD (Al)GaN layers were performed in view of making structures to explore the substrate-selective aspect of the GaN ALD.

AlN layers deposited by thermal ALD, and GaN layers deposited by thermal- and PEALD were investigated. From the literature ${ }^{7}$, it was known that $\mathrm{H}_{3} \mathrm{PO}_{4}(85 \%$ by vol.) can etch polycrystalline AlN films at $60{ }^{\circ} \mathrm{C}$ and polycrystalline $\mathrm{GaN}$ films at $135^{\circ} \mathrm{C}$. However, the reported $(\mathrm{Al}) \mathrm{GaN}$ layers were not formed by ALD. Some other etchants include $\mathrm{NaOH}$, $\mathrm{KOH}$ and TMAH. Whereas $\mathrm{NaOH}$ or $\mathrm{KOH}$ can pose contamination problems in semiconductor devices, TMAH attacks standard cleanroom photoresists, therefore demanding a hard mask such as $\mathrm{Cr}$ or $\mathrm{SiO}_{2}$. Keeping these in mind, $\mathrm{H}_{3} \mathrm{PO}_{4}$ was used. A summary of the etch-rates of the $\mathrm{Al}(\mathrm{Ga}) \mathrm{N}$ layers and a photo of the realized $\mathrm{AlN}-\mathrm{on}-\mathrm{SiO}_{2}$ test-structures is shown in Fig. A 6.1 a and b, respectively. The extremely low etch rates of the GaN layers compared to the AlN at $60{ }^{\circ} \mathrm{C}$ may in future be beneficial for selective etching of AlN for specific device designs.

\begin{tabular}{|l|c|c|}
\hline \multicolumn{2}{|l|}{ Etch-rates from $85 \% \mathrm{H}_{3} \mathrm{PO}_{4}(\mathrm{~nm} / \mathrm{min})$} \\
\hline & at $60{ }^{\circ} \mathrm{C}$ & at $100{ }^{\circ} \mathrm{C}$ \\
\hline Thermal ALD AlN & 3.01 & na \\
\hline Thermal ALD GaN & 0.09 & 0.34 \\
\hline PEALD GaN & 0.29 & 4.57 \\
\hline
\end{tabular}

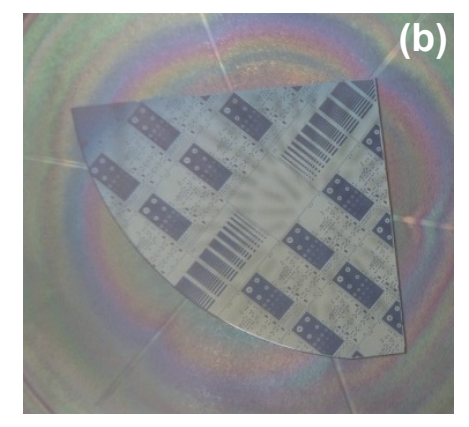

Fig. A 6.1 (a) Summary of the etch-rates of AlN and GaN layers by $85 \%$ by vol. $\mathrm{H}_{3} \mathrm{PO}_{4}$. (b) Patterned AlN-on- $\mathrm{SiO}_{2}$ test structures. 


\section{Appendix 6.2: Scanning electron microscope images of GaN ALD performed on} the ex-situ patterned $\mathrm{AlN} / \mathrm{SiO}_{2}$ substrate
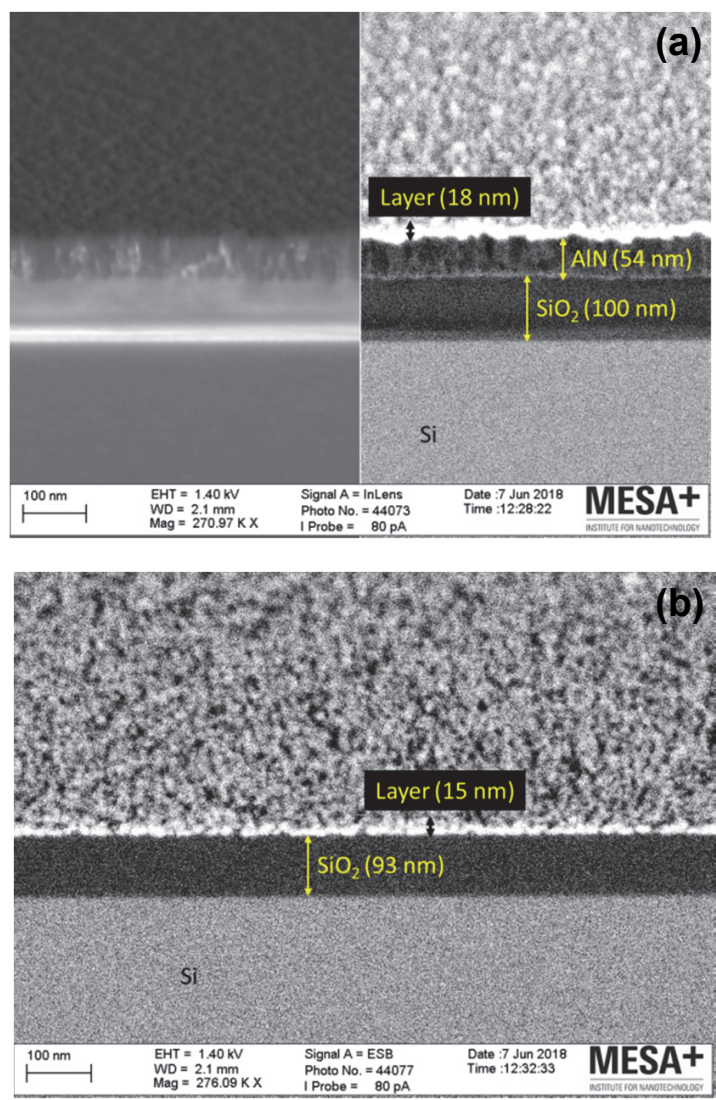

Fig. A 6.2 InLens SEM and ESB images of an ALD layer on (a) AIN and (b) $\mathrm{SiO}_{2}$ substrates. (Due to its unknown composition, the deposited layer is not named.) 


\section{Thermal ALD of AlN and} estimation of layer coalescence* $^{*}$

${ }^{*}$ This chapter is an extended version of:

S. Banerjee, R. van der Velde, M. Yang, J. Schmitz and A. Y. Kovalgin, ICMTS Conf., Grenoble, March 2017.

The fabrication of test-structures and electrical measurements were performed by $R$. van der Velde (Masters student) under the supervision of the author. 


\subsection{Introduction}

Although in ALD the layer growth occurs through a series of self-saturating surface reactions $^{1}$, this does not necessarily imply that a layer-by-layer, two-dimensional type of growth always occurs. This is because, the initial layer growth strongly depends on interactions between the substrate and the precursor species. Consequently, a coalesced layer ${ }^{*}$ may not form on the substrate from the beginning of the ALD, even though saturating precursor doses are supplied. Therefore, even though ALD is appreciated by many as the technique to deposit ultra-thin (e.g., sub-10 or sub-5 nm) layers, the formation of coalesced layers from the very first monolayers is a misconception. This is because ALD relies on surface chemistry. In the initial stages of the ALD, the chemical interaction between the terminations present on the substrate and the ligands of the precursor determine when the layer will become continuous. Prior to the coalescence, the layer nucleates at various locations on the substrate, and exists as discrete islands. The layer coalescence occurs after the formation of a few nanometers of such islands. In view of modern electron devices that demand such ultra-thin layers, it must therefore be verified whether the ALD chemistry used, can actually result in layer coalescence at such thicknesses. In this chapter, AlN deposited by thermal ALD from trimethylaluminum (TMA) and ammonia $\left(\mathrm{NH}_{3}\right)$ precursors, is used as a test case to check the coalescence thickness.

Section 7.2 discusses the different types of initial growth in ALD, showing which result and which do not result in a coalesced layer in the early ALD stages. Section $\mathbf{7 . 3}$ introduces thermal ALD of AIN and presents its material properties. Section 7.4 describes the use of a Metal-Insulator-Metal (M-I-M) structure to test the coalescence of AlN layers. Section 7.5 verifies the coalescence thickness by in-situ spectroscopic ellipsometry (SE), and Section 7.6 shows the pre-coalesced layer by high-resolution transmission electron microscope (HR-TEM) imaging. Section 7.7 concludes the chapter.

\subsection{Why ALD does not necessarily imply a coalesced layer}

\subsubsection{Types of initial growth in ALD processes}

During the deposition of thin layers, depending on the differences in the surface energy of the substrate and the layer to be deposited, the layer either wets or de-wets the substrate $^{2}$. Whereas the first case leads to a coalesced layer already in the initial growth stages, the latter leads to the formation of three-dimensional islands or 'clusters' of the layer on the substrate. Because ALD relies on surface chemistry, the specific chemistry of the

\footnotetext{
* In this chapter, the term 'coalesced' refers to a layer that has completely covered the substrate. The terms 'continuous' and 'closed' have also been used interchangeably.
} 
ALD process (i.e., as mentioned before, the nature of interaction between the terminations present on the substrate and the ligands present in the precursor) determine when the layer will coalesce.

The growth per cycle (GPC) can provide information on the nature of the initial growth of the layer ${ }^{3}$. During this stage the GPC is hardly constant, but is constantly changing due to the influence of the substrate, even when an optimized-for-ALD recipe is used. To quote Puurunen et al. ${ }^{3}$, "a constant GPC has been a paradigm in ALD through decades. Recently it has been realized, however, that because the ALD process changes the characteristics of the substrate through depositing new material, the GPC need not be constant. The effect of substrate on the GPC is seen in the beginning of the growth, when the substrate is not yet fully covered by the ALD-grown film. With increasing extent of deposition, the effect of the original substrate is lost and the GPC is expected to become constant". Accordingly, the authors present four types of GPC variation (Fig. 7.1). Fig. 7.1 a, representing a constant GPC from the very beginning, and typically resulting in a coalesced layer from the first cycle, is of course the ideal ALD process. However, it is observed only in few precursor and substrate combinations, such as chromia $\left(\mathrm{CrO}_{\mathrm{x}}\right)$ deposition on $\mathrm{Al}_{2} \mathrm{O}_{3}$, from $\mathrm{Cr}(\mathrm{acac})_{3}$ and $\mathrm{O}_{2}$ precursors ${ }^{4}$. A substrateenhanced growth (Fig. 7.1 b), i.e., when the GPC is higher on the substrate compared to that on the film itself, has also been observed in select thermal ALD processes such as that of cobalt $(\mathrm{Co})$ on $\mathrm{SiO}_{2}$ from $\mathrm{Co}(\mathrm{acac})_{3}$ and $\mathrm{O}_{2}$ precursors ${ }^{5}$, and also in several plasmaenhanced (PE-) ALD processes, such as that of $\mathrm{Ti}$ on $\mathrm{Pt}$ substrate, from $\mathrm{TiCl}_{4}$ and $\mathrm{H}_{2}$-plasma precursors ${ }^{6}$, and $\mathrm{AlN}$ and $\mathrm{GaN}$ layers on $\mathrm{Si}$ and $\mathrm{SiO}_{2}$ substrates, as observed in this work. In many thermal ALD processes, the variation is either type c or d, referred to as 'substrate-inhibited' ALD, which is discussed next.
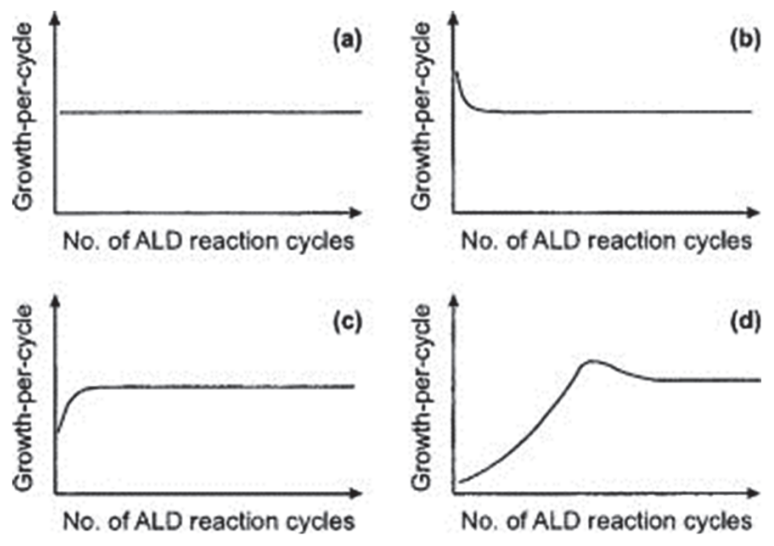

Fig. 7.1 Types of GPC evolution in ALD: (a) constant GPC (linear growth), (b) initially-high GPC (substrate-enhanced growth), (c, d) initially-low GPC (substrate-inhibited growth). (Original source: reference ${ }^{3}$ ) 


\subsubsection{Substrate-inhibited ALD}

The term 'substrate-inhibited' obviously implies that the substrate is not highly reactive to the ALD precursors. This can occur when, due to the terminations present, it is energetically unfavourable for the precursors to chemisorb ${ }^{7}$. The initial film nucleation can start through several ways depending on the specific chemistry of the ALD process. For instance, in the ALD of oxides (e.g., $\mathrm{ZrO}_{2}, \mathrm{Al}_{2} \mathrm{O}_{3}$ ) on H-terminated $\mathrm{Si}$ (the latter considered to be unfavourable towards the chemisorption of the respective precursors, $\mathrm{ZrCl}_{4}$ and TMA), the reactive defect surface sites such as $\mathrm{Si}-\mathrm{OH}$ and $\mathrm{Si}-\mathrm{O}-\mathrm{Si}$ are responsible for the initial precursor chemisorption ${ }^{8}$. In the ALD of nitrides, GaN growth possibly starts from isolated, TMG-chemisorbed surface sites on H-terminated $\mathrm{Si}$ and $\mathrm{OH}$-terminated $\mathrm{SiO}_{2}$ substrates, as addressed in the previous chapter. Substrate-inhibited ALD results in island-like (i.e., threedimensional or 3D) growth, forming isolated clusters of the film, typically of a few nanometers dimension. Such clusters during the initial growth of $\mathrm{Al}_{2} \mathrm{O}_{3}$ on $\mathrm{SiO}_{2}$ are reported $^{8}$. Similarly, we shall present pre-coalesced AIN clusters deposited on a PtSi layer by thermal ALD, later in this chapter (Section 7.6). Some other ALD processes that are reported to have a $3 \mathrm{D}$ mode of initial growth are TiN deposited on $\mathrm{SiO}_{2}{ }^{9}, \mathrm{SiO}_{2}$ deposited on $\mathrm{BN}^{10}$, and $\mathrm{WC}_{0.7} \mathrm{~N}_{0.3}$ deposited on polymer substrates ${ }^{11}$.

On continuing the ALD, the clusters grow in size laterally and vertically, due to the chemisorption of the precursors on already-formed clusters and on the exposed areas of the substrate, finally coalescing after several ALD cycles. The further film formation then occurs with a constant growth per cycle.

Besides the effect of the substrate terminations, other factors that may contribute to the $3 \mathrm{D}$ growth are (i) steric hindrance posed by the chemisorbed to the chemisorbing precursor, therefore blocking the neighbouring surface sites and preventing the chemisorption, (ii) desorption of the precursor back to the gas-phase prior to chemisorbing, and (iii) partial re-evaporation of the precursors or the deposited layer during purging ${ }^{12-14}$. These prevent the ideal monolayer-by-monolayer film formation even on supplying presumably saturating precursor doses. To quote Puurunen et al. again, "two-dimensional growth should not self-evidently be expected in ALD growth, however, especially in

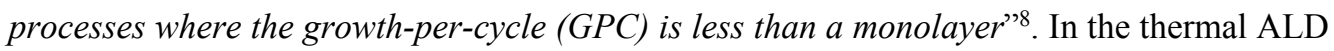
of $\mathrm{GaN}$, the strong dependence on the $\mathrm{NH}_{3}$ partial pressure may make factor (ii) an important candidate. In the thermal ALD of AIN, the pressure dependence is lower; but factor (i) or several others ${ }^{15}$ may disrupt the adduct-to-linkage conversion spatially, and therefore contribute to the $3 \mathrm{D}$ growth. 


\subsubsection{Tests for the substrate-inhibited ALD of AIN on PtSi: A brief overview}

The substrate-inhibited growth of AlN is expected to lead to a non-coalesced film in the initial stages. We have verified this and determined its coalescence-thickness using techniques, that rely on different physical phenomena: (i) with a M-I-M test-structure, and (ii) with in-situ SE monitoring of the film growth and observing the changes in GPC at different stages of ALD. Finally, using electron microscopy, the film at its pre-coalesced state is observed. These will be briefly explained below, before addressing them individually in detail.

7.2.3.1 With M-I-M structures: Since AlN is a dielectric, a M-I-M test-structure where AlN is sandwiched between two electrodes, is ideal to test its coalescence-thickness. Prior to the coalescence, the two electrodes contact each other, leading to ohmic vertical conduction. However, after AlN coalesces, the contact between the electrodes is disrupted and, due to the highly resistive nature of AlN, the magnitude of current drops significantly. In addition, the conduction changes from ohmic to non-ohmic type, suiting the dielectric layer. Although a M-I-M structure has been previously reported to observe the conduction mechanisms in ALD AlN films ${ }^{16}$, the estimation of its coalescence thickness or the idea that these structures are capable of assessing the coalescence of a dielectric thin film by ALD has not been reported to the best of our knowledge.

M-I-M structures, although simple to fabricate and measure, suffer from the drawback that on the application of a high electric field to a dielectric layer that has just coalesced, the electron flow can physically transform the dielectric into forming new conduction channels, some of which may be ohmic (e.g., as shown to occur in $\mathrm{SiO}_{2}{ }^{17}$ ). This may give the false impression that the film is yet in pre-coalescence. Also, the material of the electrodes may diffuse through the dielectric and contact each other, causing ohmic conduction. Therefore, these issues make determining the coalescence thickness not so straightforward. Supporting techniques, if available, must be used.

7.2.3.2 With in-situ $\boldsymbol{S E}$ : In-situ SE monitoring the film growth in real time can provide an estimate of the coalescence thickness. Since continuous monitoring is performed, the thickness at which the GPC stabilizes, presumably indicates coalescence. The in-situ approach provides this information in a single growth run, unlike the ex-situ techniques.

7.2.3.3 With electron microscopy: Electron-based imaging techniques such as HR-TEM, in combination with energy-dispersive X-ray spectroscopy (EDX) and energy-filtered (EF-) TEM, has been used in this work to verify that at sub-10 nm thicknesses, AlN exists mostly in the form of isolated clusters. 


\subsection{Thermal ALD of AIN and material properties}

The thermal ALD of AlN was performed using TMA and $\mathrm{NH}_{3}$ precursors at $350^{\circ} \mathrm{C}$, on $\mathrm{Si}(111)$ substrates. The absence of carbon in the layers (Fig. $7.3 \mathrm{~b}$, showing the depthprofiled composition) suggests that this temperature is below the dissociation threshold of TMA. Fig. 7.2 a shows the variation of GPC with the deposition temperature. At $300{ }^{\circ} \mathrm{C}$ the GPC is almost negligible, whereas beyond $350{ }^{\circ} \mathrm{C}$ the high GPC values reflect the possible departure from the self-limiting mechanism, due to the dissociation of $\mathrm{TMA}^{18}$.

Detailed ALD 'window-search' experiments were not done for this particular ALD process: the durations and the flow rates of the precursors and purges were copied from our prior developed recipe for $\mathrm{AlN}^{19}$. However, the deposition parameters were still optimized to achieve the best uniformity on a 4-inch Si substrate (Fig. 7.2 b). The optimized recipe (in caption) yielded decent conformality on Si trenches (Fig. 7.2 c). Thickness measurements performed at various locations on the trenches yielded an average of $38 \mathrm{~nm}$ and standard deviation of $3 \mathrm{~nm}$.
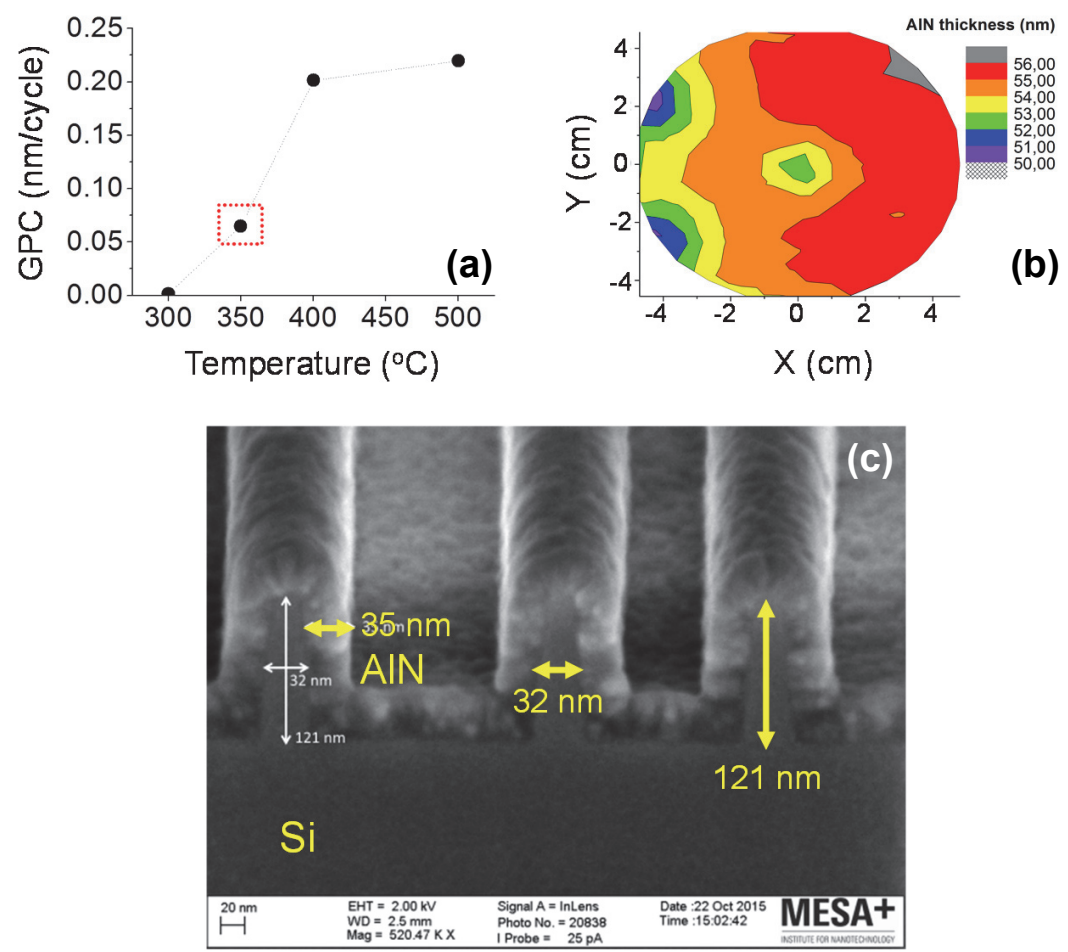

Fig. 7.2 (a) Variation of GPC with ALD temperature. The ALD recipe was: $0.1 \mathrm{~s}$ TMA / $4 \mathrm{~s} \mathrm{Ar} / 2 \mathrm{~s}$ $\mathrm{NH}_{3} / 4 \mathrm{~s}$ Ar, performed at $10^{-2}$ mbar reactor pressure. The red box $\left(350{ }^{\circ} \mathrm{C}\right)$ shows the temperature used for subsequent depositions. (b) Thickness variation of AIN on a 4-inch Si wafer (6\% non-uniformity) obtained with the optimized recipe. (c) Conformal AlN layer deposited on Si trenches. 
A Cauchy-Urbach optical model in the SE was used to determine the optical constants of AlN (Fig. 7.3 a). The obtained refractive index of 1.97 (at $632.8 \mathrm{~nm}$ ) is in good agreement with that (2.0), reported for ALD AlN layers elsewhere ${ }^{20}$. The layer also exhibited a high transparency beyond $600 \mathrm{~nm}$, as evident from the negligible $k$ (Fig. 7.3 a). The SE measurements were performed on a thick AlN layer $(100 \mathrm{~nm})$, after the optical constants stabilized ${ }^{\dagger}$.

Sputter depth profiled XPS analysis revealed a near-stoichiometric AlN layer (Fig. 7.3 b) with negligible carbon content. The bulk of the layer also contained negligible oxygen, but the surface was oxidized from transport to the XPS instrument. The polycrystalline structure of the layer was revealed from the GIXRD scan (Fig. 7.3 c). The peaks and their Miller indices correspond to wurtzitic AIN (COD No. 9008860).
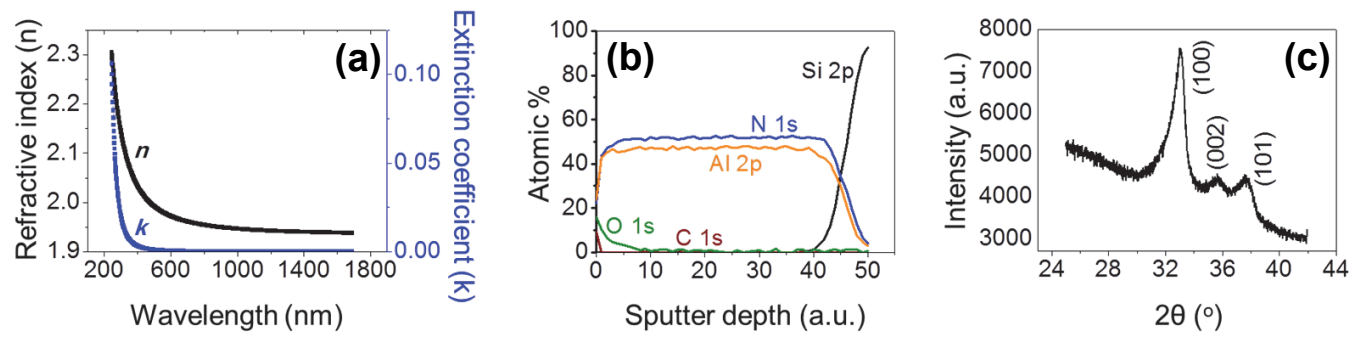

Fig. 7.3: (a) Variation of AIN optical constants $(n, k)$ with wavelength. (b) XPS depth profiled composition revealing a near-stoichiometric AIN with low $\mathrm{O}$ and $\mathrm{C}$ impurities. (c) GIXRD scan showing polycrystalline wurtzitic structure.

\subsection{Test for AIN coalescence (with M-I-M structures)}

\subsubsection{M-I-M fabrication}

The schematic of the M-I-M test structure is shown in Fig. 7.4 a. The substrate was a p-type $\mathrm{Si}$ wafer on which $100 \mathrm{~nm} \mathrm{SiO} 2$ was thermally grown. A $5 \mathrm{~nm}$ Ti adhesion layer was sputtered, followed by sputtering $30 \mathrm{~nm} \mathrm{Pt}$. The Ti/Pt stack was patterned by lift-off, resulting in well-defined contact pads serving as the bottom electrode. Subsequently, a $7 \mathrm{~nm}$ CVD amorphous Si (a-Si) layer was deposited by LPCVD at $350{ }^{\circ} \mathrm{C}$. ALD AIN was then deposited without vacuum break in the same reactor, at $350{ }^{\circ} \mathrm{C}$, with thicknesses ranging from 4 to $35 \mathrm{~nm}$ for different samples. The a-Si serves as an adhesion layer, as AlN

\footnotetext{
$\dagger$ The optical constants of AIN are reported to stabilize well beyond the coalescence thickness, due to the still-changing layer properties such as density and morphology ${ }^{19}$. This thickness is between $35-40 \mathrm{~nm}$ of $\mathrm{AlN}$ deposited on $\mathrm{Si}$ and $\mathrm{SiO}_{2}$ substrates.
} 
deposited directly on Pt was observed to peel-off during subsequent processing. A second a-Si layer $(14 \mathrm{~nm})$ was in-situ deposited at the same temperature (i.e., at $350{ }^{\circ} \mathrm{C}$ ), as a capping layer on AIN, to prevent oxidation of the latter during the transport of the wafer for the top-electrode deposition. A $50 \mathrm{~nm}$ sputtered Pt layer served as the top electrode, which was also patterned by lift-off.

The a-Si itself is highly resistive. However, prior to and after the AIN ALD, the test-structure was annealed at $200{ }^{\circ} \mathrm{C}\left(\mathrm{N}_{2}\right.$ ambient, $\left.25 \mathrm{~min}, 10 \mathrm{mbar}\right)$ to cause silicidation between Pt and a-Si. The a-Si layer was consumed and formed a conducting PtSi layer on the electrodes $^{21-22}$. These process steps finally resulted in a $\mathrm{Si} / \mathrm{Ti} / \mathrm{Pt} / \mathrm{PtSi} / \mathrm{AlN} / \mathrm{PtSi} / \mathrm{Pt}$ stack. Measurements were done with two probes (shown by arrows in Fig. 7.4 a). Placing the probes directly on the stack or the 'region under test' were avoided to prevent damage to the AlN. A three-dimensional layout can be found in Appendix 7, Fig. A 7.1.
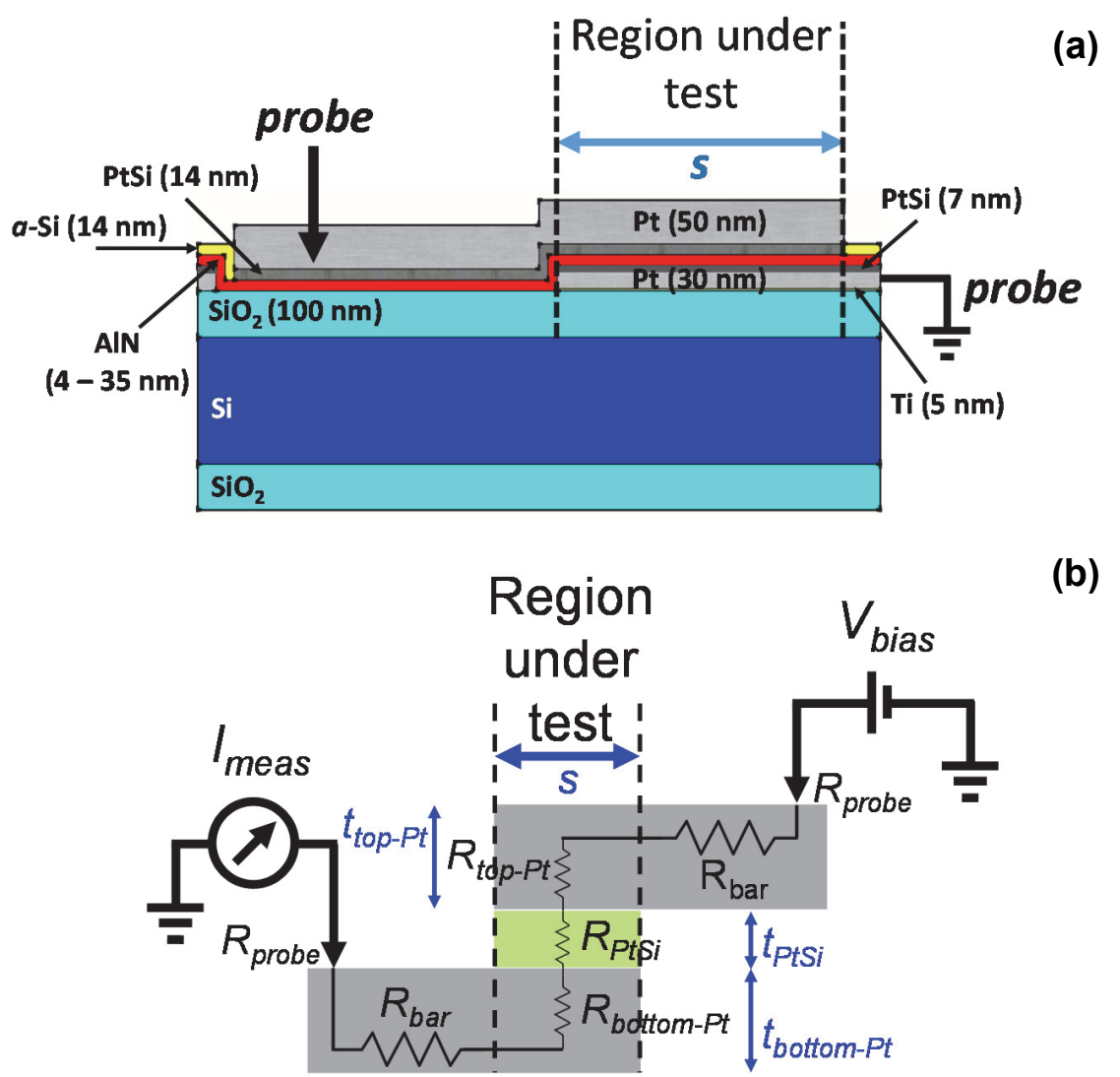

(b)

Fig. 7.4: (a) The side-view schematic of the M-I-M test structure (the dimensions are not to scale). (b) The equivalent electrical circuit of the structure without an AlN layer between the PtSi layers (in green). 


\subsubsection{M-I-M without AIN layer}

A test-structure without an AIN layer has the top and bottom electrodes in physical contact. The equivalent circuit of this structure is shown in Fig. 7.4 b. The measuring probes are placed on the two extensions or 'bars'. Different structures, with different sizes $(s)$ of the so-called 'region under test' (i.e., the contact areas sandwiched between the two square Pt electrodes, sides $s \times s$ ), were fabricated. Each bar had an area (also $s \times s$ ) and thickness as that of the top (or bottom) Pt electrodes.

The overall resistance ( $R_{\text {tot }}$ ) of the test structure is defined as $V_{\text {bias }} / I_{\text {meas, }}$ where $V_{\text {bias }}$ is the applied bias at one of the bars and $I_{\text {meas }}$ is the measured current at the other bar. $R_{\text {tot }}$ is the sum of two components: the resistance of the region-under-test $\left(R_{\text {stack }}\right)$ and additional resistances lumped as $R_{\text {ext. }} R_{\text {ext }}$ includes the resistance of the bars $\left(R_{\text {bar }}\right)$ and the probe resistance $\left(R_{\text {probe }}\right)$. Plugging in the generally-accepted literature resistivity values of $\mathrm{Pt}$ and PtSi yields $R_{\text {stack }}$ of $10^{-14} / s^{2}$ (in ohm). This implies that $R_{\text {stack }}$ is negligible without an AlN layer in the region-under-test, and therefore, $R_{\text {tot }}$ is governed by $R_{\text {ext. }}$ Consequently, $I_{\text {meas }}$ equals $V_{\text {bias }} / R_{\text {ext }}$ and becomes a constant irrespective of the stack area $\left(s^{2}\right)$.

The variation of $I_{\text {meas }}$ with $V_{\text {bias }}$ at four different stack areas (i.e., $s^{2}$ ) supports the above discussion (Fig. 7.5 a). $I_{\text {meas }}$ varies linearly with $V_{\text {bias }}$ and indeed shows no consistent

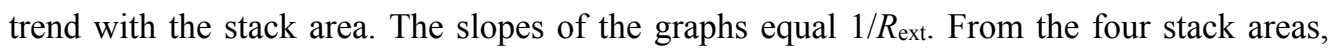
$R_{\text {ext }}$ is estimated as $13.2 \pm 1.6 \Omega$. The validity of the estimation is verified by plugging in the value of $R_{\text {bar }}$ as $3.17 \Omega$ (obtained from the known resistivity of $\mathrm{Pt}$ and the thicknesses of the top and bottom electrodes) and $R_{\text {probe }}$ of $2.85 \Omega$ (obtained from separate 2-probe measurements). This yields $R_{\text {ext }}$ of $12 \Omega$, which is in fair agreement with the estimated value. An ohmic conduction also implies the proper silicidation of the electrodes.

\subsubsection{M-I-M with AIN layers of various thicknesses}

7.4.3.1 AlN in the pre-coalesced state: A test-structure with a $4 \mathrm{~nm}$ thick AlN layer (the thickness was obtained from in-situ SE) was characterized. The corresponding $I_{\text {meas }}$ vs. $V_{\text {bias }}$ plots are ohmic (Fig. 7.5 b). Similar to Fig. 7.5 a, no consistent trend of $I_{\text {meas }}$ with the stack areas is obtained, and the estimated $R_{\text {ext }}$ is similar to the previous. This implies that at $4 \mathrm{~nm}$, the AlN does not exist as a continuous layer but rather as clusters, which allows the electrodes to contact one another ${ }^{\ddagger}$. The equivalent electrical circuit for this case is shown in Appendix 7, Fig. A 7.2.

$\$$ To note: Ohmic conduction in (presumably-closed) $7 \mathrm{~nm}$ AlN layers have been reported at low (up to $1.5 \mathrm{~V}$ ) voltages ${ }^{26}$. However, the current densities reported there are several orders of magnitude lower 
The discontinuity of the AlN film at $4 \mathrm{~nm}$ is further confirmed by a control experiment. An almost linear shape of $I_{\text {meas }}$ vs. $V_{\text {bias }}$ that is independent of the stack area, is obtained even without the silicidation of the electrodes (Fig. 7.5 c). An explanation is that, the top electrode underwent silicidation during the a-Si deposition on the AlN layer. Such could have happened if the AlN was discontinuous; the a-Si contacted the bottom electrode, siliciding itself at the deposition temperature of $350{ }^{\circ} \mathrm{C}$.
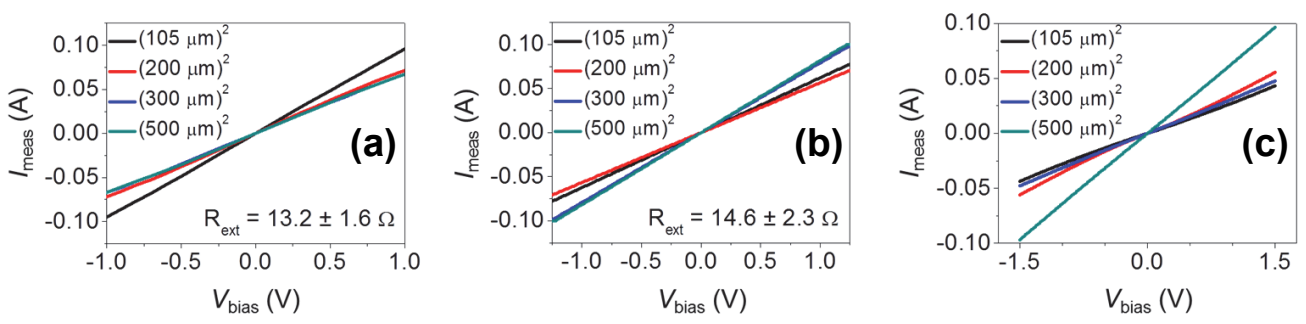

Fig. 7.5 $I_{\text {meas }}$ vs. $V_{\text {bias }}$ plots for test-structures of different stack areas: (a) without AlN, (b) with $4 \mathrm{~nm}$ AlN after annealing, and (c) with $4 \mathrm{~nm}$ AlN before annealing. $R_{\text {ext }}$ has been determined from the slopes of the plots in figures $\mathbf{a}$ and $\mathbf{b}$.

7.4.3.2 AlN in the coalesced state: A $35 \mathrm{~nm}$ AlN film between the electrodes can be expected, owing to the high thickness, to be fully coalesced and hence isolate the electrodes. The equivalent electrical circuit for this case is shown in Appendix 7, Fig. A 7.3. As the resistivity of $\mathrm{AlN}\left(\rho_{\mathrm{AlN}}\right)$ is orders of magnitude higher than both $\mathrm{Pt}$ and $\mathrm{PtSi}, R_{\text {stack }}$ is now dominated by the presence of the AlN layer. Consequently, $I_{\text {meas }}$ tends to $V_{\text {bias }} /\left(\rho_{\text {AlN }} t_{\text {AlN }} / s^{2}\right)$. $I_{\text {meas }}$ can therefore be expected to depend on the stack area.
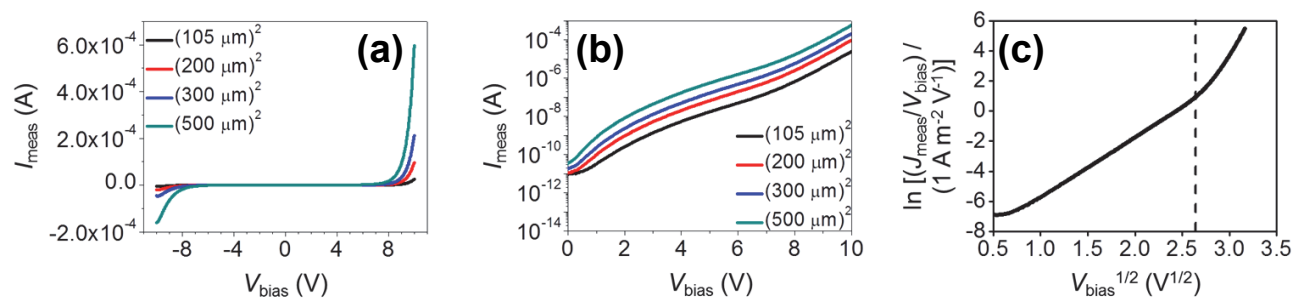

Fig. 7.6 (a) $I_{\text {meas }}$ vs. $V_{\text {bias }}$ plot of $35 \mathrm{~nm}$ AlN film. (b) The same plot visualized in the logarithmic scale to show the clear variation of $I_{\text {meas }}$ with the stack area. (c) Poole-Frenkel conduction exhibited up to $\sim 7 \mathrm{~V}$ by the film.

than what obtained in this work (e.g., $10^{-11}$ vs. $10^{2} \mathrm{~A} \mathrm{~cm}^{-2}$ ). This gives another hint that the AlN in our case is not a continuous layer. 
Fig. 7.6 a shows the $I_{\text {meas }}$ vs. $V_{\text {bias }}$ plots for the $35 \mathrm{~nm}$ AlN film. The following observations are made: (i) the current levels have now decreased by orders of magnitude compared to Fig. $7.5 \mathbf{b}$ and $\mathbf{c}$, (ii) the conduction has become highly non-ohmic, and (iii) $I_{\text {meas }}$ has started to show a consistent trend with the stack area. Fig. 7.6 b is a representation of Fig. 7.6 a on the logarithmic scale to better visualize the area dependency. From Fig. 7.6 b, $\rho_{\text {AlN }}$ was determined between $0-0.5 \mathrm{~V}$ to be $6.20 \pm 1.27 \times 10^{17} \Omega \mu \mathrm{m}$, which is consistent with that reported for AlN elsewhere ${ }^{23}$. Moreover, no systematic variation of $\rho_{\text {AlN }}$ with the stack area was observed. All these confirm the presence of a coalesced highly resistive AlN layer between the electrodes. A thinner $(15 \mathrm{~nm})$ layer also exhibited the same observations.

Several current transport phenomena (e.g., emission, tunnelling) in ALD AlN films of comparable thicknesses have been previously reported ${ }^{16}$. The dominant conduction mechanism for our AIN film is found to be Poole-Frenkel (PF) emission in the $V_{\text {bias }}$ range of $0.6-7 \mathrm{~V}$ from its linearity, when plotted according to the PF expression (Fig. 7.6 c). The conduction therefore changes from ohmic in uncoalesced AIN to PF-type in coalesced.

7.4.3.3 AlN in the transition state: An $11 \mathrm{~nm}$ AlN film, prior to siliciding the top electrode, exhibited highly non-ohmic conduction with an extremely low magnitude of $I_{\text {meas }}$ (Fig. 7.7 a). In fact, the current levels were far lower than those in Fig. 7.6 a. This suggests that the AIN layer was coalesced at this thickness, and the low current levels were due to the unsilicided a-Si layer on AIN.

However, post-annealing the top electrode results in a dramatic change of conduction. It becomes ohmic and $I_{\text {meas }}$ increases by several orders of magnitude (Fig. 7.7 b), matching that of the un-coalesced film (recall Fig. $7.5 \mathbf{b}$ or c). The change in the conduction pattern presumably occurs due to the diffusion, and eventually forming the contact, by the top and the bottom electrodes. The diffusion could occur through the thinnest regions of the just-coalesced AlN layer via grain boundaries or pin-holes, therefore establishing contact points between the electrodes. 

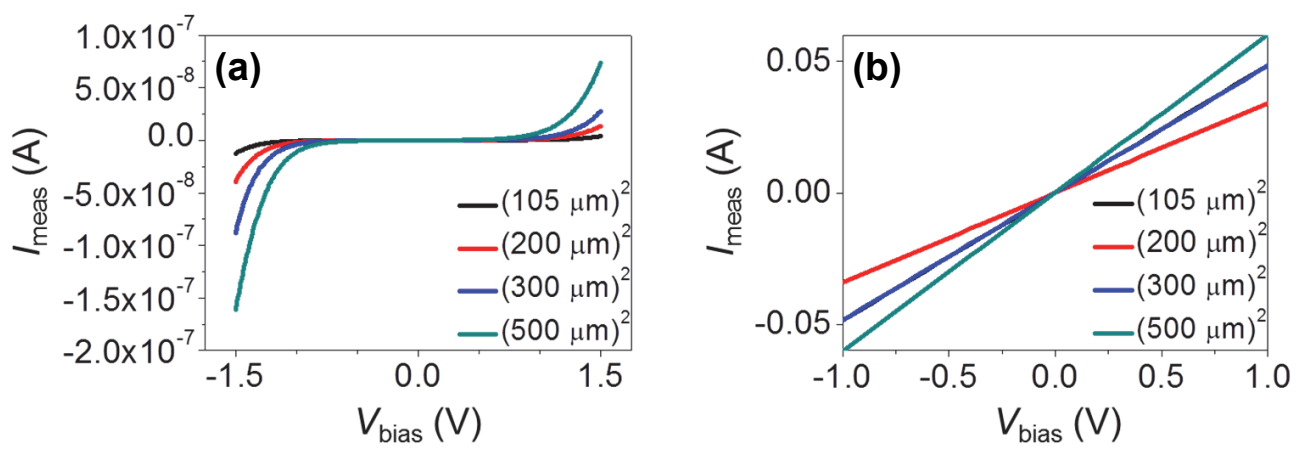

Fig. 7.7 $I_{\text {meas }}$ vs. $V_{\text {bias }}$ plot of $11 \mathrm{~nm}$ AlN film (a) before annealing, (b) after annealing.

\subsection{Test for AIN coalescence (with in-situ SE)}

As in-situ SE monitors the layer growth continuously, it can provide an estimate of the AlN coalescence in a single deposition run. The evolution of the AlN thickness and GPC with ALD cycles on PtSi is shown in Fig. 7.8 ${ }^{\S}$ The GPC increases monotonically during the initial ALD stages, and only after a certain thickness is reached, it stabilizes. The trend pertains to substrate-inhibited growth (recall Fig. 1 c, d). The various stages of growth are described below.

$0-125$ cycles: The delay in the commencement of growth, as also observed from the unchanging $\Psi$ and $\Delta$ values in the ellipsometric signal, represents the incubation stage. During this stage, the difficulty of chemisorption of TMA to the PtSi substrate perhaps results in isolated AIN clusters, and hardly any growth is observed.

125 - 300 cycles: With continued ALD, the so-formed clusters (with the remaining areas of the substrate) serve as the new chemisorption sites for TMA. The coverage of the clusters gradually increases, and the clusters serve as more favourable nucleation sites than the substrate itself, as observed from the steadily-increasing GPC. This phase ends when the AlN thickness reaches $\sim 5 \mathrm{~nm}$.

${ }^{\S}$ Commenting on the nature of the initial film growth by in-situ SE is not straightforward without a rigorous optical modelling procedure for the un-coalesced layer (see, for example, reference ${ }^{25}$ ). This was beyond the scope of this work. Instead, a Cauchy optical model was used, assuming AlN to be a continuous layer. However, by definition, this is contradictory since the layer is discontinuous initially. The subsequent discussion on the growth stages is therefore speculative and represents processes that might be occurring. 
300 - 365 cycles: The monotonic increase stops and the GPC starts showing a saturation tendency. This indicates the fast diminishing areas of the substrate, or, the onset of the layer coalescence.

Beyond 365 cycles: The GPC stabilizes, indicating a coalesced layer. This corresponds to $8.4 \mathrm{~nm}$ AlN. For the remaining ALD, the film grows with a constant GPC of $0.065 \mathrm{~nm} / \mathrm{cycle}$.

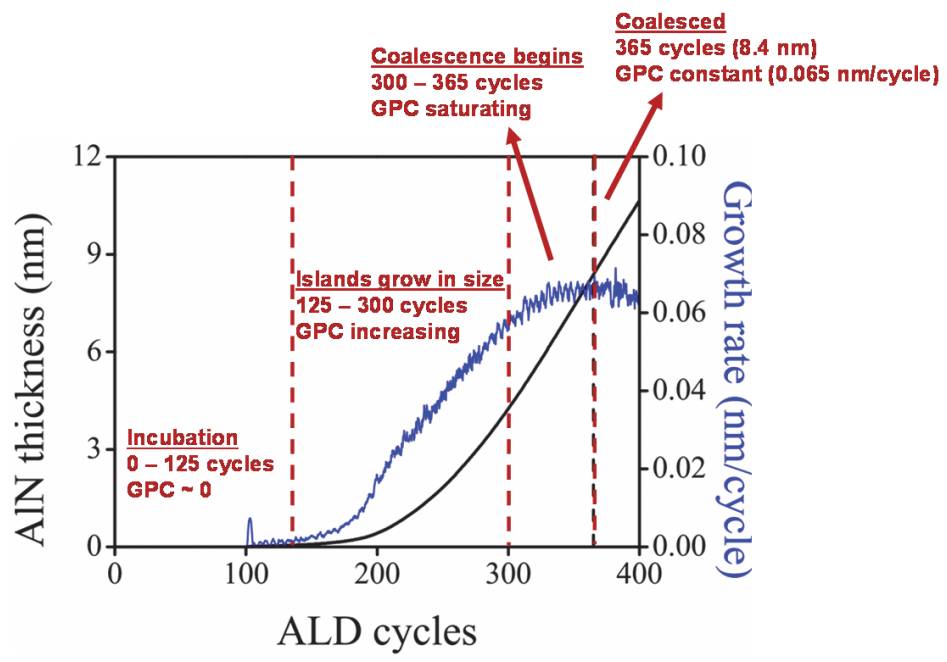

Fig. 7.8: Evolution of the AIN film thickness (in black) and GPC (in blue) with ALD cycles. The four regimes of growth are highlighted. The AlN fully coalesces at $8.4 \mathrm{~nm}$ corresponding to saturation of the GPC.

\subsection{Pre-coalesced AIN clusters observed with electron microscopy}

The pre-coalesced island-like state of the $4 \mathrm{~nm}$ AlN was observed by HR-TEM. Fig. 7.9 a depicts a cross-section HR-TEM image of the M-I-M structure, showing the electrode stack (the region of the TEM is shown in Appendix 7, Fig. A 7.4). The composition at regions 1-3 was verified by EDX (Fig. 7.9 b). Embedded in the PtSi layer, clusters having bead-like appearances can be observed in the HR-TEM image. Only Al is detected in region 1, from the location of the clusters. Due to the low sensitivity of the EDX towards $\mathrm{N}$, the latter is probably not detected. These clusters could therefore be the uncoalesced AlN layer or indeed just Al (in the event of the later, likely as oxide).

For a more conclusive analysis, HR-EELS imaging of the same region was performed, and EF-TEM elemental maps were constructed (an example of the latter is shown in Fig. 7.10). Si, Ti and $\mathrm{N}$ elements were mapped. Besides the expected elements at their corresponding regions ( $\mathrm{Si}, \mathrm{Ti}$ ), a clear continuous $\mathrm{N}$ signal (in red) appears from the PtSi 
layer (in blue). At the used magnification, this does appear to be a continuous N-containing layer. Therefore a higher magnification was used, as presented below.

Fig. 7.11 a - d shows several HR-TEM images from different locations inside the PtSi matrix. Numerous clusters, in their pre-coalescence state, can be seen embedded in the matrix. The dimensions of these clusters are sub-10 nm.

Fig. 7.12 a, b shows the EF-TEM elemental maps corresponding to Fig. 7.11 c, d, respectively. In Fig. 7.12 a, the blue band represents the PtSi matrix, and in Fig. 7.12 b, the yellow band represents the same. Nitrogen is shown by red and green in Fig. 7.12 a and b, respectively. The red band in Fig. 7.12 b represents glue. These high resolution EF-TEM images clearly show that the distribution of nitrogen is not continuous but rather clustered. The identification of not continuous, but rather discrete $\mathrm{N}$-rich regions in the PtSi matrix makes us conclude that these are the AlN clusters in their pre-coalesced state.
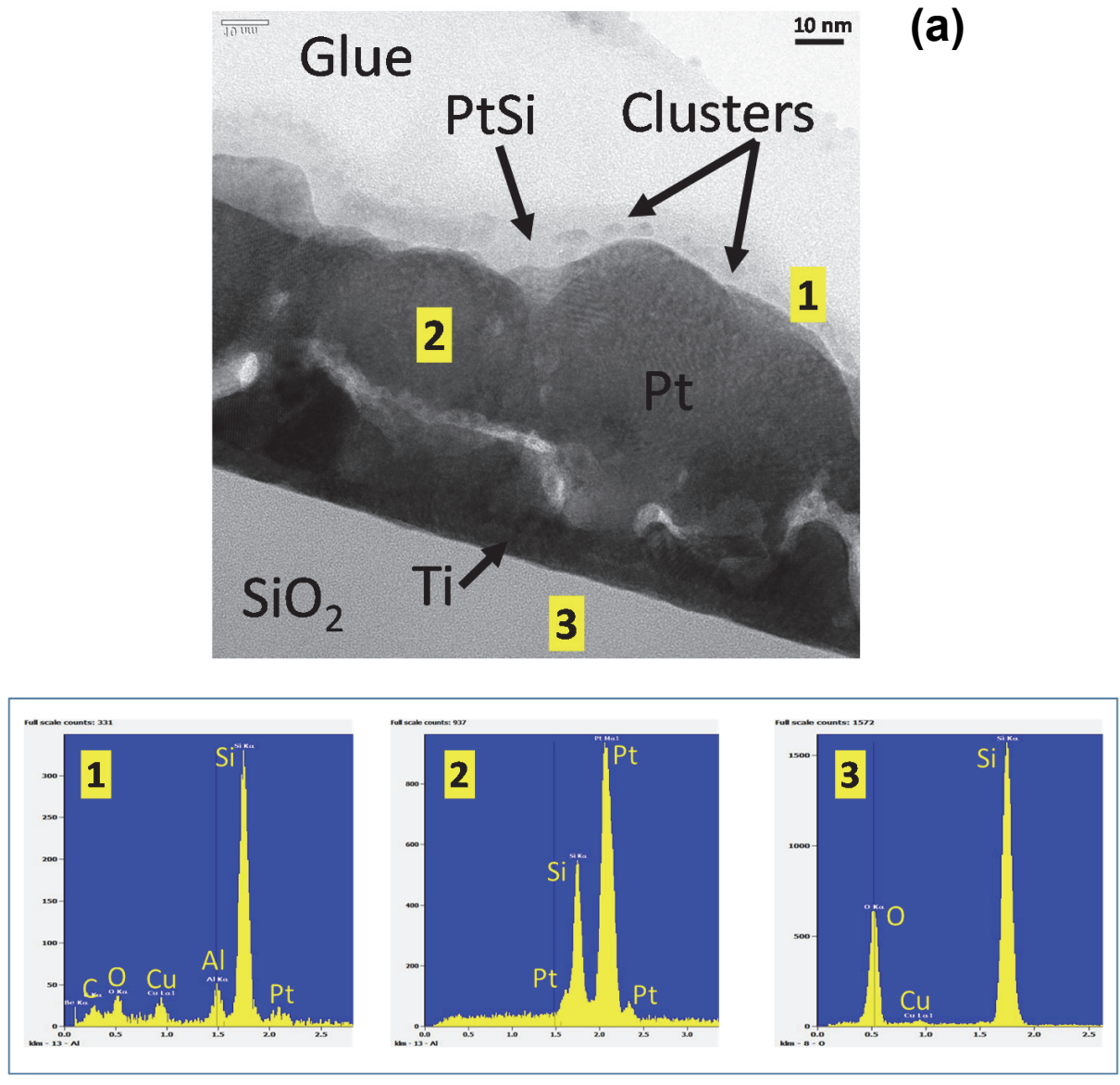

(b)

Fig. 7.9 (a) TEM and (b) EDX images of the electrode stack, corresponding to the regions $1-3$. 


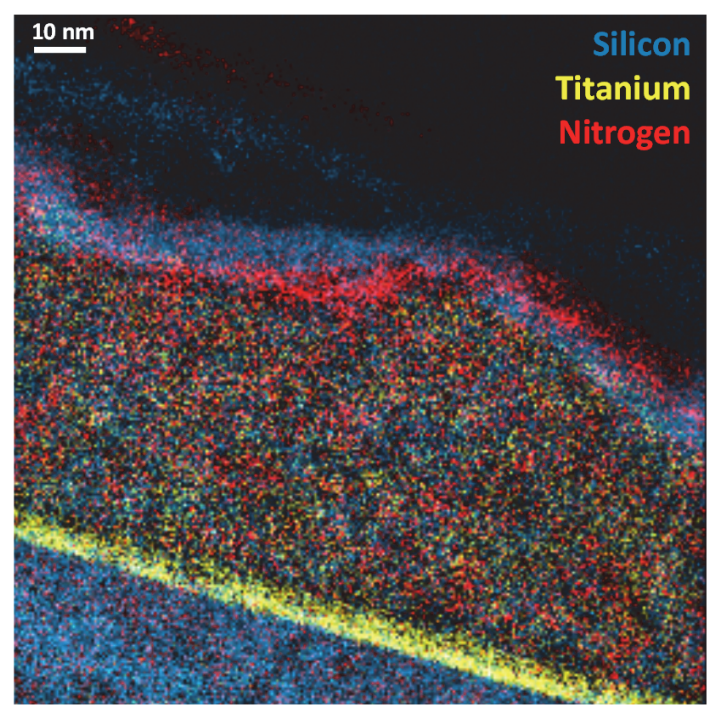

Fig. 7.10 EF-TEM elemental map corresponding to Fig. 7.9 a.
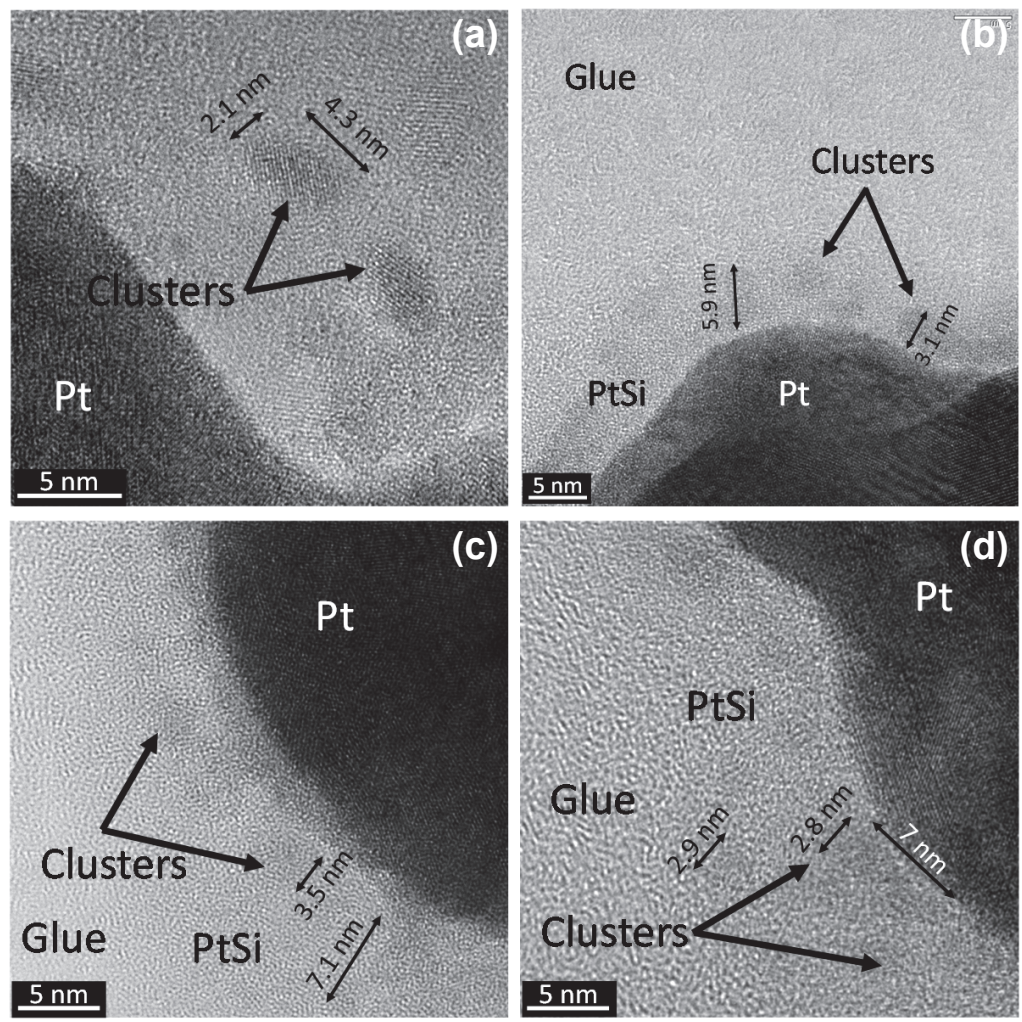

Fig. 7.11 (a - d) HR-TEM images from several regions in PtSi matrix, showing embedded clusters. 

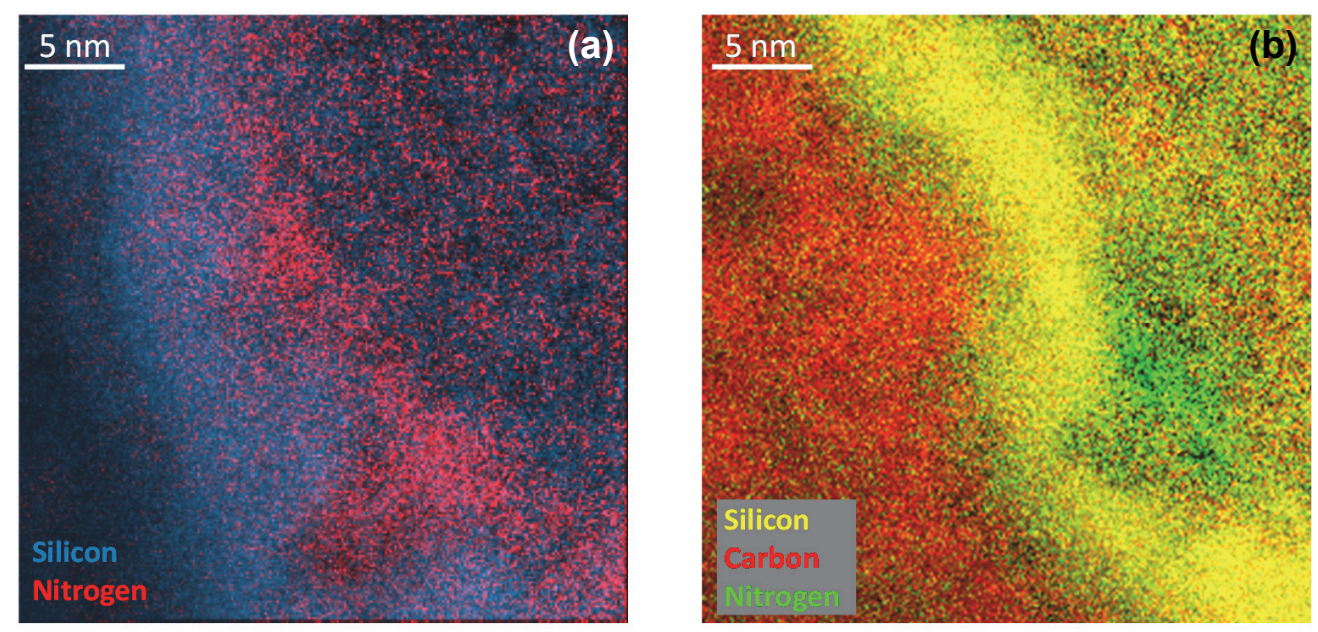

Fig. 7.12 (a, b) EF-TEM elemental maps corresponding to Fig. 7.11 c and d, respectively.

\subsection{Conclusions}

In this chapter, we addressed the causes of an un-coalesced layer formation in the initial stages of ALD, and investigated this for AIN layers prepared by thermal ALD. AIN, being a dielectric, offered the possibility of using M-I-M test structures to test the coalescence thickness. The latter was estimated to be slightly below $11 \mathrm{~nm}$. This was concluded from (i) a decrease in the current level up to three orders of magnitude, (ii) dependence of current on the stack area, and (iii) transition from ohmic to PooleFrenkel conduction, from the pre-coalescence to the post-coalescence stages.

The electrical analysis was supported by in-situ monitoring the AIN growth by SE. The layer coalescence was determined from the thickness at which the GPC stabilized, which was $8.4 \mathrm{~nm}$. This value is in fair agreement with the electrically determined estimate, considering the techniques rely on vastly different physical mechanisms ${ }^{24}$. The pre-coalesced state of the AIN (4 nm thickness by SE) was confirmed by HR-TEM and EF-TEM imaging, where the dimensions of the clusters ranged from 2 to $7 \mathrm{~nm}$. In conclusion, the carried out analyses revealed that the AlN layer coalesced at approximately $10 \mathrm{~nm}$. 


\section{References}

1. R. L. Puurunen, Journal of Applied Physics, 2005, 97, 9.

2. H. C. Knoops, S. E. Potts, A. A. Bol and W. Kessels, in Handbook of Crystal Growth, Elsevier, 2015, 1101-1134.

3. R. L. Puurunen and W. Vandervorst, Journal of Applied Physics, 2004, 96, 7686-7695.

4. A. Kytökivi, J.-P. Jacobs, A. Hakuli, J. Meriläinen and H. H. Brongersma, Journal of Catalysis, 1996, 162, 190-197.

5. A. Rautiainen, M. Lindblad, L. B. Backman and R. L. Puurunen, Physical Chemistry Chemical Physics, 2002, 4, 2466-2472.

6. H. Kim and S. Rossnagel, Journal of Vacuum Science \& Technology A: Vacuum, Surfaces, and Films, 2002, 20, 802-808.

7. J.-W. Lim, H.-S. Park and S.-W. Kang, Journal of The Electrochemical Society, 2001, 148, C403-C408.

8. R. L. Puurunen, W. Vandervorst, W. F. Besling, O. Richard, H. Bender, T. Conard, C. Zhao, A. Delabie, M. Caymax and S. De Gendt, Journal of Applied Physics, 2004, 96, 4878-4889.

9. A. Satta, J. Schuhmacher, C. Whelan, W. Vandervorst, S. Brongersma, G. Beyer, K. Maex, A. Vantomme, M. Viitanen and H. Brongersma, Journal of Applied Physics, 2002, 92, 76417646.

10. J. Ferguson, A. Weimer and S. George, Chemistry of Materials, 2000, 12, 3472-3480.

11. A. M. Hoyas, J. Schuhmacher, D. Shamiryan, J. Waeterloos, W. Besling, J.-P. Celis and K. Maex, Journal of Applied Physics, 2004, 95, 381-388.

12. H.-S. Park, J.-S. Min, J.-W. Lim and S.-W. Kang, Applied Surface Science, 2000, 158, 81-91.

13. S. George, A. Ott and J. Klaus, The Journal of Physical Chemistry, 1996, 100, 13121-13131.

14. M. Herman, M. Vulli and M. Pessa, Journal of Crystal Growth, 1985, 73, 403-406.

15. M. Bartram, T. Michalske, J. Rogers Jr and R. Paine, Chemistry of Materials, 1993, 5, 14241430 .

16. H. Altuntas, C. Ozgit-Akgun, I. Donmez and N. Biyikli, Journal of Applied Physics, 2015, 117, 155101.

17. V. E. Houtsma, Gate Oxide Reliability of Poly-Si and Poly-SiGe CMOS Devices, Ph.D Thesis, University of Twente, 1999.

18. N. Suzuki, C. Anayama, K. Masu, K. Tsubouchi and N. Mikoshiba, Japanese Journal of Applied Physics, 1986, 25, 1236. 
19. H. Van Bui, M. Nguyen, F. B. Wiggers, A. A. I. Aarnink, M. P. de Jong and A. Y. Kovalgin, ECS Journal of Solid State Science and Technology, 2014, 3, P101-P106.

20. E. Goldenberg, C. Ozgit-Akgun, N. Biyikli and A. Kemal Okyay, Journal of Vacuum Science \& Technology A: Vacuum, Surfaces, and Films, 2014, 32, 031508.

21. E. J. Faber, R. A. M. Wolters and J. Schmitz, IEEE Transactions on Semiconductor Manufacturing, 2012, 25, 339-345.

22. H. Van Bui, F. B. Wiggers, M. P. de Jong and A. Y. Kovalgin, ICMTS Conf., Udine, March 2014.

23. J. Edwards, K. Kawabe, G. Stevens and R. Tredgold, Solid State Communications, 1965, 3, 99-100.

24. H. Van Bui, A. Y. Kovalgin and R. A. M. Wolters, Applied Surface Science, 2013, 269, 45-49.

25. H. Wormeester, E. S. Kooij and B. Poelsema, Physica Status Solidi (A), 2008, 205, 756-763.

26. H. Altuntas, C. Ozgit-Akgun, I. Donmez and N. Biyikli, IEEE Transactions on Electron Devices, 2015, 62, 3627. 
Appendix 7: Schematics and equivalent electrical circuits of the M-I-M test structure before and after coalescence

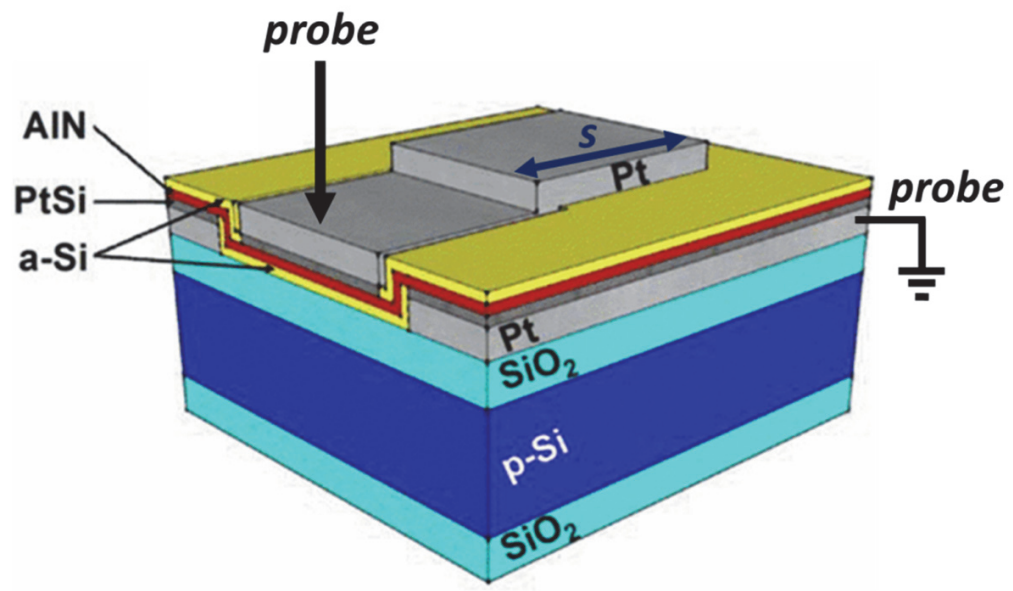

Fig. A 7.1 Three-dimensional layout of the test-structure. The layer thicknesses are the same as that of Fig.7.4 a.

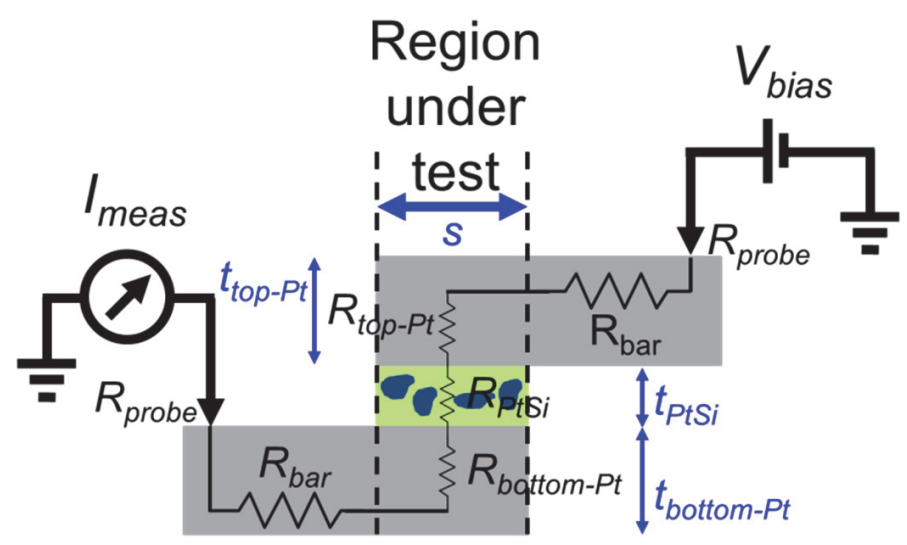

Fig. A 7.2 Equivalent electrical circuit of the test structure with a pre-coalesced AlN layer (dark blue). 


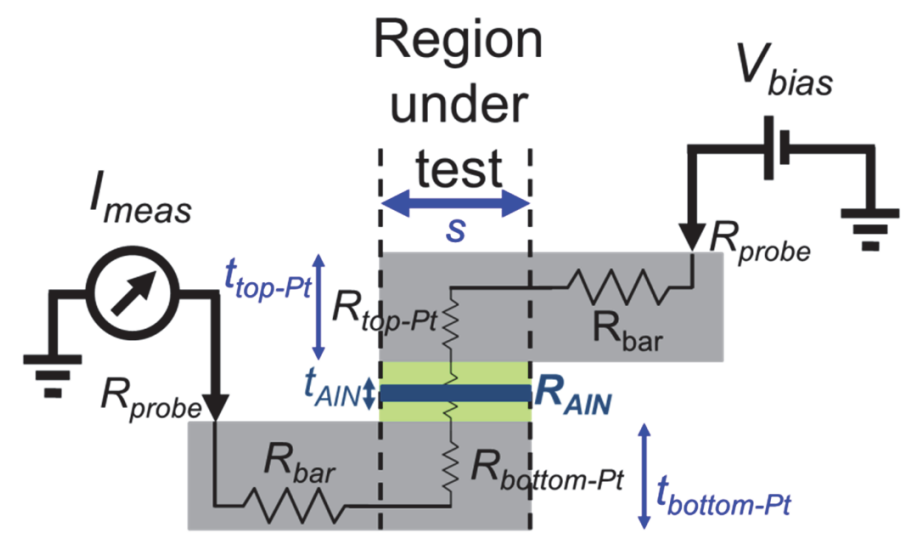

Fig. A 7.3 Equivalent electrical circuit of the test structure with a coalesced AlN layer of resistance $R_{\text {AlN }}\left(\right.$ expressed as $\left.\rho_{\text {AIN }} t_{\text {AlN }} / s^{2}\right)$.

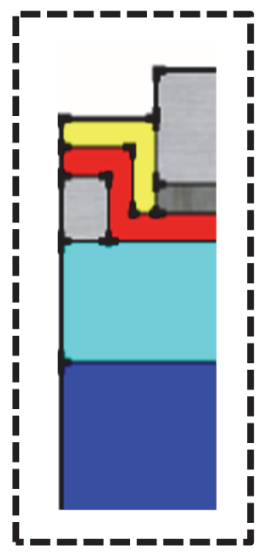

Region of TEM

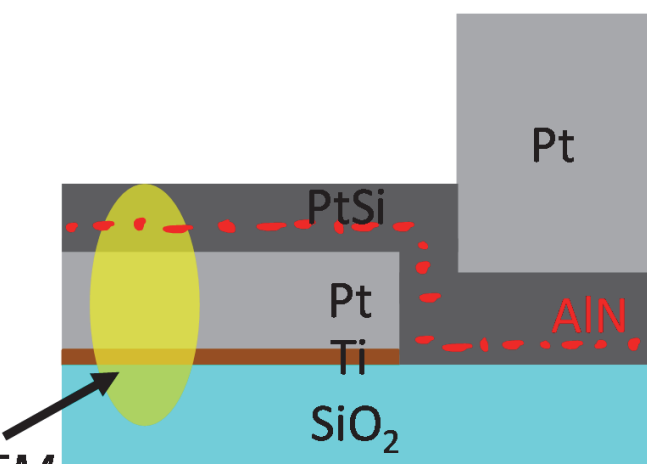

Si

Fig. A 7.4 Cartoon showing the region in the test-structure where the TEM imaging was done (i.e., at the top-left corner of Fig. 7.4 a; its zoom-in is provided within the dashed box.) Instead of a continuous layer, the AIN now exists as clusters (shown in red). 


\section{Conclusions and future recommendations}




\subsection{Conclusions}

The thesis addressed the ALD of polycrystalline AlN and GaN layers from the industrially relevant metalorganic precursors - trimethylaluminium (TMA) and trimethylgallium (TMG), respectively, and ammonia $\left(\mathrm{NH}_{3}\right)$ using various ALD techniques. The work may be divided into two broad sections: (i) radical-enhanced ALD with either hot wire $(\mathrm{HW})$ or plasma for generating radicals, and (ii) radical-free purely thermal ALD.

The thesis started with the exploration of PEALD of these layers (Chapter 2). The deposition conditions, the ALD window, and several material properties including the composition, optical constants, chemical bonding, stoichiometry, morphology and crystallinity were analysed with a variety of in-situ and ex-situ techniques. The polycrystallinity was optimized in terms of the preferential growth of wurtzitic (002) planes, using a variety of means. Among them, the $\mathrm{NH}_{3}$ partial pressure had a major effect, consequently influencing the optical properties of the layers. Optical emission spectroscopy was used to characterize the plasma - detailed in Appendix 2.4. Increased concentrations of atomic- $\mathrm{H}$ and Ar-containing (excited) species were ascribed to the degraded crystallinity at the low partial pressures.

Continuing with radical-enhanced ALD, the novel plasma-free HWALD was pursued in Chapter 3. The dissociation of $\mathrm{H}_{2}$ and $\mathrm{NH}_{3}$ were first studied to gain an insight into the $\mathrm{HW}$ action. The etching of tellurium (Te) films by atomic $\mathrm{H}$ and the nitridation of $\mathrm{Si}$ by $\mathrm{NH}_{\mathrm{x}}(x=0-2)$ radicals were used to investigate the delivery of such radicals from the HW to the substrate. Both in line-of-sight (L-O-S) and outside the L-O-S (with the substrate) positions of the $\mathrm{HW}$ were investigated. The outside position resulted in $\mathrm{AlO}_{\mathrm{x}}$ layers instead of AlN, and Ga droplets instead of GaN. Placing the HW in the L-O-S resulted in $\mathrm{AlO}_{\mathrm{x}} \mathrm{N}_{\mathrm{y}}$ and $\mathrm{GaO}_{\mathrm{x}} \mathrm{N}_{\mathrm{y}}$ layers, respectively. These findings were attributed to the significantly higher reactivity of atomic- $\mathrm{H}$ compared to $\mathrm{NH}_{\mathrm{x}}$ radicals. Another reason could have been the depletion of the $\mathrm{NH}_{\mathrm{x}}$ radicals during the $90^{\circ}$ turn. The higher reactivity of atomic- $\mathrm{H}$ potentially caused the de-methylation of the metalorganic precursors (instead of an efficient nitridation), followed by the oxidation of the de-methylated surface by residual oxidants.

Chapters $4-6$ covered the various aspects of thermal ALD from TMG and $\mathrm{NH}_{3}$, utilizing the hypothesized existence of the TMG: $\mathrm{NH}_{3}$ surface adduct. In Chapter 4, the preparation, characterization and optical properties of composite (the so-called "GaCN") layers were addressed. The layers consisted of nanoscale-size inclusions of GaN, C and Ga. The carbon content had a major influence on the optical bandgap, therefore allowing to prepare high refractive index and low optical bandgap layers by adding only a few-atomic-\% of $\mathrm{sp}^{2}$-hybridized clusters of carbon. A wide range of $\mathrm{GaCN}$ compositions was obtained by varying the deposition temperature and the $\mathrm{NH}_{3}$ partial pressure. The hypothesized 
occurrence of the surface adduct in parallel to the known TMG dissociation mechanism formed the ALD model, explaining the $\mathrm{GaCN}$ composition and growth per cycle (GPC).

The surface-adduct hypothesis was extended to successfully realize the low temperature thermal ALD of GaN films, as presented in Chapter 5. The ALD presumably occurred through (i) the formation of the adduct upon the adsorption of $\mathrm{NH}_{3}$ to a chemisorbed TMG surface site, and (ii) the conversion into of the adduct into a $\mathrm{Ga}-\mathrm{NH}_{2}-\mathrm{Ga}$ linkage signifying a $\mathrm{GaN}$ unit. The hypothesis was supported by similar surface reactions reported in the ALD of AlN layers, as well as gas-phase reactions in GaN MOCVD. The several experimental results, especially the strong dependence of GPC on the $\mathrm{NH}_{3}$ partial pressure which is otherwise atypical of $A L D$, supported the hypothesis. The findings were also in agreement with the simulated kinetics of steps (i) and (ii). The material characterization revealed a polycrystalline wurtzitic GaN layer composed of sub-10 nm grains, slightly Ga rich. The latter was ascribed to the partial dissociation of TMG, preventing the normal occurrence of steps (i) and (ii). Minimizing the dissociation by shortening the cycle time enabled close-to-stoichiometric layers.

The prospect of area selective (AS) ALD utilizing the presence or absence of $-\mathrm{NH}_{2}$ surface- terminations was explored in Chapter 6 at both low pressure (LPALD) and high pressure (HPALD) regimes. In LPALD, such terminations were offered by an in-situ deposited AlN buffer layer and by AlN (sub-)monolayers deposited within super-cycles. In both cases, after an initial TMG chemisorption on the $-\mathrm{NH}_{2}$ terminations, the subsequent $\mathrm{GaN}$ formation was hardly facilitated due to the low pressure. In HPALD, in-situ deposited (and presumably $-\mathrm{NH}_{2}$ terminated) AlN and GaN seed layers readily caused GaN growth; i.e., without a long incubation period. The opposite was observed on $\mathrm{Si}$ and $\mathrm{SiO}_{2}$ substrates lacking the $-\mathrm{NH}_{2}$ terminations. Utilizing the inherently different incubation times on the two classes of surfaces, the ASALD of GaN was performed on patterned $\mathrm{AlN} / \mathrm{SiO}_{2}$ and patterned $\mathrm{Si}_{3} \mathrm{~N}_{4} / \mathrm{SiO}_{2} / \mathrm{Si}$ substrates. In both cases, ex-situ patterning affected the original $-\mathrm{NH}_{2}$ terminations on AlN, causing an almost equal layer thickness on all surfaces. Still, a longer incubation time on $\mathrm{Si}_{3} \mathrm{~N}_{4}$ might potentially lead to ASALD.

The important aspect of the layer coalescence (i.e., its closure thickness making the layer continuous - not necessary to occur at the initial ALD stages) was addressed in Chapter 7. Thermal AlN on PtSi served as an example of substrate-inhibited ALD. Using MIM electrical test structures with AIN films of various thicknesses sandwiched between two PtSi electrodes, the closure thickness was identified from a drastic reduction in current levels and change of the conduction from ohmic to Poole-Frenkel type. The AlN became continuous after reaching $\sim 10 \mathrm{~nm}$ in thickness, being in agreement with the in-situ SE monitored GPC evolution. The pre-coalesced state of AlN at sub-10 nm thicknesses was confirmed by HRTEM analysis. 


\subsection{Future recommendations}

The broad scope of the thesis provides many opportunities for additional research. These will be addressed chapter-by-chapter.

Chapter - 2: In the PEALD of AlN, one of the experiments consisted of annealing (without vacuum break) an in-situ deposited AlN seed layer before proceeding with the remaining AIN ALD (Section 2.2.4.3). It is interesting to investigate the effect of intermittent annealing steps during ALD. Will this enable the growth of epitaxial layers? Investigating such may be important in view of future electronic devices. Besides, the effect of $\mathrm{NH}_{3}-\mathrm{Ar}$ plasma composition on the (Al)GaN crystallinity was studied by OES (Section 2.3.2 and Appendix 2.4). The relative abundance of atomic- $\mathrm{H}$ and (excited) Ar over the $\mathrm{N}$-containing species at low $\mathrm{NH}_{3}$ partial pressures resulted in inefficient nitridation of the films and, presumably, enhanced de-methylation of chemisorbed-TMA and TMG species. This was proposed to cause the degraded crystallinity. The proposition may be tested by OES in a wide range of different plasma conditions. How do the concentrations change with e.g. dissipated power and how this affects the material properties? Further, experiments with $\mathrm{N}_{2}-\mathrm{H}_{2}$ plasma (gives a large degree of freedom for changing the composition - from pure $\mathrm{N}_{2}$ to pure $\mathrm{H}_{2}$ ) instead of $\mathrm{NH}_{3}$ plasma may also be pursued for the same reason.

Chapter - 3: The HW experiments re-iterated the above observation that due to the proposed high reactivity of atomic- $\mathrm{H}$ compared to $\mathrm{NH}_{\mathrm{x}}$ radicals, de-methylation of chemisorbed-TMA and TMG species readily occurs followed by their oxidation at the base pressure of $10^{-7}$ mbar. This results in $\mathrm{AlO}_{\mathrm{x}} \mathrm{N}_{\mathrm{y}}$ and $\mathrm{GaO}_{\mathrm{x}} \mathrm{N}_{\mathrm{y}}$ films instead of pure nitrides (Sections 3.3.2 and 3.4.2). In order to grow the pure nitrides, reducing the base pressure further below $10^{-7} \mathrm{mbar}$ is not practical. Therefore it is essential to significantly increase the $\mathrm{NH}_{\mathrm{x}} /$ at-H ratio in the gas flux. Two approaches may enable such. In the first, since the only $\mathrm{N}$-containing precursor studied in this work was $\mathrm{NH}_{3}$ and changing the filament temperature was unable to achieve the above, another precursor such as $\mathrm{N}_{2}-\mathrm{H}_{2}$ mixture with tunable $\mathrm{N}_{2}$ and $\mathrm{H}_{2}$-contents can be experimented with. In the second, using a $\mathrm{HW}$ stem made of a CMOS-compatible material with high recombination probability of at- $\mathrm{H}$ and O-containing radicals can be investigated.

Chapter - 4: The ability to manipulate the optical properties of $\mathrm{GaCN}$ by varying the $\mathrm{C}$ content may be further explored for specific optical devices. An example of such a device, the combined LED-DBR, is conceptualized in Appendix 4. As suggested there, the LED is encased in a cavity of a GaCN/AlN-based DBR. GaCN, due to its high refractive index, is theoretically shown to outperform GaN as DBR material. The GaCN/AlN DBR has a higher reflectivity than the GaN/AIN DBR at near infrared (NIR) wavelengths. In combination with LED, the DBR not only performs the primary task of allowing to engineer the emission 
wavelength (which can be achieved by $\mathrm{GaCN}$ layers of different compositions), but also improves the spatial spread of the emitted light.

Chapter - 5: This chapter introduced the technique to deposit GaN layers by purely thermal ALD, proposing the TMG: $\mathrm{NH}_{3}$ surface adduct model. Several things remain to be verified in the future. Most importantly, the existence of the surface adduct and its conversion into the $\mathrm{Ga}-\mathrm{NH}_{2}-\mathrm{Ga}$ linkages must be verified using appropriate in-situ surface analysis techniques such as FTIR or XPS. Secondly, it was discussed (Section 5.3.2) that although the initial chemisorption of $\mathrm{TMG}$ on $\mathrm{Si}$ and $\mathrm{SiO}_{2}$ at the deposition temperature of $400{ }^{\circ} \mathrm{C}$ is perhaps not favoured due to the partial dissociation of TMG, the substrates accommodate the necessary $-\mathrm{CH}_{3}$ coverage after $250-300$ ALD cycles to stabilize the GPC. This assumption should also be verified using the in-situ techniques. Finally, the depositions had been performed in a small volume 'experimental' ALD reactor. The pure thermal approach should be tried in a bigger, preferably batch-scale, reactor. Can the adduct-assisted pathway still be realized in a larger-volume commercial reactor?

Chapter - 6: This chapter extended the concept of the TMG: $\mathrm{NH}_{3}$ surface adduct towards achieving GaN ASALD. The concept of ASALD relied on the difference of incubation time on AlN surface (almost no incubation) and on $\mathrm{SiO}_{2}, \mathrm{Si}_{3} \mathrm{~N}_{4}$ and $\mathrm{Si}$ substrates (large incubation). The experiments however showed that standard patterning (i.e., lithography and etching) of the AlN layer affected its $-\mathrm{NH}_{2}$ terminated surface (Section 6.5). Future research should be done towards re-storing the required surface terminations, shortening the incubation time on as-patterned AlN. The still observed large difference in the incubation times between the patterned AlN and $\mathrm{Si}_{3} \mathrm{~N}_{4}$ surfaces (Fig. 6.8) would need an additional investigation to enable ASALD on an $\mathrm{AlN} / \mathrm{Si}_{3} \mathrm{~N}_{4}$ substrate.

Chapter - 7: In this chapter, AlN films deposited by thermal ALD were tested for their coalescence/continuity at the initial ALD stages. Using MIM test-structures and in-situ SE, the coalescence thickness was determined as $\sim 10 \mathrm{~nm}$. For enabling the MIM stack, the film was deposited on a PtSi layer. However, other substrates such as $\mathrm{Si}, \mathrm{SiO}_{2}$, in-situ deposited AlN and GaN seed-layers, metals, etc. can be further explored as relevant for making future electronic devices. For the latter, studying the initial growth of thermal GaN layers is certainly of interest.

Last but not the least: the polycrystalline AlN \& GaN layers prepared in this work must be characterized in terms of their electrical performance, as being pursued in a sister project. 


\section{Summary}

In order to continue the ever-increasing miniaturization trend of Silicon ( $\mathrm{Si}$ )-based microelectronic components (such as microprocessor, memories and logic that are used for high-performance digital computing), in an era when we have almost fully-exploited the physical capabilities of $\mathrm{Si}$, various other semiconductors that can potentially complement $\mathrm{Si}$ are being at present intensively researched.

Gallium nitride $(\mathrm{GaN})$ and aluminium nitride (AlN) are so-called compound semiconductors which, in their monocrystalline form, have certain material properties superior to $\mathrm{Si}$; e.g., direct and wider bandgap, high electron mobility and high breakdown field. However, the application of these semiconductors has been restricted to the optoelectronics and communication domains, such as light emitting diodes (LED) and high electron mobility transistors (HEMT). This is because, the progress of Si-based microelectronics (and associated process technology) has been propelled by the easy availability / ease-of-manufacturing of monocrystalline $\mathrm{Si}$ substrates, and the superb insulating property and interface qualities of silicon dioxide. These properties are exploited to fabricate transistors: the building block of digital electronics. In comparison, the substrates suitable for $(\mathrm{Al}) \mathrm{GaN}$-based electronics, such as bulk $\mathrm{Al}(\mathrm{GaN})$ crystals, $\mathrm{SiC}$ and Sapphire, have been prohibitively expensive to allow their mass-scale production, and also posed several challenges to achieve good material and electronic properties of the over-grown (Al)GaN layers. Therefore, whereas the Si-based microelectronic devices have become ubiquitous in every-day applications (e.g., smart-phones, internet-of-things, etc.), their (Al)GaN-counterparts have been limited mainly to opto-electronic applications, and the two technologies have barely crossed paths.

Combining the power of the mature Si-based process technology with the superior material properties of (Al)GaN semiconductors on one platform potentially enables future microelectronic devices in accordance with the 'More-than-Moore' philosophy. In parallel, exploring the polycrystalline and thin film (i.e., thicknesses below a micron) versions of (Al)GaN is also attractive, since that helps to diversify their growth requirements and process conditions. For example, polycrystalline-(Al)GaN layers can be deposited on a large range of substrates, beyond $\mathrm{Si}$ (such as glass and plastic) and by several physical and chemical deposition techniques. Further, they can be deposited under a wide range of temperatures and using a variety of precursors. Finally, polycrystalline-(Al)GaN layers also enable the addition of foreign elements for tuning their material and electronic properties. All these abovementioned features allow for the realization of a wide array of micro- and opto-electronic devices, beyond the traditional LED and HEMT applications. 
Atomic layer deposition (ALD) is a highly relevant technique for poly-(Al)GaN layers in view of their usage in current and future device applications. This is because of the unprecedented level of control over the layer thicknesses (almost reaching atomic level precision) that the technique offers. In addition, ALD ensures a high-degree of spatial uniformity of thickness and material properties, useful in the currently used large-sized (e.g., $300 \mathrm{~mm}$ in diameter) wafers, as well as offers superb conformality, which is useful for fabricating three-dimensional devices with high-aspect-ratios.

On this premise, this thesis addressed the ALD of polycrystalline (Al)GaN thin films on Si and Si-compatible substrates. A variety of activation techniques, ranging from thermal, plasma to the novel hot-wire ALD, were explored. Reports of ALD of (Al)GaN is appearing only recently in the literature, which suggests that the field is gaining prominence. Keeping in mind the possible utilization of the research results in the industry, standard industrial precursors were used. Several novel results were obtained which are recapitulated below.

The research started with developing recipes for $(\mathrm{Al}) \mathrm{GaN}$ layers with plasmaenhanced ALD (Chapter 2). The changes to the layer polycrystallinity were explored by varying the plasma composition, substrates, etc. Diagnosis of the plasma provided important insights into the underlying causes behind changes to the polycrystallinity and the associated layer optical properties. The deposition of $(\mathrm{Al}) \mathrm{GaN}$ was subsequently explored by the plasma-free radical-assisted hot-wire ALD technique (Chapter 3). The experiments provided critical insights into the generation and delivery of various radicals, obtained from the dissociation of the nitrogen-containing precursor, from the hot-wire to the substrate. Moreover, the results showed the relative influence of these radicals in the layer growth mechanism, and showed their changing effects with changes to the reactor geometry and deposition conditions. Such insights paved the way towards realising a novel purely-thermal, radical-free ALD process for GaN and GaCN layers (Chapter 4 and 5). The composition of such layers were charted under a wide range of deposition temperatures and reactor pressures. It was shown that, carbon, obtained from the dissociation of the Ga-containing precursor, had a profound influence on the optical properties of the layers. A conceptual optical device was proposed based on this observation. Moreover, the atypical observation of the pressure-dependent thermal $A L D$ of $G a N$ was reported, along with a possible explanation behind the growth mechanism. This also provided the means to selectively deposit GaN layers (Chapter 6), which is highly relevant field of industrial ALD research. Finally, the aspect of coalescence (or closure) of thin sub-10 nm AlN films, formed during the initial stages of ALD, were investigated using electrical and optical techniques (Chapter 7). The pre-coalesced cluster-like state of AlN was also shown. In conclusion, the results obtained from this thesis, and the possible implementation of the suggested future work, are expected to advance the state-of-the-art in $(\mathrm{Al}) \mathrm{GaN}$ and ALD research. 


\section{Samenvatting}

Ten einde de steeds toenemende miniaturisatie trend van silicium ( $\mathrm{Si}$ ) gebaseerde micro-elektronische componenten (zoals microprocessoren, geheugen chips en logica voor high-performance processoren) mogelijk te maken in een tijdperk waarin bijna alle mogelijkheden van de fysieke limieten van $\mathrm{Si}$ al worden uitgebuit, worden diverse andere halfgeleider materialen, die mogelijkerwijs $\mathrm{Si}$ kunnen complementeren uitgebreid onderzocht.

Gallium Nitride (GaN) en Aluminium Nitride (AlN) zijn zogenaamde samengestelde halfgeleiders die, in hun monokristallijne vorm, bepaalde materialen eigenschappen bezitten, die beter zijn dan die van $\mathrm{Si}$; bijvoorbeeld, een directe en grotere band afstand, hoge elektronen mobiliteit en een hoog doorslag veld. De toepassing van deze materialen echter, is tot nu toe beperkt tot de opto-electronische en communicatie domeinen, zoals bijv. light emitting diodes (LEDs) en hoge electron mobiliteit transistoren (HEMTs). Dit komt vooral door de vooruitgang van de Si-gebaseerde micro-elektronica (en de daarmee geassocieerde proces technologie) die gebaseerd is op de beschikbaarheid en de relatief eenvoudige fabricage van monokristallijne Si substraten, de superieure isolerende en grensvlak eigenschappen van siliciumdioxide. Deze eigenschappen worden uitgebuit in de fabricage van transistoren, de bouwstenen van digitale elektronica. Ter vergelijking: de substraten die geschikt zijn voor (Al)GaN gebaseerde elektronica, zoals (Al)GaN bulk kristallen, Silicium Carbide of saffier, zijn te duur voor massa productie, en het is ook een uitdaging om goede materiaal en elektronische eigenschappen te verkrijgen van de $\mathrm{Al}(\mathrm{Ga}) \mathrm{N}$ lagen die er op gegroeid worden. Daarom zijn de (Al)GaN micro-elektronische devices grotendeels beperkt tot opto-electronische toepassingen, dit in tegenstelling tot de alom aanwezige Si gebaseerde micro-elektronische devices(zoals smart phones, internet-of-things, etc.), en zijn de twee technologieën grotendeels gescheiden gebleven.

De combinatie van de mogelijkheden van volwassen Si-gebaseerde proces technologie met de superieure materiaal eigenschappen van $(\mathrm{Al}) \mathrm{GaN}$ op één platform, maakt het potentieel mogelijk om toekomstige micro-elektronische devices te maken in overeenstemming met de 'More than Moore' filosofie. Tegelijkertijd is het onderzoek aan polykristallijne en dunne films (met een dikte minder dan een paar micron) van $\mathrm{Al}(\mathrm{Ga}) \mathrm{N}$ ook aantrekkelijk, omdat het helpt de groei omstandigheden en proces condities uit te breiden. Bijvoorbeeld, polykristallijne $\mathrm{Al}(\mathrm{Ga}) \mathrm{N}$ lagen kunnen gedeponeerd worden op een groot aantal substraten, naast silicium ook op bijvoorbeeld glas en plastic, gebruikmakend van verschillende fysische en chemische depositietechnieken. Bovendien kunnen ze aangebracht worden in een breed temperatuur gebied, gebruikmaken van een verscheidenheid aan precursors. Ten slotte, polykristallijne $\mathrm{Al}(\mathrm{Ga}) \mathrm{N}$ lagen maken het ook mogelijk om vreemde elementen in te bouwen, en daarmee de materiaal en elektronische eigenschappen af te 
stemmen. De boven vermelde eigenschappen maken de realisatie mogelijk van een breed scala aan micro- en opto-electronische devices, naast de traditionele LED en HEMT toepassingen.

Atomaire Laag Depositie (ALD) is een zeer geschikte techniek voor poly- $\mathrm{Al}(\mathrm{Ga}) \mathrm{N}$ lagen gezien het gebruik en in huidige en toekomstige device toepassingen. Dit komt door de zeer hoge controleerbaarheid van de laagdiktes (bijna een atoom laag nauwkeurigheid) van deze techniek. Bovendien heeft ALD een hoge mate van ruimtelijke uniformiteit van de dikte en materiaal eigenschappen, wat zeer bruikbaar is in de huidige grote (bijvoorbeeld $300 \mathrm{~mm}$ diameter) plakken; bovendien biedt het uitermate goede stapbedekking,wat nuttig voor de fabricage van driedimensionale structuren met hoge aspect verhoudingen.

Uitgaand van deze premisse behandelt dit proefschrift de ALD van dunne, polykristallijne $\mathrm{Al}(\mathrm{Ga}) \mathrm{N}$ films op Silicium en Silicium compatibele substraten. Een verscheidenheid van activeringstechnieken, variërend van thermische, plasma, tot het nieuwe hot-wire ALD werden bestudeerd. Publicaties van ALD van Al(Ga)N verschijnen pas sinds kort in de literatuur, wat suggereert dat het onderzoeksveld aan belang toeneemt. De mogelijke toepassing van de onderzoeksresultaten in de industrie werden continu in ogenschouw genomen, en daarom werden standaard industriële precursors gebruikt. Verscheidene nieuwe resultaten werden verkregen. Een korte samenvatting wordt hieronder gegeven.

Het onderzoek begon met de ontwikkeling van recepten voor $\mathrm{Al}(\mathrm{Ga}) \mathrm{N}$ lagen met plasma-enhanced ALD (hoofdstuk 2). De veranderingen in de polykristalliniteit ten gevolge van variatie in de plasma condities, substraten, etc., werden bestudeerd. Diagnose van het plasma leverde belangrijke inzichten in de onderliggende oorzaken van de veranderingen van de polykristalliniteit en de bijbehorende optische eigenschappen van de lagen. Vervolgens is de depositie van $\mathrm{Al}(\mathrm{Ga}) \mathrm{N}$ onderzocht met plasmaloze 'radical-assisted' hot-wire ALD techniek (hoofdstuk 3). De experimenten leverden belangrijke inzichten in de generatie en de levering van de diverse radicalen, verkregen door de dissociatie van de stikstof bevattende precursor, van de hot-wire bron naar het substraat. Bovendien toonden de resultaten de de invloed van deze radicalen op het groei mechanisme van de laag, en de invloed van de veranderingen in de reactor geometrie en depositie omstandigheden. Deze inzichten maken het mogelijk om een nieuw geheel thermisch, vrij van radicalen, ALD proces voor GaN en $\mathrm{GaCN}$ te ontwikkelen (hoodstuk 4 en 5). De samenstelling van deze lagen werd in kaart gebracht over een groot gebied van depositie temperaturen en reactor drukken. Aangetoond werd, dat koolstof, dat ontstond uit de dissociatie van de Ga bevattende precursor, een grote invloed had op de optische eigenschappen van de lagen. Het concept van een optisch device gebaseerd op deze waarneming wordt besproken. Bovendien wordt de atypische waarneming van de drukafhankelijkheid van de ALD van GaN gerapporteerd, samen met een mogelijke verklaring van het groei mechanisme. Dit maakt het ook mogelijk om selectief GaN lagen te 
deponeren (hoofdstuk 6), wat interessant is voor industriële ALD research. Ten slotte, is coalescentie (of sluiting) van dunne AlN lagen van minder dan $10 \mathrm{~nm}$ onderzocht gebruik makend van elektrische en optische technieken (hoofdstuk 7). Er werden ook AlN lagen met clusters die nog niet gesloten bestudeerd.

Samenvattend, de verwachting is dat de resultaten van dit proefschrift, en de mogelijke implementatie van het gesuggereerde toekomstige werk de state-of-the-art van $\mathbf{A l}(\mathbf{G a}) \mathbf{N}$ vooruit helpen. 


\section{Scientific contributions from PhD}

\section{Journals}

Banerjee, S.; Aarnink, A. A. I.; Gravesteijn, D. J.; Kovalgin. A. Y., Thermal Atomic Layer Deposition of Polycrystalline Gallium Nitride. The Journal of Physical Chemistry C (under review).

Banerjee, S.; Onnink, A. J.; Dutta, S.; Aarnink, A. A. I.; Gravesteijn, D. J.; Kovalgin, A. Y., Composite GaN-C-Ga ("GaCN") Layers with Tunable Refractive Index. The Journal of Physical Chemistry C 2018, 122(51), 29567-29576.

(DOI: 10.1021/acs.jpcc.8b09142)

Gupta, G.; Banerjee, S.; Dutta, S.; Aarnink, A. A. I.; Schmitz, J.; Kovalgin, A. Y.; Hueting, R. J. E., Charge Carrier Transport and Electroluminescence in Atomic Layer Deposited Poly-GaN/C-Si Heterojunction Diodes. Journal of Applied Physics 2018, 124(8), 084503.

(DOI: 10.1063/1.5041089)

Banerjee, S.; Kovalgin, A. Y., A Comparative Study of Low-Temperature III-V Nitrides ALD in Thermal and Radical-Enhanced Modes. ECS Transactions 2018, 86(6), 21-29.

(DOI: $10.1149 / 08606.0021$ ecst)

Gupta, G.; Dutta, S.; Banerjee, S.; Hueting, R. J. E., Minority Carrier Injection in High-Barrier Si-Schottky Diodes. IEEE Transactions on Electron Devices 2018, 65(4), 1276-1282.

(DOI: 10.1109/TED.2018.2807926)

Kovalgin, A. Y.; Yang, M.; Banerjee, S.; Apaydin, R. O.; Aarnink, A. A. I.; Kinge, S.;

Wolters, R. A. M., Hot-Wire Assisted ALD: A Study Powered by In Situ Spectroscopic Ellipsometry. Advanced Materials Interfaces 2017, 4(18), 1700058.

(DOI: 10.1002/admi.201700058)

Banerjee, S.; Aarnink, A. A. I.; van de Kruijs, R.; Kovalgin, A. Y.; Schmitz, J., PEALD AlN: Controlling Growth and Film Crystallinity. physica status solidi (c) 2015, 12(7), 1036-1042.

(DOI: 10.1002/pssc.201510039)

\section{Conferences}

Banerjee, S.; Aarnink, A. A. I.; Gravesteijn, D. J.; Kovalgin, A. Y., Inherent Substrate-selective Atomic Layer Deposition of Polycrystalline Gallium Nitride.

(oral presentation at EuroCVD 22-Baltic ALD 16 conference, Luxembourg City, Luxembourg, 2019).

Banerjee, S.; Dutta, S; Aarnink, A. A. I.; Schmitz, J.; Gravesteijn, D. J.; Kovalgin, A. Y., Purely Thermal Deposition of Polycrystalline Gallium Nitride Films at $400{ }^{\circ} \mathrm{C}$.

(oral presentation at $A L D 2018$ conference, Incheon, Republic of Korea, 2018).

Banerjee, S.; Apaydin, R. O.; Aarnink, A. A. I.; Schmitz, J.; Gravesteijn, D. J.; de Jong, M. P.; Kovalgin A. Y., Hot Wire Technique as a Radical Source for Atomic Layer Deposition of Metal \& Metal Nitride films.

(oral presentation at EuroCVD 21-Baltic ALD 15 conference, Linköping, Sweden, 2017). 
Banerjee, S.; van der Velde, R.; Yang, M.; Schmitz, J.; Kovalgin, A. Y., Electrical Test Structures for Verifying Continuity of Ultra-Thin Insulating and Conducting Films.

(oral presentation \& proceeding at ICMTS 2017 conference, Grenoble, France, 2017).

(DOI: 10.1109/ICMTS.2017.7954258)

Banerjee, S.; Aarnink, A. A. I.; Kip, G. A.; Gravesteijn, D. J.; Schmitz, J.; Kovalgin, A. Y., Deposition of Thin Layers Containing $\mathrm{Ga}, \mathrm{C}$ and $\mathrm{N}$ by Sequential Pulses of Trimethylgallium and Ammonia. (poster presentation at $A L D 2016$ conference, Dublin, Ireland, 2016).

Banerjee, S.; Aarnink, A. A. I.; van de Kruijs, R.; Kovalgin, A. Y.; Schmitz, J., PEALD AIN: Controlling Growth and Film Crystallinity.

(oral presentation \& proceeding at EuroCVD 20 conference, Sempach, Switzerland, 2015).

(DOI: 10.1002/pssc.201510039)

\section{$\underline{\text { Patent }}$}

Banerjee, S.; Aarnink, A. A. I.; Kovalgin, A. Y., METHOD OF FORMING A DEVICE STRUCTURE USING SELECTIVE DEPOSITION OF GALLIUM NITRIDE AND SYSTEM FOR SAME. Applicant: ASM IP Holding B. V. (The Netherlands), Filed: November 2019, Status: Provisional. 


\section{Scientific contributions before PhD}

\section{Journals}

Vollebregt, S.; Banerjee, S.; Tichelaar, F. D.; Ishihara, R., The Growth of Carbon Nanotubes on Electrically Conductive ZrN Support Layers for Through-Silicon Vias. Microelectronic Engineering 2016, 156, 126-130. (DOI: 10.1016/j.mee.2016.01.034)

Vollebregt, S.; Banerjee, S.; Chiaramonti, A. N.; Tichelaar, F. D.; Beenakker, K.; Ishihara, R., Dominant Thermal Boundary Resistance in Multi-Walled Carbon Nanotube Bundles Fabricated at Low Temperature. Journal of Applied Physics 2014, 116(2), 023514. (DOI: 10.1063/1.4889820)

Vollebregt, S.; Banerjee, S.; Beenakker, K.; Ishihara, R., Size-Dependent Effects on the Temperature Coefficient of Resistance of Carbon Nanotube Vias. IEEE Transactions on Electron Devices 2013, 60(12), 4085-4089. (DOI: 10.1109/TED.2013.2287640)

Vollebregt, S.; Banerjee, S.; Beenakker, K.; Ishihara, R., Thermal Conductivity of Low Temperature Grown Vertical Carbon Nanotube Bundles Measured Using the Three- $\Omega$ Method. Applied Physics Letters 2013, 102(19), 191909. (DOI: 10.1063/1.4805069)

Satapathy, N.; Pandey, D.; Banerjee, S.; Ramachandran, H., Optical Phase Noise Engineering Via Acousto-Optic Interaction and Its Interferometric Applications. Journal of the Optical Society of America A 2013, 30(5), 910-915. (DOI: 10.1364/JOSAA.30.000910)

\section{Conference proceedings}

Vollebregt, S.; Banerjee, S.; Beenakker, C.; Ishihara, R. Ultralong Vertically Aligned Carbon Nanotubes Grown on Conductive ZrN Layers, Proc. of Int'l. Conference on Diamond and Carbon Materials, 2013, pp O. 044-1-2.

Vollebregt, S.; Banerjee, S.; Tichelaar, F. D.; Ishihara, R. Carbon Nanotubes TSV Grown on an Electrically Conductive ZrN Support Layer, Interconnect Technology Conference and 2015 IEEE Materials for Advanced Metallization Conference (IITC/MAM), 2015 IEEE International, IEEE: 2015, 281-284. (DOI: 10.1109/IITC-MAM.2015.7325667)

\section{Masters thesis}

Banerjee, S., Super-Growth of CNTs Based on ZrN for TSV Application. 2014. TU Delft, The Netherlands. (http://resolver.tudelft.nl/uuid:1fa0028e-80c5-447d-b28e-6cd77730f133) 


\section{Acknowledgements}

First and foremost, sincere thanks to my project leader, my daily supervisor and Co-promotor, dr. Alexey Y. Kovalgin, who, along with dr. ir Ray J. E. Hueting and prof. dr. Jurriaan Schmitz, initiated the project "Towards polycrystalline GaN/AlGaN devices in silicon technology" in 2014. They selected me as the $\mathrm{PhD}$ candidate to work on the growth and characterization aspects of poly-(Al)GaN thin film materials, providing the opportunity to learn the technique of Atomic Layer Deposition (ALD) in the state-of-the-art cleanroom of the MESA+ Institute for Nanotechnology at the University of Twente. My sincere gratitude also to prof. dr. Dirk J. Gravesteijn for willing to be the Promotor of my PhD. I shall write about each of you later, but additional thanks for initiating the joint $\mathrm{PhD}$ research projects on poly-GaN (consisting of myself and another $\mathrm{PhD}$ candidate working on the electrical characterization). This set up a collaborative and intensive $\mathrm{GaN}$ research environment in the group, where me and my colleague could discuss several things. I do feel extremely fortunate to be a part of the Group for more than 5 years, getting to work with, and learn form, the industry veterans!

I express my gratitude to the Nederlandse Organisatie voor Wetenschappelijk Onderzoek (NWO) and to the users of the project (NXP Semiconductors, Koninklijke Philips, ASM International, EDAX, TSST and Tyndall National Institute) for providing financial and intellectual support. In the same lines, my sincere thanks and regards to the Graduation Committee, consisting of prof. dr. ir. J. R. van Ommen (TU Delft), prof. dr. ir. W. M. M. Kessels (TU Eindhoven), prof. dr. C. Detavernier (University of Ghent), dr. ir. J. W. Maes (ASM International N. V.), besides prof. dr. ing. A. J. H. M. Rijnders, prof. dr. Dirk J. Gravesteijn, dr. Alexey Y. Kovalgin and dr. ir Ray J. E. Hueting (University of Twente) for their willingness to be in the committee, patience to read the thesis amidst their busy schedules, and also suggesting improvements to the same.

Alexey, I would rather not waste too many words trying to describe how much I'm indebted to you. Our continuous interaction over the last 5 years saw many twists-and-turns. We enjoyed the successes of our research at conferences and in the form of publications. But also, not every time we seemed to be in agreement! According to me, that is acceptable and perhaps useful in science. For instance, although you had a persistent notion that the hotwire-assisted (HWALD) technique would be successful for (Al)GaN layers (using the current precursors) whereas I was sceptical about the same, the insights obtained during those 1.5 years of the numerous trial-and-error (mostly futile) experiments, trying to understand the HW operation, in fact opened up the route towards realizing the potential pressure-dependence of thermal GaN ALD! THANK YOU for patiently teaching me the art of doing research, critically correcting the manuscripts, and showing how to communicate scientific results in a clear and concise way. Similarly, I'm inspired by your qualities of remaining calm, patient and efficient in executing multiple research projects. In the same lines, my fullest gratitude to Dirk and Jurriaan. All three of you spent significant portions of your time teaching me the skill of presenting the research results - through presentations and publications - in a professional, scientific manner. Dirk, thank you for sharing your expertise on $\mathrm{GaN}$ technology which you had acquired in your long career, on many occasions of this 
$\mathrm{PhD}$, for contributing to our joint publications, and of course, for painstakingly reading, correcting and improving the quality of this thesis. One rarely finds such a friendly, spirited and encouraging supervisor as you, under whom I never felt shy or scared to propose ideas, without thinking twice if they could be stupid. Such an open communication is very important in a PhD, I believe. Last but not the least, thanks for agreeing to translate the thesis summary to Dutch! Jurriaan, my genuine gratitude to you for allowing me to continue with the experimental and analyses plans, even after the project budget had been exceeded by quite some margin. For instance, I can remember that afternoon when the turbo-pump went bang! Still, you tolerated and allowed me to continue with depositions, agreeing to install a second turbo immediately. Finally, thanks for allowing me to present at two more conferences, attend a workshop, and use the office, even after the end of the PhD contract.

Thanks to the several other faculty members and the current and former $\mathrm{PhD}$ students of the Integrated Devices and Systems Group (especially the former Semiconductor Components division) from whom I had the opportunity to learn various academic and nonacademic things. I chose not to name them individually for conciseness, but that doesn't undermine their significance in any way. However, it would be utter injustice if some of them go unmentioned! First and foremost, a big THANK YOU to Tom Aarnink, our senior Process Engineer. Without your intensive supervision to the green-horn $\mathrm{PhD}$ students in the cleanroom, our research would have gone nowhere! With your unending patience, you "baby-sit" us throughout our PhD duration, and carefully teach us the intricacies of the ALD reactors. The amount of equipment-time that I received in this group, was, needless-to-say, a tremendous benefit. Whenever I was stuck with any difficulty in the cleanroom, a phone-call was enough to get you into action (once, even after office hours when you rushed from your house), and receive your support. But not only the technical nitty-gritties, I will also miss your warm and social nature, and I shall fondly recollect our long afternoon chats about... everything really. Words won't really suffice my indebtedness to you, Tom. Thank you!

A trusted friend and colleague as Satadal doesn't occur every time. How much I'm grateful to you for re-teaching some important concepts in Material Science and Chemistry, which we had learnt in our undergraduate classes, but which I had efficiently managed to forget. For instance, without your clear explanations, I would neither have learnt what the Langmuir isotherm is, nor how XRD works. It was useful exercise to make sense of those early surface chemistry papers, based on which, we could come up with an explanation of the GaN ALD experiments. Also, I gratefully acknowledge your experimental support for the plasma spectroscopy measurements. I, like you, will always remember the discussions and chats we had on and off work, that crazy road-trip to Swedish Lapland, and really, the numerous fond events here-n-there. You were also the first one to encourage me to learn Dutch through your own example. And to top it off, thanks for agreeing to be my Paranymph too, amidst your Post-Doc schedule. Tihomir, although we are acquainted only for a few months now, I sincerely thank you for enthusiastically willing to be my other Paranymph, amidst your busy Post-Doc schedule. Arnoud, with your background as a Master in Chemistry, you helped to bring in valuable expertise in the group. Your passion for science, though overbearing at times, helped us compose that ten-page-long paper on the novel $\mathrm{GaCN}$ composite material and publish it in a very respectable journal. It was a pleasure to have both Satadal and you as co-authors. Gaurav, you had really been an excellent colleague to work 
with on the poly-GaN project. Your professional background in epi-GaN growth helped me understand the differences between epi- and poly-(Al)GaN materials, and thus I could appreciate the premise of my $\mathrm{PhD}$ better. Our collaboration proved to be successful so far, and I consider myself fortunate to co-author two papers with you. On personal fronts, I cherished the many conversations we had off work. Oguzhan, my sincere gratitude for your support with several HWALD experiments, which contributed to a conference presentation and a journal publication. Mengdi, I congratulate you for becoming a mother, and fondly remember our early days as young $\mathrm{PhD}$ students. Bernhard, thank you for proof-reading the thesis and I congratulate you on further continuing the poly-GaN project in your $\mathrm{PhD}$. Good luck! Finally, my regards to the friendly Remke Assink, our former Group Secretary, for arranging every kind of logistics with care and ease.

To all the personnel of the MESA+ Nanolab: A BIG THANK YOU! Again I would skip naming each of them for brevity, and because of some way or the other, I have taken help from each one of them. However, special acknowledgements go to Gerard Kip and Robert Wijn. Gerard kept himself available literally every time when I had questions regarding XPS, resulting in frequent and long discussions about the measurements and analyses, for which I'm truly grateful. Robert, thank you for being there every time when I had doubts and difficulties in the cleanroom, never hesitating to share your wealth of experience. I'm extremely honoured to have worked with you in the last 5 years. Mark Smithers and Rico Keim, we spent a good number of hours acquiring and interpreting electron microscope images. I thoroughly enjoyed the experience, and just a 'thanks' may not be enough to express my gratitude! Last but not the least, my sincere acknowledgements to Robbert van de Kruijs and Marko Sturm, both assistant professors in the XUV Optics Group of this university, for the several technical discussions that we had, particularly for performing / helping with some of the XRD, XPS and LEIS measurements.

To all the old and new friends and colleagues at Twente and everywhere else. I am still grateful to dr. ir. Sten Vollebregt, my Masters thesis mentor and currently Assistant Professor at TU Delft, for providing the opportunity to work with and publish several papers together. That was surely an important step to getting this $\mathrm{PhD}$ position at Twente. To the many peers I interacted with at the ALD and EuroCVD conferences. To the University of Twente in general, for providing a serene atmosphere that is ideal for contemplating and conducting research. The campus is your jewel and I've been fortunate to live in the middle of it for a long 4.5 years. Here, all my Dutch Matenweg housemates deserve special mention. My warmest regards to you for allowing me, a foreigner, to experience the typical Dutch student-life! You patiently tolerated (two strong character traits of the Dutch) my occasional nonchalance to the rules and organization of the student-house, but still kept the dinner ready for me every single evening while I was away working in the lab. Thanks mates! All of you and also Annemarie, helped me to prepare for the Inburgering. Without passing that and subsequently receiving the permanent residence of the Netherlands, I could have never been stress-free during the final year, and after my PhD contract. In fact, I used the opportunity to attend two more conferences in the $5^{\text {th }}$ year. Thank you!

In this long list, if I've managed to forget a few names, I do apologize for the error. Finally, I would end by thanking Baba and Ma, for lending your relentless strength, support and inspiration to all my activities. To newer adventures then ... 


\section{Epilogue}

Pangloss used now and then to say to Candide:

"There is a concatenation of all events in the best of possible worlds; for, in short, had you not been kicked out of a fine castle for the love of Miss Cunegund; had you not been put into the Inquisition; had you not travelled over America on foot; had you not run the Baron through the body; and had you not lost all your sheep, which you brought from the good country of El Dorado, you would not have been here to eat preserved citrons and pistachio nuts.

"Excellently observed," answered Candide; "but let us cultivate our garden."

CANDIDE, Voltaire, 1759

(C) 1998, Electronic Scholarly Publishing Project 


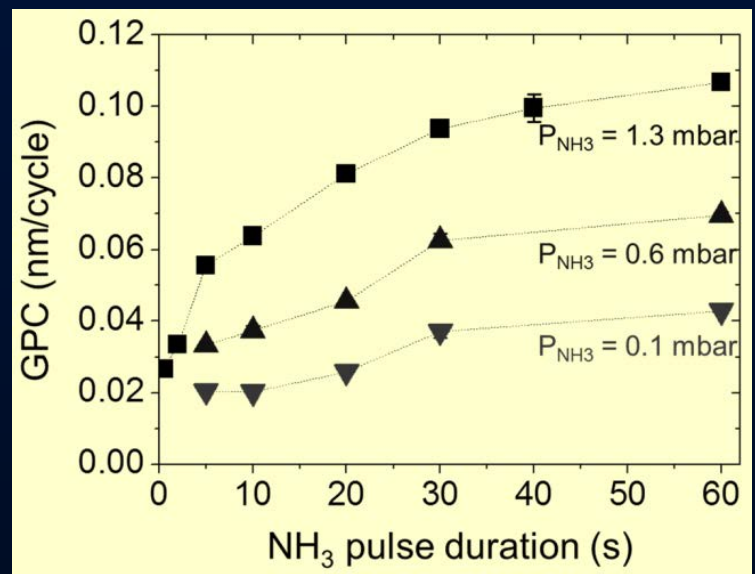

